\title{
Conquer your cravings
}

Citation for published version (APA):

Schyns, G. L. T. (2018). Conquer your cravings: investigating the effects and working mechanisms of food cue exposure in overweight and obese individuals. [Doctoral Thesis, Maastricht University]. Maastricht University. https://doi.org/10.26481/dis.20180209gs

Document status and date:

Published: 01/01/2018

DOI:

10.26481/dis.20180209gs

Document Version:

Publisher's PDF, also known as Version of record

\section{Please check the document version of this publication:}

- A submitted manuscript is the version of the article upon submission and before peer-review. There can be important differences between the submitted version and the official published version of record.

People interested in the research are advised to contact the author for the final version of the publication, or visit the DOI to the publisher's website.

- The final author version and the galley proof are versions of the publication after peer review.

- The final published version features the final layout of the paper including the volume, issue and page numbers.

Link to publication

\footnotetext{
General rights rights.

- You may freely distribute the URL identifying the publication in the public portal. please follow below link for the End User Agreement:

www.umlib.nl/taverne-license

Take down policy

If you believe that this document breaches copyright please contact us at:

repository@maastrichtuniversity.nl

providing details and we will investigate your claim.
}

Copyright and moral rights for the publications made accessible in the public portal are retained by the authors and/or other copyright owners and it is a condition of accessing publications that users recognise and abide by the legal requirements associated with these

- Users may download and print one copy of any publication from the public portal for the purpose of private study or research.

- You may not further distribute the material or use it for any profit-making activity or commercial gain

If the publication is distributed under the terms of Article $25 \mathrm{fa}$ of the Dutch Copyright Act, indicated by the "Taverne" license above, 


\section{CONQUER YOUR CRAVINGS}

Investigating the effects and working mechanisms of food cue exposure in overweight and obese individuals

Ghislaine Schyns 
ISBN: $\quad 978-94-6299-865-0$

Lay-out: Nikki Vermeulen - Ridderprint BV

Printing: Ridderprint BV - www.ridderprint.nl

(C) Ghislaine Schyns, 2018 


\section{CONQUER YOUR CRAVINGS}

Investigating the effects and working mechanisms of food cue exposure in overweight and obese individuals

\section{PROEFSCHRIFT}

ter verkrijging van de graad van doctor aan de Universiteit Maastricht, op gezag van de Rector Magnificus, Prof. dr. Rianne M. Letschert, volgens het besluit van het College van Decanen,

in het openbaar te verdedigen

op vrijdag 9 februari 2018 om 12.00 uur

door

Ghislaine Léonie Thérèse Schyns 


\section{PROMOTOR}

Prof. Dr. Anita Jansen

\section{CO-PROMOTOR}

Dr. Anne Roefs

\section{BEOORDELINGSCOMMISSIE}

Prof. Dr. Johan W. S. Vlaeyen (voorzitter)

Dr. Chantal Nederkoorn

Prof. Dr. Marleen M. Rijkeboer

Prof. Dr. Marcel A. Van den Hout

Dr. Bram Vervliet

The research presented in this dissertation is financed by the Netherlands Organisation for Scientific Research (NWO): Vici Grant 453.10.006, awarded to Anita Jansen. 


\section{TABLE OF CONTENTS}

$\begin{array}{lll}\text { CHAPTER } 1 \quad \text { General Introduction } & 7\end{array}$

CHAPTER 2 Expectancy violation, reduction of food cue reactivity and 19

less eating in the absence of hunger after one food cue exposure session for overweight and obese women

CHAPTER 3 Cue exposure therapy reduces overeating of exposed and non-exposed foods in obese adolescents

CHAPTER 4 Enhancing inhibitory learning to reduce overeating:

Design and rationale of a cue exposure therapy trial in overweight and obese women

CHAPTER 5 Weight loss and binge eating reduction after eight sessions food cue exposure therapy aimed at inhibitory learning in obesity

CHAPTER 6 What works better? Food cue exposure aiming at the habituation of eating desires or food cue exposure aiming at the violation of overeating expectancies?

CHAPTER 7 The power chapter

CHAPTER 8 General discussion

References

Summary

Valorisation addendum

Samenvatting

Dankwoord

Curriculum vitae 



\section{CHAPTER}

\section{GENERAL INTRODUCTION}



"The only way to keep your health is to eat what you don't want, drink what you don't like, and to do what you'd rather not" (Twain, 1897, p. 459).

\section{OBESITY AND FOOD CUE REACTIVITY}

Although Twain's quote stems from 1897, improving one's health has now become more important than ever. It has been estimated that in 2015, worldwide, as many as 107.7 million children and 603.7 million adults sufficed the obesity criterion of a Body Mass Index (BMI) of 30 or higher (The GBD 2015 Obesity Collaborators, 2017). Obesity has severe medical consequences, such as cardiovascular disease, type 2 diabetes, asthma and obstructive sleep apnoea, as well as serious consequences for the quality of life, including weight discrimination, depression and mobility problems (Finer, 2015; Uzogara, 2017). The worldwide rise in obesity prevalence rates, across age-groups and levels of education, urgently calls for effective interventions to reduce weight (e.g., Flegal, Kruszon-Moran, Carroll, Fryar, \& Ogden, 2016; Uzogara, 2017). The fact that weight loss interventions that aim purely at a negative energy balance (i.e., less energy intake than energy expenditure) rarely lead to successful long-term weight loss fuels the search for effective interventions (e.g., Curioni \& Lourenco, 2005; Franz et al., 2007). In the present dissertation, it is investigated whether food cue exposure therapy can be used as a weight loss intervention, by investigating the effects and potential working mechanisms of cue exposure therapy.

Weight gain due to excessive caloric intake for hedonic purposes instead of eating to fulfil physiological needs seems to be a core problem of obesity, also referred to as eating in the absence of hunger (EAH; e.g., Butte et al., 2007; Kral et al., 2013; Lowe \& Butryn, 2007). In addition, binge eating, defined as eating an unusually large amount of food within a discrete period of time accompanied by the feeling of loss of control (American Psychiatric Association, 2013), is a common problem among obese people (Marcus, Wing, \& Lamparski, 1985; Mustelin, Bulik, Kaprio, \& Keski-Rahkonen, 2017; Telch, Agras, \& Rossiter, 1988) and also associated with future weight gain (Sonneville, 2012; Tanofsky-Kraff et al., 2009).

Food-related cues are theorized to play an important role in EAH and binge eating, by initiating so-called food cue reactivity (Jansen, 1998). Exposure to environmental food cues initiates physical reactions that serve as a preparation for food digestion (e.g., salivary production, insulin rise), while food cues also initiate psychological reactions (i.e., food cravings), altogether labelled as food cue reactivity (Jansen, 1998). Although food cue reactivity is generally an adaptive response to prepare for food intake (e.g., Jansen et al., 2011; Nederkoorn, Smulders, \& Jansen, 2000), increased food cue reactivity might be a major obstacle for resisting palatable foods and successful weight loss (Jansen, Schyns, Bongers, \& van den Akker, 2016). For example, food cue reactivity is higher in overweight versus normal weight individuals (Ferriday \& Brunstrom, 2011), and it is significantly correlated with food intake in overweight children (Jansen et al., 2003). Moreover, a recent 
meta-analysis showed that the magnitude of induced cue reactivity (i.e., cue-induced eating desires) is prospectively related to the amount eaten in a laboratory setting and weight, measured both immediately after the cue reactivity manipulation and at longer-term (i.e., one week - two years), with similar (medium) effect sizes for children and adults (Boswell \& Kober, 2016). Further, a pilot study compared food cue reactivity between obese dieters and successful dieters who were previously obese, successfully lost weight and maintained the weight loss for half a year. Results showed that salivary responses to palatable food pictures were significantly reduced in successful dieters compared to obese dieters (Jansen, Stegerman, Roefs, Nederkoorn, \& Havermans, 2010). Decreasing food cue reactivity might thus make it easier to refrain from eating high-caloric foods, and is therefore a potentially important target to achieve successful weight loss.

\section{THE ROLE OF CLASSICAL CONDITIONING IN FOOD CUE REACTIVITY}

Food cue reactivity might (at least partly) be learned through classical conditioning (Jansen, 1998; Jansen et al., 2016): When food intake (unconditioned stimulus; US) is regularly coupled with specific cues such as the smell or sight of food (conditioned stimuli; CS), these cues can become food-associated and, after repeated CS-US pairings, they will be capable of initiating food cue reactivity (conditioned responses; CR). Several human conditioning studies indeed found that neutral cues can easily become associated with food intake and acquire the capability of initiating conditioned eating desires (Bongers, Jansen, Bongers, \& Jansen, 2017; Bongers, van den Akker, Havermans, \& Jansen, 2015; van den Akker, Havermans, Bouton, \& Jansen, 2014; van den Akker, Havermans, \& Jansen, 2015; van den Akker, Jansen, Frentz, \& Havermans, 2013; van den Akker, van den Broek, Havermans, \& Jansen, 2016; Van Gucht, Vansteenwegen, Van den Bergh, \& Beckers, 2008). While neutral objects are used in conditioning studies, any cue can in principle become associated with food intake, such as the time of day, rituals, cognitions, mood states and even a state of satiation. Take for example the case of Vivian:

"Vivian just had her first baby, who is now 11 months old and has troubles falling asleep. Every other evening, when she is exhausted and her baby is finally asleep, she treats herself to one episode of her new favorite Netflix series. During the first few episodes, she always eats a few pieces Tony Chocolonely chocolate (US). By the time she reaches the sixth episode, turning on Netflix (CS) already increases her chocolate desires (CR), and this strong urge inevitably leads to eating the chocolate. Despite her intentions to take only two small pieces, she oftentimes ends up with eating half the bar, or more... She feels really unhappy about this; how will she ever lose the extra weight from her pregnancy?!"

Given the abundance of food-related cues in our so-called obesogenic environment, we daily encounter many opportunities in which such cue-food intake (CS-US) associations can easily be formed. If CS-US associations are indeed acquired in daily life through 
classical conditioning, extinction learning may be able to reduce these conditioned eating desires. In extinction learning, the CSs are no longer coupled with the US (i.e., food intake), which - after many trials - results in decreased associative strength between the CSs and the US, and decreased conditioned eating desires. In human conditioning studies (e.g., van den Akker et al., 2014, 2015), in which the participant first receives an acquisition phase during which a stimulus is coupled with food intake, extinction of conditioned food desires is achieved by presenting the food-associated stimulus for several trials (e.g., 12 trials used by van den Akker et al., 2016), without receiving the food.

\section{FOOD CUE EXPOSURE THERAPY}

Food cue exposure therapy is the clinical translation of extinction: to expose participants to CSs during a number of sessions, while food intake (US) is prevented. Before commencing therapy, the participant is carefully asked which foods are regarded as highly palatable and are frequently overeaten. The participant receives large portions of these foods, and is instructed to look at the food, smell, hold and taste it (CSs), while not being allowed to eat the food (no US). In addition, other relevant CSs can be included in these exercises as well, such as doing exposure at the food-associated time of day or mood state. In Vivian's case, cue exposure exercises would entail exposing her to a Tony Chocolonely bar, including opening the package, smelling, holding and tasting the chocolate while having her Netflix series on (CSs), and without actually eating the chocolate (no US). At the end of the cue exposure session, food is either thrown away in a garbage bin or preserved for a later exposure exercise.

Small studies in bulimia nervosa patients show that cue exposure therapy leads to substantial reductions of food cravings and binge eating (Bulik, Sullivan, Carter, McIntosh, \& Joyce, 1998; Jansen, Broekmate, \& Heymans, 1992; Jansen, Van den Hout, De Loof, Zandbergen, \& Griez, 1989; Martinez-mallen et al., 2007; Rosen \& Leitenberg, 1982; Schmidt \& Marks, 1988; Toro et al., 2003). With regard to overweight samples, Boutelle and colleagues (2011) investigated an eight-session cue exposure therapy in obese children, and found that cue exposure treatment was more effective in decreasing EAH compared to an appetite awareness intervention at post-treatment, though differences between conditions were no longer significant at six months post-treatment. In another study, cue exposure and appetite awareness were combined in a 16-session intervention for obese children, which had no significant effect on EAH directly after therapy compared to a no treatment control group, but significantly less EAH was found at follow-up (Boutelle et al., 2014). Though cue exposure has been found to decrease EAH in obese children, it is of interest to investigate the specificity of cue exposure effects on EAH: Do participants eat less of foods that were specifically included in exposure therapy (i.e., exposed foods), and do exposure effects also generalise to other non-exposed foods? While studying the specificity effect is 
highly important for the clinical utility of food cue exposure, to date, the effects of food cue exposure on overeating of exposed versus non-exposed foods has not been investigated and will therefore be a central question in this dissertation.

In addition to investigating the effects of cue exposure therapy on overeating (EAH), it is of interest to investigate whether cue exposure therapy also leads to less binge eating in obese people, and ultimately, to weight loss. Early research suggests that cue exposure can successfully prevent weight gain (Mount, Neziroglu, \& Taylor, 1990). Participants who received cue exposure therapy after an intensive weight loss program successfully maintained their weight loss, while participants receiving a stimulus control intervention (i.e., avoidance of food cues) regained the lost weight. A combined cue exposure and appetite awareness therapy (see Boutelle et al., 2014) was also investigated in in overweight and obese binge eating adults. The intervention led to a lower BMI after 14 sessions, also at 3-months follow-up, but note that this study did not include a controlgroup. Binge eating was marginally reduced during therapy, but at follow-up, binge eating frequency no longer differed significantly from pre-measurement (Boutelle, Knatz, Carlson, Bergmann, \& Peterson, 2016).

In sum, studies so far suggest that cue exposure might be a valuable intervention to reduce food cravings, EAH and binge eating, while there is also some indication that cue exposure might promote weight loss. However, especially in overweight samples, the available research on cue exposure therapy is limited, and effects of pure cue exposure therapy on overeating, binge eating and weight loss are largely undiscovered. While there are a few studies on the effects of food cue exposure, so far, food cue exposure's working mechanisms have never been studied and therefore form another central issue in this dissertation.

\section{WORKING MECHANISMS OF FOOD CUE EXPOSURE THERAPY}

\section{HABITUATION}

For a long time, habituation has been promoted as the working mechanism for exposure therapy for anxiety disorders, based on Emotional Processing Theory (Foa \& Kozak, 1986; Foa \& McNally, 1996). The prevailing idea is that exposure sessions should activate the 'fear structure' in memory and aim at habituation of fear within exposure sessions (WSH), and between exposure sessions (BSH), predicting that the treatment success depends on sufficient WSH and BSH (Foa \& Kozak, 1986; Foa \& McNally, 1996). However, Craske and colleagues (2008) pointed out that neither of these habituation parameters are consistently related to better treatment outcome. A study in acrophobic students, for example, investigated the relationship between habituation and fear responding during a behavioural avoidance test and scores on the clinical anxiety scale. Results showed that 
WSH of self-reported fear and heart rate did not predict any outcome measure, neither directly after therapy nor at the two-week post-measurement. While BSH was predictive of some treatment outcomes directly after therapy, it generally did not predict outcome two weeks later (Baker et al., 2010). The Emotional Processing Theory would also predict that stopping exposure at a high point of anxiety cannot be effective. Rachman and colleagues (1986) instructed agoraphobic participants in an 'escape condition' to stop the exposure when their anxiety reached 70 on a 100-point scale, while others continued the exposure until their anxiety dropped by $50 \%$ of the peak fear. Participants in the escape condition improved as much as the habituation participants, a finding that is not predicted by the emotional processing theory.

In all food cue exposure studies so far, cue exposure interventions were designed according to the habituation model. That is, cue exposure sessions were aimed at sufficient habituation of arousal or food cravings elicited by the cues (CS), as quantified by reaching an arousal level of 10 or lower on a 100-point arousal scale (Mount et al., 1990), or a score of 2 or lower on a 5-point craving scale (Boutelle et al., 2011, 2014, 2016). Others aimed at the habituation of provoked anxiety to determine the end of food cue exposure sessions in eating disorders (Martinez-mallen et al., 2007), and patients would receive as many sessions as needed to reduce anxiety levels to a level of four or lower on a 10-point scale (Toro et al., 2003). Given that habituation is not consistently related to better treatment outcome in anxiety disorders, an important question therefore is whether habituation is a useful target for food cue exposure sessions.

\section{INHIBITORY LEARNING}

Fundamental conditioning studies have convincingly shown that even though extinction procedures can successfully reduce conditioned responses, conditioned responses can return (Bouton, 1993; Bouton \& King, 1983). Several phenomena illustrate the specific conditions in which responses return after extinction, such as spontaneous recovery (i.e., return of conditioned responses after the passage of time), renewal (i.e., return of conditioned responses after a context switch), reinstatement (i.e., return of conditioned behaviour after presentation of the US), and rapid reacquisition (i.e., the return of responses after exposure to CS-US pairings; e.g., Bouton, 2011). Given the large body of research on the return of conditioned responses, it is now well-established that extinction learning is not erasing original CS-US learning, but instead it is proposed that the CS acquires a secondary inhibitory meaning, namely that CS means no US. After extinction, the CS is ambiguous. That is, while the CS continues to signal the US, it is now also associated with no US. According to this inhibitory learning model, exposure therapy is most effective when the new CS-noUS association becomes stronger than the old CS-US association, that is, when inhibitory learning is strong (Bouton, 1993; Craske et al., 2008). 
Although CS-US associations involved in anxiety are content-wise distinct from CS-US associations involved in overeating, learning principles in the acquisition and extinction of such associations might essentially be the same. Peters and colleagues (2009) demonstrated that an overlapping neural circuitry is involved in the extinction of fear and reward memories, indicating a common extinction mechanism, and the possibility of a transdiagnostic treatment approach (exposure) for anxiety and overeating. Based on the theory of inhibitory learning, Vivian will thus never forget the Netflix - chocolate (CS-US) association, but food cue exposure therapy is aimed at creating a new and stronger Netflix - no chocolate (CS-noUS) association.

\section{EXPECTANCY VIOLATION}

Craske and colleagues (2014) have described several strategies to enhance inhibitory learning during exposure therapy for anxiety disorders. One strategy is to target exposure sessions at the violation of US expectancies. For example, targeting the expectancy that a heart attack will occur in case of panic disorder. To achieve maximal violation of expectancies, therapists should carefully introduce CSs (e.g., high heart-rate, sweating) that maximize the mismatch between the expectancy of the US to take place (e.g., heart attack) and the actual outcome (e.g., the absence of the heart attack; noUS), thereby strengthening the CS-noUS association.

Results of a pilot study in 16 panic disorder patients with agoraphobia showed that patients who received two exposure sessions targeted at expectancy violation reported fewer panic attacks, and less anxiety and avoidance of fearful situations, compared to patients who received two exposure sessions targeted at habituation of fears (Salkovskis, Hackmann, Wells, Gelder, \& Clark, 2006). While the amount of exposure (3.25 hours) was kept constant for both conditions, patients in the expectancy violation condition also dared to face more fearful situations in a behavioural walk task compared to patients in the habituation condition (Salkovskis et al., 2006). Others found that an intensive exposure condition that continued exposure therapy until the expectancy of the feared outcome was $5 \%$ or less was superior to two lower-dose exposure therapy conditions that ended at higher expectancies (Deacon et al., 2013). In this study, the amount of exposure was much longer in the intensive exposure condition compared to the lower-dose conditions, though, interestingly, the superior treatment outcome in the intensive exposure condition compared to the other conditions was fully mediated by the violation of danger expectancies during exposure (Deacon et al., 2013).

CS-US expectancies may also play a significant role in overeating, such as 'If I feel exhausted and chocolate is available (CS), then I will lose control and eat the entire chocolate bar (US)', or 'If my family is eating a snack, then I simply must join them (US)'. The expectancy violation approach could therefore be very suitable to use in cue exposure sessions in people with obesity and/or binge eating. Following this approach, cue exposure 
sessions should be designed by introducing specific overeating cues that are linked to strong eating expectancies, while testing whether the US indeed takes place as expected. For example, when designing a cue exposure session to challenge the expectancy 'If I feel exhausted and chocolate is available (CS), then I will lose control and eat the entire chocolate bar (US)', it should be pre-specified under which exact conditions (i.e., which CSs) it is most likely that the participant will lose control (US). Cue exposure, including those specific CSs, should then continue until the point that losing control is no longer expected. While expectancy violation does seem well-translatable to food cue exposure, no cue exposure studies have been conducted using expectancy violation as a target for exposure sessions, or have compared this new approach to the traditional habituation approach. Therefore, the comparison of both approaches is a central issue in the present dissertation.

\section{OTHER STRATEGIES TO ENHANCE INHIBITORY LEARNING}

While expectancy violation has been recommended as a strategy to increase inhibitory learning during exposure therapy, other techniques have been proposed as well to avoid specific types of relapse (Craske et al., 2014). Some of these techniques seem to be especially relevant for the food domain, and will be discussed in the following section.

As shortly mentioned above, one important risk factor for the return of appetitive responses is a change of context. Specifically, a vast amount of fundamental research has shown that extinction learning is context-dependent, in contrast to the original excitatory association that easily generalises to other environments and circumstances (for reviews, see Bouton, 1988, 2011). Specifically, during extinction, one does not learn that the CS no longer predicts the US in general, but rather learns that the CS no longer predicts the US in the specific extinction context. The relapse of behaviour due to context change is called renewal, and can for example occur when exposure therapy is successfully completed in a therapy setting and food cravings return when returning home. One solution to limit renewal is to extend extinction learning to other relevant contexts. This was for example found in a spider exposure therapy experiment, in which spider phobic patients were exposed to spiders in either one virtual reality context, or four virtual reality contexts. At test, when participants viewed a spider in a novel context, renewal was significantly lower in the multiple contexts condition compared to the single contexts condition (Shiban, Pauli, \& Mühlberger, 2013). For food cue exposure therapy, it therefore seems worthwhile to do cue exposure exercises at the participant's home and other contexts where overeating takes place, for the CS-noUS association to generalise to other relevant overeating contexts.

Doing exposure in multiple contexts, however, is not always possible due to practical considerations. A different solution to diminish renewal could be to provide participant's with 'reminders' of extinction: retrieval cues. Retrieval cues can be physical cues that have been introduced during extinction, such as pens or bracelets that are specifically linked to exposure therapy, but can also be mental cues by actively remembering what the participant 
has learned during therapy (Craske et al., 2014). Adding a physical retrieval cue attenuates renewed responding after a context switch in conditioning studies (Dibbets, Havermans, \& Arntz, 2008; Vansteenwegen et al., 2006), though results in more clinically applied exposure studies generally failed to show significant effects (Culver, Stoyanova, \& Craske, 2011; Dibbets, Moor, \& Voncken, 2013). Mental retrieval cues on the other hand seem to be somewhat more promising. Mystowksi and colleagues (2006) found that after exposure therapy for fear of spiders, participants who were instructed to remember the exposure session reported less return of fear after a context switch compared to participants who were instructed to think of a neutral memory, though these findings were limited to selfreport measures. Similar results were found in an exposure study on dental phobia, in which patients received an exposure session at a psychology unit and were tested one week later at a dental clinic. Patients who mentally retrieved the treatment session showed less avoidance in picking up a forceps during a behavioural test as compared to participants in a control condition who were instructed to retrieve of a neutral memory, but no differences were found on avoidance of two other dental instruments, heart-rate and self-reported anxiety during the behavioural test, or on general anxiety questionnaires (Elsesser, Wannemüller, Lohrmann, Jöhren, \& Sartory, 2013).

Relapse can be caused by a context switch, but also by reinforcement of the US (i.e., reinstatement) and original CS-US association (i.e., rapid reacquisition) in the future. These types of relapse seem especially important to take into account in cue exposure therapy for overeating, as appetitive associations, in contrast to associations in anxiety disorders, are likely to get reinforced again in the future (Bouton, 2011). In the case of Vivian: It is likely unrealistic to assume that she will never eat Tony chocolonely chocolate again (US reinforcement), or never eat chocolate again when watching Netflix (CS-US reinforcement) in the future. Instead, she needs to learn that eating a bit of chocolate on one night does not lead to eating chocolate every following night, and that eating one piece of chocolate does not lead to eating the entire bar. Prevention of this type of relapse can be achieved by introducing occasional US reinforcements during the extinction procedure. In practice this means that during cue exposure exercises, participants are occasionally required to take a small bite of the exposed food. In this exercise, participants learn that eating a small piece of chocolate predicts no further intake of chocolate, and therefore does not lead to a full-blown relapse of eating desires (Bouton, 2011; Craske et al., 2014). Evidence for this extinction technique was obtained in an experiment with rats, showing that occasionally reinforcing the CS-US association during extinction led to a reduced return of conditioned responses during the reacquisition phase (i.e., less rapid reacquisition), in which the CSs are again paired with USs, compared to a regular extinction procedure (Bouton, Woods, \& Pineño, 2004). Similar results were found in a human conditioning experiment, in which an occasional reinforced extinction condition experienced slower reacquisition of eating expectancies compared to a regular extinction condition, though no differences were found 
for reacquisition of eating desires (van den Akker et al., 2015). To date, no cue exposure studies have incorporated occasional reinforcement during exposure therapy.

\section{THIS DISSERTATION}

Taken together, the limited number of studies so far suggest that food cue exposure might be a valuable intervention to reduce overeating and binge eating, though it is unknown whether cue exposure reduces overeating of exposed foods specifically, and whether exposure effects also generalise to non-exposed foods. Secondly, working mechanisms of food cue exposure have never been studied. Research in the anxiety domain indicates that exposure sessions are most effective when aimed at maximizing inhibitory learning. It has been suggested that aiming at violation of US expectancies leads to better treatment outcomes than aiming at habituation of fears - raising the question whether this also holds for food cue exposure. Finally, and importantly, very little is known about whether cue exposure therapy can promote weight loss. The aim of this dissertation is therefore to investigate the effects and working mechanisms of food cue exposure for overweight and obese participants, by answering three central questions:

1) Does food cue exposure reduce the overeating of foods used in cue exposure exercises (i.e., exposed foods; 1a), and does it generalise to non-exposed foods (1b)? The studies described in chapters 2, 3, 5 and 6 are designed specifically to answer this question, hypothesising that cue exposure significantly reduces overeating of exposed and non-exposed foods.

2) Can the effects of food cue exposure on treatment outcomes be explained by habituation of cue reactivity, or by violation of US expectancies? It is hypothesised that treatment outcomes are positively associated with WSH and $\mathrm{BSH}$ of cue reactivity if habituation is critical for extinction, whereas outcomes are expected to be related to the strength ofCS-US expectancies if expectancy violation is critical. Both habituation and expectancy violation are investigated in chapters 2, 3, and 5, and experimentally manipulated in chapter 6 .

3) Can food cue exposure therapy be helpful to decrease overeating, binge eating, and can it be used to achieve weight loss? It is expected that food cue exposure therapy will induce stronger reductions in overeating, binge eating, and induce more weight loss, compared to an active control intervention aimed at improving lifestyle. Techniques aimed at inhibitory learning in food cue exposure (e.g., multiple contexts, mental retrieval cues, occasional reinforcements) are described in chapter 4 , and the effects of an eight-session food cue exposure intervention containing several inhibitory learning techniques is examined in chapter 5 .

In chapter 7, the data from the studies described in chapters 2, 3, 5 and 6 are aggregated into one dataset to answer the first and second central questions above. Finally, in chapter 8 , answers to the central questions will be summarized and discussed. Recommendations for cue exposure therapy in clinical practice and directions for future research will be provided. 



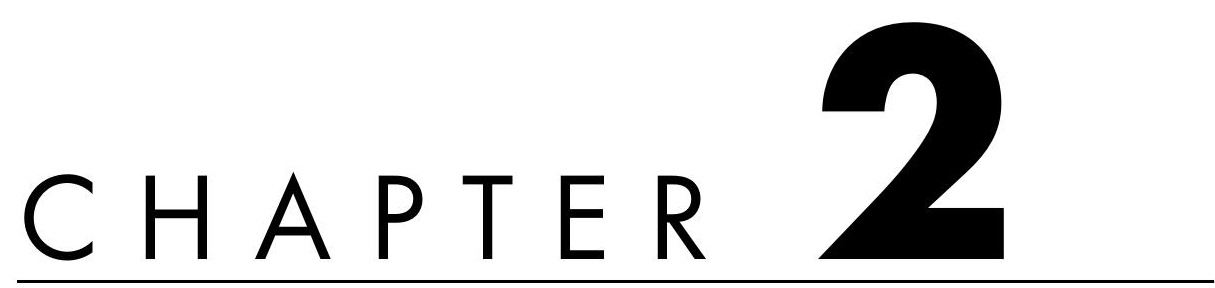

\section{EXPECTANCY VIOLATION, REDUCTION OF FOOD CUE REACTIVITY AND LESS EATING} IN THE ABSENCE OF HUNGER AFTER ONE FOOD CUE EXPOSURE SESSION FOR OVERWEIGHT AND OBESE WOMEN

Published as:

Schyns, G., Roefs, A., Mulkens, S., \& Jansen, A. (2016). Expectancy violation, reduction of food cue reactivity and less eating in the absence of hunger after one food cue exposure session for overweight and obese women. Behaviour Research and Therapy, 76, 57-64. https://doi.org/10.1016/j.brat.2015.11.007. 


\section{ABSTRACT}

The present study investigated whether a single-session of food cue exposure for overweight women would decrease 'if CS then US' expectancies, cue reactivity and eating in the absence of hunger (EAH). EAH was measured in a behavioural paradigm that enabled to also investigate whether the cue exposure effects were specific for exposed foods or would generalise to food items that were not present during exposure. Overweight women were randomly assigned to either the cue exposure intervention or a control intervention that focused on body image. In line with the hypotheses, results showed that cue exposure induced a significant decrease in 'if CS then US' expectancies, in contrast to the control intervention. It was also found that, compared to the control intervention, desires to eat initially increased during cue exposure while gradual extinction was observed towards the end of the intervention. No extinction of increased salivation responses was found. Regarding EAH, the intake of the exposed food item was significantly less in the exposure condition than in the control condition, whereas total caloric food intake was not different between conditions, indicating that cue exposure was effective in reducing intake but did not generalise to the intake of other food items.

KEYWORDS: food cue exposure, extinction, obesity, cue reactivity, eating in the absence of hunger, body image 


\section{INTRODUCTION}

Overweight and obesity prevalence's pose a serious problem worldwide. In the Unites States, more than two-third of the adult population is overweight of which approximately half is obese (Ogden, Carroll, Kit, \& Flegal, 2014). In European countries, as much as $50 \%$ of the adults population is overweight, including obesity prevalence's of $20 \%$ in men and $23 \%$ in women (WHO, 2014). Eating more than needed is primarily caused by hedonic eating or, put differently, eating for pleasure in the absence of (physiological) hunger (Lowe \& Butryn, 2007). A cause of eating in the absence of hunger (EAH) is increased reactivity to food cues (Jansen, Havermans, \& Nederkoorn, 2011a; Jansen et al., 2003). Food cue reactivity refers to anticipatory bodily reactions that prepare for food intake, such as saliva production, gastric activity and insulin rise, as well as psychological reactions such as a strong desire to eat the food (e.g. Jansen, 1998; Jansen et al., 2011a). Jansen (1998) theorized that most cue reactivity is classically conditioned. In classical conditioning, neutral stimuli (conditioned stimuli; CS) can become associated with food intake (unconditioned stimulus; US) and, after a process of classical conditioning, just the confrontation with CSs can elicit conditioned responses (CR) as preparation for food digestion (Pavlov, 1927). With regard to human cue reactivity, stimuli such as the smell and sight of food (CS) that acquire predictive value of food intake (US) will easily elicit cue reactivity (CR), including increased eating desires and salivation (Jansen, 1998). Indeed, conditioning studies in animals and humans have found that after repeated pairings of neutral stimuli (CS) with food intake (US), CSs are able to elicit increased eating desires and bodily responses to prepare for food intake (e.g. salivation), and may stimulate overeating (e.g. Boggiano, Dorsey, Thomas, \& Murdaugh, 2009; Bouton, 2011; van den Akker, Havermans, Bouton, \& Jansen, 2014; van den Akker, Havermans, \& Jansen, 2015; van den Akker, Jansen, Frentz, \& Havermans, 2013). In line with these findings, it was found that healthy-weight non-restrained eaters showed increased gastric activity, heart rate and saliva production during exposure to food cues (CSs) (Nederkoorn \& Jansen, 2002).

According to Jansen's (1998) model, increased cue reactivity makes it harder to resist palatable food. This was indeed confirmed by a study in healthy-weight students, who received 10-minute exposure to food cues, and on a separate day, a 10-minute control task. Food intake was measured after both tasks. Results showed that students consumed more food after food exposure the control task (Jansen et al., 2011b). Ferriday and Brunstrom (2011) investigated cue reactivity and food intake in healthy-weight and overweight individuals, and found that one minute of food exposure led to increased desire to eat and food intake in both groups. However, food exposure led to significantly larger salivation and desire to eat in the overweight individuals as compared to the healthy-weight individuals. Along the same lines, a study that compared 10-minute food exposure in overweight 
children versus healthy-weight children found that overweight children overate after food cue exposure, compared to healthy-weight children who successfully controlled their food intake. Moreover, food cue-induced salivary flow in the overweight children, and not in the healthy-weight children, was significantly positively related to food intake (Jansen et al., 2003). These findings show that exposure to food cues increases cue reactivity and food intake in healthy-weight participants - it is a normal response - while the effects are significantly stronger in overweight participants, making it more difficult for overweight people to resist tasty foods.

Circumstantial evidence for the hypothesis of cue reactivity playing a role in overeating comes from a pilot cue reactivity study in successful dieters (formerly obese) and unsuccessful dieters (still obese). The successful dieters had a current $\mathrm{BMI}<25$, had achieved a mean weight loss of $27 \%$ of their original body weight and had maintained this weight loss for at least half a year, whereas unsuccessful dieters had a current BMI of $>30$ despite serious weight loss attempts. It was found that the unsuccessful dieters displayed a significant increase in salivation response to tasty food pictures relative to baseline salivation, whereas the successful dieters showed a significant decrease in salivation (Jansen, Stegerman, Roefs, Nederkoorn, \& Havermans, 2010). These findings suggest that successful refraining oneself from high-caloric foods eventually may result in decreased cue reactivity. As the authors suggested, decreased cue reactivity might, in turn, make it easier to resist foods and to maintain the weight loss. Reducing cue reactivity could therefore be helpful to lose weight and to successfully prevent relapse. In cue exposure, the clinical proxy of extinction, participants are exposed to non-reinforced CSs predicting US, in order to decrease cue reactivity (CR). For example, participants smell, touch and lick the tasty foods (CSs) while actual food intake (US) is not permitted. Some small clinical studies with bulimia nervosa patients suggest that cue exposure can be very effective in reducing food cravings and binge eating (Jansen, Broekmate, \& Heymans, 1992; Jansen, Van den Hout, De Loof, Zandbergen, \& Griez, 1989; Martinez-malle et al., 2007; Toro et al., 2003). A clinical study by Boutelle and colleagues (2011) showed that an 8-session cue exposure treatment in obese children and their parents was more effective to decrease EAH compared to an appetite awareness intervention. In another, 16-session intervention study in which cue exposure and appetite awareness components were combined, no direct effects after therapy were found on EAH compared to a no treatment control group, but significant lower EAH was found at followup (Boutelle et al., 2014). Though food cue exposure is a promising intervention that seems to be effective in reducing eating desires and EAH, knowledge about its working mechanisms is still lacking.

It is now generally acknowledged that exposure therapy works through inhibitory learning (Craske et al., 2008; Craske, Liao, Brown, \& Vervliet, 2012). Though it was originally assumed that by exposure to the CS without the occurrence of the US, the original CS - US 
association could be destroyed, this appears not to be the case: extinction does not destroy the original CS - US bound, but creates a new learning pathway; the CS means that the US will not follow (CS - no US). In other words, the CS acquires two meanings: CS means US and CS means no US (Bouton, 1993; Bouton \& King, 1983). Exposure should aim to make the new association (CS - no US) stronger than the old association (CS - US). Exposure should therefore be designed to optimally learn the new CS - no US association, that is, the client learns that the US will probably not occur in the presence of the CS. This is also called CS - US expectancy violation (Craske et al., 2012; Craske, Treanor, Conway, Zbozinek, \& Vervliet, 2014). Craske and colleagues $(2012 ; 2014)$ argue that CS - US expectancy violation during exposure is the key element for exposure to be effective: the more violation, the more treatment effect. This approach contrasts the habituation model, in which the central idea is to stay in the situation until fear (or in this case: the desire to eat) declines. Indeed, habituation either within and between exposure trials and ending fear levels have shown not to be a good predictor for treatment outcome (for review see Craske et al., 2008).

The primary aim of the present clinical experiment is to investigate whether food cue exposure is effective in reducing 'if CS then US' expectancies (i.e., expectancy violation), cue reactivity (saliva production and self-reported desires to eat), and eating in the absence of hunger (EAH) in overweight and obese females. 'If CS then US' expectancies are implicitly but not explicitly challenged in the present experiment. It is hypothesised that cue exposure significantly reduces 'if CS then US' expectancies, compared to the control condition (Rijkeboer \& Van den Hout, 2014). Regarding cue reactivity, it is expected that cue exposure significantly increases salivation and the desire to eat in the beginning of the exposure, followed by extinction during prolonged exposure. The cue exposure condition is further hypothesized to show less eating in the absence of hunger (EAH) compared to the control condition, both for the exposed food item chocolate mousse and total consumption (generalisation). Finally the role of habituation vs. inhibition learning regarding treatment outcome (EAH) is investigated: EAH is expected to be positively associated with postintervention 'if CS then US' expectations if inhibition learning is critical, whereas EAH is expected to be related to within-session habituation (WSH) of cue reactivity if habituation is critical for extinction.

\section{METHOD}

\section{PARTICIPANTS}

Participants who were motivated to lose weight were recruited via posters that were displayed in supermarkets and gyms. Fifty-four overweight women (Body Mass Index > 
25) aged between 18 and 65, who were not pregnant, and were able to smell, participated in this study. Participants were told that the study's aim was to investigate the effects of a single-session psychological training to cope with food temptations. Participants were randomly assigned to the cue exposure condition $(n=26)$ or the control condition $(n=$ 28 ), by assigning the first six participants who could make it on the same intervention date to the cue exposure condition, the six next participants to the control condition, and so on. The study was approved by the Ethical Committee of the Faculty of Psychology and Neuroscience of Maastricht University.

\section{ASSESSMENT}

'If CS then US' expectancy: The following 'if CS then US' statement for overeating was used: 'If I have tasty food in front of me, then I can not resist to eat it'. The believability of this statement at the present moment was rated on a 0 (not at all) to $100 \mathrm{~mm}$ (very strong) Visual Analogue Scale (VAS).

Desire to eat: Desire to eat was measured by the statement: 'At the present moment, I have.... The statement was answered by rating a 0 (no desire at all to eat tasty food) to 100 $\mathrm{mm}$ (an extreme desire to eat tasty food) VAS. Desire to eat VASs are supposed to be valid measures of eating desires (e.g. Bongers, van den Akker, Havermans, \& Jansen, 2015; van den Akker et al., 2014; van den Akker et al., 2015; van den Akker et al., 2013).

Hunger: Current hunger was measured by the statement: 'At the present moment, I am..., and answered by rating a 0 (not hungry at all) to $100 \mathrm{~mm}$ (very hungry) VAS.

Salivation: Salivation was measured by placing two dental cotton rolls (Hartmann, nr2, 10x35 mm) in the mouth between the cheek and left and right lower gums. The cotton rolls stayed in the mouth for exactly one minute. They were placed and removed by the participant and kept in a sealed plastic bag that was weighed before and after the measurement on a $0.01 \mathrm{~g}$ accurate weighing scale (Mettler Toledo, PB3002).

EAH behavioural paradigm: The EAH paradigm was adapted from the paradigm described by Birch and Fisher (2000). In the present study, each participant received two standard pre-packed sandwiches that she was required to eat, to induce a state of satiation (absence of hunger). A questionnaire on taste and quality was provided together with the sandwiches, as a first part of the bogus taste test. After finishing the sandwiches, participants waited for 15 minutes to achieve a satiated state. Before and 15 minutes after the required standard meal, current hunger was measured. Directly after the post meal hunger rating, a 10-minute bogus taste test of 6 desserts started. Participants were told that this test was to assess taste perception of six different food items. Participants were instructed to rate the taste of each of the 6 desserts (using a questionnaire) while being allowed to eat as much as they wanted from the generous portions of each food item: chocolate mousse $(\sim 80$ g/ $142 \mathrm{kcal}$; Almhof), whipped cream ( 90g/ 165 kcal; Campina), muffin ( $~ 80 \mathrm{~g} / \sim$ 
$262 \mathrm{kcal}$; Jumbo supermarket), custard ( $190 \mathrm{~g} / \sim 173 \mathrm{kcal}$; Campina), strawberry mousse $(\sim 120 \mathrm{~g} / \sim 156 \mathrm{kcal}$; Dr. Oetker), chocolate cake ( $\sim 80 \mathrm{~g} / \sim 330 \mathrm{kcal}$; Coolmore). The total plate of desserts consisted of roughly $1230 \mathrm{kcal}$. The taste test was done individually; tables were separated by large screens. After the bogus taste test, foods were removed. Each food item was weighed before and after the taste test, and the number of consumed kcal was calculated.

Expectation and evaluation of the intervention. Each participant rated pre- and post intervention how much she expected to learn / had learned from the intervention on a 5-point scale from 1 (not at all) to 5 (very much) and whether she expected the intervention to help her gaining control over eating, rated from 1 (much less control) to 5 (much more control).

Body satisfaction: Body satisfaction was measured with the statement: 'At the present moment, I am.... The statement was answered by rating a 0 (very dissatisfied with my body) to $100 \mathrm{~mm}$ (very satisfied with my body) VAS. Body satisfaction VASs have been used in previous studies (e.g. Jansen et al., 2016).

Pre-intervention intake: At the intervention day, participants were asked to report at what time they had eaten their last meal (breakfast or snack) and what this meal consisted of.

BMI: To calculate BMI $\left(\mathrm{kg} / \mathrm{m}^{2}\right)$, height and weight ( $0.1 \mathrm{~kg}$ accurate; My Weigh, XL-550), were measured in the laboratory by the experimenter.

Awareness check. It was checked whether the participant was aware of the aim of the bogus taste test and study by means of an open question.

\section{PROCEDURE}

Participants gave informed consent, filled out some demographic questions and the favourite food list to determine individualized cue exposure foods online. In order to keep the food appealing during the entire session, warmly prepared foods were not selected as cue exposure foods. The participants were randomly assigned to the cue exposure or the control condition.

Interventions were provided in a group of minimally 3 and maximally 7 participants ( $M$ $=4.5$ participants per group; 12 groups in total). Interventions took place on weekdays from $9.30 \mathrm{am}$ to $1.30 \mathrm{pm}$. Participants were instructed to eat a regular breakfast, but no snacks between breakfast and the intervention. Because of practical considerations, two groups ( 1 cue exposure and 1 control) took place in the afternoon from 13.30 to $17.30 \mathrm{pm}$. For these participants the instruction was to eat a regular lunch, but no snacks between lunch and the intervention.

After arrival in the laboratory, baseline measurements were done (saliva, preintervention intake assessment, and VASs: desire to eat, hunger, body satisfaction, the 
believability of the 'if CS then US' expectancy statement). Then participants were given a short rational behind the intervention they would receive. They rated their expectancy of the intervention, after which the intervention started (see detailed description of interventions below). Both interventions lasted exactly 80 minutes, with breaks in between. In the cue exposure condition, there were three 10-minute breaks after every 20 minutes of exposure, while there was one 30-minute break after 40 minutes in the control condition. Saliva was measured at 13 time-points (at baseline and 12 times during the intervention). Desire to eat was also measured at the same 13 time-points as well as twice after the intervention: before the sandwich meal (BM) and after the meal (AM). In the cue exposure condition, 16 additional desire to eat measurements were taken throughout the intervention, including 4 measurements (minutes 0, 20, 40,60) when exposure foods were not visible. The timer was stopped for interruptions due to the measurements (e.g. placing and removing cotton roles, filling out VASs), resulting in 80 minutes of pure intervention. Eating was not allowed during the interventions and breaks. After the intervention, participants again rated desire to eat, hunger and body dissatisfaction, as well as the believability of the 'if CS then US' expectancy statement. Then the two-sandwich meal was eaten, with coffee or tea, followed by a 15 minutes break and the bogus taste test. Thereafter participants completed the awareness check and participants were weighed and their height was measured. They were thanked for participation and debriefed about the nature of the study, after which they received $€ 25$ for participation. All sessions were audiotaped. An overview of the timing of assessments is displayed in Figure 1.

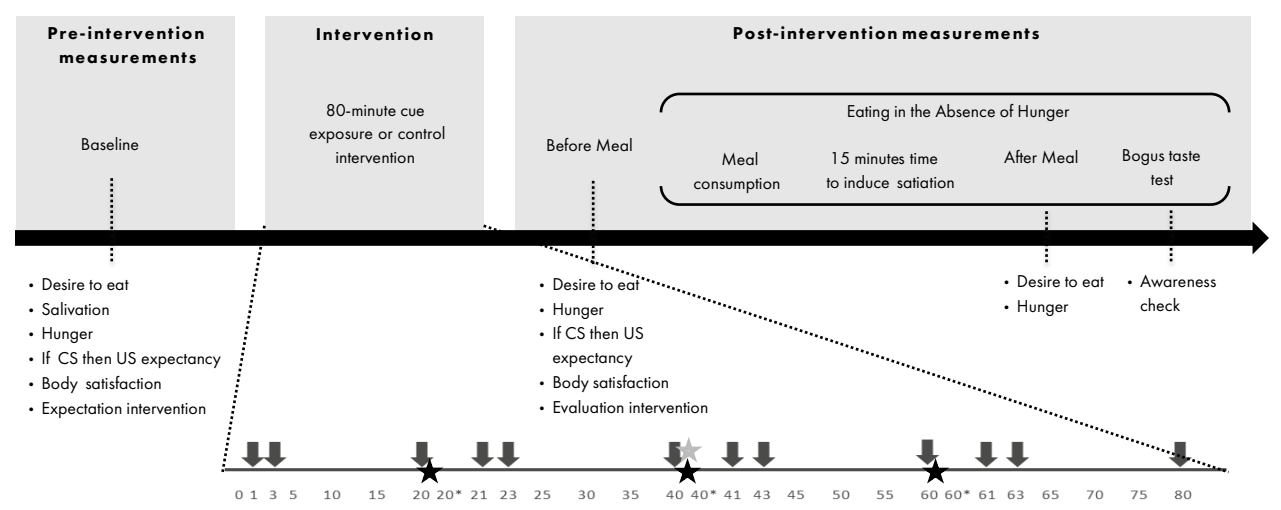

Figure 1. Schematic overview of timing of assessments and intervention. Numbers on timeline reflect minutes of 80-minute intervention on which measurements took place. Minutes marked with $\boldsymbol{\downarrow}$ reflect desire to eat and salivation measurements that were measured in both conditions. Displayed minutes without $\downarrow$ represent additional desire to eat measurements in the cue exposure condition, measurements that were taken when exposure foods were not visible are displayed with *. Black stars represent the 10-minute breaks of the cue exposure condition, the grey star represents the 30-minute break of the control condition. 
Cue exposure intervention. Each participant received their individualized top four favourite food items that were continuously available right in front of them on a tray. Every participant was also exposed to chocolate mousse. Participants were coached in getting their desire to eat the food as high as possible, by smelling (directly under the nose), feeling, licking and imagining eating the food, but consumption was not allowed. Large portions of food were provided, not only to maximise visual and olfactory stimulation, but also in order to have appealing food available during the entire session. For example, several chocolate bars were provided in case the chocolate melted during exposure. During the breaks, tea towels were placed over the food trays. After 80 minutes of food cue exposure, each participant threw away all her foods in a garbage bin.

Control intervention. The aim of the control intervention was to increase body satisfaction, by discussing topics such as the beauty ideal, media literacy and fat talk. The experimenter presented scientific studies and experiments, followed by assignments for the participants that were discussed in the group afterwards. An example of an assignment was to write down the feelings that came up while looking at pictures of healthy-weight and underweight models.

\section{RESULTS}

Descriptives. The mean age of the sample was 44.65 years $(S D=10.91$, range $=21-61)$ with no differences between conditions, $t(52)=1.13, p=.264$. The cue exposure condition $(M=33.89, S D=5.37$, range $: 25.7-52.5)$ had a significantly higher BMI (measured in the laboratory) than the control condition $(M=30.85, S D=3.45$, range : 25.6-37.5), $t(52)=$ $2.49, p=.016$. Including BMI as a covariate was however not a significant contributor for any of the outcome measures, nor was the interaction between group and BMI significant for any of the outcome measures. BMI was therefore not included in the analyses below.

Credibility of the interventions. The mean expectation and evaluation of the intervention on how much will be learned / was learned was equal in the cue exposure condition (preintervention: $M=3.46, S D=0.58$; post-intervention: $M=3.42, S D=0.90$ ) and the control condition (pre-intervention: $M=3.54, S D=0.79$; post-intervention: $M=3.29, S D=0.94$ ), $t(52)=0.39, p=.699$ and $t(52)=0.55, p=.586$ respectively. Regarding control over eating, participants receiving cue exposure (cue exposure: $M=4.23, S D=0.59$ ) had marginally significant higher expectations that the intervention would help them gain control over eating compared to the control condition $(M=3.93, S D=0.54) ; t(52)=1.97, p=.054$. The evaluation scores indicated that, after the intervention, participants rated the cue exposure intervention $(M=4.04, S D=0.53)$ as more helpful in gaining control over eating compared to the control condition $(M=3.71, S D=0.53), t(52)=2.24, p=.029$. 
Lastly, as the control intervention aimed to increase body satisfaction, an ANCOVA was conducted with post-intervention body satisfaction score as dependent variable, condition (cue exposure, control) as independent variable, and pre-intervention body satisfaction score as covariate. Though both conditions scored equal at pre-intervention on the body satisfaction scale (cue exposure: $M=34.00, S D=23.95$; control: $M=33.54, S D=20.71$ ), post-intervention body satisfaction of the control condition $(M=57.54, S D=19.41)$ was significantly higher than post-intervention body satisfaction of the cue exposure condition $(M=39.58, S D=24.63)$, indicating that the control intervention was successful in increasing body satisfaction, $F(1,51)=14.64, p<.001$.

'If CS then US' expectancy. As can be seen in Figure 2, both conditions scored equal on the 'if CS then US' expectancy scale at pre-intervention $(F<1)$ while at post-intervention the cue exposure condition, in contrast to the control condition, believed significantly less in the 'if CS then US' expectancy statement while controlling for pre-intervention 'if CS then US' expectancy in an ANCOVA, $F(1,50)=4.16, p=.047$. These findings indicate that the cue exposure intervention was effective in expectancy violation while the control intervention was not; after cue exposure one's belief in loss of control when confronted with tempting foods was significantly reduced.

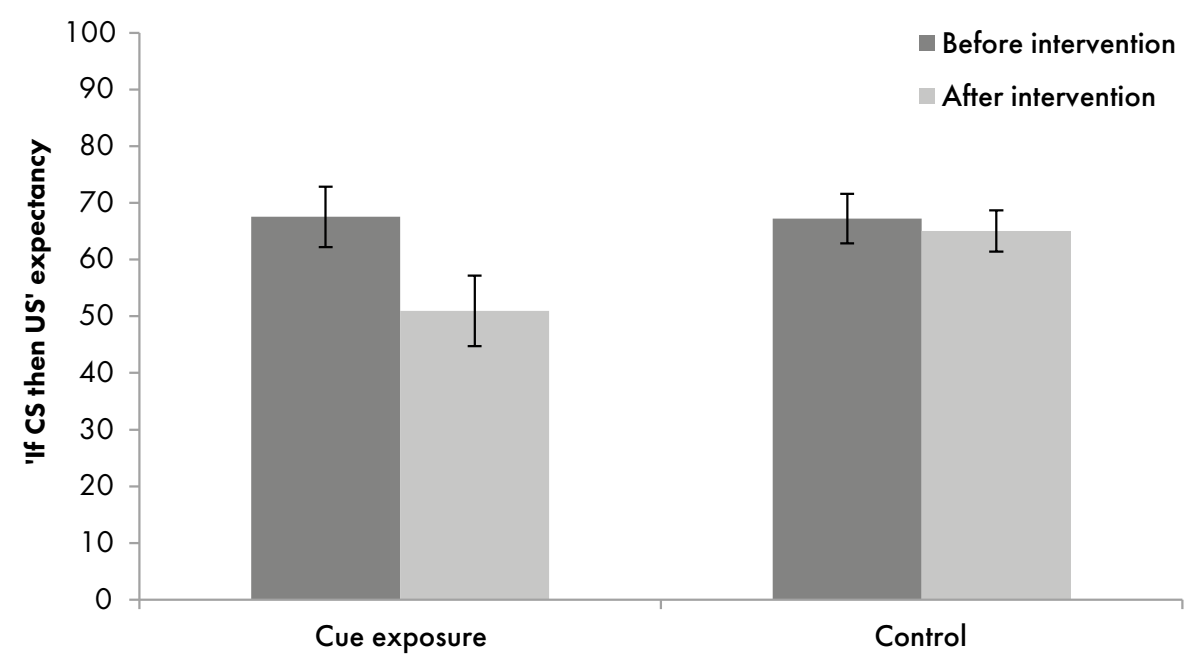

Figure 2. Mean believability of the 'if CS then US' expectancy ('If I have tasty food in front of me, then I can not resist to eat it') score, per condition (cue exposure, control) per time point (before intervention, after intervention). Error bars represent standard errors of means.

Cue reactivity. As can be seen in Figures 3 and 4, cue reactivity was indeed established: desire to eat and salivation increased significantly over time in the cue exposure condition compared to the control condition. This was confirmed by a 2 (condition: cue exposure, 
control) x 13 (time: baseline, 1', 3', 20', 21', 23', 40', 41', 43', 60', 61', 63', 80') repeated measures ANOVA for desire to eat, in which a significant condition $\mathrm{x}$ time interaction was found, $F(12,41)=4.68, p<.001$. To examine whether extinction took place at the end of the intervention, an ANCOVA was performed to compare conditions on desire to eat at minute 80 while controlling for baseline desire to eat. Results showed that conditions did not differ anymore on desire to eat at the end of the intervention, $F(1,51)=1.03, p=.314$, indicating that extinction of the desire to eat in the exposure condition was successful. In addition, within-session habituation (WSH) was calculated, as operationalised by subtracting the end-level of cue reactivity from the peak response during the intervention (Craske et al., 2008). In line with the end level of desire to eat, WSH was significantly greater for the cue exposure condition $(M=2.15, S D=2.14)$ compared to the control condition $(M=0.70, S D$ $=1.59), t(52)=2.80, p=.007$.

The extinction of the desire to eat in the cue exposure condition was also found right after the end of the intervention, but before the meal started (BM). The 2 (condition) $\mathrm{x} 2$ (time: 80, BM) repeated measures ANOVA on desire to eat showed a significant condition $\mathrm{x}$ time interaction, $F(1,52)=11.96, p=.001$. Just before the meal started, a significant drop in desire to eat was observed in the cue exposure condition, while a steep increase in desire to eat was observed in the control condition (see Figure 3).

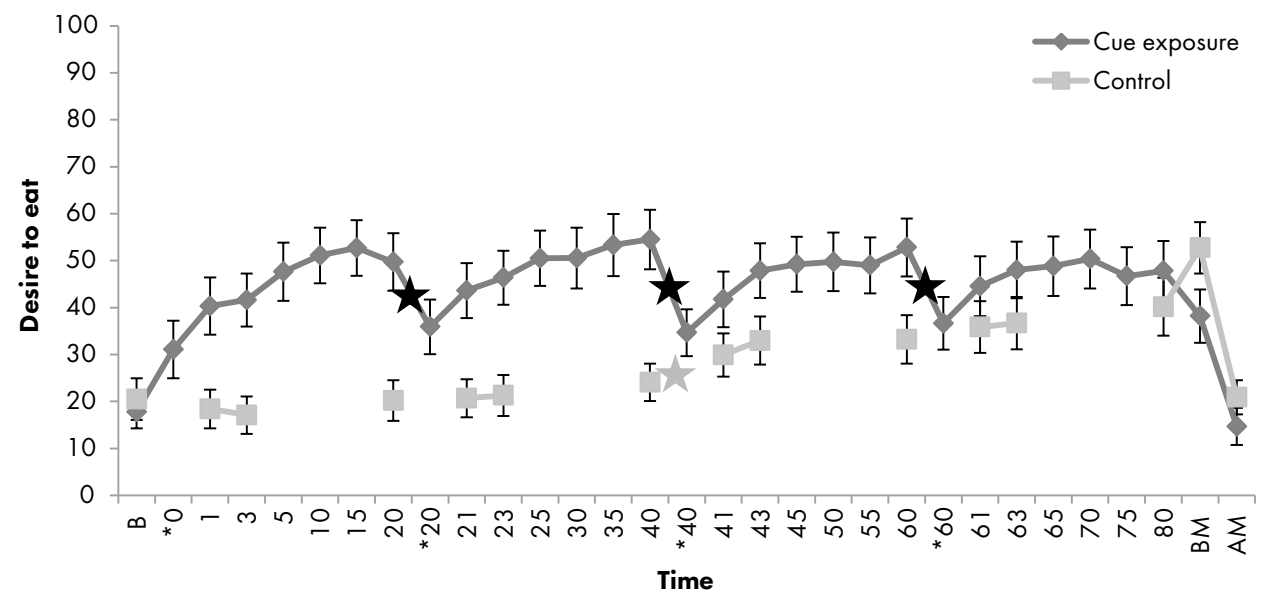

Figure 3. Mean desire to eat scores per condition (cue exposure, control) per time point at baseline (B), during the intervention (minutes $0-80$ ), and after the intervention, before the meal (BM) and after the meal (AM). Black stars represent the 10-minute breaks of the exposure condition, the grey star represents the 30-minute break of the control condition. Error bars represent standard errors of means. Additional measurements in the cue exposure condition that were taken when exposure foods were not visible are displayed with * 
The other cue reactivity measure, salivation (Figure 4), was also analysed in a $2 \times 13$ repeated measures ANOVA and showed a marginally significant condition $\mathrm{x}$ time interaction effect, $F(12,38)=1.83, p=.077$, as well as a significant main effect of condition, $F(1,49)=$ $4.38, p=.042$. Thus, though salivation responses were similar at baseline $(F<1)$, the cue exposure condition salivated significantly more than the control condition throughout the intervention. The main effect of time on salivation was not significant $(F<1)$ indicating that salivation responses remained stable over 80 minutes of intervention for both conditions. An ANCOVA on minute 80 with baseline salivation as covariate showed that participants in the exposure condition still salivated significantly more at the end of the intervention compared to the control condition, $F(1,51)=4.55, p=.038$. In line with the end level of salivation, WSH was equal for cue exposure condition $(M=0.36, S D=0.43)$ as for to the control condition $(M=0.21, S D=0.16), t(52)=1.62, p=.112$.

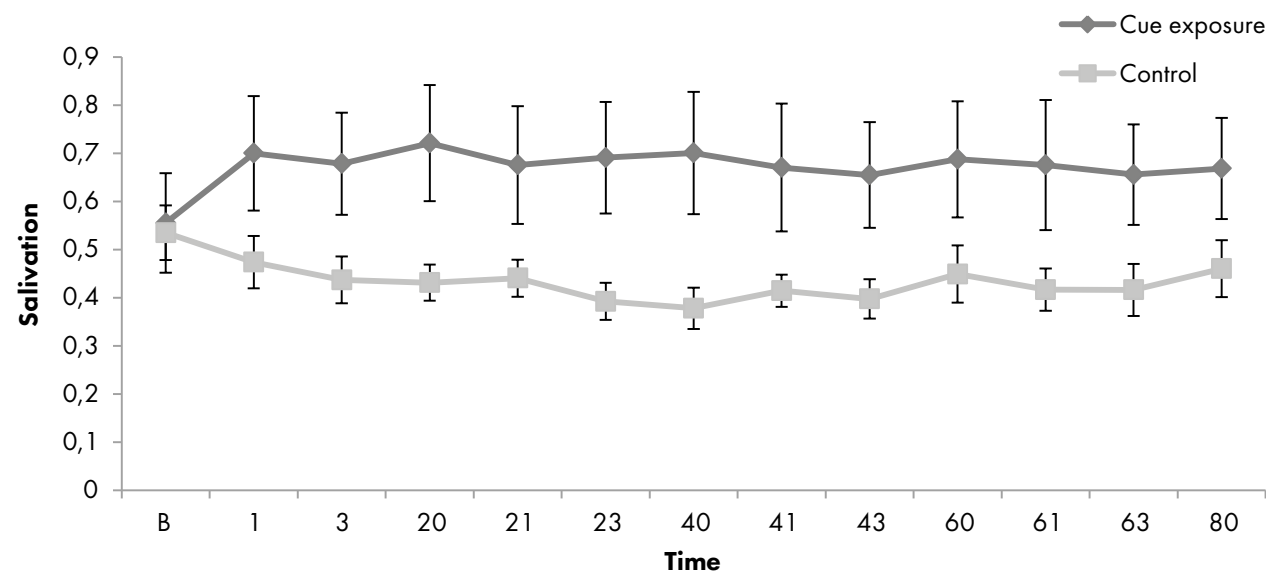

Figure 4. Mean salivation per condition (cue exposure, control) per time point at baseline (B) and during intervention (minutes $0-80$ ). Error bars represent standard errors of means.

$E A H$. All participants followed the instruction of eating a breakfast or lunch and no additional snacks before the intervention. Based on post-meal hunger ratings, six participants were excluded from the behavioural EAH analyses: five reported still being hungry after the meal (defined as score $>50 \mathrm{~mm}$ on the $100-\mathrm{mm}$ VAS), and one person had a missing value on post-meal hunger. Further, two additional participants had to be excluded from the analyses because they did not receive chocolate mousse during exposure due to a practical error, ending up with 46 participants ( $n=21$ cue exposure, $n=25$ control). Post-meal hunger ratings did not differ between conditions (cue exposure: $M=9.48, S D=$ 9.15; control: $M=11.38, S D=11.14), t(44)=0.63, p=.535$, indicating that the absence of hunger manipulation succeeded. Participants whose answers on the awareness check 
suggested they were more or less aware of the aim of the bogus taste test $(n=11)$ were equally divided over the conditions, $\chi^{2}(1,46)=0.50, p=.478$, and they did not consume less kcal in total than participants who were not at all aware, $t(44)=0.15, p=.881$, therefore these participants were not excluded from the analyses.

In order to examine to effect of cue exposure on EAH, two separate tests were performed. As chocolate mousse was an exposure item for every participant, chocolate mousse intake during EAH was analysed separately from the other food items to examine specific effects of food cue exposure. In addition, total intake during EAH (without chocolate mousse) was analysed as an indication of the generalisability of exposure effects.

Total intake. Means and SDs for consumed kcal during the bogus taste test are displayed in Table 1. Total kcal consumption (without chocolate mousse) was equal for both conditions, $t(44)=-0.71, p=.480$.

Chocolate mousse intake. The cue exposure condition consumed significantly less chocolate mousse compared to the control condition, $t(44)=-2.38, p=.022$. See Table 1 for means and SDs.

Table 1. Means and standard deviations (SD) of kcal consumed during EAH paradigm, separated by condition (cue exposure, control).

\begin{tabular}{|lcc|} 
& Cue exposure & Control \\
\cline { 2 - 3 } & $n=21$ & $n=25$ \\
\hline Total kcal $^{1}$ & $217.44(125.18)$ & $246.53(147.75)$ \\
Kcal chocolate mousse $^{2 *}$ & $22.13(17.42)$ & $39.65(31.55)$ \\
\hline
\end{tabular}

1. Total kcal intake (of all desert items excluding chocolate mousse) during taste test in EAH paradigm. 2. Kcal intake of chocolate mousse (i.e., food that was part of cue exposure intervention) during taste test in EAH paradigm. ${ }^{*} p<.05$.

Finally, correlations between intake and WSH of cue reactivity, as well as between intake and if CS then US expectancies were calculated. Regarding the cue reactivity - intake association, neither WSH of desire to eat nor WSH of salivation were significantly correlated with total kcal intake, $r(46)=-.03, p=.828$, resp. $r(46)=-.16, p=.281$, or chocolate mousse kcal intake, $r(46)=-.21, p=.167$, resp. $r(46)=-.23, p=.131$. Regarding the if CS then US' expectancy - intake association, a significant positive correlation was found between chocolate mousse intake and post-intervention 'if CS then US' expectancy, $r(46)=.34, p=$ .022 , indicating that lower CS - US expectancies were associated with less chocolate mousse intake. The correlation between 'if CS then US' expectancy and total kcal intake was not significant, $r(46)=.07, p=.665$. Thus, chocolate mousse intake was positively associated post-intervention 'if CS then US' expectancies, while chocolate mousse intake was not associated with WSH of cue reactivity. 


\section{DISCUSSION}

In the present study, food cue exposure induced, compared to a control intervention, a significant decrease of the expectancy not to be able to resist tempting foods when confronted with them, an initial increase of cue reactivity (eating desires and salivation) during the intervention, followed by extinction of eating desires at the end of the intervention, and less eating in the absence of hunger of the exposed food item (i.e. chocolate mousse). In addition, a significant positive correlation was found between post-intervention if CS then US' expectancy and chocolate mousse intake while chocolate mousse intake did not correlate with within-session habituation (WSH) of cue reactivity.

Consistent with our hypothesis, it was found that 'ifCS then US' expectancies significantly decreased during cue exposure while they did not change during the control intervention. This means that a central dysfunctional cognition associated with overeating ('If I have tasty food in front of me, then I can not resist eating it') can be changed in a single-session food cue exposure, though this cognition was not explicitly challenged during the exposure.

Recent recommendations from Craske and colleagues state that exposure is most effective when 'if CS then US' expectancies are violated (Craske et al., 2012; Craske et al., 2014). Exposure sessions should be designed in a way that US expectancies are maximally violated: strong, frequent and repeated violation of expectancies would improve and strengthen the learning of inhibitory CS - no US associations (Craske et al., 2014). For example, the length of an exposure session could be determined by the time needed for the US to be expected, instead of waiting until fear (or craving) levels have significantly decreased. In line with this, it has been found that continuing interoceptive exposure for panic disorder until US expectancies decline under $5 \%$ is more effective than standard interoceptive exposure (Deacon et al., 2013). Likewise, food cue exposure might be most effective when sessions are tailored on individual US expectancies; a session ends when the individual's belief "If I have tasty food in front of me, then I can not resist eating it" is lower than $5 \%$, instead of aiming at decreased desires to eat or other cue reactivity. In the present study, the 'if CS then US' belief significantly reduced during cue exposure but still was around $50 \%$ at the end of the exposure. It is of interest to study whether aiming more specifically at a drop of the 'if CS then US' expectancy to less than 5\%, will lead to a generalisation of the current EAH effects to other food items.

An increase in cue reactivity during the start of the intervention was found for both self-reported desire to eat and salivation but only extinction of the self-reported desire to eat was found. The final part of the intervention took place close to lunch time and all participants were explicitly told that a lunch followed the intervention. That the desire to eat at the end of the intervention did not differ anymore between conditions indicates that extinction took place, as the cue exposure participants were still intensely exposed to their 
favourite food items. Remarkable was the sudden drop in desire to eat in the cue exposure condition after the intervention: while the control condition reported an increased desire to eat just before lunch, the exposure condition reported a decrease in desire to eat. Although all participants expected a lunch, eating desires elicited by food cue exposure might be very specific: participants might have had a strong desire to eat the snack foods they were exposed to, while they did not desire to eat regular lunch foods.

Unexpectedly, extinction of the increased salivation response was not found, as mean salivation remained higher for the cue exposure condition during the entire intervention. It is possible that a salivation extinction effect needs more sessions. In a laboratory conditioning study by Van Gucht and colleagues (2008) decreased salivation was not observed in a first extinction session, while it was marginally lower in the second extinction session.

Another aim of the present study was to investigate whether a one-session cue exposure intervention decreases EAH, and whether the hypothesized decreased intake is specific for exposed food items or whether it would generalise to other food items that were not present during exposure. Indeed, the intake of the one exposure food item (chocolate mousse) was significantly lower in the exposure condition compared to the controls. Total consumption did however not significantly differ between conditions. These data show that inhibitory learning takes place during one session of food cue exposure as participants could successfully inhibit themselves when confronted with an exposed food item, though the inhibitory learning did not generalise to food items that were not present during the cue exposure. It is of interest to further study whether generalisation of inhibition learning in eating is facilitated by sleep, since fear extinction studies have shown better generalisation after a good sleep, which is explained by improved consolidation of extinction memories during sleeping (Kleim et al., 2014; Pace-Schott, Verga, Bennett, \& Spencer, 2012; Spoormaker et al., 2012; Spoormaker et al., 2010).

The correlational data show that a weaker belief in the 'If CS then US' expectancy is significantly associated with less chocolate mousse intake, while neither WSH of selfreported desires nor WSH of salivation where correlated to food intake. This finding supports the importance of inhibitory learning (if CS no US) during food cue exposure and suggests it is more important to focus on the inhibitory learning than aiming at decreased cue reactivity, which is also true for anxiety (Craske et al., 2008). Lower fear levels at the end of an exposure session do not lead to better outcomes, and even quitting exposure at the highest fear level results in equal treatment effects compared to prolonged exposures until fear declines (Rachman, Craske, Tallman, \& Solyom, 1986; Rachman, Robinson, \& Lopatka, 1987).

A limitation of the present study is the lack of long term follow-up. Although the aim of the study was to investigate within-session mechanisms, measuring outcome later in time, considering the previous point on consolidation due to sleep, might have been very 
interesting and should be considered in future studies. In addition, the used 'if CS then US' believability rating has not been used in previous research before. It is possible that this specific cognition was not as relevant for every participant, whereas personalized if CS then US' statements might better capture individual learning processes during exposure.

To conclude, the single food cue exposure session in the present study was effective in decreasing desires to eat and caloric intake of the exposed food item. Adding exposure to obesity treatment could therefore be a highly valuable component. In addition, food cue exposure also reduced 'if CS then US' expectancies, and this expectancy violation was associated with less eating of the cued food in the absence of hunger. Interestingly, habituation of the desire to eat and salivation was not at all related to intake. A clear parallel can be drawn to clinical recommendations from the anxiety literature (e.g. Craske et al., 2014): though desires to eat might be an important motivation to start exposure therapy, within-session desires should not be taken as a reference for learning. Instead, focussing on the inhibitory learning by explicitly tackling and violating verbalised CS-US expectancies during therapy might be more important for treatment success, also in overeaters and obesity.

\section{CONFLICT OF INTEREST}

The authors report no conflict of interest.

\section{ACKNOWLEDGEMENTS}

This study is part of an ongoing project that is financed by the Netherlands Organisation for Scientific Research (NWO): Vici Grant 453.10.006, awarded to Anita Jansen. The authors would like to thank Jessica Alleva for her help in composing the control intervention program and Jette Spoormans for her help in collecting data. Further, the authors would like to thank Infraligne studio Maastricht for notifying clients about the study. 




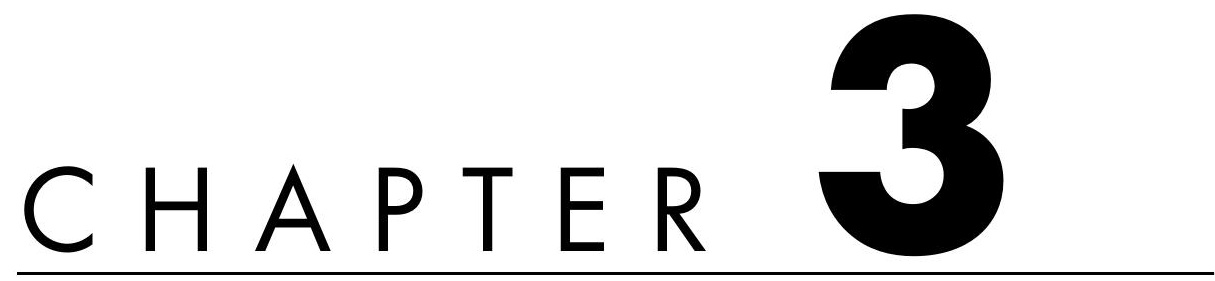

\section{CUE EXPOSURE THERAPY \\ REDUCES OVEREATING OF \\ EXPOSED AND NON-EXPOSED \\ FOODS IN OBESE ADOLESCENTS}

Published as:

Schyns, G., Roefs, A., Smulders, F. T. Y., \& Jansen, A. (2018). Cue exposure therapy reduces overeating of exposed and non-exposed foods in obese adolescents. Journal of Behavior Therapy and Experimental Psychiatry, 58, 68-77. https://doi.org/10.1016/i. jbtep.2017.09.002 


\section{ABSTRACT}

BACKGROUND AND OBJECTIVES: This study tested whether two sessions of food cue exposure therapy reduced eating in the absence of hunger $(\mathrm{EAH})$, specified for exposed and non-exposed food, in overweight and obese adolescents, and whether habituation of food cue reactivity and reduced CS-US expectancies predicted a decrease in EAH.

METHODS: 41 overweight adolescents (aged 12 - 18 years) were randomly assigned to a cue exposure intervention or a lifestyle intervention (control condition). Habituation of food cue reactivity (self-reported desire to eat and salivation) and CS-US expectancy were measured during both sessions, and EAH was measured at the end of session two.

RESULTS: Compared to the control condition, the cue exposure condition showed less EAH for the exposed food item as well as for the non-exposed food items. Larger withinsession (WSH) and between-session habituation (BSH) of cue reactivity were not related to less EAH, change in CS-US expectancy was unrelated to EAH.

LIMITATIONS: The study was underpowered, and compliance to homework instructions between sessions was poor, intervention effects might have been larger when participants adhered to daily homework exercises.

CONCLUSIONS: Food cue exposure was effective to reduce EAH of exposed and nonexposed food items, indicating generalisability of the exposure effect. In line with exposure effects in anxiety disorders, habituation was not found to benefit outcome, though the present data do also not provide evidence that CS-US expectancy violation predicts EAH.

KEYWORDS: exposure therapy, habituation, cue reactivity, obesity, expectancy violation, eating in the absence of hunger 


\section{INTRODUCTION}

Exposure-based interventions have widely received empirical support for the treatment of anxiety disorders (Deacon \& Abramowitz, 2004). In eating disorders and obesity, far less evidence is available on the effectiveness of exposure therapy, in spite of an alarming need for effective treatments. Worldwide, overweight and obesity prevalences increase disturbingly, with estimated increases of $27.5 \%$ for adults and as much as $47.1 \%$ for children falling in the overweight range between 1980 and 2013 (Ng et al., 2014). Calorie intake beyond physiological needs, also referred to as eating in the absence of hunger (EAH), is a major determinant of overweight. Several studies have reported on the significant association between EAH and adiposity in children (e.g. Butte et al., 2007; Kral et al., 2013). Triggers for EAH seem to be a core problem of overeating: increased food cue reactivity, which refers to anticipatory psychological (e.g., eating desires) and physiological responses (e.g., salivary production) that prepare for food intake, supposedly forms a major obstacle to resist palatable food and successful weight loss (A. Jansen, 1998). Indeed, a meta-analysis showed that cue-induced food cravings were prospectively related to eating and weight gain, with similar (medium) effect sizes for children and adults (Boswell \& Kober, 2016). Moreover, a pilot study showed that cue reactivity was significantly reduced in successful dieters (formerly obese) compared to unsuccessful (still obese) dieters (A. Jansen, Stegerman, Roefs, Nederkoorn, \& Havermans, 2010). These findings suggest that successfully refraining oneself from consuming high-caloric foods is related to decreased food cue reactivity, which in turn might make it easier to resist tempting foods and hence improve successful weight loss and relapse prevention.

Models of overeating state that food cue reactivity can be acquired through classical conditioning (A. Jansen, 1998): by associating food intake (unconditioned stimulus; US) with predictive cues (conditioned stimulus; CS), such as the smell and sight of food, presentations of merely the CS are capable of inducing cue reactivity (conditioned response). Results of human laboratory studies confirm that associations between food intake (US) and neutral stimuli (CS) are easily learned (Bongers, van den Akker, Havermans, \& Jansen, 2015; van den Akker, Havermans, Bouton, \& Jansen, 2014). If CS-US associations are acquired in daily life through classical conditioning, extinction of food cue reactivity, by repeated exposure to CSs without the US (eating), could be helpful to reduce overeating. During food cue exposure, participants are exposed to intake-predicting cues (CSs), like the sight of favourite food, while food intake (US) is not permitted, allowing conditioned motivation to eat to decrease over time. Pilot studies in bulimia nervosa patients show that cue exposure therapy is indeed effective in decreasing food cravings and binge eating (A. Jansen, Broekmate, \& Heymans, 1992; A. Jansen, Van den Hout, De Loof, Zandbergen, \& Griez, 1989; Martinez-mallen et al., 2007; McIntosh, Carter, Bulik, Frampton, \& Joyce, 
2011; Toro et al., 2003). With regard to overweight samples, cue exposure has shown to prevent weight regain after successful weight loss in adults (Mount, Neziroglu, \& Taylor, 1990). Interestingly, cue exposure is also effective for obese children to reduce EAH in a behavioural task (Boutelle et al., 2011, 2014). Investigating exposure therapy in children is of great importance, as obesity at a young age is not only associated with serious medical issues, it also increases the chance of being obese as an adult - along with the health consequences later in life (Serdula et al., 1993). In addition, children are less burdened with an ingrained learning history, and suggested to be more malleable than adults (e.g. faster adaptation of behavioural patterns), which make them an important target group for interventions (Wilson, 1994). Although the studies by Boutelle and colleagues (2011, 2014) found interesting result on EAH in general, it is of interest to further investigate the specificity of cue exposure effects on EAH: it has been shown in overweight adult women that a single session of cue exposure, as compared to an active control intervention, led to less EAH for the exposed foods, but no generalisation to non-exposed food items occurred (Schyns, Roefs, Mulkens, \& Jansen, 2016).

Given the limited research available, the food cue exposure domain could greatly benefit from the advances in the anxiety research field. A fascinating line of research on working mechanisms of exposure in anxiety disorders has shown that, in contrast to the original assumption that anxiety levels should habituate during therapy sessions, habituation of anxiety during (within session habituation; WSH) and between treatment sessions (between session habituation; $\mathrm{BSH}$ ) is not a good predictor of treatment outcome (Craske et al., 2008). It is now well established that extinction learning is not erasing old CS-US memories, but instead creating new CS-noUS associations; inhibitory learning (Bouton, 1993; Bouton \& King, 1983). Exposure should aim to make new CS-noUS associations as strong as possible, which can for example be achieved by violating US expectancies during exposure (Craske, Treanor, Conway, Zbozinek, \& Vervliet, 2014). Exposure for panic disorder patients aimed at violating US expectancies has shown to be more effective than aiming at habituation of fear (Salkovskis, Hackmann, Wells, Gelder, \& Clark, 2006). Strengthening inhibitory learning seems especially important for overweight and obese individuals (A. Jansen, Schyns, Bongers, \& van den Akker, 2016), as several studies found associations between overeating and obesity and weak inhibitory skills (e.g., Guerrieri, Nederkoorn, \& Jansen, 2012; Nederkoorn, Houben, Hofmann, Roefs, \& Jansen, 2010; Nederkoorn, Smulders, Havermans, Roefs, \& Jansen, 2006), also in children and adolescents (e.g., Batterink, Yokum, \& Stice, 2010; Nederkoorn, Braet, Van Eijs, Tanghe, \& Jansen, 2006). To improve inhibitory learning, the expectancy violation approach could be translated to food cue exposure by exposure to specific overeating cues that are linked to strong eating expectancies (e.g., "If I feel exhausted and palatable food is available [CS], then I will have a binge [US]") while testing whether the US indeed takes place as expected. 
Although evidence on the role of habituation and expectancy violation in cue exposure research is scarce, one study established that, in line with the findings of anxiety studies, WSH of cue reactivity (salivation and self-reported eating desires) did not predict EAH. Instead, self-reported change in CS-US expectancy was significantly related to EAH: lower US expectancies predicted less EAH of an exposed food item (Schyns et al., 2016). However, no generalisation to non-exposed foods was found. Generalisation of the exposure effect remains very important for the usability of exposure in clinical practice; it is plausible that more sessions are required for generalisation to occur, and/or better consolidation of the new CS-noUS memory, for example by sleeping after the session (Pace-Schott, Verga, Bennett, \& Spencer, 2012). Further, even when the new CS-noUS association has been wellconsolidated, the original CS-US association remains intact and forms a risk of return of responses in another context or later in time. One possible way to enhance accessibility of the CS-noUS association are retrieval cues: mental or physical cues that help to remember the extinction memory (Craske et al., 2014). Adding a retrieval cue has been shown to attenuate renewed responding after a context switch in conditioning studies (Dibbets, Havermans, \& Arntz, 2008; Vansteenwegen et al., 2006), though results in more clinically applied exposure studies are mixed (Culver, Stoyanova, \& Craske, 2011; Dibbets, Moor, \& Voncken, 2013).

The primary aim of the present experiment is to investigate whether two sessions of food cue exposure reduce EAH of the exposed food item and EAH of non-exposed food items in overweight and obese adolescents. It is hypothesised that EAH is less in the exposure condition relative to a control condition, both for exposed and non-exposed foods (generalisation). Further, the role of habituation of cue reactivity (WSH and BSH of salivation and self-reported eating desires) and the violation of US expectancies in the prediction of EAH are tested, hypothesising that WSH and BSH of habituation are positively associated with EAH if habituation is critical for extinction, whereas CS-US expectancies are related to EAH if inhibitory learning is critical. As a secondary outcome measure, the effects of cue exposure on eating psychopathology and the added benefit of a retrieval cue herein are examined at one-month follow-up.

\section{MATERIAL AND METHODS}

\section{PARTICIPANTS}

41 participants, aged 12 to 18 years were recruited from the local Area Health Authority and the paediatric obesity outpatient clinic of Zuyderland in Kerkrade, the Netherlands (see Figure 1). Participants were eligible when having sufficient Dutch speaking skills and 
being overweight according to criteria for children, as defined by scoring above the age and sex-specific BMI cut-off (BMI of 25 in adults; Cole, Bellizzi, Flegal, \& Dietz, 2000). After being informed by the researcher, children and parents received one week to consider participation. After written consent was given by the child and parents, the participant was put on a waiting list for a group of participants. As soon as six participants could make it one the same two intervention dates, the group was set by the experimenter. The condition that was assigned to each group of participants was alternated (cue exposure - lifestyle control - cue exposure, etc.). Participants had an equal chance of being randomized to one or the other condition: the dates on which the participant was available for the intervention determined in which group and thus which condition (s)he was assigned to, assignment to conditions was not based on personal characteristics, and groups were not pre-existing. Half of the groups in each condition were further randomised to a retrieval cue and no retrieval cue condition. At the moment of inclusion, neither the experimenter nor the participant had information about which group and condition the next participant would be assigned to. In addition, participants did not know between inclusion and the first session to which condition they would be assigned to; conditions were concealed until all baseline measures were completed on the first session. The study was approved by the Ethical Committee of the Faculty of Psychology and Neuroscience of Maastricht University.

\section{INTERVENTION}

\section{FOOD CUE EXPOSURE INTERVENTION}

Participants were exposed to large portions of their personalised four favourite foods that were continuously available right in front of the participant on a tray. In addition, every participant was exposed to chocolate mousse, being the standard exposure food item. Participants were coached in getting their desire to eat the food as high as possible, by smelling, touching, licking and imagining eating the food, but consumption was not allowed. During the breaks, tea towels were placed over the food trays. After the exposure in session one, one personalised food item of choice was selected for the home-work assignment, and put in a plastic container with the instruction to repeat the exposure exercise each day during the next week for at least 15 minutes. All other foods were thrown away by the participant in a garbage bin. In order to keep the homework foods appealing and unspoilt during the entire week, only non-perishable foods were selected as exposure foods.

\section{LIFESTYLE CONTROL INTERVENTION}

The lifestyle control intervention focussed on providing psycho-education about healthy eating, physical exercise, body satisfaction and weight loss. Dutch guidelines for healthy eating were provided (Voedingscentrum, 2011), and the discussed themes were regularly 
coupled with interactive exercises. Participants also received a home-work assignment, in which they were instructed to make a poster containing pictures and drawings of foods, categorised as healthy and unhealthy. The poster was presented for the other group members during the second session.

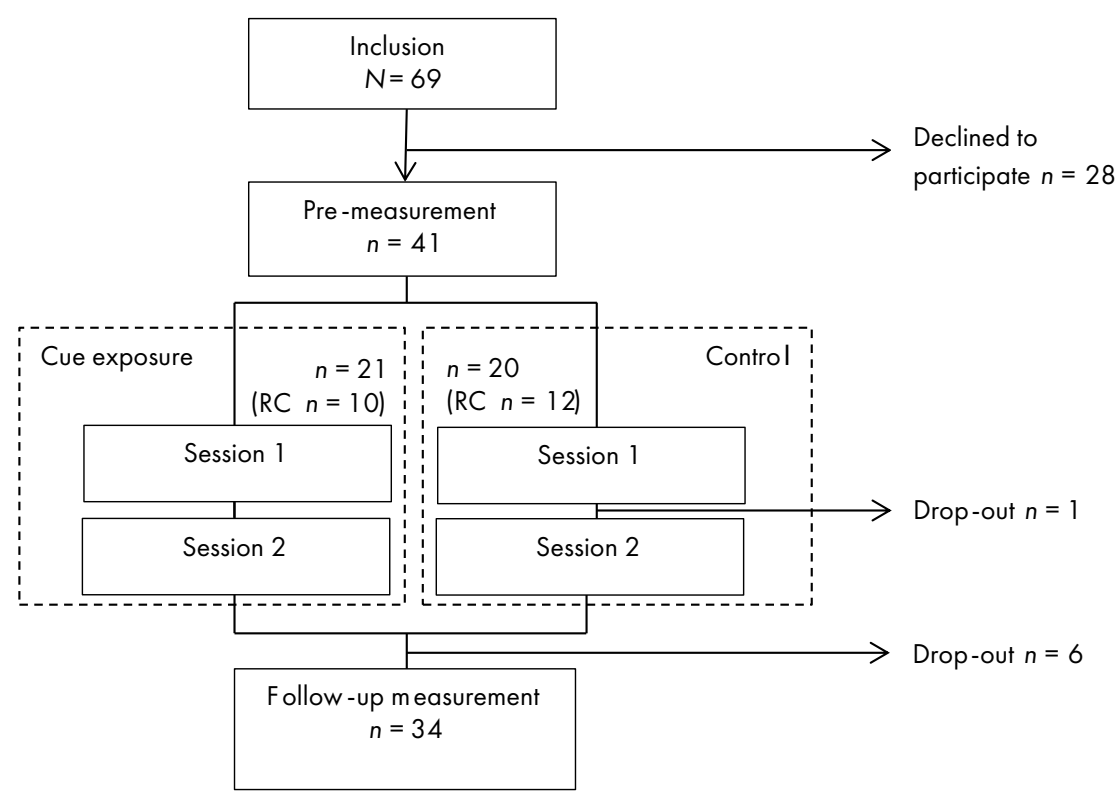

Figure 1. Inclusion, randomisation and study completion of participants. $R C=$ the number of participants within each condition that received a retrieval cue.

\section{PROCEDURE}

An overview of study measurements is displayed in Figure 2. Prior to the intervention sessions, participants filled out their personal favourite foods to determine exposure foods and the Eating Disorder Examination-Questionnaire online. In addition to the description of the favourite food, participants also rated palatability and difficulty of not eating this food. When participants were assigned to the exposure condition, the researcher selected and bought four foods that were most palatable and difficult to refrain from. After completing the online survey, participants were planned for two group sessions with exactly one week in between the two sessions. Blinding of participants and experimenters was not possible. On average, 4.1 children (range 2-7) participated in one group, and 10 groups took place in total; five of each intervention. Group sessions took place from $3 \mathrm{PM}$ to 6.45 PM, including a sandwich meal in the final part of each session, and a bogus taste test after the meal in session two. Participants were instructed to eat a regular lunch before the intervention, but 
no snacks between lunch and the intervention. Eating and drinking (except water) were not allowed during the intervention.

During each session, baseline measurements were done (saliva, CS-US expectancy and VASs on desire to eat and hunger), as well as questions on homework compliance at session two only. Then in session one, participants were given a short rationale for the intervention they were assigned to (cue exposure or lifestyle). Furthermore, participants in the retrieval cue condition received a ribbon in a colour of choice that was explicitly linked to what would be learned during the intervention, and instructed to wear the ribbon until the online follow-up measurement. They rated their expectation of the intervention, after which the intervention started. Both interventions lasted exactly 60 minutes, excluding breaks. In the cue exposure condition, there were two 10-minute breaks after every 20 minutes of exposure, while there was one 20-minute break after 40 minutes in the control condition. Saliva and desire to eat were measured at 10 time-points (at baseline and nine times during the intervention). In the cue exposure condition, 12 additional desire to eat measurements were taken throughout the intervention, including three measurements (minutes 0, 20, 40) when exposure foods were not visible. The timer was stopped for interruptions due to the measurements, resulting in 60 minutes of pure interventions. After the intervention, participants rated current hunger and CS-US expectancy. Then, all participants received the meal after which hunger was again rated. Finally, in session one, participants received information about their homework assignment, whereas in session two, the meal was followed by the bogus taste test to measure EAH. Weight and height were measured after the bogus taste test in session two.

One month after the intervention (follow-up), participants filled out the Eating Disorder Examination-Questionnaire online, as well as the questions intervention evaluation and compliance on the retrieval cue, after which they received a compensation of $€ 20$ for participation and were thanked and debriefed about the nature of the study.

\section{ASSESSMENT}

\section{EAH BEHAVIOURAL PARADIGM}

The EAH paradigm was adapted from the paradigm described by Birch and Fisher (2000) and Schyns and colleagues (2016). To induce a state of satiation, participants received a sandwich meal at the end of both sessions, and they were verbally instructed to eat until satiation. Sandwiches were freely available on a food cart, with a maximum of three sandwiches per participant. The number of consumed chicken (per sandwich 160 gr; 295.6 $\mathrm{kcal}$ ) and cheese (per sandwich $175 \mathrm{gr} ; 300.1 \mathrm{kcal}$ ) sandwiches was secretly recorded by the experimenters, in order to check whether intake before the taste test was equal for both conditions. Water and diet soda were served with the sandwiches. After finishing the 
sandwiches, participant's current hunger (2.4.5) was measured. Only after the post meal hunger rating at session two, a 10-minute bogus taste test of six desserts started. Participants were instructed to rate the taste of each of the desserts (2.4.8), while being allowed to eat as much as they wanted from the generous portions: chocolate mousse ( $\sim 80 \mathrm{~g} / \sim 142 \mathrm{kcal}$; Almhof), whipped cream ( 90g/ 165 kcal; Campina), muffin $(\sim 80 \mathrm{~g} / \sim 262 \mathrm{kcal}$; Jumbo supermarket), custard ( $190 \mathrm{~g} / \sim 173 \mathrm{kcal}$; Campina $)$, strawberry mousse $(\sim 120 \mathrm{~g} / \sim 156$ kcal; Dr. Oetker), chocolate cake ( $\sim 80 \mathrm{~g} / \sim 330 \mathrm{kcal}$; Coolmore), in total approximately 1230 $\mathrm{kcal}$. Items on the questionnaire were presented in the same order for every participant. The chocolate mousse that was used during the taste test was the same chocolate mousse as used during the exposure exercises. The taste test was done individually; tables were separated by large screens or testing occurred in different rooms. After the bogus taste test, foods were removed. Each food item was weighed before and after the test, and the number of consumed kcal was calculated. Consumed kcals for each participant, both for sandwich consumption and taste test consumption, were converted into a percentage of the participant's daily energy requirement, using the Human Energy Requirements Report (FAO/WHO/UNU, 2001). Specifically, the daily energy requirement (i.e., kcal per kg body weight per day; specific for sex and age and assuming light physical activity) was calculated per participant and the percentage of consumed kcal during the meal and taste test relative to the daily energy requirement was calculated. Regarding taste test consumption, separate percentages for exposed (i.e. chocolate mousse) vs. non-exposed food items were calculated in order to address the hypothesis on generalisation.

\section{CS-US EXPECTANCY}

Participants rated how much they believed in the following 'if CS then US' statement for overeating: 'If I have tasty food in front of me, then I can ... resist to eat it' by indicating what word was most appropriate at the present moment, ranging from 'not at all' (1) to 'very well' (5).

\section{DESIRE TO EAT}

Desire to eat was measured on a Visual Analogue Scale (VAS): 'At the present moment, I have...' ranging from 0 (no desire at all to eat tasty food) to $100 \mathrm{~mm}$ (an extreme desire to eat tasty food).

\section{SALIVATION}

Salivation was measured by placing two dental cotton rolls (Hartmann, nr2, 10x35 mm) in the mouth between the cheek and left and right lower gums. The cotton rolls stayed in the mouth for exactly one minute. They were placed and removed by the participant and kept in a sealed plastic bag that was weighed before and after the measurement on a $0.01 \mathrm{~g}$ accurate weighing scale (Mettler Toledo, PB3002). 


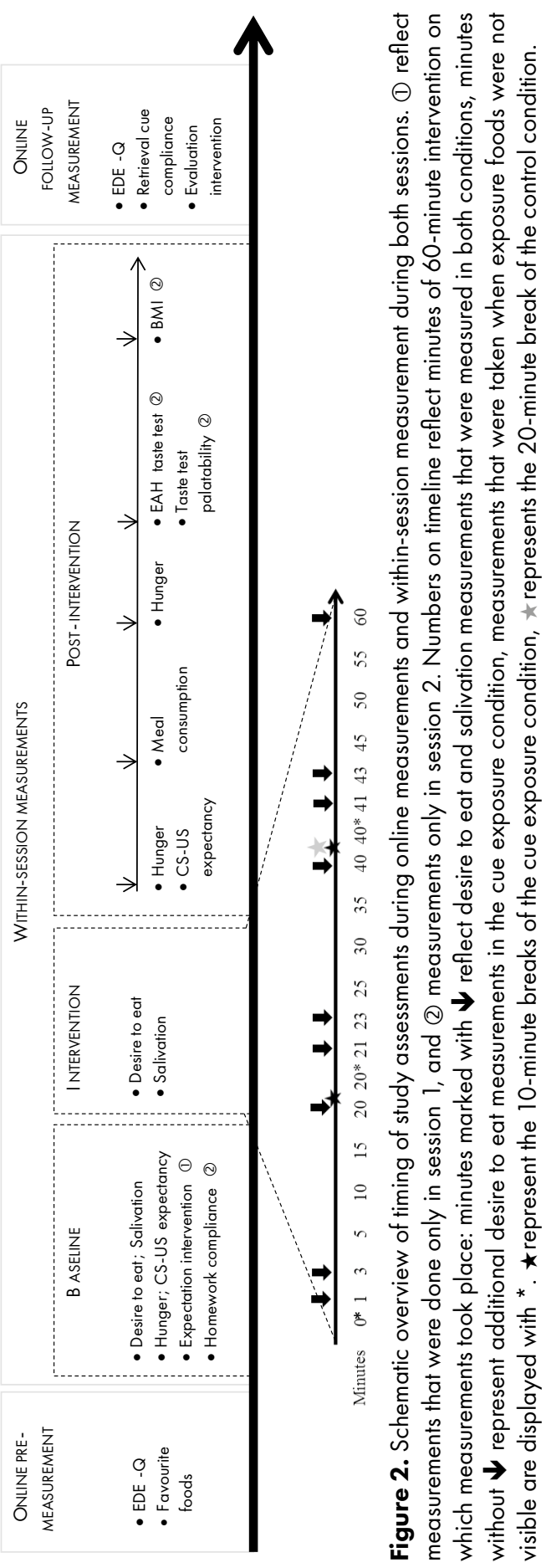




\section{HUNGER}

Current hunger was measured on a VAS: 'At the present moment, I am..., ranging from 0 (not hungry at all) to $100 \mathrm{~mm}$ (very hungry).

\section{INTERVENTION CREDIBILITY}

Each participant rated how much (s)he expected to learn / had learned from the intervention on a 5-point scale from 1 (not at all) to 5 (very much), and whether (s)he expected the intervention to help gaining control over eating, rated from 1 (much less control) to 5 (much more control).

\section{BMI}

To calculate body mass index (BMI; kg/m2), height and weight ( $0.1 \mathrm{~kg}$ accurate; My Weigh, $\mathrm{XL}-550$ ), were measured in the laboratory by the experimenter. Participants were further defined as overweight or obese using the BMI criteria for children (Cole et al., 2000).

\section{TASTE TEST PALATABILITY}

The palatability of each of the six taste test items was scored on a 0 (not palatable at all) to 100 (very palatable) VAS.

\section{EATING PSYCHOPATHOLOGY}

The Eating Disorder Examination-Questionnaire (Fairburn \& Beglin, 1994) was administered to assess eating psychopathology. The Dutch version for children was used (E. Jansen, Mulkens, Hamers, \& Jansen, 2007). 30 items target the frequency and severity of eating psychopathology, and are answered on a 7-point scale, ranging from 0 (not present) to 6 (very frequent or severe). A global score is calculated, indicating the severity of eating psychopathology, and binge eating is assessed.

\section{RETRIEVAL CUE COMPLIANCE}

To assess instruction adherence concerning the retrieval cue, a question was asked to what extent the ribbon that served as retrieval cue was worn, from 1 'not wearing the ribbon at all' to 8 'wearing the ribbon continuously'.

\section{HOMEWORK COMPLIANCE}

To check for homework compliance, three questions were asked on the number of days, amount of time and intensity to which homework exercise were performed and completed on a 5-point Likert scale, ranging from 1 (no homework done at all) to 5 (7 days/ at least 15 minutes each day/ super-intensive). 


\section{STATISTICAL ANALYSES}

Analyses on the comparison of conditions on continuous variables included independent samples t-tests, analysis of variance (ANOVA) and mixed model ANOVAs for betweensubjects and within-subjects variables, while Chi-square and Fisher's exact (in case of violation of Chi-square's assumptions) tests were used for comparisons for binary outcomes. If the Levene's test for homogeneity of variances was found to be violated, a t-test not assuming homogeneous variances was reported. The data were normally distributed. WSH and BSH were calculated for both cue reactivity measures. WSH was operationalised by subtracting the individual end-level of cue reactivity from the individual peak response during each session and averaging the scores for the two sessions. BSH was calculated by subtracting the individual peak response from session two from the individual peak response of session one (Craske et al., 2008). Mediational effects of habituation (condition $\rightarrow$ habituation [mediator] $\rightarrow$ EAH) were tested by applying the bootstrapping method with a 95\% confidence interval of the indirect effect using 5000 samples as described by Preacher and Hayes (2008). Indirect mediation effects are considered significant when the $95 \%$ confidence interval does not contain zero. Per-protocol analyses were applied to investigate the true effects and working mechanisms of the exposure intervention for participants who completed both exposure sessions. No data were estimated for participants $(n=6)$ who were lost to follow-up after the intervention, as applying the recommended last value carried forward strategy (Shah, 2011) would lead to a serious underestimation of the time effect, because the only available EDE-Q data are pre-treatment scores. To correct for the possibility of type I errors for two primary outcomes (i.e., intake of exposed and nonexposed foods) and two secondary outcomes (i.e., EDE-Q global score and binge eating), obtained p-values were adjusted for multiple comparisons using the sequentially rejective Bonferroni-Holm correction (Holm, 1979).

\section{RESULTS}

\section{DESCRIPTIVES}

As presented in Table 1, (unadjusted) BMI, age, weight status, gender and binge eating status did not differ between the two conditions. 


\section{CREDIBILITY AND COMPLIANCE TO HOMEWORK AND RETRIEVAL CUE INSTRUCTIONS}

As displayed in Table 1, the mean interventions' expectations and evaluations were equal for both conditions. Retrieval cue compliance was poor; only four of 22 participants kept the ribbon on until the follow-up measurement, data on retrieval cue effects were therefore not further analysed. Compliance of homework assignments was poor as well: while participants in the cue exposure condition where instructed to do the exposure assignment each day, only one of 21 participants followed this instruction, and four participants practiced five to six days. The mean number of days, intensity and minutes spent on homework were not-significantly different between conditions, as can be seen in Table 1. Although not significant, the mean number of days spent on homework were marginally higher in the cue exposure condition vs control condition.

Table 1. Comparison baseline characteristics, intervention credibility and homework compliance scores between the cue exposure and control condition

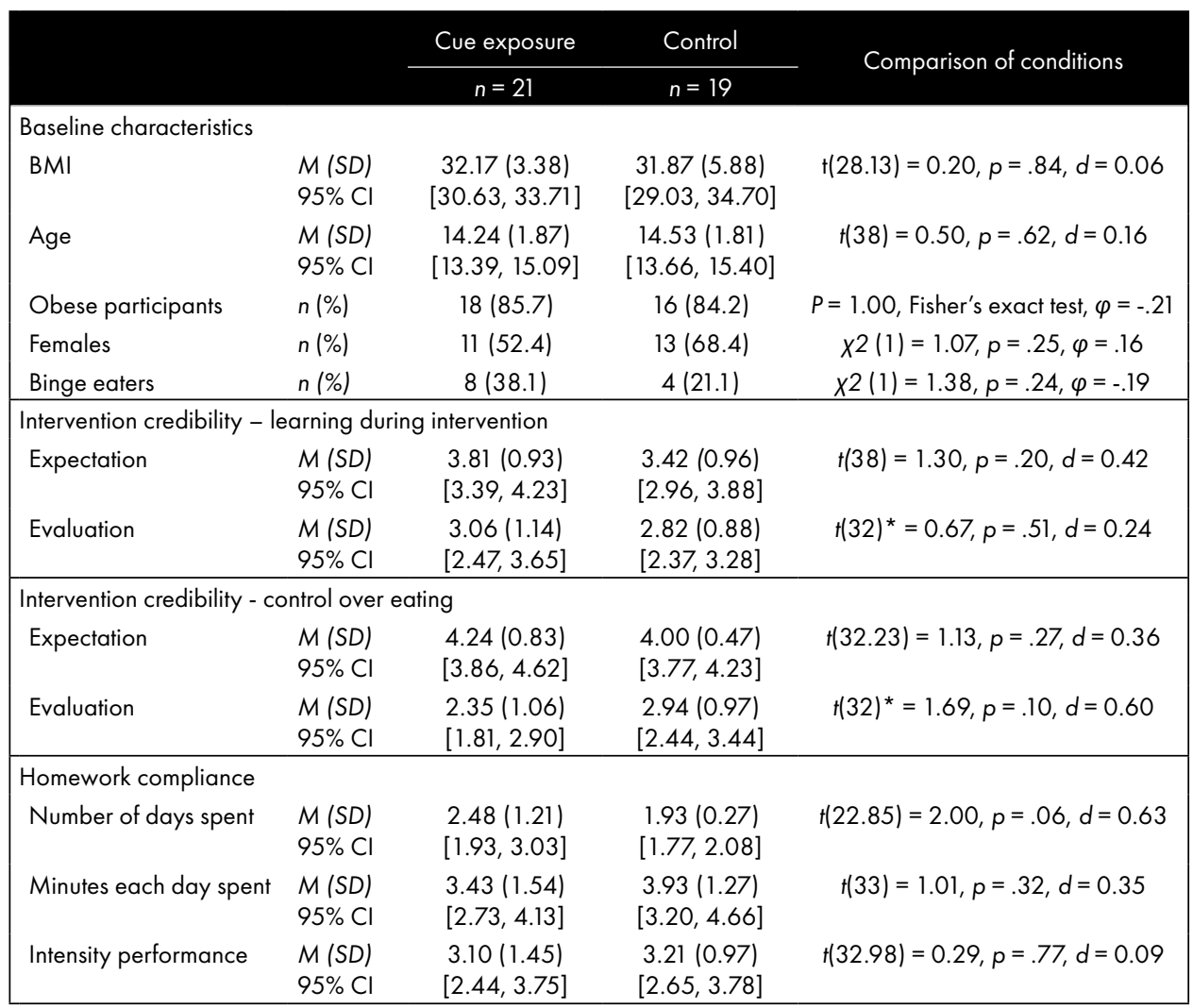

*Degrees of freedom are different for this measure, due to drop-out at follow-up. $M=$ mean; $S D=$ standard deviation; $\mathrm{Cl}=$ confidence interval. 


\section{EAH}

\section{HUNGER}

As shown in Table 2, participants in both conditions experienced an equal increase in hunger during the intervention in both sessions, followed by an equal decrease in hunger after meal consumption. This was confirmed by significant main effects of time in a 2 (condition: cue exposure, control) $\times 3$ (time: baseline, pre-meal, post-meal) mixed model ANOVA for session one, $F(2,37)=57.99, p<.001, \eta_{p}{ }^{2}=.76$, and session two, $F(2,37)=$ $30.18, p<.001, \eta_{p}^{2}=.62$. The effects of the time $\times$ condition interactions were not significant for either session one, $F(2,37)=0.13, p=.878, \eta_{p}^{2}=.01$, nor session two, $F(2,37)=1.25$, $p=.299, \eta_{p}^{2}=.06$. No significant main effect of condition was found for session one, $F(1$, $38)=0.09, p=.762, \eta_{p}^{2}<.01$, or session two, $F(1,38)=3.76, p=.060, \eta_{p}^{2}=.09$, although the mean hunger rating over the three time-points on session two were marginally higher in the control condition than the cue exposure condition. However, post-meal hunger ratings before starting the taste test in session two were not significantly different between both conditions, $t(38)=1.61, p=.116, d=0.51$. All participants reported not being hungry anymore after the meal in session two (all hunger scores $<25$ on a 100 scale), indicating that the absence of hunger manipulation was successful and not significantly different between both conditions before starting the EAH taste test.

Table 2. Means (M), standard deviations (SD) and $95 \%$ confidence intervals $(\mathrm{CI})$ of hunger ratings at baseline, before and after meal consumption in each session, separated by condition (cue exposure, control)

\begin{tabular}{|c|c|c|c|c|c|c|}
\hline & \multicolumn{3}{|c|}{ Session 1} & \multicolumn{3}{|c|}{ Session 2} \\
\hline & Baseline & Pre-meal & Post-meal & Baseline & Pre-meal & Post-meal \\
\hline \multicolumn{7}{|c|}{ Cue exposure $(n=21)$} \\
\hline$M(S D)$ & $18.24(18.59)$ & $47.86(27.75)$ & $5.95(11.62)$ & $10.05(12.22)$ & $32.55(30.05)$ & 3.19 (4.99) \\
\hline $95 \% \mathrm{Cl}$ & {$[9.78,26.70]$} & {$[35.23,60.49]$} & {$[0.66,11.24]$} & {$[4.48,15.61]$} & {$[18.87,46.23]$} & {$[0.92,5,46]$} \\
\hline \multicolumn{7}{|c|}{ Control $(n=19)$} \\
\hline$M(S D)$ & $20.08(20.74)$ & $50.63(24.03)$ & $5.47(7.72)$ & $21.40(26.42)$ & $48.03(31.63)$ & $6.24(6.90)$ \\
\hline $95 \% \mathrm{Cl}$ & {$[10.08,30.07]$} & {$[39.05,62,21]$} & {$[1.75,9.20]$} & {$[8.66,34.13]$} & {$[32.78,63.27]$} & {$[2.91,9.56]$} \\
\hline
\end{tabular}

\section{MEAL}

Average energy intake of sandwiches was $726.33 \mathrm{kcal}(S D=344.40)$ in the first session and $652.63 \mathrm{kcal}(S D=256.38)$ in the second session. The percentage of energy intake during the meal relative to the individually daily required amount of energy did not differ significantly between conditions, for both session one (cue exposure: $M=18.84, S D=6.30$; control: $M$ $=20.59, S D=12.51 ; t(38)=0.55, p=.588, d=0.18$ ), and session two (cue exposure: $M=$ $17.36, S D=5.59$; control: $M=18.52, S D=10.06 ; t(38)=0.44, p=.661, d=0.14)$. 


\section{TASTE TEST}

Average palatability ratings of the taste test items did not differ significantly between conditions, neither for the exposed food item chocolate mousse $t(38)=0.56, p=.580, d=$ 0.18 , nor for the non-exposed food items, $t(38)=1.26, p=.215, d=0.41$. Further, to test the influence of hunger before the taste test on $\mathrm{EAH}$, hunger was included as a covariate in an ANOVA with percentage intake of all foods (exposed and non-exposed) as dependent variable and condition as fixed factor. The results of this analysis showed that condition was a significant predictor of food intake, $F(1,37)=6.08, p=.018, \eta_{p}{ }^{2}=.141$, while hunger in included in the model did not have a significant influence on intake, $F(1,37)=1.21, p=$ $.279, \eta_{p}^{2}=.032$, suggesting that hunger did not play a role in differences in percentage food intake below (3.3.3.1. and 3.3.3.2).

\section{EXPOSED FOOD: CHOCOLATE MOUSSE}

As presented in Table 3, in line with the hypothesis, the percentage exposure food item consumption (i.e., chocolate mousse) relative to the daily required energy was significantly lower in the cue exposure condition than in the control condition, $t(25.32)=2.52, p=.018$, $d=0.80$.

\section{NON-EXPOSED FOOD ITEMS}

Also in line with the hypothesis, the percentage non-exposed food items consumption relative to the daily required energy was also significantly lower in the cue exposure condition than in the control condition, $t(28.58)=2.32, p=.028, d=0.76$, see Table 3 .

Both effects of condition on intake of exposed and non-exposed foods remained significant according to the sequentially rejective Bonferroni-Holm correction (Holm, 1979).

Table 3. Means $(M)$, standard deviations (SD) and $95 \%$ confidence intervals $(\mathrm{Cl})$ of absolute kcal consumption and percentage of consumed kcal relative to the individual daily amount of required energy during the EAH paradigm, separated by condition (cue exposure, control)

\begin{tabular}{|lcccc|}
\hline & \multicolumn{2}{c}{ Exposed food item ${ }^{1}$} & \multicolumn{2}{c|}{ Non-exposed food items $^{2}$} \\
\cline { 2 - 5 } & Absolute kcal & $\begin{array}{c}\text { Percentage of daily } \\
\text { required kcal }{ }^{3}\end{array}$ & $\begin{array}{c}\text { Absolute kcal } \\
\text { Cue exposure }(n=21)\end{array}$ & $\begin{array}{c}\text { Percentage of daily } \\
\text { required kcal }^{3}\end{array}$ \\
$\mathrm{M}(\mathrm{SD})$ & $23.05(28.24)$ & $0.57(0.68)$ & $257.68(184.39)$ & $6.37(4.34)$ \\
$95 \% \mathrm{Cl}$ & {$[10.19,35.90]$} & {$[0.260 .88]$} & {$[173.74,341,61]$} & {$[4.39,8,35]$} \\
\hline Control $(n=19)$ & $47.40(43.72)$ & $1.46(1.41)$ & $357.72(232.55)$ & $10.86(7.36)$ \\
$M(S D)$ & {$[26.33,68.47]$} & {$[0.78,2.15]$} & {$[245.63,469.80]$} & {$[7.32,14.41]$} \\
\hline $95 \% \mathrm{Cl}$ & & & & \\
\hline
\end{tabular}

1. Kcal intake of the item that was used during cue exposure: chocolate mousse. 2. Total kcal intake of items not used during cue exposure (whipped cream, muffin, custard, strawberry mousse, chocolate cake). 3. Percentage eaten of individual daily required amount of energy, adjusted for sex, age and weight (FAO/WHO/UNU, 2001) 


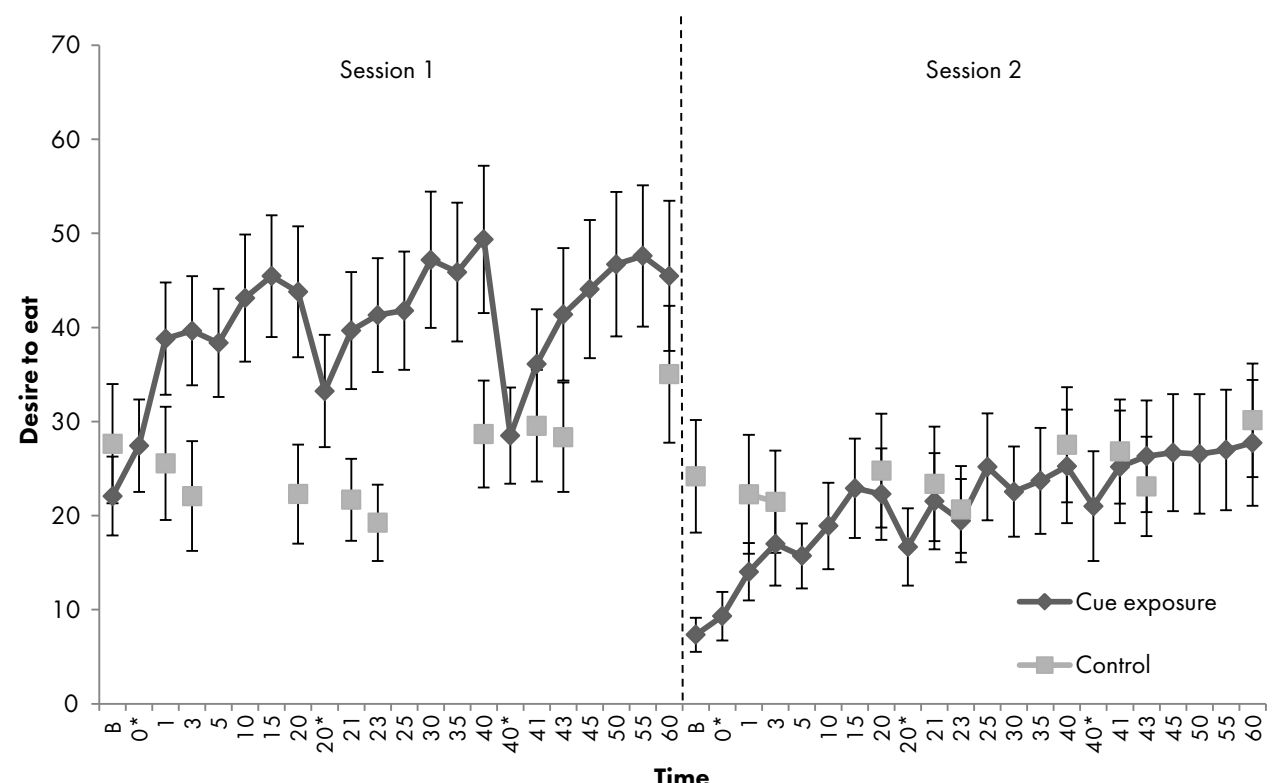

Figure 3. Mean desire to eat scores per condition (cue exposure, control) per time point at baseline (B) and during the intervention (minutes $0-60$ ) on session one and two. Error bars represent standard errors of means. Additional measurements in the cue exposure condition that were taken when exposure foods were not visible are displayed with *.

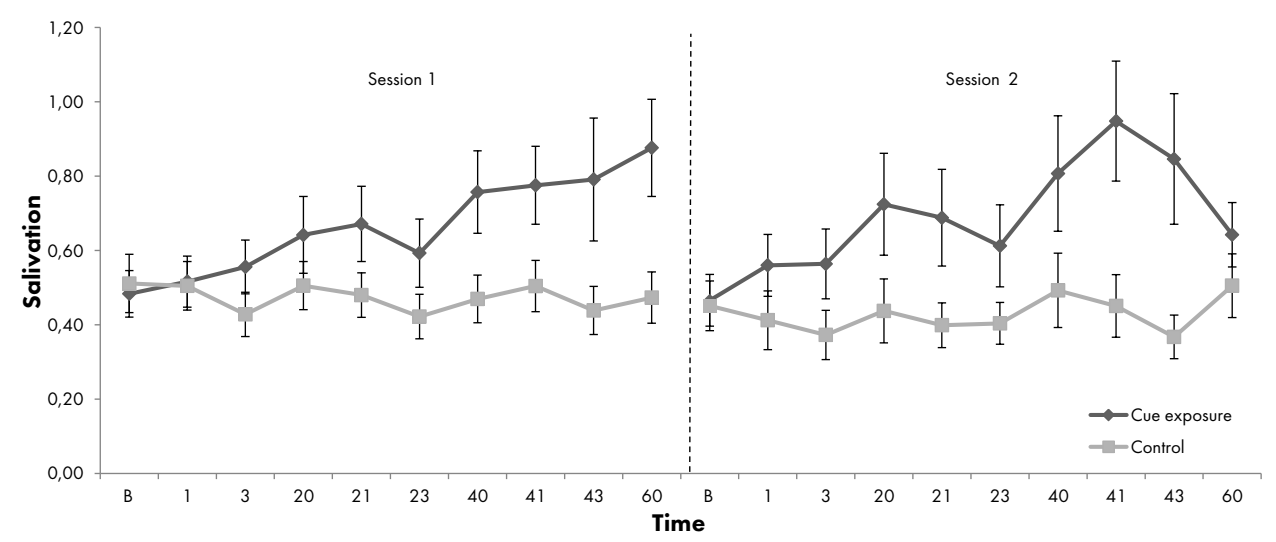

Figure 4. Mean salivation per condition (cue exposure, control) per time point at baseline (B) and during the intervention (minutes $0-60$ ) on session one and two. Error bars represent standard errors of means. 


\section{PREDICTORS OF EAH}

\section{HABITUATION OF CUE REACTIVITY}

Figures 3 and 4 show the mean patterns of cue reactivity, desire to eat and salivation, during both intervention sessions per condition. Results of the mediation analyses suggest that neither WSH (desire to eat: $\beta<-.01,95 \%$ CI $[-.05, .06]$; salivation: $\beta=.02,95 \%$ CI $[-.04$, $.14]$ ), nor BSH (desire to eat: $\beta=-.05,95 \%$ CI $[-.27, .13]$; salivation: $\beta<-.01,95 \%$ CI $[-.05$, $.06)$ were significant mediators between condition and EAH of the exposed food item. Regarding EAH of non-exposed food items, the indirect effect between condition and EAH via WSH of desire to eat did not include zero, $\beta=-1.01,95 \%$ CI $[-2.65,-0.04]$, indicating that the amount of WSH of desire to eat mediated the relationship between condition and EAH.

In this model, condition was significantly associated with EAH (c-path, consistent with 3.3.3.2; $\beta=4.49, t(38)=2.38, p=.022)$, the relationship between condition and habituation (a-path) was not significant, $\beta=-0.77, t(38)=1.57, p=.124$, indicating that WSH of desire to eat was not significantly different between conditions, while the relationship between habituation and EAH (b-path) of this model was statistically significant with a positive coefficient, $\beta=1.37, t(38)=2.32, p=.026$, indicating that more WSH of desire to eat was related to more EAH of the non-exposed food items. The other habituation measures were not found to be significant mediators in the relationship between condition and EAH of the non-exposed food items (BSH of desire to eat: $\beta=0.53,95 \%$ CI [-0.60, 2.57]; WSH of salivation: $\beta=-0.69,95 \% \mathrm{CI}[-2.31,0.18]$; BSH of salivation: $\beta=-0.21,95 \% \mathrm{CI}[-1.12,0.28])$.

\section{CS-US EXPECTANCY VIOLATION}

CS-US expectancy change scores were calculated (baseline session one minus postintervention session two). Due to practical errors, the CS-US expectancies were not assessed in eight participants (cue exposure $n=3$; $\operatorname{control} n=5$ ). As the assumptions on expected cell frequencies of the Chi-square test were violated, CS-US expectancy change scores were merged into two categories: expectancy violation (change score $\geq 1$ ) vs no expectancy violation (change score $\leq 0$ ). No significant differences were found between conditions regarding expectancy violation, $\chi^{2}(1, n=32)=3.35, p=.067, \varphi=-.32$, although the proportion of individuals experiencing expectancy violations was marginally higher in the cue exposure condition (11 of 18 participants) compared to the control condition (four of 14 participants). With regard to EAH, no significant interactions were found in a 2 (condition) $\times 2$ (expectancy violation: yes vs no) ANOVA for the exposed food item, $F(1,28)=0.06, p=.814, \eta_{p}^{2}<.01$, and non-exposed food items $F(1,28)=0.27, p=.605, \eta_{p}^{2}$ $=.01$, nor were any main effects found of expectancy violation on EAH of the exposed food item, $F(1,30)=0.17, p=.680, \eta_{p}{ }^{2}<.01$, or non-exposed food items, $F(1,30)=1.06, p=.311$, $\eta_{p}^{2}=.03$. 


\section{EATING PSYCHOPATHOLOGY}

The Eating Disorder Examination-Questionnaire global score decreased significantly from pre-measurement (cue exposure: $M=2.35, S D=1.17$; control: $M=2.21, S D=0.98$ ) to follow-up (cue exposure: $M=2.03, S D=1.14$; control: $M=1.91, S D=0.97$ ) in both conditions, as reflected by a significant main effect of time in a 2 (condition) $\times 2$ (time: pre vs. follow-up measurement) mixed model ANOVA, $F(1,32)=5.26, p=.029, \eta_{p}^{2}=.14$, and no significant condition $\times$ time interaction $F(1,32)=0.11, p=.744, \eta_{p}{ }^{2}<.01$. No main effect of condition was found, $F(1,32)=0.11, p=.744, \eta_{p}^{2}<.01$. At follow-up, four participants still reported binge eating relative to 12 participants at pre-measurement, the change of binge eating status was not significantly different between conditions, $P=.398$, Fisher's exact test. Results remain unchanged when using the sequentially rejective BonferroniHolm correction (Holm, 1979).

\section{DISCUSSION}

The main aim of the present study was two-fold: (1) to investigate whether two sessions of cue exposure lead to less EAH of the exposed food item and less EAH of non-exposed food items (generalisation), and (2) to investigate the predictive value of cue reactivity habituation and CS-US expectancy violation for EAH. Consistent with the a-priori hypothesis, it was found that cue exposure led to less EAH of the exposed and non-exposed food items, relative to the control condition. Habituation of cue reactivity either within or between sessions did not mediate the relationship between condition and EAH, except for WSH of desire to eat. WSH of desire to eat was found to be a significant mediator, but unbeneficial for treatment outcome: larger habituation was associated with more EAH. Unexpectedly, CS-US expectancy violation was not predictive of EAH. With regard to the secondary outcome measure, eating psychopathology improved over time, but not significantly different between conditions.

The finding of less EAH of the exposed food item after two exposure sessions in the present study replicates the finding that was previously found with one exposure session in overweight adult women (Schyns et al., 2016). However, no significant differences were found in the one-session study on EAH of the non-exposed food items whereas a difference was found in the current two-session study with a younger sample. The current finding of less EAH of non-exposed food items indicates generalisability of the exposure effect: the exposure was not only effective for the items that were present during the exposure session, the learning transferred to non-exposed highly palatable foods. This could potentially be an important notion for clinical practice; the current data might indicate that doing multiple exposure sessions makes it not essential to include all 'problematic' foods during 
exposure sessions. Moreover, the significant differences in EAH of non-exposed food items of approximately $100 \mathrm{kcal}$ during one meal are not only statistically significant, but also clinically relevant for weight loss, indicating the need to study long-term exposure effects on weight loss in the future. Generalisation of the exposure effect does however not automatically imply that randomly selected palatable foods can be used for exposure sessions, it seems plausible to use individually selected favourite foods in order to maximise learning experiences during exposure sessions.

The differences on EAH between both conditions could not be explained by potentially differential hunger levels between conditions before starting the taste test. The finding of generalisation in the present study where participants had sufficient time to consolidate the new CS-noUS memory fits well with the findings of Pace-Schott et al. (2012), who suggest that sleeping between sessions improves generalisation of exposure effects. An alternative, or additive, explanation for finding generalisation in the present study could be the sample of adolescents who might be better learners than adults and therefore constitute a good target group for interventions. Note that these explanations are speculative, as we did not manipulate sleep or selectively study age within the present experiment.

It was further studied whether habituation of food cue reactivity and expectancy violation served as predictors of EAH. Regarding cue reactivity, neither habituation within exposure sessions (WSH) or between sessions (BSH), for both self-reported eating desires and salivation, mediated the relationship between condition and EAH, except for WSH of desire to eat. WSH of desire to eat was a significant mediator between condition and EAH of non-exposed food items. However, further inspection indicated that larger WSH was associated with more $\mathrm{EAH}$, suggesting that aiming at increasing habituation of eating desires during exposure might have a negative impact. However, this could be a coincidental finding and needs to be replicated. From the current results, it can be concluded that habituation of cue reactivity, including salivation and eating desires, did not have beneficial effects on EAH, which is in line with previous findings (Schyns et al., 2016). Moreover, these findings indicate that the non-promoting role of habituation for exposure therapy outcome in anxiety disorders (Craske et al., 2008) might also hold for food cue exposure therapy. As suggested in the anxiety literature, exposure should aim at increasing associative strength of the inhibitory CS-noUS association, for example by violating CS-US expectancies (Craske et al., 2014). However, current data did not provide support for the importance of CS-US expectancy violation for EAH, which contrasts previous research (Schyns et al., 2016). Our findings suggest that expectancy violation does not play an important role in overeating, which is in line with a recent conditioning study in which expectancy violation did not lead to lower conditioned desires at test compared to a control condition (van den Akker, van den Broek, Havermans, \& Jansen, 2016). Alternatively, finding no relationship between expectancy violation and EAH can be explained by the fact that the exposure 
sessions were not designed to explicitly tackle CS-US expectancies, instead a standard exposure procedure was used that might not have been sufficient to violate expectancies in all participants. More research on the importance of expectancy violation for food cue exposure therapy outcomes is clearly warranted. An interesting and challenging next step would be to manipulate expectancy violation during cue exposure sessions.

Looking at eating disorder psychopathology one month after the intervention, both conditions improved significantly on the Eating Disorder Examination-Questionnaire global score; cue exposure was not superior in this respect. No changes were found for binge eating. The effects of retrieval cues on eating psychopathology could not be studied due to poor compliance to the use of the retrieval cue and posits a challenge for studies and clinical practice to effectively apply exposure in adolescents, for example by including parents to facilitate adherence to all intervention components (including retrieval cues and homework assignments).

It should be noted that the present study was underpowered. A larger effect size was expected for kcal intake of exposed food in the current study compared to a recent singlesession cue exposure study (Schyns et al., 2016), as adolescent participants were expected to be faster learners, and participants received two sessions of cue exposure instead of one session. Although no a priori power analysis was conducted, a post hoc power analysis indicates that 68 participants would have been required in total ( $n=34$ per condition) to detect an effect size of .80 with the desired power of $90 \%$ and alpha of .05 . Because 40 participants were included, interpretations of the study results should be done with caution, and replication of the study effects is necessary. Further, the CS-US expectancy item has not been validated and might not have been applicable to every single participant to capture individual learning processes. Also, adherence to the homework instructions during the week between both sessions was poor; intervention effects might have even been larger when participants would have done daily homework exercises. Finally, it should be noted that a taste test was not included at pre-test to prevent pre-test sensitization (i.e., allowing participants to formulate hypotheses about the study's goals and realize what is being measured in the taste test, potentially causing them to respond differently to the treatment and at post-test; Bordens \& Abbott, 2011). Because participants were randomized to one of either conditions, it is justified and sufficient to interpret post-intervention EAH scores (Gruijters, 2016). 


\section{CONCLUSIONS}

Food cue exposure was effective to reduce EAH of exposed and non-exposed food items, indicating that exposure not only works for foods that were present during exposure sessions, but that inhibitory learning transfers to other foods as well. Consistent with exposure research in anxiety disorders, habituation within and between sessions was not found consistently related, or in any case not beneficial, to EAH: though eating desires might be an important motivation to start cue exposure therapy, the present data suggest that habituation of eating desires is not necessary during exposure sessions. However, the present data also suggest that expectancy violation is not necessary for reducing EAH. More research is clearly needed to understand the working mechanisms in cue exposure therapy for overeating and obesity.

\section{CONFLICT OF INTEREST}

The authors report no conflict of interest. 



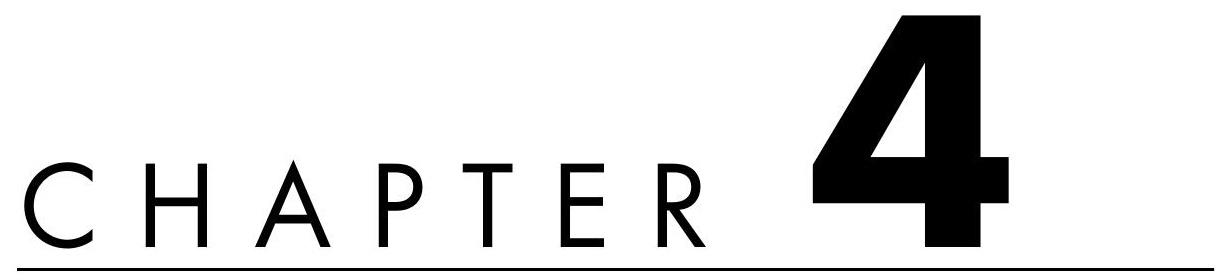

\section{ENHANCING INHIBITORY \\ LEARNING TO REDUCE \\ OVEREATING: DESIGN \\ AND RATIONALE OF A CUE \\ EXPOSURE THERAPY TRIAL \\ IN OVERWEIGHT AND \\ OBESE WOMEN}

\section{Published as:}

Schyns, G. ${ }^{*}$, van den Akker, K. ${ }^{*}$, \& Jansen, A. (2016). Enhancing inhibitory learning to reduce overeating: Design and rationale of a cue exposure therapy trial in overweight and obese women. Contemporary Clinical Trials, 49, 85-91. https://doi.org/10.1016/j. cct.2016.06.008

* The first two authors equally contributed to the manuscript. 


\section{ABSTRACT}

The prevalence of overweight and obesity has increased substantially over the last decades. Weight loss attempts in overweight individuals are common, though they seldom result in successful long-term weight loss. One very promising treatment is food cue exposure therapy, during which overweight individuals are repeatedly exposed to food-associated cues (e.g., the sight, smell and taste of high-calorie foods, overeating environments) without eating in order to extinguish cue-elicited appetitive responses to food cues. However, only few studies have tested the effectiveness of cue exposure, especially with regards to weight loss. For exposure treatment of anxiety disorders, it has been proposed that inhibitory learning is critical for exposure to be effective. In this RCT, we translated techniques proposed by Craske, Treanor, Conway, Zbozinek, and Vervliet (2014) to the appetitive domain and developed a novel cue exposure therapy for overeating aimed at maximizing inhibitory learning. The current RCT tested the effectiveness of this 8-session cue exposure intervention relative to a control intervention in 45 overweight adult (aged 18-60) females at post-treatment and 3-month follow-up, of which 39 participants completed the study. Weight loss, eating psychopathology, food cue reactivity, and snacking behaviour were studied as main treatment outcomes, and mediators and moderators of treatment effects were studied. The presented study design represents an innovative effort to provide valuable clinical recommendations for the treatment of overeating and obesity.

KEYWORDS: exposure therapy, inhibitory learning, expectancy violation, obesity, weight loss 


\section{INTRODUCTION}

A large proportion of overweight and obese individuals engage in weight loss attempts (Bish et al., 2005). However, successful long-term weight loss seems very difficult to achieve: it has been estimated that only $20 \%$ of overweight individuals are successful at losing at least $10 \%$ of their initial weight and maintaining this loss for at least one year (Wing \& Phelan, 2005). A major cause of eating more than physiologically needed is thought to be an increased reactivity to food cues. This food cue reactivity includes anticipatory psychological (i.e., craving) and physiological (e.g., increased salivation) responses that prepare an organism for food intake and promote (over)eating (Boswell \& Kober, 2016). Overweight individuals and binge eaters show greater reactivity to food cues (e.g., Ferriday \& Brunstrom, 2011; Vögele \& Florin, 1997), while reactivity seems to be reduced in successful weight loss maintainers (Jansen, Stegerman, Roefs, Nederkoorn, \& Havermans, 2010). This suggests that aiming to diminish food cue reactivity during treatment might effectively reduce overeating and promote weight loss.

Food cue reactivity is at least partly learned (e.g., Jansen, 1998): food cues (conditioned stimuli or CSs; e.g., the smell and sight of food) have become associated with eating (unconditioned stimulus or US) through repeated pairings, and these CSs can subsequently elicit cue reactivity. Similarly, learning models predict that extinction of cue reactivity can be achieved through repeated exposure to CSs without the US (eating), thereby lowering cue-elicited motivation to eat (e.g., Jansen, Havermans, \& Nederkoorn, 2011; van den Akker, Havermans, Bouton, \& Jansen, 2014). This is the aim of cue exposure therapy, in which individuals with overweight and/or eating disorders are repeatedly exposed to their personal food cues without eating. Studies on cue exposure therapy are scarce, though the limited findings indeed show substantial reductions in cue-elicited cravings and eating binges (e.g., Boutelle et al., 2014; Jansen, Broekmate, \& Heymans, 1992; Jansen, Van den Hout, De Loof, Zandbergen, \& Griez, 1989; Martinez-mallen et al., 2007; Schyns, Roefs, Mulkens, \& Jansen, 2016; Toro et al., 2003). However, whether cue exposure is also effective in facilitating weight loss remains unclear. Moreover, although a long-term abstinence of binge eating has been found (Jansen et al., 1992; Toro et al., 2003), returns of cravings and overeating at follow-up have also been reported (Boutelle et al., 2014), suggesting that cue exposure therapy might require optimization in order to have long-lasting effects.

The finding that appetitive responses can return after treatment is line with the idea that during extinction, the original CS-US association is not destroyed. Rather, a new association is formed: CS means no US (Bouton, 1993; Bouton \& King, 1983). This inhibitory CS-noUS association is relatively fragile and context-dependent, which is why responses can return even when they have been successfully extinguished. Hence, strengthening inhibitory CSnoUS associations in cue exposure therapy may help improve long-term outcome. For the 
treatment of anxiety disorders, Craske and colleagues (Craske, Treanor, Conway, Zbozinek, \& Vervliet, 2014) have recently proposed a number of exposure techniques to achieve this. One example is to design exposure sessions in a way that maximizes the violation of CSUS expectancies - if the non-occurrence of the US is surprising, this should strengthen (inhibitory) learning. In cue exposure, this may be achieved by identifying a patient's specific overeating cues that are linked to strong eating expectancies [e.g., "If I am home alone and I feel sad (CS), I will have an eating binge (US)"] and exposing the patient to these exact cues while facilitating attention to both the cues and the non-occurrence of the US (eating). This expectancy violation approach contrasts with the classical habituation approach, in which exposure sessions are targeted on lowering anxiety (or eating desires). Habituation during exposure sessions has been shown to be non-predictive of treatment outcome (Craske et al., 2008; Schyns et al., 2016), though it remains an empirical question whether expectancy violation is important for treatment outcome in cue exposure therapy. Another interesting technique proposed by Craske and colleagues is occasional reinforced extinction: occasionally reinforcing the CS-US contingency might allow reinforced trials to be associated with extinction, leading to attenuated returns of responses. In cue exposure therapy, occasionally eating small amounts of food may reduce overeating by associating eating a small amount of food with no further intake (see Bouton, Woods, \& Pineño, 2004; van den Akker, Havermans, \& Jansen, 2015).

\section{OBJECTIVES}

The main aim of this RCT was to investigate the effects of a cue exposure intervention that is based on the inhibitory learning-based techniques proposed by Craske et al. (2014) and aimed at achieving long-term reductions in overeating and weight in overweight individuals. An active control intervention was included aimed at improving lifestyle. As primary objective, it was studied whether participants who received the cue exposure vs. control intervention would lose more weight, show larger reductions in eating psychopathology and food cue reactivity, and consumed less high-calorie snack foods, both at post-treatment and at follow-up. As a secondary objective, it was studied whether the cue exposure vs. control intervention would show increased response inhibition and self-control, and less attentional bias towards high-calorie foods. Further, it was studied whether the effects on treatment outcome were mediated by expectancy violation, while habituation was not expected to play a role in treatment outcome. Finally, response inhibition and self-control, good sleep, and attentional bias were expected to be treatment outcome moderators. 


\section{MATERIAL AND METHODS}

\section{OVERVIEW OF STUDY DESIGN}

Participants were screened for eligibility and randomized to either an 8-session cue exposure intervention or an active control intervention (Lifestyle+). Measurements were conducted at pre-measurement, during the intervention, at post-measurement, and at three months follow-up (Figure 1). The study was approved by the Ethical Committee of the Faculty of Psychology and Neuroscience of Maastricht University (148_07_10_2013_A3).

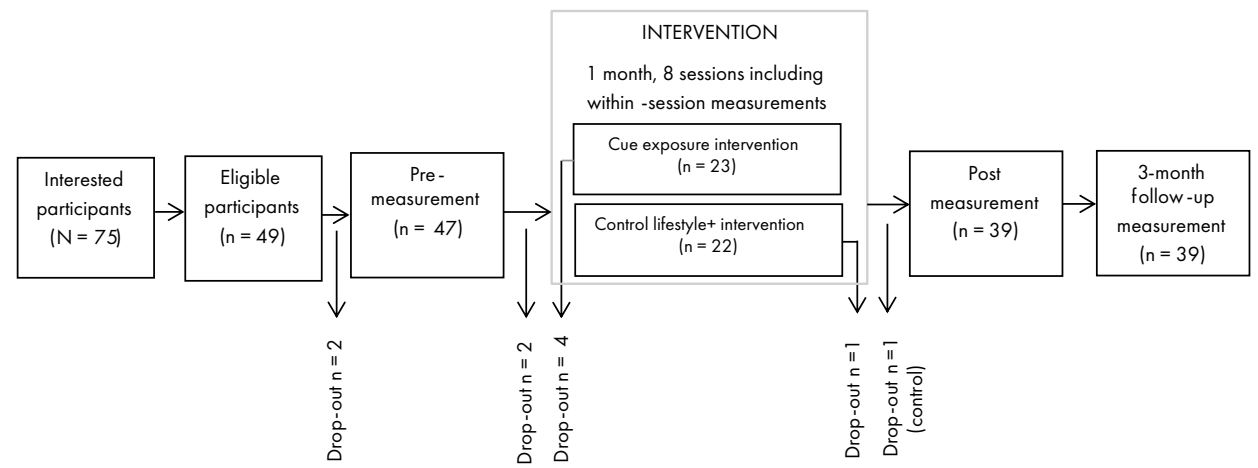

Figure 1. Overview of the study design and sample size.

\section{PARTICIPANTS AND INCLUSION/EXCLUSION CRITERIA}

Participants were overweight and obese individuals. The inclusion criteria included: a female gender, a Body Mass Index (BMI) of at least 27, and an age between 18 and 60 years. A BMI cut-off of 27 was used to avoid including participants who were borderline overweight. Females were included to facilitate comparability with a previous study (Schyns et al., 2016), and to reduce variability in responses. Participants were also required to be highly motivated to lose weight: motivation to lose weight was verbally indicated on a 10 -point scale, and participants had to indicate a minimum motivation of " 8 " to be eligible for participation. In addition, participants had to indicate experiencing a clear difficulty to refrain from eating palatable high-calorie snack foods. The exclusion criteria were: suffering from self-reported smelling problems (indication of anosmia) since smelling is an important part of the cue exposure intervention, pregnancy, currently receiving psychotherapeutic or psychopharmacological treatment, bariatric surgery (pre and postoperative), and insufficient time for the intervention and measurements. As shown in Figure 1, of 75 interested participants that were informed about the study, 49 participants were eligible based on the abovementioned criteria. 


\section{RECRUITMENT AND RETENTION}

Participants were recruited in and around Maastricht through advertisements in local newspapers and gyms, and through flyers. Interested participants were carefully screened for eligibility during a phone interview. Participants received $€ 25$,- for every completed measurement (pre, post, and follow-up). At follow-up, participants received another $€$ 25,- as an additional incentive if they had attended all therapy sessions and measurements. In order to minimize drop-out after the post-measurements, participants were contacted by phone one month before the follow-up measurements to plan the last sessions, and to underline the importance of completing the study. The study was conducted from January 2015 - December 2015.

\section{PROCEDURE}

For each measurement (pre, post, and follow-up), participants attended the university on two separate sessions. It was attempted to minimize the time between both sessions, with a maximum of two weeks.

\section{SESSION 1}

To standardize hunger, participants were asked to consume a small meal (e.g., one sandwich) one hour prior to the start of the session. After arrival, participants received information regarding the set-up of the study and gave written consent. Next, a baseline hunger VAS was completed, followed by baseline food cue reactivity measures (desire to eat VAS, salivation, and prospective portion size). After this, the food cue reactivity task was administered. Participants were exposed to the sight and smell of one of ten snack foods: Lay's salty crisps, Lay's paprika-flavoured crisps, M\&Ms with or without peanuts, Cote d'Or milk chocolate with or without hazelnuts, Cote d'Or dark chocolate, Katja liquorice, Duyvis spiced nuts, and Red Band wine gums. These ten foods were based on the ten most frequently mentioned favourite food items in a female overweight sample from a previous study (Schyns et al., 2016). Participants were instructed to select their favourite food from this list of foods (FF-list). The exposure task lasted exactly three minutes, and the experimenter modelled the exposure. At minute one, participants completed a desire to eat VAS. At minute two, salivation was measured. At minute three, prospective portion size was assessed. After the food cue reactivity task, the Stop-Signal Task was administered, followed by a hunger VAS. Next, only at pre-measurement, participants completed an appetitive conditioning task. At the post and follow-up measurements, participants now completed the bogus taste test. Finally, participants completed another hunger VAS, and the EDE-Q (pre-measurement and follow-up only). 


\section{SESSION 2}

To standardize hunger, participants were asked to consume a small meal two hours prior to this session. Upon arrival, participants completed a hunger VAS. Next, participants completed the attention bias task, wrote down when they last ate, and then completed the Self-Control Scale. After this, the binge eating interview was conducted. Only at premeasurement, this interview was followed by the CS-US interview. The CS-US interview was developed specifically for this study in order to compose the personalized CSUS expectancy items and to identify relevant USs (regular unwanted consumption of unhealthy high-calorie snacks or binge foods) and CSs (e.g., environment, time of the day, feelings, thoughts) for the exposure intervention. The two most favourite snack foods were identified during the interview, of which one would be included (FF-personal exposed) and one would not be included in the exposure exercises (FF-personal non-exposed). After the interview(s), the participant's weight and height was measured.

\section{THERAPY SESSIONS}

At the start of each therapy session (cue exposure and Lifestyle+), a baseline hunger VAS and a desire to eat VAS were administered. In addition, desire to eat VASs was administered every minute during exposure exercises.

\section{MEASUREMENTS}

Primary outcome measures were weight loss, eating psychopathology, food cue reactivity, and snacking behaviour during a bogus taste test. Secondary outcome measures were response inhibition, self-control, and attentional bias. Expectancy violation was investigated as a mediator between condition and treatment outcome. Habituation of food cue reactivity, attentional bias, response inhibition and self-control, and sleep quality were investigated as moderators for treatment success (Craske et al., 2008; Culver, Stoyanova, \& Craske, 2012; Nederkoorn, Jansen, Mulkens, \& Jansen, 2007; Werthmann et al., 2015; Zalta et al., 2013), and interrelations between attentional bias and inhibitory and self-control measures were studied (Hou et al., 2011). An appetitive conditioning task was administered only at pre-measurement for comparison with a normal-weight sample. For an overview of all measurements and the time point of administration, see Table 1. The measurements were conducted by $\mathrm{PhD}$ students and psychology students who were obtaining their master degree. They were blinded to the assigned intervention group. 
Table 1. An overview of all measurements and the time points of administration during the study

\begin{tabular}{|c|c|c|c|c|}
\hline Measures & $\begin{array}{c}\text { Pre- } \\
\text { measurement }\end{array}$ & $\begin{array}{l}\text { During the } \\
\text { intervention }\end{array}$ & $\begin{array}{c}\text { Post- } \\
\text { measurement }\end{array}$ & $\begin{array}{l}\text { Follow-up } \\
\text { measurement }\end{array}$ \\
\hline Weight & $\mathrm{x}$ & & $\mathrm{x}$ & $\mathrm{x}$ \\
\hline Binge eating & $x$ & & $x$ & $\mathrm{x}$ \\
\hline $\begin{array}{l}\text { Eating Disorder Examination Questionnaire } \\
\text { (Fairburn \& Beglin, 1994) }\end{array}$ & $x$ & & & $x$ \\
\hline $\begin{array}{l}\text { Expectancy violation } \\
\text { (CS-US expectancies) }\end{array}$ & $x$ & & $x$ & $x$ \\
\hline Food cue reactivity & $\mathrm{x}$ & & $\mathrm{x}$ & $\mathrm{x}$ \\
\hline $\begin{array}{l}\text { Snacking behaviour } \\
\text { (bogus taste test) }\end{array}$ & & & $x$ & $x$ \\
\hline $\begin{array}{l}\text { Stop-Signal Task } \\
\text { (Houben, Nederkoorn, \& Jansen, 2014) }\end{array}$ & $\mathrm{x}$ & & $\mathrm{x}$ & $x$ \\
\hline $\begin{array}{l}\text { Baumeister Self Control Scale } \\
\text { (Tangney, Baumeister, \& Boone, 2004) }\end{array}$ & $x$ & & $x$ & $x$ \\
\hline $\begin{array}{l}\text { Attentional bias } \\
\text { (Werthmann et al., 2011) }\end{array}$ & $x$ & & $x$ & $x$ \\
\hline Within and between-session habituation & & $\mathrm{x}$ & & \\
\hline $\begin{array}{l}\text { Pittsburgh Sleep Quality Index } \\
\text { (Buysse, Reynolds, Monk, Berman, \& Kupfer, 1989) }\end{array}$ & $\mathrm{x}$ & & $\mathrm{x}$ & $\mathrm{x}$ \\
\hline Appetitive conditioning & $\mathrm{x}$ & & & \\
\hline
\end{tabular}

\section{PRIMARY OUTCOME MEASURES}

Weight loss: Weight loss at post and follow-up measurement was calculated by the change in percentage of weight relative to pre-measurement: a larger negative score reflecting more weight loss.

Eating psychopathology: The Eating Disorder Examination Questionnaire (EDE-Q; Fairburn \& Beglin, 1994) is a widely-used self-report questionnaire that assesses disordered eating behaviours and attitudes over the previous 28 days. It consists of 28 questions which are each scored on a 7-point scale, with higher scores reflecting greater levels of eating psychopathology. Binge eating was further assessed during a semi-structured interview that was created for this study (binge eating interview), using the DSM-V criteria (American Psychiatric Association, 2013).

Food cue reactivity. Food cue reactivity was assessed by measuring desire to eat, salivation and prospective portion size before and after exposure to FF-list. Desire to eat was measured on a VAS ("How strongly do you desire palatable food right now?"), ranging from 0 (no desire at all) to 100 (very strong desire). Salivation was measured using cotton rolls (Hartmann, nr 2, 10×35 mm). Participants placed the cotton roles between the cheek and lower gum on the left and right side for exactly one minute. The dental rolls were kept in a sealed plastic bag, which was weighed before and after saliva collection using a weighing scale accurate to $0.01 \mathrm{~g}$ (Mettler Toledo, PB3002). The prospective portion size task served 
as a behavioural measure of food cue reactivity. In this task, an empty bowl was presented on a computer screen, and participants selected their desired prospective portion size of FF-list. Participants could adjust the amount of FF-list in the bowl: by moving a marker on a horizontal line to the right, the bowl filled, and by moving the marker to the left, the bowl emptied. Participants were instructed to select as much of FF-list as they would like to eat at that moment in time. The amount selected was converted to kcal and served as dependent variable.

Snacking behaviour: To assess snacking behaviour, participants completed a 10-min bogus taste test. The taste test was personalized for each participant, and consisted of three personal favourite snack foods. Two of the test food items were incorporated during cue exposure therapy (FF-list and FF-personal exposed). In addition, a third personal favourite food item was used in the taste test which was not incorporated during cue exposure (FFpersonal non-exposed). The non-exposed food item was included to test whether the effects of cue exposure on food intake would generalize to other, non-exposed, foods. As a cover story, participants were presented with two supposedly slightly different versions of the three types of foods (six bowls of food in total), and were asked to taste these foods and answer questions about the differences between the two versions of each food (e.g., "Which is stronger in flavour?"). In reality, the two 'versions' were identical. Participants were instructed that they can taste as much of the foods as they like, and that they have to remain seated until the experimenter returns.

\section{SECONDARY OUTCOME MEASURES}

Inhibitory control: general response inhibition as well as response inhibition to food was measured using the Stop-Signal Task (SST; e.g., Houben et al., 2014). In the SST, participants performed a go task and a stop task. In the stop task, reactions to the go task had to be inhibited. The go stimuli in the general SST were the letters X and O; the go stimuli in the food SST were pictures of sweet and savoury food. The go stimulus was always presented for $1000 \mathrm{~ms}$, and participants were required to respond as fast as possible to the stimulus by pressing response keys (e.g., press left for $\mathrm{X} /$ sweet and right for $\mathrm{O} /$ savoury). An auditory stop signal was presented on $25 \%$ of the trials, in which case participants were instructed not to respond to the stimulus. The delay between the go stimulus and stop signal was initially set at $250 \mathrm{~ms}$ and then dynamically adapted in order for participants to correctly inhibit $50 \%$ of the stop trials (the delay was increased when participants successfully inhibited their response, and the delay was decreased when participants failed to inhibit their response). For each SST (general and food-specific), participants completed practice blocks (40 trials in total) followed by two test blocks (64 trials each). The dependent measure was the stopsignal reaction time (SSRT), calculated by subtracting the mean stop delay from the mean reactions times. Higher SSRTs indicate worse inhibitory control. Poorer general and food- 
specific response inhibition (as measured by the SST) has been related to impulsivity and higher BMIs (Houben et al., 2014; Logan, Schachar, \& Tannock, 1997; Nederkoorn, Braet, Van Eijs, Tanghe, \& Jansen, 2006; Nederkoorn, Smulders, Havermans, Roefs, \& Jansen, 2006).

Self-control: self-control was measured using the Self-Control Scale (SCS; Tangney et al., 2004). The SCS is comprised of 36 items that are each scored on a 5-point scale. Higher scores indicate higher self-control. Evidence has been reported for the discriminant and convergent validity of the SCS (Duckworth \& Kern, 2011; Maloney, Grawitch, \& Barber, 2012).

Attentional bias to food cues: Attentional bias to food cues was measured using a visual probe task. Eye movements and manual response latencies were recorded as direct and indirect indices of attention allocation. Stimuli were pictures of highly palatable food items, musical instruments, and neutral non-food items. They were presented in pairs and presented next to each other during $2000 \mathrm{~ms}$, followed by a probe in the location of one of the images. Participants were required to respond to the location of the probe by pressing a button. In critical trials, the stimulus pair consisted of a picture of food and a picture of a neutral instrument. Filler trials consisted of a pair of neutral pictures. In total, the task included 120 trials - 80 critical and 40 filler trials. Attention bias scores were derived from the eye movement data, and manual response latencies were based on response latencies to the location of the probes (see Werthmann et al., 2011 for details). Evidence suggests that attention bias for food is causally related to eating behaviour (Werthmann, Jansen, \& Roefs, 2015).

\section{TREATMENT MEDIATORS AND MODERATORS}

Habituation of cue reactivity. In the cue exposure intervention only, self-reported desire to eat $100 \mathrm{~mm}$ VASs were used to assess within (WSH) and between-session habituation (BSH) of self-reported cue reactivity during cue exposure.

Expectancy violation: Eight CS-US expectancies were rated on perceived expectancy of a CS to be followed by the US using $100 \mathrm{~mm}$ VAS, a higher score reflecting a greater perceived expectancy. Items were formulated in "If CS then US" statements. Four CS-US expectancy items were standard for every participant, and four items were personalized based on the CS-US interview (see Table 2). CS-US expectancies have been assessed in previous cue exposure studies (Schyns et al., 2016; Schyns, Roefs, Smulders, \& Jansen, 2018).

Sleep quality: The Pittsburgh Sleep Quality Index (PSQI; Buysse et al., 1989) was used to measure sleep quality. The PSQI is a 19-item self-report measure that assesses several dimensions of sleep quality in the past month. Higher scores indicate poorer sleep quality.

Response inhibition, self-control, and attentional bias will also serve as moderators of treatment outcome. 
Table 2. Overview of the eight CS-US expectancy items.

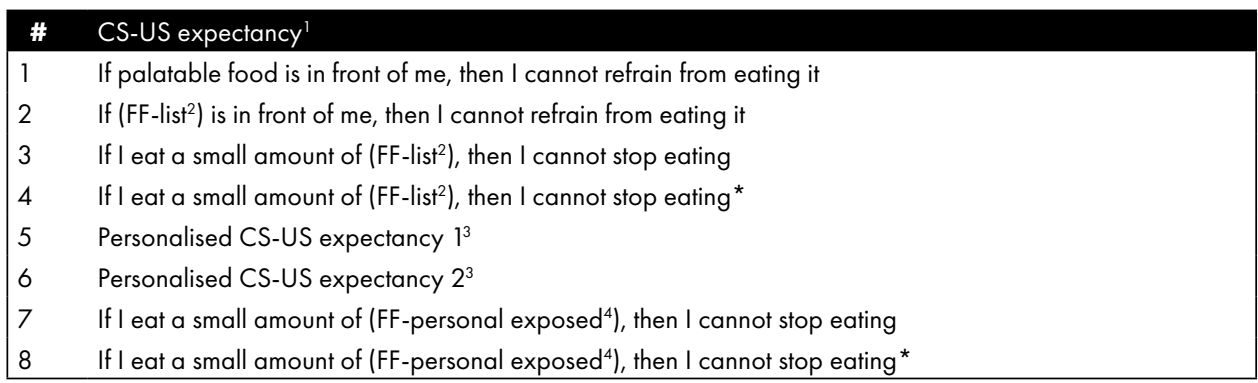

On statements marked with *, participants took a small bite of the specific food before rating their expectancy. 1. CSUS expectancy was scored on a VAS, and ratings ranged from 0 (not at all) to 100 (very strongly). 2. 'FF-list' indicates the favourite food item selected from a list of ten snack foods. 3. 'FF-personal exposed' refers to a favourite personal snack food as assessed during the CS-US interview and utilized as exposed food item. 3. Personalized statements 5 and 6 are based on the CS-US interview (e.g., "If I feel exhausted and have excess to snack foods, then I will have a binge").

\section{TREATMENT ACCEPTABILITY}

Immediately after receiving the rationale of the intervention during the first therapy session, but prior to its start, participants were asked to rate their expectations of the intervention's effectiveness. Similar questions were administered after the intervention at post measurement: "how appropriate will / was this intervention (be) for your complaints"; "how helpful will / was this intervention (be) in diminishing your complaints"; "would you recommend this intervention to a good friend?" All items were rated on a 9-point scale from 1 (not at all) to 9 (very much).

\section{INTERVENTION}

\section{THERAPISTS}

Both interventions were delivered individually and conducted by $\mathrm{PhD}$ students and clinical psychology students who were obtaining their master degree. These students were not involved in the measurements. The therapists adhered to detailed study manuals, and received an extensive training for each intervention. Therapists participated in supervised meetings twice a week in order to discuss treatment progress and potential difficulties of individual participants. Sessions were audiotaped and listened to by the authors to ensure protocol adherence, and used for questions and feedback during the meetings. 


\section{SIMILARITIES OF BOTH INTERVENTIONS}

Both interventions consisted of eight individual therapy sessions that took place during approximately one month (two sessions per week). In addition, all participants received psycho-education on the importance of good sleep quality for decreasing food consumption and BMI (e.g., Baron, Reid, Kern, \& Zee, 2011). Further, for all participants, unhealthy snacking behaviour was strongly discouraged: snacking should be kept to a minimum during the intervention period. Participants in both interventions also received homework, as specified below.

\section{CUE EXPOSURE}

The cue exposure intervention consisted of eight separate face-to-face sessions, and were designed according to the recommendations proposed by Craske et al. (2014). These techniques were translated to cue exposure as described in Table 3. A brief overview of the content of each exposure session is presented in Table 4. Next to the exposures during the therapy sessions, participants were instructed to do daily exposure exercises at home or other contexts. These homework exercises were prepared during the session with the therapist, and each completed exercise was registered. In case the participant would experience irresistible cravings at home and strongly believed she would engage in snacking behaviour ( $\$ 3.2$ ), she was instructed to follow a personal 'emergency card' that was composed together with the therapist in order to avoid unwanted reinforcement of existing CS-US associations. This was done by having the participant consume a different palatable food in an environment not previously associated with intake (e.g., the bathroom).

\section{LIFESTYLE+ CONTROL}

The Lifestyle+ intervention also consisted of eight sessions, including four face-to-face sessions (two at the university and two at the participant's home) that were alternated with four sessions via telephone. The basic components of the Lifestyle+ intervention included extensive healthy lifestyle advice, mindfulness, power-posing exercises (e.g., Cuddy et al., 2015), and psycho-education on body image (e.g., Owen \& Spencer, 2013). Table 4 provides a brief overview of the content of each session. Daily homework exercises consisted of mindfulness and an exercise related to the content of the previous session. 
Table 3. Translation of the techniques as recommended by Craske et al. (2014) to food cue exposure Technique Translation

Expectancy From the CS-US interview, the therapist received a report of the participant's favourite foods violation (USs) and food cues (CSs). Exposures were designed to maximally violate the personal CS-US expectancies: the therapist designed exposure exercises in a way that would maximize the perceived likelihood of the US to occur during exposure. Specific expectancies were assessed by the therapist before the start of exposure, and right after exposure to reflect on the discrepancy between what was expected and what was learned.

Example: Lucy had the expectation to have a binge (US) when feeling exhausted and hungry after a stressful day of work and when high-calorie foods were in the cupboard (CSs). Exposures entailed feelings of exhaustion and hunger by planning the session after a long working day before dinner, and exposing Lucy to her specific binge food while smelling, touching, and licking it. The expectation of losing control was assessed before exposure, and the outcome (i.e., actual loss of control) was evaluated after the exercise.

Deepened Food cues were combined during cue exposure exercises after conducting exposure with at least extinction one of the cues. During the first two exposure sessions, exposure exercises included more isolated CSs, such as the sight and smell of favourite food. From the third session on, additional relevant CSs were included.

Example: Lucy was exposed in session one and two to chocolate of a specific brand, which was always part of her binge ritual. Exposure was conducted in a neutral laboratory: only smelling, touching and licking of the chocolate was part of the first two exposure sessions. From session three on, exposures took place in her living room, where additional CSs were carefully included during the exposure exercise, such as the sight and smell of different food items, the presence or absence of specific family members, and watching a specific TV show.

Multiple Exposure sessions took place in various external (e.g., the university, home environments, fast food contexts restaurants) and internal (e.g., satiety) contexts.

Example: After two exposure sessions in the laboratory, Lucy's sessions were planned at her home context, in her car after grocery shopping, and at the snack bar near her home. Further, exposures were done at varying levels of satiety (see Variability).

Occasional Participants occasionally took one bite of the food during exposure exercises. This occurred once reinforced every exposure session.

extinction Example: The therapist asked Lucy to take a small bite (i.e., a few grams) of the (binge) food at a random time-point during the exposure.

Removal of Safety signals were identified and removed during the exposure sessions.

safety signals Example: Lucy indicated that she would not lose control as long as the therapist was present. Therefore, during exposure sessions, the therapist sometimes left the room. In addition, exposure exercises were done at home without the presence of the therapist. Food for exposure exercises at home was at first provided by the therapist in a plastic container that Lucy needed to return on the next session, while during the course of therapy, Lucy bought her own food and practiced with her own plates, bowls, etc.

Variability Level of satiety, type of food items, time of day, and length of the exposure sessions were measured and varied across sessions.

Example: Lucy's exposures varied in length (between $5 \mathrm{~min}-40 \mathrm{~min}$ ), were planned on various times of day, and included various types of food items (e.g., chocolate bars, hamburgers, crisps). Over the sessions, it also became clear that Lucy's hunger was relatively stable on an intermediate level. To vary satiety levels, meals were planned right before or after the exposure sessions.

Retrieval cues An instructional retrieval cue was used: participants were encouraged to mentally reinstate what was learned so far by writing down the main learning experiences and regularly rehearsing these outside the therapy sessions. This instructional retrieval cue was included from the third session on.

Example: After completing the third session, Lucy wrote down her main learning experiences: she learned that she was able to control food intake, even in her 'weakest' moments when feeling exhausted, stressed and hungry. Further, she learned that food cravings eventually diminish without eating, which was in contrast to her expectation that only eating could relieve her cravings. She discussed her learning experiences with the therapist, and regularly read the assignment during the course of therapy. 
Table 4. Short description of the content of the cue exposure and lifestyle+ control interventions, displayed per session (sessions 1 to 8).

\begin{tabular}{|c|c|c|c|c|c|}
\hline \multicolumn{2}{|c|}{ Cue exposure intervention * } & \multicolumn{4}{|c|}{ Lifestyle+ control intervention } \\
\hline $3-8$ & 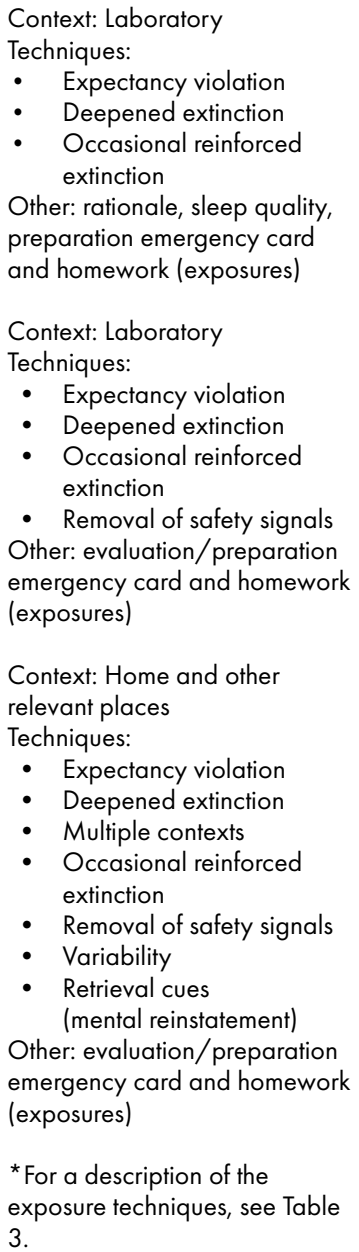 & 3 & $\begin{array}{l}\text { Context: Laboratory } \\
\text { Content: } \\
\text { - Dieting and healthy weight } \\
\text { loss } \\
\text { - Nutrients and energy } \\
\text { balance } \\
\text { - Satiety signals, tips to slow } \\
\text { down eating } \\
\text { - Mindfulness exercise } \\
\text { Other: rationale, sleep quality, } \\
\text { and preparation homework } \\
\text { (energy balance and } \\
\text { mindfulness) } \\
\text { Context: Phone } \\
\text { Content: } \\
\text { - Nutrients and energy } \\
\text { balance } \\
\text { Other: Evaluation and } \\
\text { preparation homework } \\
\text { (energy balance and } \\
\text { mindfulness) } \\
\text { Context: Laboratory } \\
\text { Content: } \\
\text { - Power-posing } \\
\text { - Mindfulness exercise } \\
\text { Other: Evaluation/preparation } \\
\text { homework (power-posing and } \\
\text { mindfulness) } \\
\text { Other: Evaluation/preparation } \\
\text { homework (power-posing and } \\
\text { mindfulness) } \\
\text { Context: Phone } \\
\text { - Powt: } \\
\text { Q Q }\end{array}$ & 7 & $\begin{array}{l}\text { Context: Home } \\
\text { Content: } \\
\text { - Media and thin-ideal } \\
\text { - Body image and well- } \\
\text { being } \\
\text { - Mindfulness exercise } \\
\text { Other: Evaluation/preparation } \\
\text { homework } \\
\text { (thin-ideal and mindfulness) } \\
\text { Context: Phone } \\
\text { Content: } \\
\text { - Thin-ideal } \\
\text { - Q\&A } \\
\text { Other: Evaluation/preparation } \\
\text { homework } \\
\text { (thin-ideal and mindfulness) } \\
\text { Context: Home } \\
\text { Content: } \\
\text { - Fat-talk and Photoshop } \\
\text { - Body image and weight } \\
\text { - loss } \\
\text { - Mindfulness exercise } \\
\text { Other: Evaluation/preparation } \\
\text { homework } \\
\text { (fat-talk and mindfulness } \\
\text { Context: Phone } \\
\text { Content: } \\
\text { - Fat talk } \\
\text { Other: Evaluation homework } \\
\text { (fat-talk and mindfulness) } \\
\text { (a) }\end{array}$ \\
\hline
\end{tabular}

\section{STATISTICAL ANALYSES}

Weight change and expectancy violation at post-measurement and follow-up will be calculated by the change in percentage of weight / CS-US expectancy relative to premeasurement. The mediating role of expectancy violation will be investigated by using the bootstrapping method as described by Preacher and Hayes (Preacher \& Hayes, 2008). Further, independent samples t-tests and repeated-measures ANOVAs will be used to 
examine the effects of treatment group on the other continuous outcome measures. The moderating role of habituation of desire to eat (WSH and $\mathrm{BSH}$ ) on treatment outcome in the exposure intervention will be investigated using correlations. WSH will be operationalised by subtracting the end-level of desire to eat from the peak response of desire to eat during each session and averaging the scores for all sessions. BSH will be calculated by subtracting the peak response from session eight from the peak response of session one (Craske et al., 2008). The other moderators of treatment outcome (change in inhibitory and self-control, attentional bias, and sleep quality) will be investigated using ANOVAs and correlational analyses. Lastly, associations between attentional bias and inhibitory and self-control will be examined using correlations.

\section{DISCUSSION}

The main aim of this study was to examine the effects of a novel cue exposure intervention aimed at maximizing inhibitory learning, relative to an active control intervention. It was expected that participants assigned to the cue exposure vs. control intervention would lose more weight, show larger reductions in eating psychopathology and food cue reactivity, and consume less high-calorie snack foods, both at post-treatment and follow-up. As secondary aim, it was examined whether response inhibition, self-control, and attentional bias towards high-calorie foods showed greater reductions in the cue exposure vs. control intervention. It was further expected that treatment outcome would be mediated by increased CSUS expectancy violation, while habituation was not expected to play a role in treatment outcome. Finally, it was expected that treatment outcome was moderated by good sleep, high response inhibition and self-control, and low attentional bias towards high-calorie food.

The findings of the relatively few cue exposure studies that have been conducted are promising. However, these studies have often not examined weight loss, and some findings indicate returns of cravings and overeating at follow-up - suggesting cue exposure therapy still requires optimization (Boutelle et al., 2014; Jansen et al., 1992, 1989; Martinez-mallen et al., 2007; Schyns et al., 2016; Toro et al., 2003). The present study attempts to address these issues by examining weight loss and by attempting to improve (long-term) outcomes. Specifically, we translated learning theory-based techniques proposed by Craske et al. (2014) for the treatment of anxiety disorders to cue exposure therapy to reduce overeating and obesity, and tested the effectiveness of this inhibitory learning approach. The treatment was personalized for each participant: exposure exercises took place using the participant's CSs (physical contexts, times of day, mood, satiation states, presence or absence of family members) and USs (favourite high-calorie foods). Other notable strengths of the study are 
the inclusion of an active control treatment, a variety of measurements relevant to the study of overeating, an examination of underlying mechanisms, the use of experimenters who were blinded to the participant's condition, and only some drop-out ( $n=6$ after the start of the intervention). One limitation of the study is a limited sample size and power. In addition, it is not clear to what extent findings generalize to male, under-aged and clinical samples. Nevertheless, the current paper provides a novel treatment protocol that can potentially improve current obesity treatments.

\section{CONFLICT OF INTEREST}

The authors report no conflict of interest.

\section{FUNDING}

This work was supported by the Netherlands Organisation for Scientific Research [Vici Grant 453.10.006, awarded to Anita Jansen]. 




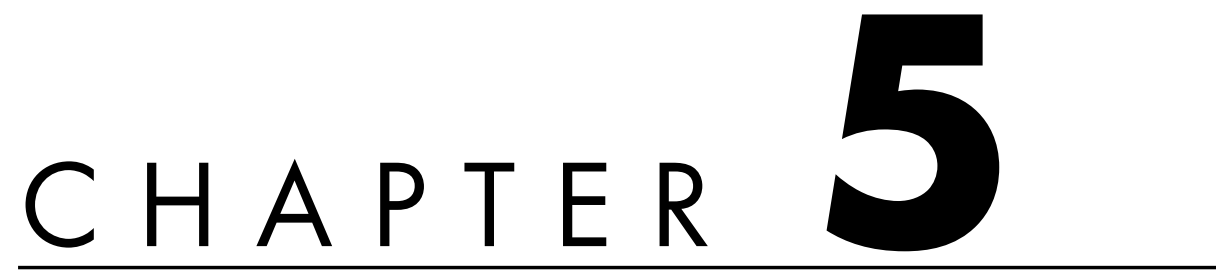

\section{WEIGHT LOSS AND BINGE EATING REDUCTION AFTER EIGHT SESSIONS FOOD CUE EXPOSURE THERAPY AIMED AT INHIBITORY LEARNING IN OBESITY}

Schyns, G., van den Akker, K., Roefs, A., Houben, K., \& Jansen, A. Weight loss and binge eating reduction after eight sessions food cue exposure therapy in obesity. In revision at Journal of Consulting and Clinical Psychology 


\section{ABSTRACT}

OBJECTIVE: The learning theory of overeating predicts that cue exposure therapy is effective in reducing food cue reactivity and overeating. This study tested the effects of an eight session food cue exposure therapy vs. a lifestyle intervention for obesity. Main outcomes were weight loss, food cue reactivity, snacking behavior and eating psychopathology. Expectancy violation, habituation of eating desires and sleep quality were assessed on mediating and moderating effects.

METHOD: After pre-measurement, 45 overweight women were randomly assigned to the cue exposure intervention or the active control condition, both aimed at weight loss. Main outcomes were re-assessed at post-treatment and 3-month follow-up.

RESULTS: Compared to the control condition, participants in the cue exposure condition lost significantly more weight at post-treatment and follow-up, showed a stronger reduction in binge eating frequency, and consumed less kcal of the exposed food item during a bogus taste test, though this effect did not generalize to the non-exposed food item. Expectancy violation mediated the effect of condition on kcal consumption of the exposed food item, while the degree of habituation in the cue exposure condition was not related to better treatment outcome. Sleep quality did not moderate the effect of condition on treatment outcome.

CONCLUSIONS: Eight sessions of food cue exposure reduced body weight, binge eating frequency and snacking behavior, and is therefore a recommendable treatment component to improve obesity treatments.

KEYWORDS: exposure therapy; inhibitory learning; expectancy violation; obesity; weight loss 


\section{INTRODUCTION}

While a large proportion of overweight and obese individuals engage in weight loss attempts (Bish et al., 2005), successful weight loss seems difficult to achieve: About $20 \%$ of overweight individuals are successful at losing at least $10 \%$ of their initial weight and maintaining this weight for at least one year (Wing \& Phelan, 2005). Eating for hedonic purposes instead of physiological needs is a major cause of weight gain (Lowe \& Butryn, 2007). Food-associated cues in the environment (e.g., the sight of Italian ice-cream, or the smell of freshly baked waffles) are theorized to play an important role in hedonic eating, by initiating food cue reactivity (Jansen, Houben, \& Roefs, 2015). This food cue reactivity includes psychological (i.e., craving) and physiological (e.g., salivation) responses, and promotes (over)eating. Indeed, cue-induced food cravings have been shown to be prospectively related to eating and weight gain (Boswell \& Kober, 2016), while reactivity was found to be reduced in successful weight loss maintainers (Jansen, Stegerman, Roefs, Nederkoorn, \& Havermans, 2010). These findings suggest that successfully refraining oneself from consuming highcaloric foods is associated with decreased food cue reactivity, which in turn might make it easier to resist tempting foods and hence promotes weight loss (Jansen, Schyns, Bongers \& van den Akker, 2016).

Models of overeating state that food cue reactivity is at least partly learned (e.g., Jansen, 1998; Jansen, Schyns, Bongers, \& van den Akker, 2016): Food cues (conditioned stimuli; CSs) have become associated with eating (unconditioned stimulus; US) through repeated pairings, and these CSs can elicit cue reactivity. Similarly, learning models predict that extinction of food cue reactivity can be achieved through repeated exposure to CSs without the US (eating), thereby lowering cue-elicited motivation to eat (e.g., Jansen, 1998; van den Akker, Havermans, Bouton, \& Jansen, 2014). A clinical translation of this learning model of overeating is food cue exposure therapy, in which individuals who overeat and/or binge eat are repeatedly exposed to personalized food cues (CSs) without eating (US). Pilot studies in bulimia nervosa patients show substantial reductions in cue-elicited cravings and binge eating after therapy (Jansen, Broekmate, \& Heymans, 1992; Jansen, Van den Hout, De Loof, Zandbergen, \& Griez, 1989; Martinez-mallen et al., 2007; Toro et al., 2003). In overweight samples, cue exposure has been shown to reduce overeating during a bogus taste test of general snack foods (Boutelle et al., 2011, 2014). Also in an overweight sample, it has been found that cue exposure specifically leads to less overeating of food items included in exposure therapy (exposed foods), but not of food items not included in exposure (generalization) (Schyns, Roefs, Mulkens, \& Jansen, 2016). While cue exposure has been found to prevent weight regain after successful weight loss (Mount, Neziroglu, \& Taylor, 1990), it is unclear whether cue exposure therapy is also effective in facilitating weight loss, which is the goal of the present study. 
It is also not clear what the working mechanisms of food cue exposure therapy are. In anxiety, it has been proposed to focus in therapy on strengthening inhibitory learning between a fear-associated stimulus (CS; e.g., a dog) and the non-occurrence of an aversive outcome (US; e.g., being bitten; Craske, Treanor, Conway, Zbozinek, \& Vervliet, 2014) instead of aiming at habituation of the fear response. Although CS-US associations involved in anxiety are content-wise distinct from CS-US associations involved in overeating and obesity, learning principles in the acquisition and extinction of such associations might essentially be the same. In fact, Peters and colleagues (2009) have demonstrated an overlapping neural circuitry involved in extinction in fear and reward memories, suggesting a common mechanism and the possibility of a transdiagnostic treatment approach for anxiety- and reward-related disorders. In line with this transdiagnostic approach, similar to anxiety studies, stronger habituation of food cue reactivity during one cue exposure session was found not to be associated with beneficial treatment outcome (Schyns et al., 2016). That is, larger habituation of self-reported eating desires and salivation during the exposure session did not significantly correlate with overeating.

Based on the inhibitory learning approach, a number of exposure techniques have recently been proposed, which should maximize inhibitory learning and hence, improve the effectiveness of exposure therapy for anxiety disorders (Craske et al., 2014). A food cue exposure therapy developed for eating disorders (Jansen, 1998) and obesity (Jansen et al., 2016) was used in the present study and, based on recent extinction insights, we included the new exposure techniques in the protocol. One recommendation proposed by Craske et al (2014) is to design exposure sessions in a way that maximizes the mismatch between the expectancy of what will happen during exposure and what actually happens (i.e., the expected catastrophic event does not occur). This violation of CS-US expectancies could be achieved in food cue exposure by identifying a patient's specific overeating cues that are linked to strong eating expectancies (e.g., "If I feel exhausted and chocolate is available [CS], then I will lose control and eat the entire chocolate bar [US]"), and exposing that patient to these exact cues while testing whether the US indeed takes place as expected. Exposure therapy for panic disorder aimed at expectancy violation has been shown to be superior to exposure aimed a habituation of anxiety (Salkovskis, Hackmann, Wells, Gelder, \& Clark, 2006) and continuing exposure therapy until the expectancy of the feared outcome was maximally 5\% led to better outcomes than ending at higher expectancies (Deacon et al., 2013). To date, no food cue exposure studies have aimed exposure sessions on expectancy violation, or have compared the expectancy violation approach to the traditional habituation approach. While lower CS-US expectancies after one exposure session have been shown to be related to reduced intake in adults (Schyns et al., 2016), individual CS-US expectancies were not explicitly tackled in that study, but instead used a standard-length exposure session in which eating desires were continuously increased as much as possible. 
In addition to aiming cue exposure sessions at expectancy violation, other recommended techniques by Craske et al (2014) and Jansen (1998; Jansen et al., 2016) seem important to include in cue exposure as well, for example aimed at the prevention of relapse of behaviours after extinction. Given that the original CS-US association remains intact, there are a number of risk factors that can cause this old association to pop-up (Bouton, 1988, 2011). One risk factor is a change of context, called renewal. Solutions to limit renewal are to extent extinction learning to other relevant contexts, or to include mental 'reminders' of extinction, so that the participant actively remembers extinction in a different context. Another important risk of relapse is reinforcement of the original CS-US association, referred to as rapid reacquisition. This type of relapse might be especially important to take into account in cue exposure therapy, as appetitive associations, in contrast to associations in anxiety disorders, are likely to get reinforced again in the future (Bouton, 2011). As a solution to rapid reacquisition, it has been proposed to included occasional US reinforcements during extinction, which can be translated to practice by occasionally instructing participants to take a small bite during cue exposure exercises (Bouton, Woods, \& Pineño, 2004; van den Akker, Havermans, \& Jansen, 2015). Other techniques have been proposed as well, including deepened extinction (i.e., extinction of CSs separately before combining multiple CSs during extinction), removal of safety signals, and variability of various therapy-related factors (Craske et al., 2014).

The current study investigates the effect of cue exposure therapy aimed at inhibitory learning, including all abovementioned strategies. It is hypothesized that food cue exposure will induce more reduction in weight, food cue reactivity, snacking behavior and eating psychopathology than the active control lifestyle treatment. It will also be investigated whether habituation of cue reactivity vs. violation of CS-US expectancies serve as mediators or moderators of treatment outcome. Finally, because good sleep quality has been shown to be related to better treatment outcome in exposure treatment due to enhanced memory consolidation (Pace-Schott, Verga, Bennett, \& Spencer, 2012; Zalta et al., 2013), it is investigated whether sleep quality moderates the effect of treatment on outcome variables.

\section{METHOD}

\section{PARTICIPANTS}

45 female overweight participants $(\mathrm{BMI}>27)$ who were motivated to lose weight, aged 18 to 65 years, were recruited through advertisements. After completing pre-measurements, participants were randomly assigned to the food cue exposure condition $(n=23)$ or the lifestyle control condition $(n=22)$, using sealed envelopes. Six participants dropped out 
during the course of the study intervention (mainly due to the insufficient time for the appointments; cue exposure $n=4$; control $n=2$ ), ending up with 39 participants in total (cue exposure $n=19$; control $n=20$ ). The study was approved by the Ethical Committee of Faculty of Psychology and Neuroscience of Maastricht University.

\section{INTERVENTION}

\section{CUE EXPOSURE INTERVENTION}

The cue exposure intervention consisted of eight individual face-to-face sessions of roughly one hour. The participant's favorite foods and individual expectancies were used for the exposure sessions, including one most favorite food item (i.e., 'personal-exposed food item'). In addition, one food item was selected by the participant from a general list of snack items and also included in exposure therapy (i.e., 'general-exposed food item'; see 2.5.2). Desire to eat was measured at every minute during exposure exercises. Exposures were composed to maximally violate personal CS-US expectancies: the therapist designed exposure exercises in a way that would maximize the perceived likelihood of the US to occur during exposure, while testing whether the US indeed takes place as expected. The first two exposure sessions took place at the university, followed by exposure sessions in various relevant contexts, including a participant's home, snack bars, etc. Occasional reinforcements were included during exposures by taking small bites from the exposure foods. Further, variability of hunger, time of day, and length of exposure sessions were taken into account in scheduling exposure sessions for each participant, and hunger was measured at the start of each session. By combining food cues (such as the smell and sight of food and specific television series, time or mood) during cue exposure exercises after conducting exposure with at least one of the cues, deepened extinction was also attempted. Mental rehearsal was included as a form of mental retrieval cues, and safety signals were excluded as much as possible (such as therapists leaving the room during exposures). Participants were also instructed to do homework exposure exercises between the sessions.

\section{CONTROL INTERVENTION}

The 'Lifestyle+' control intervention consisted of eight sessions, including four face-to-face sessions (two at the university and two at the participant's home) that were alternated with four sessions via telephone. The basic components of the Lifestyle+ treatment included healthy lifestyle advice (extensive dietary advice on how to lose weight), mindfulness, power posing exercises and psycho-education on body image. Daily homework exercises consisted of mindfulness and exercises related to the content of the previous session. During telephone sessions, the homework exercises were extensively evaluated. 


\section{PROCEDURE}

Every participant engaged in a pre-measurement, a post-measurement directly after the intervention, and a follow-up measurement three months after completing the intervention. Measurements were conducted by experimenters blinded to a participant's assigned condition. After completing the pre-measurement, the participant was randomized into the cue exposure vs. control condition. Both interventions consisted of eight individual therapy sessions that took place during approximately one month (two sessions per week). In addition, participants in both conditions received psycho-education on the importance of good sleep quality for weight loss, and unhealthy snacking behavior was strongly discouraged to achieve weight loss. BMI, the Pittsburgh Sleep Quality Index (PSQI; Buysse, Reynolds, Monk, Berman, \& Kupfer, 1989), CS-US expectancy, food cue reactivity and a semi-structured interview to assess binge eating were included in all three measurements. The CS-US interview was done at pre-measurement to compose individual CS-US expectancy items, and to identify both the personally relevant favorite snack foods associated with overeating and the cues that predict the overeating (e.g., seeing and smelling the foods, environments, time of the day, feelings, thoughts) for the exposure intervention. In addition, this interview was used to identify the two personal most favorite snack foods, of which one was included in exposure therapy (personal-exposed food item) and the other one would not be included (personal-non-exposed food item). Snacking behavior was measured at post-measurement and follow-up. The Eating Disorder ExaminationQuestionnaire (EDE-Q; Fairburn \& Beglin, 1994) was administered at pre- and follow-up measurements. The participant rated treatment expectations after the introduction in the first session, while the evaluation of the treatment was assessed at the post-measurement.

\section{GENERAL ASSESSMENTS}

\section{INTERVENTION ACCEPTABILITY}

Prior and after the intervention, the participant rated expectations/ evaluations of the intervention on appropriateness, helpfulness and recommendability to a friend on a 9-point scale from 1 (not at all) to 9 (very much).

\section{BMI}

Height and weight were measured to calculate BMI $\left(\mathrm{kg} / \mathrm{m}^{2}\right)$.

\section{CUE EXPOSURE CHARACTERISTICS}

The number of different foods included in exposure exercises and the number of exposures to the personal-exposed food item and general-exposed food item were recorded, including 
exposures during homework. Furthermore, the level of hunger of each exposure session was measured on a visual analogue scale (VAS; 'How hungry are you right now?'), ranging from 0 (not hungry at all) to 100 (very hungry), and the time of day and length of each exposure session was recorded. The number of homework exercises that were performed between sessions was also recorded.

\section{PRIMARY OUTCOME MEASURES}

\section{WEIGHT LOSS}

Weight loss at post and follow-up measurement was calculated by the change in percentage of weight relative to pre-measurement: a larger negative score reflecting more weight loss.

\section{FOOD CUE REACTIVITY}

To measure food cue reactivity, the most palatable snack item was chosen by the participant from a list of ten snack foods (also included in exposure therapy; 'general-exposed food item'). The participant was exposed to this food item for three minutes. Desire to eat, salivation and prospective portion size (PPS) were measured before and after exposure. Desire to eat was measured on a VAS ('How strongly do you desire palatable food right now?'), ranging from 0 (no desire at all) to 100 (very strong desire). Salivation was measured using dental rolls (Hartmann, $\mathrm{nr} 2,10 \times 35 \mathrm{~mm}$ ), placed between the cheek and lower gum on the left and right side, for exactly one minute. The dental rolls were kept in a sealed plastic bag and weighed before and after saliva collection using a weighing scale accurate to $0.01 \mathrm{~g}$ (Mettler Toledo, PB3002). During the computerized PPS task, the participant indicated how much she would currently like to eat by selecting an amount of food on a computer screen (van den Akker, Bongers, Hanssen, \& Jansen, 2017).

\section{SNACKING BEHAVIOR}

A 10-min bogus taste test was completed, which was personalized for each participant, consisting of three snack foods: the personal-exposed food item, the personal-non-exposed food item and the general-exposed food item. The personal-non-exposed food item was included to test generalization of the exposure effect to favorite non-exposed foods. Foods were weighed before and after the taste test, and the amount of kcal eaten was calculated.

\section{EATING PSYCHOPATHOLOGY}

The 28-item EDE-Q (Fairburn \& Beglin, 1994) was administered to assess eating psychopathology. In addition to the EDE-Q global score, which provides an indication of the severity of eating psychopathology, four subscales on eating, shape and weight concerns, and dietary restraint are assessed, a higher score indicating more severe psychopathology. 
In addition, binge eating during the last week was assessed during a semi-structured interview because self-reports of binge eating appear to be rather unreliable (Fairburn \& Beglin, 1994).

\section{TREATMENT MEDIATORS AND MODERATORS}

\section{HABITUATION OF CUE REACTIVITY}

Self-reported desire to eat was repeatedly measured to assess within (WSH) and betweensession habituation (BSH; in the cue exposure intervention only).

\section{CS-US EXPECTANCIES}

During a semi-structured interview (CS-US interview), individual overeating situations were identified to formulate four personalized CS-US expectancy items in 'If CS then US' statements. CS-US expectancies were rated on expectancy of a CS to be followed by the US using a 100mm VAS, a higher score reflecting a greater expectancy. In addition to four personalized items, four standard CS-US expectancies were assessed (see Table 5 for overview of the items). CS-US expectancy violation at post-measurement and followup were calculated by percentage change relative to pre-measurement: a larger negative percentage reflecting more expectancy violation.

\section{SLEEP QUALITY}

The PSQI (Buysse et al., 1989) was used to measure sleep quality, with higher scores indicating poorer sleep quality during the last month.

\section{STATISTICAL ANALYSES}

Analyses on the comparison of conditions on continuous variables included independent samples t-tests, and mixed model analyses of variance (ANOVAs) for between-subjects and within-subjects variables. If the Levene's test for homogeneity of variances was found to be violated, a t-test not assuming homogeneous variances was reported. Paired-samples t-tests were used to compare the frequency of exposures to the personal-exposed food item and general-exposed food item, and the degree habituation within the cue exposure condition. Regarding habituation, WSH of eating desires in the cue exposure condition was operationalised by subtracting the individual end-level of eating desires from the individual peak desire during each session and averaging the scores for all sessions. BSH was calculated by subtracting the individual peak desire from session eight from the individual peak desire of session one (Craske et al., 2008). Mediational effects of expectancy violation (condition $\rightarrow$ expectancy violation $[\mathrm{M}] \rightarrow$ treatment outcome [weight loss, kcal of personal-exposed 
food item, reduction binge eating]) were tested by applying the bootstrapping method with a $95 \%$ confidence interval of the indirect effect using 5000 samples as described by Preacher and Hayes (2008). Indirect mediation effects are considered significant when the $95 \%$ confidence interval does not contain zero. Associations between habituation of eating desires and treatment outcome were examined using Pearson correlations. Moderation effects of expectancy violation and sleep quality were investigated by calculating condition $\mathrm{x}$ expectancy violation/ sleep quality interactions using one-way ANOVA's.

\section{RESULTS}

\section{DESCRIPTIVES}

The mean BMI at pre-measurement was not significantly different between the cue exposure condition $(M=33.05, S D=3.48)$ and control condition $(M=34.51, S D=5.57), t(37)=0.97$, $p=.337, d=0.32$. This also held for age, with a mean age of 44.26 years $(S D=10.42)$ in the cue exposure and 40.25 years $(S D=13.12)$ in the control condition, $t(37)=1.05, p=.299$, $d=0.35$.

\section{INTERVENTION ACCEPTABILITY AND EVALUATION}

As shown in Table 1, before starting the intervention, expectations of participants about the cue exposure and control intervention were not significantly different. However, after finishing the intervention, the cue exposure intervention was rated as more helpful and recommendable to friends than the control intervention.

Table 1. Mean and standard deviations (SD) of expectations and evaluations of intervention acceptability, separated by condition (cue exposure, control)

\begin{tabular}{|c|c|c|c|c|c|c|}
\hline & \multicolumn{3}{|c|}{ Before intervention } & \multicolumn{3}{|c|}{ After intervention } \\
\hline & $\begin{array}{c}\text { Cue } \\
\text { exposure } \\
M(S D)\end{array}$ & $\begin{array}{l}\text { Control } \\
M(S D)\end{array}$ & $\begin{array}{c}\text { Comparison of } \\
\text { conditions }\end{array}$ & $\begin{array}{c}\text { Cue } \\
\text { exposure } \\
M(S D)\end{array}$ & $\begin{array}{l}\text { Control } \\
M(S D)\end{array}$ & $\begin{array}{l}\text { Comparison of } \\
\text { conditions }\end{array}$ \\
\hline $\begin{array}{l}\text { Appropriate for } \\
\text { complaints }\end{array}$ & $7.26(1.63)$ & $7.60(1.60)$ & $\begin{array}{c}t(37)=0.65 \\
p=.519, d=0.21\end{array}$ & $7.39(1.54)$ & $6.21(2.64)$ & $\begin{array}{c}t(29.27)=1.67 \\
p=.105, d=0.55\end{array}$ \\
\hline $\begin{array}{l}\text { Helpful for } \\
\text { complaints }\end{array}$ & $6.89(1.70)$ & $7.25(1.52)$ & $\begin{array}{c}t(37)=0.69 \\
p=.949, d=0.22\end{array}$ & 7.83 (1.69) & $5.58(2.85)$ & $\begin{array}{c}t(29.50)=2.94 \\
p=.006, d=0.96\end{array}$ \\
\hline $\begin{array}{l}\text { Recommendable } \\
\text { to friend }\end{array}$ & $7.00(1.89)$ & $7.65(1.66)$ & $\begin{array}{c}t(37)=1.14 \\
p=.260, d=0.37\end{array}$ & $8.22(1.44)$ & $6.32(2.94)$ & $\begin{array}{c}t(26.42)=2.52 \\
p=.018, d=0.82\end{array}$ \\
\hline
\end{tabular}




\section{CUE EXPOSURE CHARACTERISTICS}

An average of $7.33(S D=2.17)$ different foods were included in exposure exercises during the course of therapy. The number of exposures was not significantly different between the personal-exposed food item $(M=7.19, S D=6.70)$ and the general-exposed food item $(M=8.05, S D=9.18), t(20)=0.84, p=.413, d=0.18$. Average hunger levels at the start of exposure sessions were $28.15(\mathrm{SD}=18.87$ ), ranging between 0 and $98 \mathrm{~mm}$. Exposure sessions were scheduled between 8 a.m. and 7 p.m. The mean lengths of exposure sessions was 8.91 minutes $(S D=1.35)$ and ranged between five and 22 minutes across sessions. Participants on average did a total number of $28.11(S D=10.16)$ homework exposure exercises during the course of therapy.

\section{PRIMARY OUTCOMES}

\section{WEIGHT LOSS}

As can be seen in Figure 1, participants in the cue exposure intervention $(M=-2.05, S D$ $=2.26$ ) lost a significantly larger percentage of weight compared to the control condition $(M=-0.43, S D=1.74)$ at post-measurement, $t(36)=2.48, p=.018, d=0.83$. Larger weight loss was also found at follow-up measurement for the cue exposure $(M=-2.28, S D=3.97)$ versus control condition $(M=0.36, S D=3.23), t(37)=2.28, p=.029, d=0.75$.

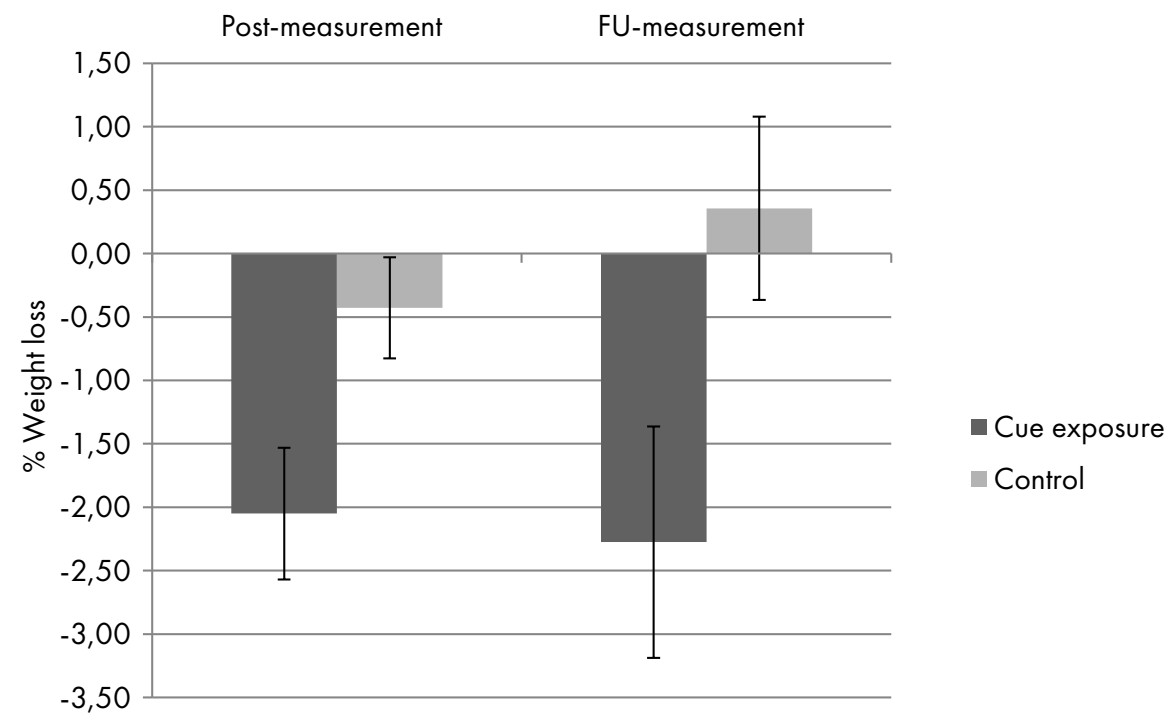

Figure 1. Percentage of weight loss per condition (cue exposure, control) per time point (post and followup measurement). Error bars represent standard errors of means. 


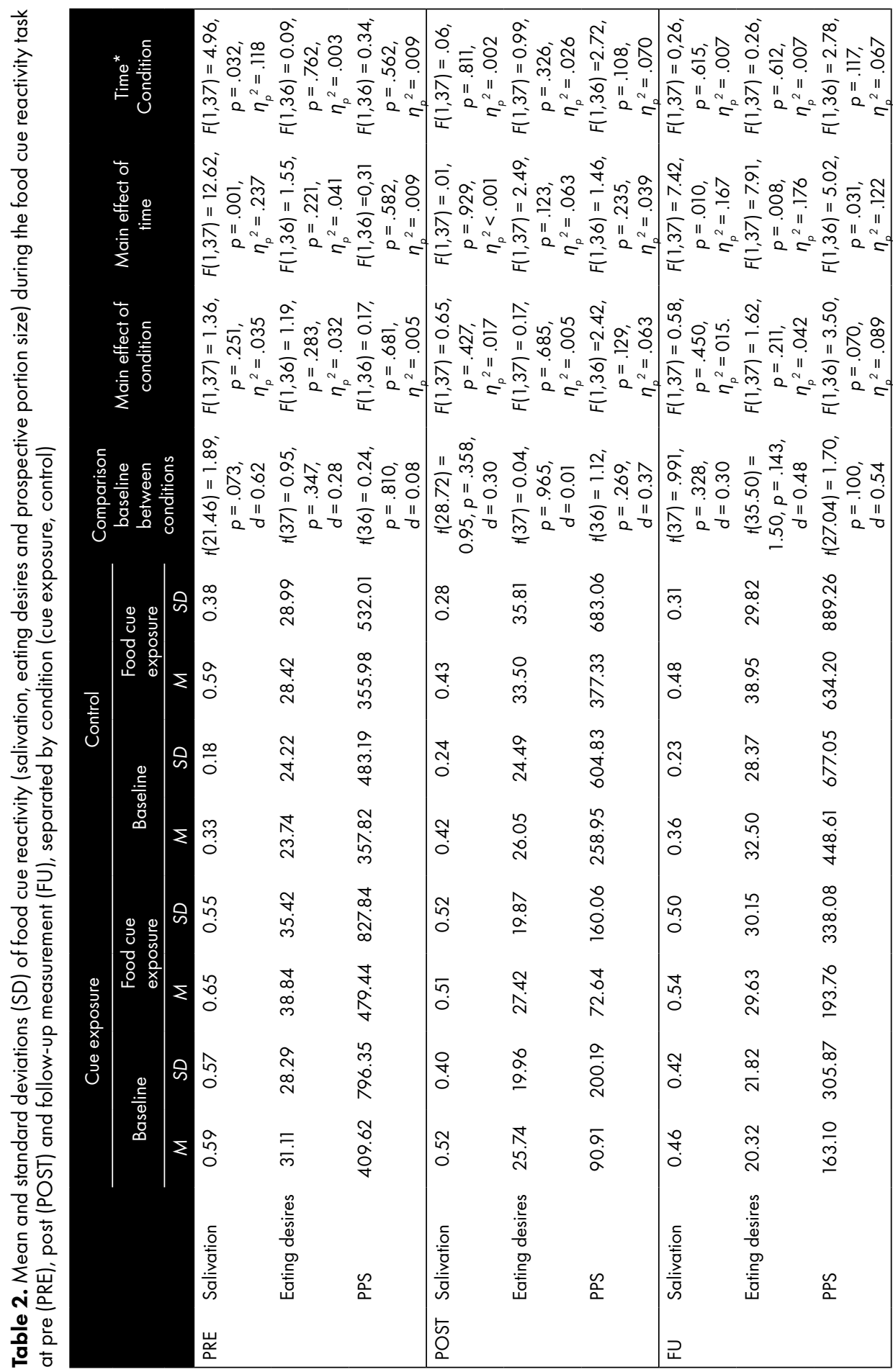




\section{FOOD CUE REACTIVITY}

As can be seen in Table 2, baseline comparisons between conditions on desire to eat, salivation and PPS indicate that both conditions were comparable at pre-measurement, post-measurement and follow-up. As shown in Table 1, cue-induced eating desires and PPS were not successfully increased at pre-measurement during the food cue reactivity task, neither were any significant main effects of condition nor condition $\mathrm{x}$ time (baseline, during the food cue exposure task) interaction effects found. A condition $\mathrm{x}$ time interaction was found for salivation at pre-measurement; further inspection indicated that salivation was significantly increased in the control condition, $t(19)=3.97, p=.001, d=0.89$, but not in the cue exposure condition, $t(18)=0.97, p=.345, d=0.22$. At post-measurement, cue reactivity was not induced in any of the measures, nor were any main effects of condition or time $\mathrm{x}$ condition interaction effects found. In contrast, cue reactivity was successfully induced in all measures at follow-up, as indicated by significant main effects of time on salivation, eating desires and pps. No time $\mathrm{x}$ condition interaction effects were found at follow-up, and neither were main effects of condition found. Although not significant, the average PPS over time points was marginally lower in the cue exposure vs. control condition at follow-up.

\section{SNACKING BEHAVIOR}

As displayed in Table 3, the cue exposure condition consumed significantly less kcal of the personal-exposed food item compared to the control condition at post-measurement, and marginally less at follow-up. Kcal consumption of the personal-non-exposed food item and the general-exposed food item was not significantly different between conditions at posttreatment or at follow-up.

Table 3. Mean and standard deviations (SD) of kcal intake during the bogus taste test at post and followup measurement, separated by condition (cue exposure, control)

\begin{tabular}{|c|c|c|c|c|c|}
\hline \multirow[t]{2}{*}{ Kcal intake } & \multicolumn{2}{|c|}{ Cue exposure } & \multicolumn{2}{|c|}{ Control } & \multirow[t]{2}{*}{ Comparison of conditions } \\
\hline & $M$ & $S D$ & $M$ & $S D$ & \\
\hline \multicolumn{6}{|l|}{ Post-measurement } \\
\hline Personal-exposed food item ${ }^{1}$ & 64.37 & 38.45 & 119.25 & 98.34 & $t(23.38)=2.27, p=.033, d=0.74$ \\
\hline Personal-non-exposed food item ${ }^{2}$ & 93.83 & 92.15 & 89.33 & 47.28 & $t(36)=0.19, p=.851, d=0.06$ \\
\hline General-exposed food item ${ }^{3}$ & 150.38 & 263.42 & 129.74 & 129.25 & $t(34)=0.30, p=.767, d=0.10$ \\
\hline \multicolumn{6}{|l|}{ Follow-up* } \\
\hline Personal-exposed food item ${ }^{1}$ & 70.23 & 53.37 & 110.92 & 89.85 & $t(37)=1.71, p=.096, d=0.55$ \\
\hline Personal-non-exposed food item ${ }^{2}$ & 80.48 & 60.36 & 86.10 & 55.02 & $t(37)=0.30, p=.764, d=0.10$ \\
\hline General-exposed food item ${ }^{3}$ & 94.68 & 84.63 & 120.77 & 123.23 & $t(37)=0.77, p=.448, d=0.25$ \\
\hline
\end{tabular}

1. Personal-exposed food item represents the individually selected item that was included in the exposure intervention. 2. Personal-non-exposed food item represents the individually selected item that was not included in the exposure intervention. 3. General-exposed food item represents the food item selected from a list of ten food items, also included in the exposure intervention. 


\section{EATING PSYCHOPATHOLOGY}

As shown in Table 4, the global EDE-Q score, as well as all concern subscales decreased significantly from pre-measurement to follow-up, as reflected by significant main effects of time. However, no main effects of condition, or time (pre-measurement, follow-up) $\mathrm{x}$ condition interactions were found for any of these subscales or global score. No changes were found for the dietary restraint subscale. $41 \%$ of participants (cue exposure $53 \%$, control 25\%) reported binge eating at pre-measurement. Binge eating frequency at premeasurement was marginally higher in the cue exposure than the control condition, $t(32.21)$ $=1.81, p=.080, d=0.59$. See Figure 2. Interestingly, the number of binges was reduced to zero in all participants in the exposure condition at post-measurement, while this was not the case in the control condition, $t(19.00)=1.83, p=.083, d=0.57$. At follow-up, both conditions reported binge-eating, $t(36)=0.83, p=.412, d=0.17$. Over these three time points, the main effect of condition was not significant, $F(1,35)=2.41, p=.130, \eta_{p}^{2}=.064$, while a significant main effect of time, $F(2,70) 13.59, p<.001, \eta_{p}{ }^{2}=.280$, and a significant time $\mathrm{x}$ condition interaction was found, $F(2,70)=4.69, p=.027, \eta_{p}^{2}=.118$. Follow-up analyses revealed that the reduction of binge eating frequency from pre-measurement to post-measurement was marginally stronger in the cue exposure vs. control condition, $t(31.11)=2.01, p=.053, d=0.65$, and significantly stronger from pre-measurement to follow-up, $t(34.08)=2.26, p=.031, d=0.74$.

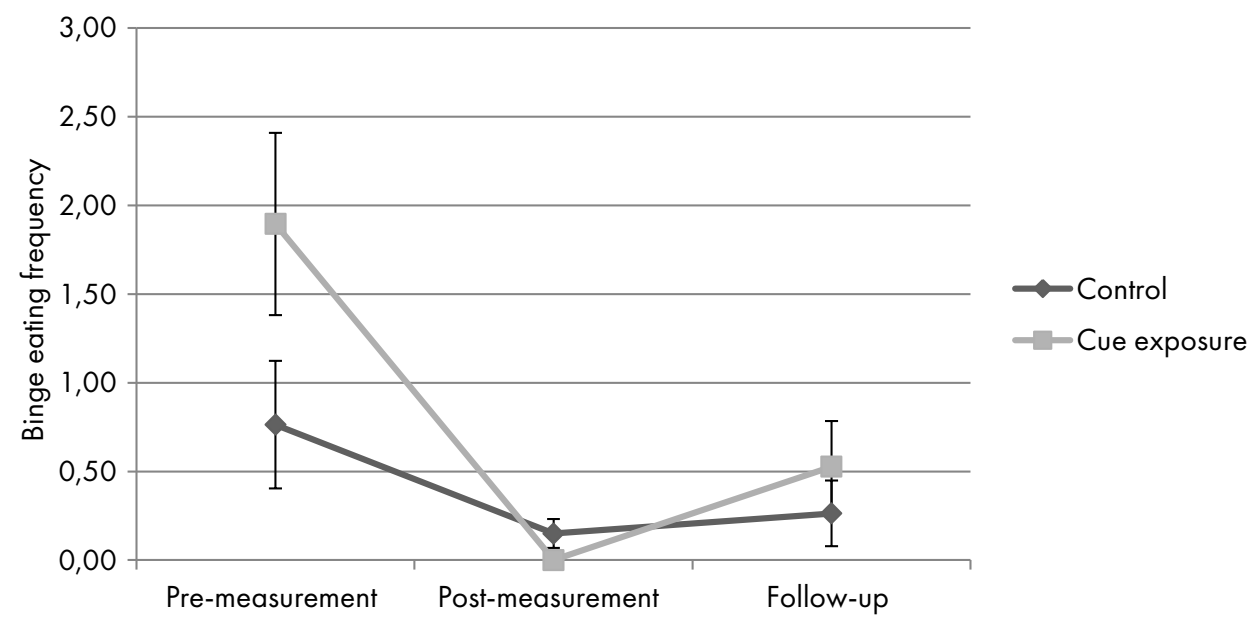

Figure 2. Binge eating frequency during the last 7 days per condition (cue exposure, control) per time point (pre, post and follow-up measurement). Error bars represent standard errors of means. 


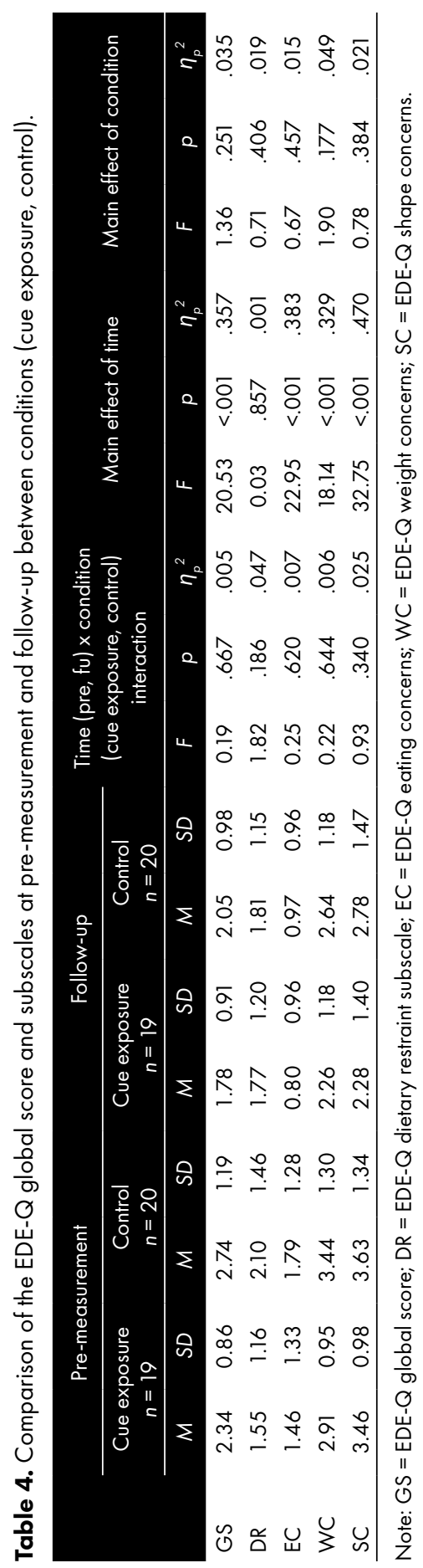




\section{TREATMENT MEDIATORS AND MODERATORS}

To further investigate the findings of significant differential weight change (postmeasurement and follow-up), kcal consumption of the personal-exposed food item (postmeasurement) and binge eating frequency reduction (follow-up) between conditions, the mediating and moderating roles of habituation of desire to eat (in the exposure condition only), expectancy violation and sleep quality were examined.

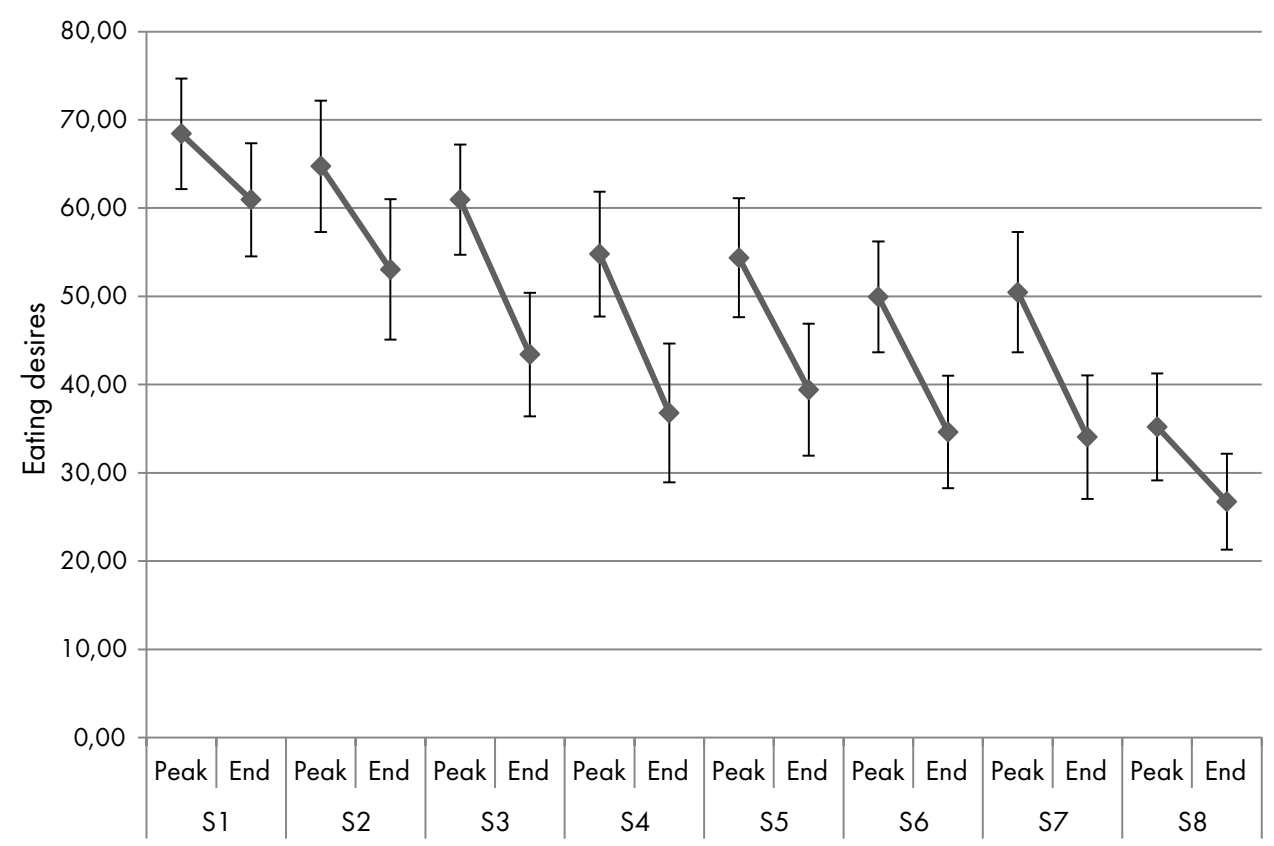

Figure 3. Mean peak and end-level desire to eat scores per session in the cue exposure condition. Error bars represent standard errors of means.

\section{HABITUATION OF CUE REACTIVITY}

Habituation of desire to eat levels during the cue exposure sessions is presented in Figure 3, as reflected by the mean individual peak and end-levels per session. Averaged over sessions, participants in the cue exposure condition experienced a significant within-session decrease (WSH) from peak desire to eat $(M=54.86, S D=23.13)$ to end level $(M=41.13, S D=25.15)$, $t(18)=5.23, p<.001, d=1.23$. In addition, the average peak desire to eat on session one $(M=68.42, S D=27.30)$ was significantly higher than peak desire to eat on session eight $(M=35.21, S D=26.34)$, indicating that on average, participants experienced BSH, $t(18)=$ $4.77, p<.001, d=1.12$. When investigating correlations between habituation and treatment outcome within the exposure condition, BSH did not correlate with percentage weight loss 
at post-treatment, $r(19)=.01, p=.968$, or at follow-up, $r(19)=-.05, p=.846$. BSH also did not correlate with kcal intake of the personal-exposed food item at post-measurement, $r(19)=-.24, p=.326$, or the reduction of binge eating at follow-up, $r(19)=0.14, p=.576$. WSH did significantly positively correlate with weight change at post-measurement, $r(18)$ $=.52, p=.023$, indicating that more WSH was related to more weight gain. However, WSH was neither correlated to weight change at follow-up, $r(18)=.28, p=.255$, nor related to $\mathrm{kcal}$ intake of the exposure food item at post-measurement, $r(18)=-.30, p=.212$, nor the reduction of binge eating at follow-up, $r(19)=0.05, p=.853$.

\section{CS-US EXPECTANCY VIOLATION}

Mean CS-US expectancies are shown in Table 5. Average CS-US expectancy of all eight items at pre-measurement was not significantly different between conditions, $t(35)=$ $.91, p=.371, \mathrm{~d}=0.26$. As can be seen in Figure 4 , expectancy violation of all items at post-measurement was significantly larger in the cue exposure $(M=-68.83, S D=22.80)$ compared to the control condition $(M=-22.81, S D=44.37), t(26.21)=4.06, p<.001, \mathrm{~d}$ $=1.32$. At follow-up, this effect was still observed: cue exposure $(M=-56.72, S D=27.12)$ had significantly larger expectancy violation than the control condition $(M=-36.29, S D=$ 33.91), $t(35)=2.05, p=.048, d=0.67$.

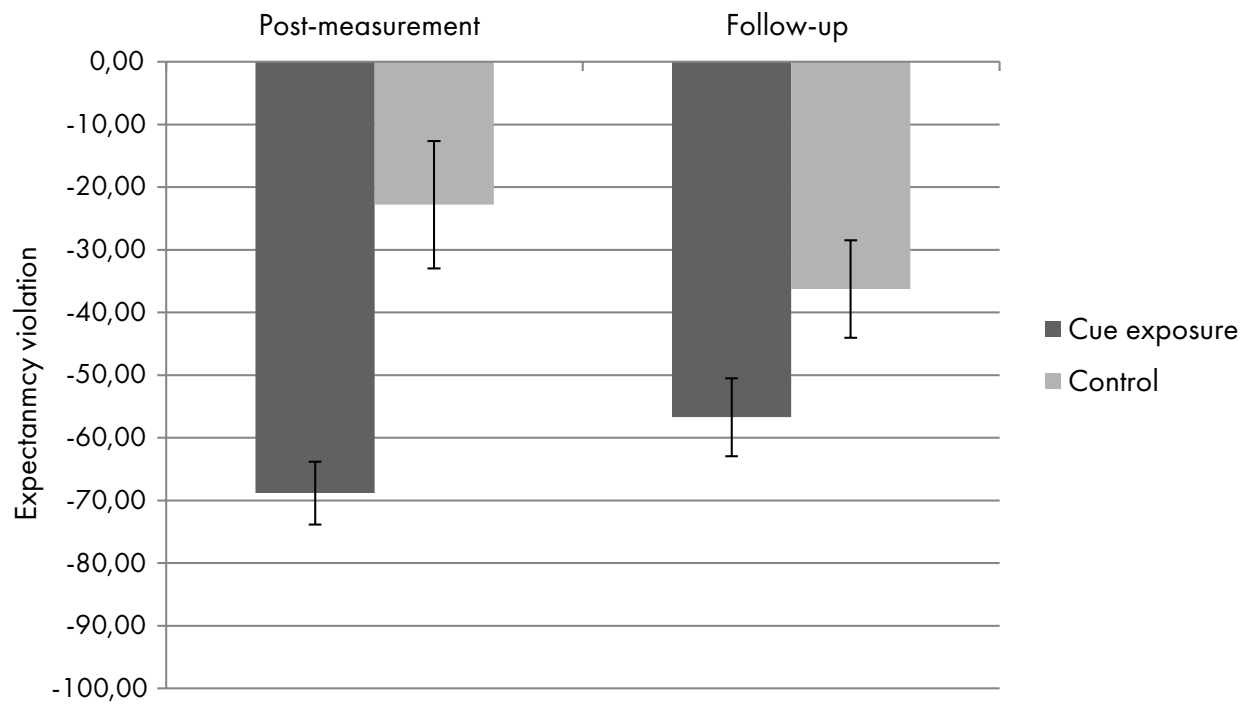

Figure 4. Percentage of expectancy violation relative to pre-measurement per condition (cue exposure, control) per time point (post and follow-up measurement). Error bars represent standard errors of means. 


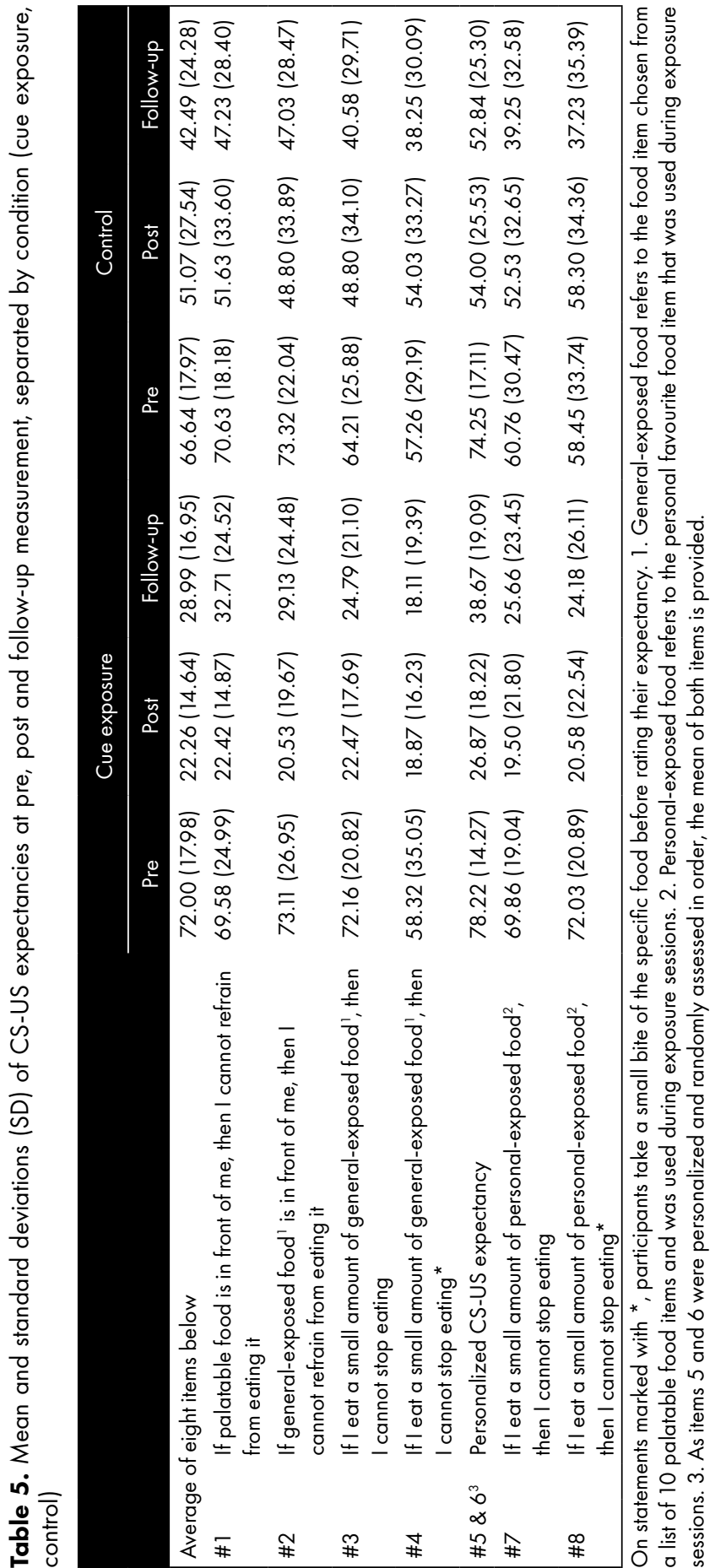


Results of the mediation analyses showed that the indirect effect between condition and weight loss via expectancy violation was not significant at post-measurement, $\beta<0.01$, $95 \%$ CI $[-0.51,0.47]$, nor at follow-up, $\beta=-0.09,95 \%$ CI [-.054, 0.17]. Further, the indirect effect between condition and the reduction of binge eating at follow-up via expectancy violation was also not significant, $b=0.09,95 \% C I[-0.20,0.74]$. Expectancy violation at post-measurement did mediate the relationship between condition and kcal intake at postmeasurement, $\beta=-39.73,95 \% C I[-86.87,-6.03]$. In this model, condition was significantly associated with $\mathrm{kcal}$ (c-path, consistent with 3.4.1; $\beta=-52.11, t(35)=2.11, p=.042$ ), and as also mentioned above, the relationship between condition and expectancy violation was also significant (a-path; $\beta=-45.99, t(35)=3.94, p<.001$ ). The relationship between expectancy violation and kcal (b-path) of this model was statistically significant with a positive coefficient, $\beta=0.84, t(35)=2.52, p=.016$, indicating that less expectancy violation (as a more negative score is stronger violation) was related to more kcal intake. The direct effect between condition and kcal became non-significant when including expectancy violation as a mediator (c'-path), $\beta=-13.35, t(35)=0.48, p=.633$, suggesting full mediation.

Regarding moderation effects of expectancy violation, no main effects of expectancy violation were found for weight loss at post-measurement, $F(1,34)=0.02, p=.658, \eta_{p}^{2}<$ .01 , or follow-up, $F(1,35)=2.50, p=.123, \eta_{p}^{2}=.07$, neither were condition x expectancy violation interactions found for either weight loss at post-treatment, $F(1,33)=1.29, p=$ $.265, \eta_{p}^{2}=.04$, or follow-up, $F(1,34)=0.12, p=.734, \eta_{p}^{2}<.01$. A significant main effect of expectancy violation at post-measurement was found for kcal intake of the personalexposed food item, $F(1,34)=6.37, p=.016, \eta_{p}{ }^{2}=.16$, though this effect was not qualified by a condition $\mathrm{x}$ expectancy violation interaction, $F(1,33)=0.06, p=.813, \eta_{p}{ }^{2}<.01$. Lastly, there was no significant main effect of expectancy violation or condition $\mathrm{x}$ expectancy violation interaction on binge eating reduction at follow-up, $F(1,33)<.01, p=.924, \eta_{p}^{2}<$ .01 , and $F(1,32)=0.06, p=.807, \eta_{p}^{2}<.01$, respectively.

\section{SLEEP QUALITY}

Sleep quality improved, which is demonstrated by the significant decrease in mean PSQI score from pre-measurement (cue exposure: $M=6.44, S D=3.46$; control: $M=5.55, S D$ $=3.03$ ) to post-measurement (cue exposure: $M=5.53, S D=3.08$; control: $M=3.95, S D$ $=2.11$ ) and follow-up (cue exposure: $M=4.80, S D=3.21$; control: $M=5.79, S D=3.91$ ) in both conditions, as reflected by a significant main effect of time, $F(2,33)=5.93, p=$ $.006, \eta_{p}^{2}=.264$, and the absence of a main effect of condition, $F(1,34)=1.22, p=.277$,

$\eta_{p}{ }^{2}=.034$ or a condition $\mathrm{x}$ time interaction, $F(2,33)=0.49, p=.620, \eta_{p}{ }^{2}=.029$. Sleep quality at post-treatment, reflecting sleep during the month of the intervention, was no significant moderator of kcal intake (personal-exposed food item) and weight loss at postmeasurement, since condition $\mathrm{x}$ sleep interactions were not significant (weight loss: $F(1,34)$ 
$=1.93, p=.174$; kcal personal-exposed food item: $\left.F(1,34)=1.14, p=.294, \eta_{p}^{2}=.054\right)$. Sleep quality also had no main effect on weight loss, $F(1,35)<0.01, p=.958, \eta_{p}^{2}<.001$, or kcal intake of the personal-exposed food item, $F(1,35)=0.16, p=.691, \eta_{p}^{2}=.132$.

\section{DISCUSSION}

This study tested the effects of eight sessions of cue exposure therapy aimed at inhibitory learning (Craske et al., 2014) on several important outcomes for overweight and obese females, and was compared to an active control intervention. The mediating and moderating role of expectancy violation vs. habituation of eating desires on treatment outcome were also investigated. It was found that participants in the cue exposure condition lost significantly more weight at post-measurement and follow-up than participants in the control condition. Further, participants in the cue exposure condition had a marginally larger reduction of binge eating at post-measurement and significantly larger reduction of binge eating at follow-up. The cue exposure vs. control condition also consumed significantly less kcal of the personalexposed food item at post-treatment and marginally less kcal at follow-up, though no differences were found for the personal-non-exposed food item or the general-exposed food item. Expectancy violation was significantly larger in the cue exposure condition compared to the control condition, and was a significant (full) mediator for kcal consumption of the personal-exposed food item, but no mediator for weight loss or binge eating reduction. Stronger habituation of eating desires was not associated with better treatment outcome.

Though participants in the control intervention received an extensive program on improving lifestyle to achieve weight loss, participants in the cue exposure intervention lost significantly more weight at post-measurement, as well as at follow-up. While previous studies have reported prevention of weight regain after weight loss (Mount et al., 1990), and weight loss in a program containing cue exposure elements (Boutelle, Knatz, Carlson, Bergmann, \& Peterson, 2017), this is the first study that found benefits of pure exposure therapy for weight loss. A second important finding is that, although the reduction of general eating psychopathology did not differ between conditions, binge eating frequency strongly decreased in the cue exposure condition. While participants in the exposure condition had a marginally higher binge eating frequency at pre-measurement compared to the control condition, binge eating frequency was reduced to zero in all participants in the exposure condition. Three months after the end of the intervention, participants in the cue exposure condition still had a larger reduction of binge eating frequency compared to control participants. Finding beneficial effects of exposure on binge eating is in line with pilot studies in bulimia nervosa patients (Jansen et al., 1992, 1989; Martinez-mallen et al., 2007; Toro et al., 2003). 
Less kcal consumption of the personal-exposed food item after exposure replicates findings of a previous cue exposure study (Schyns et al., 2016). However, no significant differences were found on kcal consumption of the personal-non-exposed food item, or the general-exposed food item. Regarding the general-exposed food item, a possible explanation is that although the food item was selected as most palatable from a list of ten items, it rarely occurred that participants also mentioned this specific food item during the CS-US interview. In other words, although participants rated these items as palatable; it is possible that these were not their 'problematic' food items in daily life and, therefore, did not lead to important learning experiences. So it seems to be important to always include personalized food items that are associated with a loss of control and not snack foods in general. We did not find generalization; participants did not eat less of the tasty food item they were not exposed to. So even during intensive exposure therapy (eight sessions and an average of 28 homework exposure exercises), participants do not transfer the inhibitory learning experiences to non-exposed food items. Therapists should therefore include as many problematic food items during exposure sessions as possible.

A surprising finding was that we generally failed to induce cue reactivity during the reactivity task at the pre- and post-measurements, which was in sharp contrast with the cue induced eating desires during the exposure sessions. The participants might have suppressed their hedonic responses to food during the measurement sessions because they were in therapy. Recently, attention has been paid to 'mindsets'; palatable versus health mindsets might explain inconsistent findings concerning reward responses to food cues in obese people (Roefs, Houben, \& Werthmann, 2015; Werthmann, Jansen, \& Roefs, 2016). More specifically, certain states/contexts/cues induce a health mindset, for example being in a hospital might facilitate thinking about the health effects of eating the food instead of thinking about its tastiness, thereby decreasing eating desires. Other contexts might induce a hedonic mindset, for example thinking about the delightful taste of chocolate, and strong eating desires. The current 'measurement/treatment context' might have triggered a health mindset, causing cue reactivity responses to be dampened during the measurement sessions.

Habituation of cue reactivity and expectancy violation were investigated as mechanisms of change during therapy. Although eating desires indeed habituated within (WSH) and between (BSH) exposure sessions, no correlations were found between WSH/BSH and significant treatment outcomes, with one exception: WSH did significantly correlate with weight loss at post-measurement, in the direction that more WSH was related to less weight loss. Overall, it can be concluded that larger habituation was not predictive of better treatment outcome, which is a replication of a previous one-session cue exposure study (Schyns et al., 2016) and in line with findings in anxiety exposure studies (Craske et al., 2008). With regard to expectancy violation, it was found that reductions of CS-US 
expectancies after the intervention (post-treatment and follow-up) were stronger in the exposure versus control condition. Expectancy violation was found to be a significant and full mediator between condition and kcal intake of the exposed food item, but it did not mediate weight loss and binge eating reduction and it was not a moderator for any treatment outcome. The finding that expectancy violation was a significant mediator for kcal intake is interesting and in line with previous research (Schyns et al., 2016), suggesting that that the violation of expectancies indeed is an important target for cue exposure sessions, though it's causal role needs to be investigated in experimental designs that manipulate expectancy violation. And although good sleep has shown to be important for consolidation of new CS-noUS memories (e.g., Pace-Schott et al., 2012; Spoormaker et al., 2012), sleep quality did not influence or moderate treatment outcome.

To conclude, cue exposure therapy that targets inhibitory learning is effective in the reduction of body weight, binge eating frequency and snacking behavior of exposed foods in overweight participants. Cue exposure sessions should not aim at habituation of eating desires. Although habituation might occur during the exposures, habituation was not related to better treatment outcome. Instead, the current data suggest that the violation of individual CS-US expectancies should be the target during the cue exposure sessions. 




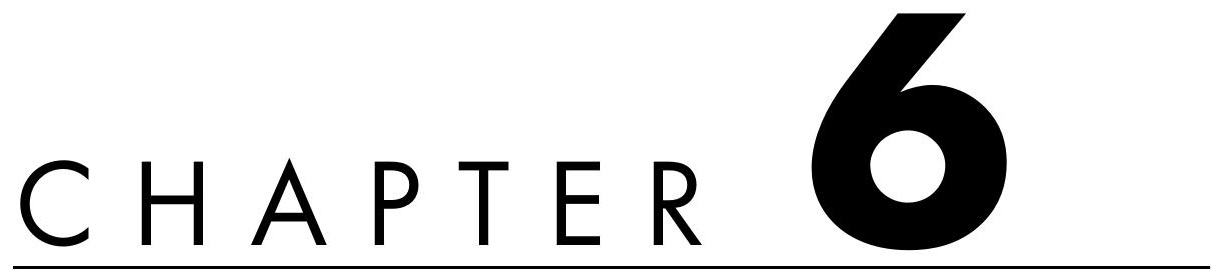

WHAT WORKS BETTER?

FOOD CUE EXPOSURE AIMING

AT THE HABITUATION OF

EATING DESIRES OR FOOD CUE

EXPOSURE AIMING AT THE

VIOLATION OF OVEREATING

EXPECTANCIES?

This chapter is an adapted version of the following published article:

Schyns, G., van den Akker, K., Roefs, A., Hilberath, R., \& Jansen, A. (2018). What works better? Food cue exposure aiming at the habituation of eating desires or food cue exposure aiming at the violation of overeating expectancies? Behaviour Research and Therapy, 102, 1-7. https://doi.org/10.1016/j.brat.2017.12.001 


\section{ABSTRACT}

OBJECTIVE: This study tested the role of habituation of eating desires and violation of CSUS expectancies during food cue exposure in obese women.

METHOD: 52 obese females were randomised into a two-session exposure condition aimed at habituation, a two-session exposure condition aimed at expectancy violation, or a no-treatment control condition. Eating in the absence of hunger, habituation of eating desires, expectancy violation and duration of exposure were measured.

RESULTS: Both exposure conditions ate significantly less of the foods included in cue exposure (i.e., exposed foods) compared to the control condition, though there were no differences between both types of exposure. No differences were found between conditions regarding the eating of non-exposed foods. Changes in eating desires (habituation) and loss of control expectancies (expectancy violation) were no significant mediators for the intake of exposed or non-exposed foods, whereas lower expectancies at the end of the exposure significantly mediated the relationship between condition and less intake of the non-exposed, but not exposed, foods.

CONCLUSIONS: While food cue exposure in obese women led to less eating of exposed foods, the effect did not seem to be driven by habituation of eating desires or expectancy violation. It is discussed why exposure works.

KEYWORDS: exposure therapy; inhibitory learning; habituation; expectancy violation; obesity; eating in the absence of hunger 


\section{INTRODUCTION}

Worldwide, the obesity prevalence has reached dramatic proportions, and the necessity for effective interventions is high (Flegal, Kruszon-Moran, Carroll, Fryar, \& Ogden, 2016). The fact that weight loss interventions that aim at a negative energy balance, by reducing energy intake and increasing energy expenditure, have disappointing effects on successful weight loss fuels the search for effective interventions (e.g., Curioni \& Lourenco, 2005; Franz et al., 2007). A major cause for weight gain is the eating for hedonic purposes instead of physiological needs, also referred to as eating in the absence of hunger (EAH; Kral et al., 2013; Lowe \& Butryn, 2007). EAH usually is promoted by food-related cues; they initiate preparatory processes for food intake, including psychological (e.g., eating desires) and physiological responses (e.g., salivary production; Jansen, 1998). Indeed, this so-called cue reactivity has shown to be higher in overweight versus normal weight individuals (Ferriday \& Brunstrom, 2011), significantly correlated to food intake in overweight individuals (Jansen et al., 2003), prospectively related to weight gain (Boswell \& Kober, 2016), and significantly reduced in successful dieters (who were previously obese) versus unsuccessful dieters (Jansen, Stegerman, Roefs, Nederkoorn, \& Havermans, 2010). Hence, it might be important to tackle cue reactivity in interventions in order to achieve successful weight loss.

Cue reactivity might (at least partly) be learned through classical conditioning (Jansen, 1998; Jansen, Schyns, Bongers, \& van den Akker, 2016): when specific cues, such as the smell or sight of food, become associated with food intake (unconditioned stimulus; US) through repeated pairings, these become conditioned stimuli (CS) for food intake and capable of initiating cue reactivity (conditioned response; $C R$ ). Several human laboratory studies found evidence that associations between food intake (US) and initially neutral stimuli (CS) are easily learned, and that - as a result of this learning process - CSs easily acquire the ability to elicit conditioned eating desires (e.g., Bongers, van den Akker, Havermans, \& Jansen, 2015; van den Akker, Jansen, Frentz, \& Havermans, 2013). Because many eating desires are so easily acquired through classical conditioning, extinction of such associations might be the appropriate way to decrease the learned cue reactivity. Exactly this is the aim of food cue exposure: to expose participants to CSs, such as the smell and sight of food, while food intake (US) is prevented. Doing this repeatedly enables the forming of new associations: the CS does not lead to the US (Bouton, 1993; Bouton \& King, 1983; Jansen, 1998). Exposure therapy is theorized to be most effective when inhibitory learning is maximal; the new CSnoUS association should become stronger than the old CS-US association (Craske et al., 2008). Pilot studies have shown that food cue exposure succefully reduces food cravings and binge eating in bulimia nervosa patients (Jansen, Broekmate, \& Heymans, 1992; Jansen, Van den Hout, De Loof, Zandbergen, \& Griez, 1989; Martinez-mallen et al., 2007; Toro et al., 2003; see also Jansen et al., 2016). Further, studies in overweight adult samples have 
shown that cue exposure is effective in preventing weight regain after successful weight loss (Mount, Neziroglu, \& Taylor, 1990), as well as in diminishing EAH of food items that were specifically included in therapy (i.e., exposed foods; Schyns, Roefs, Mulkens, \& Jansen, 2016)

Although food cue exposure indeed seems beneficial for overeating (Schyns et al., 2016), there is little research available on its working mechanism: why is it effective? According to the influential Emotional processing Theory, exposure sessions should aim at habituation of fear within (WSH) and between (BSH) exposure sessions (Foa \& Kozak, 1986; Foa \& McNally, 1996). However, Craske and colleagues (2008) argue that neither WSH nor BSH in exposure therapy are consistently related to better treatment outcome. This seems to be true for food cue exposure as wel; WSH of cue reactivity was not related to better outcomes (Schyns et al., 2016). These findings may have important clinical implications: therapists should no longer focus on diminishing eating desires during exposure sessions. However, this automatically leads to the question what the alternative focus of cue exposure sessions should be.

Craske and colleagues (2014) argue that one should focus on the explicit violation of CS-US expectancies during exposure (Craske, Treanor, Conway, Zbozinek, \& Vervliet, 2014). Instead of waiting until fear declines during exposure, therapists should carefully introduce CSs (e.g., high heart-rate) that maximize the mismatch between the expectancy of the US to take place (e.g., heart attack) and the actual outcome (e.g., the absence of the heart attack; noUS), thereby strengthening the CS-noUS association. Using this method, exposure sessions can be stopped when the feared outcome is no longer expected, which might take less time than waiting until fear levels habituate. Exposure sessions for panic disorder with agoraphobia focusing on expectancy violation have shown to result in better treatment outcome compared to exposure sessions focusing on habituation (Salkovskis, Hackmann, Wells, Gelder, \& Clark, 2006), and continuing exposure therapy until the expectancy of the feared outcome was $5 \%$ or less was superior to exposure therapy that ended at higher expectancies (Deacon et al., 2013). Expectancy violation during exposure seems well-translatable to food cue exposure, as expectancies of overeating and loss of control can be violated (Jansen et al., 2016; van den Akker, Schyns, \& Jansen, 2016). Indeed, lower expectancies after food cue exposure have found to be related to less EAH of exposed foods (Schyns et al., 2016).

The aim of the present study is to investigate whether focusing food cue exposure on violation of expectancies is more effective to reduce kcal intake of exposed and nonexposed foods than focusing on habituation of eating desires. Two exposure conditions are compared: one exposure condition focuses two sessions on the habituation of eating desires (Exposure focused on Habituation; ExpHAB), and the other exposure condition focuses two sessions on the violation of cue - intake (CS-US) expectancies (Exposure focused 
on Expectancy Violation; ExpEV). Participants are randomly assigned to either exposure condition (ExpHAB or ExpEV) or a no-treatment control condition. It is hypothesized that 1) the ExpEV condition eats less of exposed and non-exposed foods than the ExpHAB condition, while kcal intake in the control condition will be lower compared to both the ExpEV and ExpHAB conditions, and that 2) shorter exposure times are needed for the violation of expectancies (ExpEV) than for the habituation of eating desires (ExpHAB). In addition, 3) stronger habituation of eating desires and violation of CS-US expectancies during exposure sessions are expected to mediate the relationship between condition and intake.

\section{METHODS}

\section{PARTICIPANTS}

Female obese participants $(\mathrm{BMI}>30)$ who are motivated to lose weight, aged 18 to 65 years, were recruited through advertisements. The exclusion criteria were: suffering from self-reported smelling problems (indication of anosmia), pregnancy, currently receiving psychotherapeutic or psychopharmacological treatment, and bariatric surgery (pre and post-operative). Participants were randomly assigned to the ExpHAB condition $(n=17)$, the ExpEV condition $(n=18)$, or the control condition $(n=17)$. No participants dropped out during the study. The study was approved by the Ethical Committee of the Faculty of Psychology and Neuroscience of Maastricht University.

\section{PROCEDURE}

Two exposure sessions were planned on two separate days within one workweek. Prior to session one, the participant was instructed to buy four highly palatable snack foods which were perceived as their 'favourite foods' and difficult to refrain from. The participants was instructed to bring three normal-sized packages of each food item to the first session. To standardize hunger, the participant was also instructed to consume a small meal 2 hours prior to each appointment.

A summary of the timing of the main study assessments and design is displayed in Figure 1. After arrival in the laboratory on day 1, the participant immediately handed in the food items and gave informed consent, followed by rating baseline hunger and desire to eat. In order to induce a state of absence of hunger during food cue exposure, the participant received a prepacked cup of yoghurt and muesli in a flavour of choice (neutral, honey, strawberry, peach, berries; $\sim 170 \mathrm{~g}, \sim 233 \mathrm{kcal}$ ). If a participant was lactose-intolerant, she received two muesli bars in a flavour of choice (neutral, cranberry, hazelnut, golden syrup; 2 
bars: $\sim 54 \mathrm{~g}, \sim 231 \mathrm{kcal})$. The participants was instructed to finish the entire cup or both bars, and to fill out a questionnaire about its taste and quality, followed by 15 minutes of satiation time. After these 15 minutes, the participant again rated hunger, as well as tailored foodspecific CS-US expectancies and the palatability of the food items. Then, the intervention started at this point: both exposure conditions (ExpEV and ExpHAB) received food cue exposure for at least 10 and maximally 40 minutes, dependent on whether the target of the specific intervention (habituation or expectancy violation) was reached (see 2.3). On the second day, the participant started with the measurement of baseline hunger and eating desire, followed by the muesli snack and 15 minutes of satiation. After 15 minutes, hunger was rated and the second food cue exposure session took place. After the exposure in session two, the participant received another muesli snack to ensure that the participant remained in the absence of hunger during test (though the participant was allowed to not finish the second muesli snack if it was too much), once more followed by 15 minutes of satiation time and ratings of hunger and eating desires. The participant then rated the food-specific CS-US expectancies which was followed by the bogus taste test. Thereafter, the abstinence check of food items was completed and the participant's weight and height was measured.

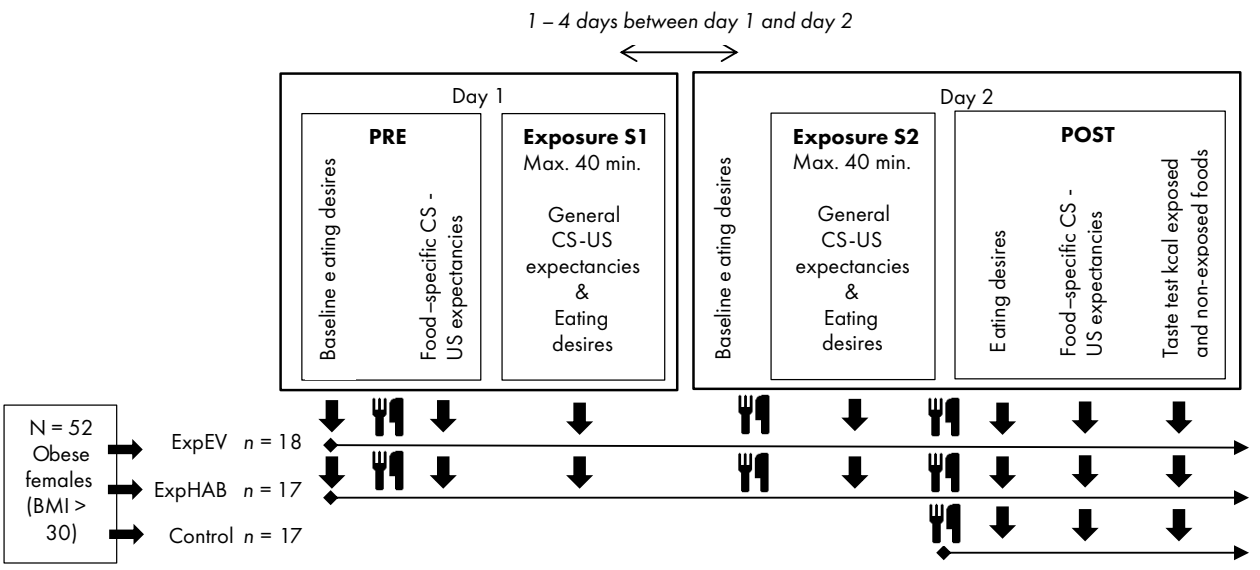

Figure 1. Overview of main study measurements during days 1 and 2 including exposure sessions 1 and 2 (S1 and S2), separately for the control condition and both exposure conditions (ExpEV and ExpHAB). The symbol $\boldsymbol{\Downarrow}$ reflects measurements as displayed in the upper text, while the symbol $\mathbf{W}$ reflects the muesli snack which was always followed by 15 minutes of satiation time in order to induce a state of absence of hunger

Participants in the control condition followed the only the second part of the program on day two, in which each participant received a muesli snack (including satiation time and hunger rating) and completed food-specific CS-US expectancies, current desire to eat, the bogus taste test, abstinence and palatability check, and the measurement of weight 
and height. Participants in the exposure condition were instructed on the first day that they were not allowed to eat the four food items until the appointment on day 2 was completed; participants in the control condition received this instruction one to five days before the first appointment. Participants received $€ 50$,- for participation and a refund for the purchased foods. To keep recruitment advertisements similar for all conditions (i.e., receiving a training), the control condition received two sessions of food cue exposure after all measurements were done (without data-collection during the exposure).

\section{EXPOSURE INTERVENTION}

The participant was exposed to large portions of two of four favourite foods, which were put in bowls which were continuously available right in front of the participant on a tray. Foods were randomly allocated as exposed or non-exposed foods. The participant was instructed to smell, touch, lick the foods and to take a tiny bite. Both exposure conditions received the same rationale to the exposure exercises, which was largely descriptive in nature, followed by a condition-specific verbal script during exposure, see Table 1. Eating desires and CSUS expectancies were measured at condition-specific time-points and the length of the exposure session was determined by the $50 \%$ reduction target (see 2.4.1 and 2.4.2). At the end of each session, the participant was instructed to throw away all food in a garbage bin.

\section{EXPOSURE AIMED AT HABITUATION (EXPHAB)}

The therapist followed the verbal script as displayed in Table 1. Eating desires were measured before the start of the exposure, when food was already visible, and every five minutes during exposure. In addition, general CS-US expectancies were measured at the start, at the peak of eating desires, and at the end of the session. Exposure sessions in this condition were terminated when eating desires were reduced by $50 \%$ relative to the peak desire, or when 40 minutes of exposure were reached.

\section{EXPOSURE AIMED AT EXPECTANCY VIOLATION (EXPEV)}

The therapist followed the verbal script in Table 1. General CS-US expectancies were measured before the start of the exposure, when food was visible, and every five minutes during exposure. In addition, eating desires were measured at the start, at the peak of CS-US expectancies, and at the end of the session. Exposure sessions in this condition were terminated when CS-US expectancies were reduced by $50 \%$ relative to the peak expectancies, or when 40 minutes of exposure were reached. 
Table 1. Verbal script during exposure for the exposure habituation (ExpHAB) and exposure expectancy violation (ExpEV) conditions.

\section{Rationale}

We are going to do exposure exercises; this means that we will investigate what happens when we see, touch, lick and taste this food, but wait to actually eat the food. Eating desires can become high during these exercises; we are going to examine how this works for you, and how this develops over time. For this investigation, I will occasionally ask you to fill in a short questionnaire.

\section{ExpHAB}

1. Smell the entire bowl and take one piece of food in hands to smell

- How does the smell affect your eating desires?

- How does holding the food in your hand affect your eating desires?

2. Lick the food

- How does licking the food in your hand affect your eating desires?

3. Take a tiny bite

- How does tasting the food affect your eating desires?

- How does chewing the food affect your eating General: desires?

- At what level are your eating desires right now?

- Seeing the food right now, how does this affect your eating desires?

\section{ExpEV}

1. Smell the entire bowl and take one piece of food in hands to smell

- How does the smell affect you, how hard is it to not eat the food?

- How does holding the food in your hand affect you, how hard is it to not eat the food?

2. Do your think that licking the food will make it harder to refrain from eating the food/ will cause you to lose control? Let's test it out.

- What happened? Why did you not lose control?

- Do you find it hard not to eat the food after licking the food?

3. Do your think taking a tiny bite will make it harder to refrain from eating the food/ will cause you to lose control? Let's test it out.

- What happened? Why did you not lose control?

- It is hard to stop eating after this bite?

- It is hard to stop eating after chewing the food?

- How it is possible that you are able to stop General: eating?

- Seeing the food right now, how hard is it to not eat the food?

- Why are you not losing control?

End of exposure:

- What did you learn from these exercises?

1. Both conditions received the same rationale.

\section{ASSESSMENT}

\section{HABITUATION OF EATING DESIRES}

Desire to eat was measured on a VAS ('How strongly do you desire palatable food right now?'), ranging from 0 (no desire at all) to 100 (very strong desire).

\section{EXPECTANCY VIOLATION}

Two general CS-US expectancy items were rated during the exposure sessions on the expectancy of a CS to be followed by the US using 100mm VAS ('How strongly do you believe in the following thought right now?'), ranging from 0 (not at all) to 100 (very strongly): 'If palatable food is in front of me, then I cannot refrain from eating it' and 'If I eat 
a small amount of palatable food, then I cannot stop eating. Further, both expectancy items were adapted to each of four (exposed and non-exposed) food items: 'If [food item] is in front of me, then I cannot refrain from eating it' and 'If I eat a small amount of [food item], then I cannot stop eating, resulting in eight tailored food-specific CS-US expectancy items.

\section{DURATION OF EXPOSURE}

Each exposure session stopped when habituation or expectancy violation had occurred $(>=$ $50 \%$ reduction of desires vs. expectancies). The number of exposure minutes was recorded for each session.

\section{EAH}

The EAH paradigm was adapted from Birch and Fisher (2000). To induce a state of satiation, the participant received a muesli yoghurt or muesli bars (see 2.2). 15 minutes after eating the muesli snack, current hunger was measured on a VAS ('How hungry are you right now?'), ranging from 0 (not hungry at all) to 100 (very hungry). Then, a 10-minute bogus taste test started during which the participant was told that this test was to assess taste perception of the four different foods that were individually selected by the participant. The participant was instructed to rate the taste of each of the food items (using a questionnaire) while being allowed to eat as much as she wanted from the generous portions of each food item. After the taste test, foods were removed. Each food item was weighed before and after the test, and the number of consumed kcal was calculated and averaged for the exposed vs. the non-exposed foods.

\section{BMI}

Height and weight of the participant was measured to calculate BMI $\left(\mathrm{kg} / \mathrm{m}^{2}\right)$.

\section{PALATABILITY CHECK}

To control for the palatability of the four food items used in the study, palatability was measured using visual analogue scales (VAS; 'How tasty is [food item] for you?', ranging from 0 (not tasty at all) to 100 (very tasty).

\section{ABSTINENCE CHECK}

The participant rated the number of eating occasions on which they consumed the four food items that were used in the study, since receiving the instruction of not consuming these items (i.e., on the first appointment in the ExpHAB and ExpEV condition, or before the first appointment in the control condition), from 0 (not at all) to 6 (six times or more). In addition, the number of days between the instruction and the taste test were recorded. 


\section{STATISTICAL ANALYSES}

For comparisons between the three conditions (ExpHAB, ExpEV, control) on BMI, age, hunger, abstinence and palatability check, post-intervention eating desires and CS-US expectancies, and kcal eaten of the exposed and non-exposed foods, one-way ANOVA's were conducted. Analyses on the comparison of both exposure conditions (ExpHAB and ExpEV) included independent samples t-tests and mixed model ANOVAs for WS variables. As a manipulation check, it was checked whether the percentage reduction of eating desires relative to the peak desire during exposure sessies was stronger in the ExpHAB condition than in the ExpEV condition, and whether the percentage reduction of CS-US expectancies relative to the peak expectancy during exposure sessies was stronger in the ExpEV condition than in the ExpHAB condition. WSH was calculated by subtracting the end-level of desire from the peak desire during each session and averaging the scores for both sessions. BSH was calculated by subtracting the peak desire from session two from the peak desire of session one (Craske et al., 2008). Expectancy violation was calculated by change scores from peak CS-US expectancy minus end CS-US expectancy per session, and averaged over sessions. To check whether hunger levels during exposure were different from hunger levels during the taste test, a paired samples t-test was used. Since separate CS-US expectancies and consumed kcal for exposed and non-exposed foods could not be calculated for the control condition, two (identical) average scores of all food items were used in this condition. Mediation effects of habituation of eating desires and absolute post-intervention eating desires, as well as violation of expectancies and absolute postintervention CS-US expectancies were tested by applying the bootstrapping method with a 95\% confidence interval of the indirect effect using 5000 samples as described by Preacher and Hayes (2008). Indirect mediation effects are considered significant when the 95\% confidence interval does not contain zero, and can be assessed even in the absence of a total effect (i.e., the effect of X on Y [in current study: condition on kcal intake]; e.g. Preacher \& Hayes, 2004; Zhao, Lynch, \& Chen, 2010).

\section{RESULTS}

\section{PARTICIPANTS}

The participants' mean BMI was not significantly different between the ExpHAB condition $(M=34.68, S D=3.46)$, the ExpEV condition $(M=34.95, S D=2.79)$, and the control condition $(M=36.14, S D=4.41), F(2,51)=0.79, p=.460, \eta_{p}^{2}=0.03$. This also held for age, with a mean age of 42.06 years $(S D=8.99)$ in the ExpHAB condition, 46.67 years $(S D=$ $6.05)$ in the ExpEV condition, and $45.94(S D=8.58)$ in the control condition, $F(2,51)=$ $1.68, p=.197, \eta_{p}^{2}=0.06$. 


\section{MANIPULATION CHECKS}

\section{EXPERIMENTAL MANIPULATION OF EATING DESIRES AND EXPECTANCY VIOLATION DURING FOOD CUE EXPOSURE}

Average peak and end-level eating desires and CS-US expectancies are shown in Figure 2. The average percentage reduction in eating desires over two sessions in the ExpHAB condition $(M=-39.57, S D=29.93)$ was not significantly different from the ExpEV conditions $(M=-37.35, S D=29.53), t(33)=0.22, p=.827, d=0.08$. In contrast, the general CS-US expectancy percentage reduction in the ExpEV condition $(M=-61.96, S D=31.63)$ was significantly larger than the reduction in the ExpHAB condition $(M=-35.40, S D=$ 29.27), $t(33)=2.57, p=.015, d=0.87$.

\section{ABSTINENCE CHECK}

The number of days of during which participants were instructed to remain abstinent of the four food items before the taste test was not significantly different between conditions (ExpHAB: $M=1.76, S D=0.97$; ExpEV: $M=2.22, S D=0.94$; Control: $M=2.12, S D=1.17$ ), $F(2,49)=0.94, p=.398, \eta_{p}^{2}=.04$. Consumption of the four favourite food items between conditions, against the instruction, was not significantly different between conditions (ExpHAB: $M=0.03, S D=0.08$; ExpEV: $M=0.01, S D=0.06$; Control: $M=0.11, S D=0.24$ ), $F(2,49)=2.50, p=.093, \eta_{p}^{2}=.09$.

\section{PALATABILITY CHECK}

Average palatability ratings of the exposed foods (ExpHAB: $M=86.44, S D=17.50$; ExpEV: $M=84.64, S D=11.42$; Control: $M=86.72, S D=11.90$ ) and non-exposed foods (ExpHAB: $M=85.43, S D=12.15$; ExpEV: $M=86.42, S D=13.43$; Control: $M=86.72, S D=11.90$ ) were equally high and similar in all conditions, as reflected by the absence of significant main effects of condition, $F(2,49)=.05, p=.954, \eta_{p}^{2}<0.01$, food item (exposed versus nonexposed), $F(1,49)=.02, p=.884, \eta_{p}^{2}<0.01$, and an absent condition ${ }^{\star}$ food item interaction effect, $F(2,49)=0.22, p=.803, \eta_{p}^{2}<0.01$.

\section{HUNGER RATINGS}

Although average hunger ratings before starting both exposure sessions were low $(M=$ $19.23, S D=17.83)$, indicating absence of hunger, hunger levels before starting the taste test were significantly lower $(M=13.54, S D=17.04), t(34)=2.54, p=.016, d=0.43$. When comparing hunger before the taste test in all three conditions, a significant difference was found (ExpHAB: $M=15.29, S D=14.36$; $\operatorname{ExpEV} ; M=11.89, S D=19.50$; Control: $M=$ $29.00, S D=25.22), F(2,49)=3.48, p=.039, \eta_{p}^{2}=.12$. Post-hoc tests reveal that hunger was significantly higher in the control condition than the ExpEV condition $(p=.016)$, 


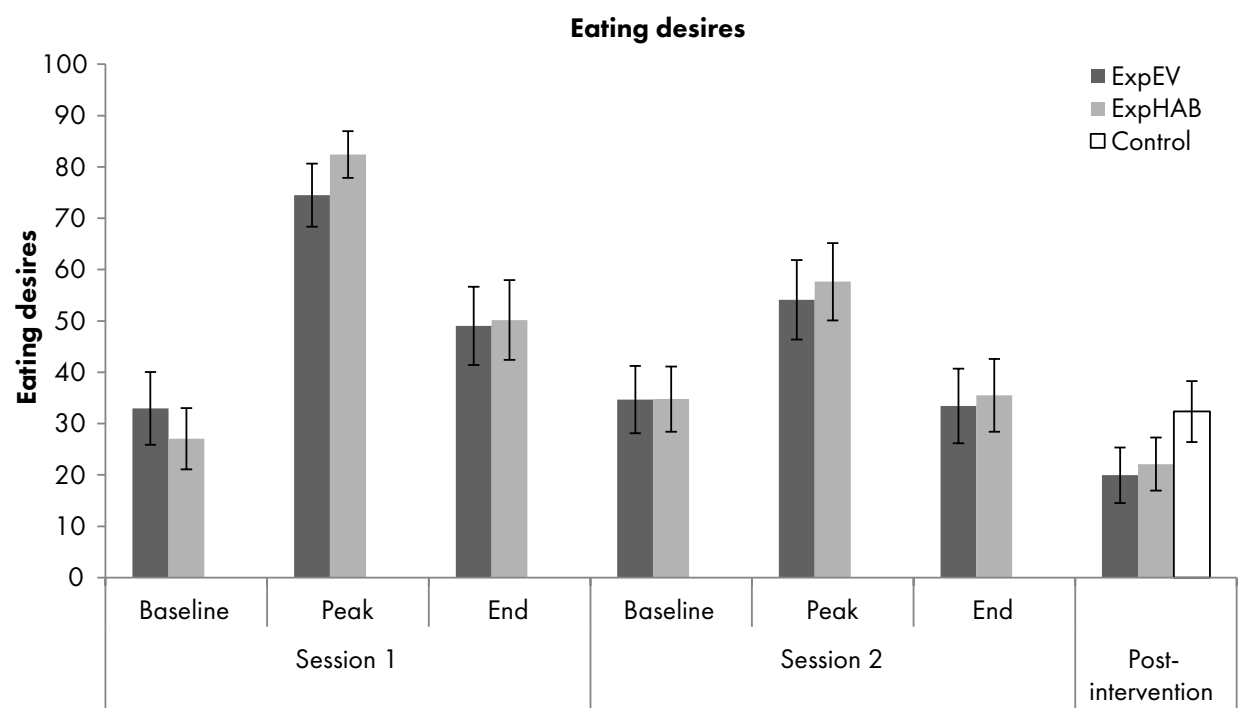

CS-US expectancies

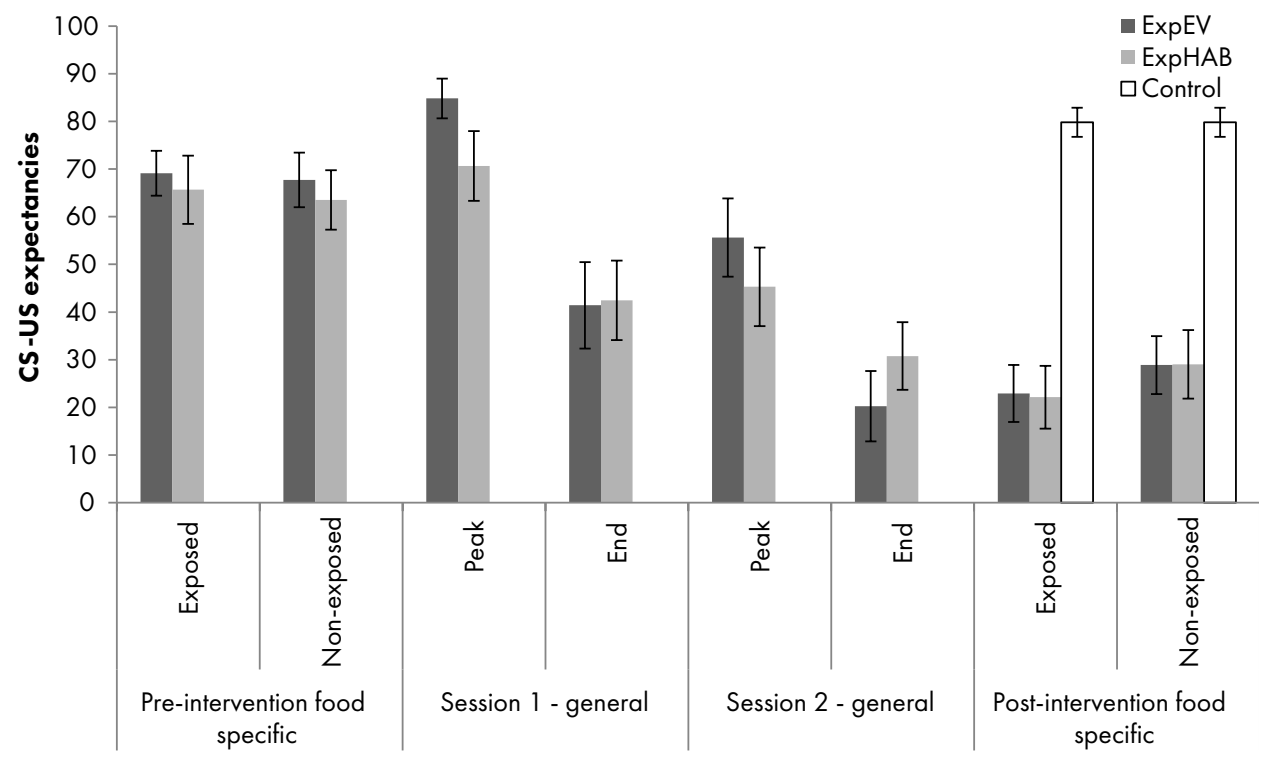

Figure 2. Average eating desires (top) and CS-US expectancies (bottom) during the sessions and pre- and post-intervention, separated by condition (ExpEV: Exposure Expectancy Violation; ExpHAB: Exposure Habituation; Control). Error bars represent standard errors of means. 
and marginally higher in the control condition than the ExpHAB condition $(p=.053)$, while differences were not significantly different between both exposure conditions $(p=$ .620). Including hunger as a covariate was however not a significant contributor for any of the outcome measures, nor was the interaction between condition and hunger significant for any of the outcome measures. Hunger was therefore not included in the analyses below.

\section{HYPOTHESIS 1) THE EXPEV CONDITION EATS LESS OF EXPOSED AND NON-EXPOSED FOODS THAN THE EXPHAB CONDITION, WHILE KCAL INTAKE IN THE CONTROL CONDITION WILL BE LOWER COMPARED TO BOTH EXPEV AND EXPHAB}

\section{EAH: KCAL INTAKE OF EXPOSED FOODS}

As can be seen in Figure 3, kcal intake of the exposed foods was significantly different between conditions, $F(2,51)=5.24, p=.009, \eta_{p}^{2}=.18$. Post-hoc tests reveal that the ExpEV condition consumed significantly fewer kcal than the control condition $(p=.005)$, as did the ExpHAB condition compared to the control condition $(p=.011)$. However, the kcal consumption was not significantly different between both exposure conditions (ExpEV vs. $\operatorname{ExpHAB} ; p=.781)$.

\section{EAH: KCAL INTAKE OF NON-EXPOSED FOODS}

Also shown in Figure 3, kcal intake of the non-exposed foods was not significantly different between conditions, $F(2,51)=0.04, p=.965, \eta_{p}^{2}<.01$.

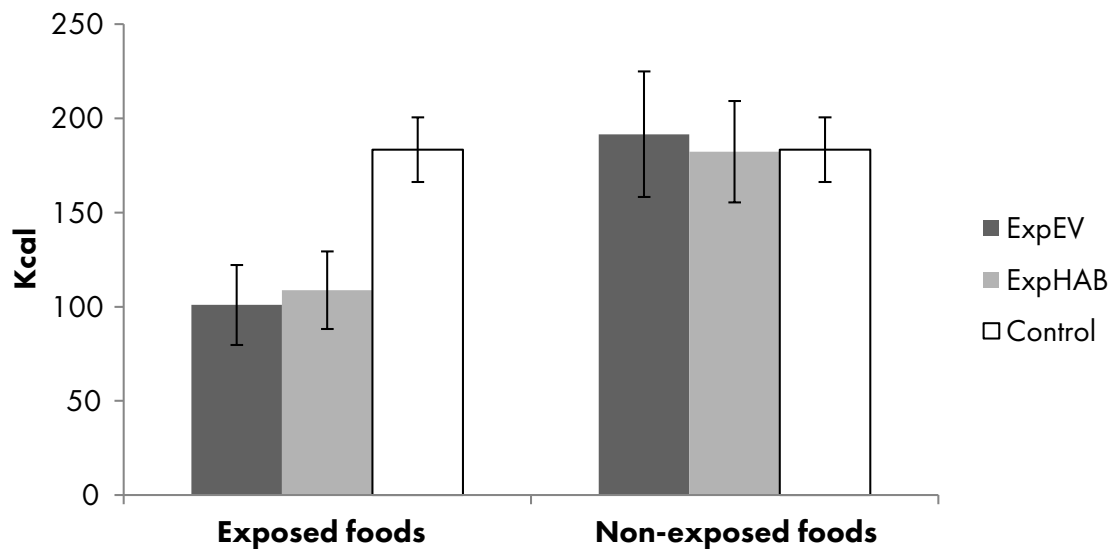

Figure 3. Kcal intake of exposed and non-exposed food items, separated by condition (ExpEV: Exposure Expectancy Violation; ExpHAB: Exposure Habituation; Control). Error bars represent standard error of means. 


\section{HYPOTHESIS 2) SHORTER EXPOSURE TIMES ARE NEEDED FOR THE VIOLATION OF EXPECTANCIES (EXPEV) THAN FOR THE HABITUATION OF EATING DESIRES (EXPHAB)}

17 of 35 (49 \%; ExpHAB 53 \%; ExpEV 44\%) of participants receiving cue exposure did not reach the end-of-session target in session one within 40 minutes, and used the entire 40 minutes of exposure. In session two, 10 of 35 (29 \%; ExpHAB 41\%; ExpEV 17\%) participants did not reach the end-of-session target within 40 minutes. The number of minutes of exposure was larger in session one (ExpHAB: $M=30.00, S D=12.25$; ExpEV: $M=28.06, S D=12.26$ ) than in session two (ExpHAB: $M=25.88, S D=13.72$; ExpEV: $M=$ $19.17, S D=11.54)$, but similar for both exposure conditions, as reflected by a main effect of session, $F(1,33)=12.08, p=.001, \eta_{p}^{2}=.27$, and no main effect of condition, $F(1,33)=$ $1.32, p=.259, \eta_{p}^{2}=.05$, or a session* ${ }^{\star}$ ondition interaction, $F(1,33)=1.63, p=.211, \eta_{p}^{2}=.05$.

\section{HYPOTHESIS 3) STRONGER HABITUATION OF EATING DESIRES AND VIOLATION OF CS-US EXPECTANCIES DURING EXPOSURE SESSIONS ARE EXPECTED TO MEDIATE THE RELATIONSHIP BETWEEN CONDITION AND INTAKE}

\section{HABITUATION OF EATING DESIRES}

Baseline eating desires were not significantly different between conditions on either exposure session ( $p$ 's > .531). WSH (ExpHAB: $M=27.18, S D=20.00$; ExpEV: $M=23.06$, $S D=19.04$ ) and BSH of eating desires (ExpHAB: $M=24.76, S D=33.90$; ExpEV: $M=20.39$, $S D=41.04)$ were not significantly different between conditions, $t(33)=0.62, p=.537, d=$ 0.22 , and $t(33)=0.34, p=.734, d=0.12$, respectively, indicating that habituation was as strong in both exposure conditions.

Habituation was not found to be a significant mediator between condition and kcal intake. Specifically, WSH and BSH were no significant mediators for kcal intake of exposed foods, $\beta=-0.59,95 \% C I[-5.65,29.34]$ and $\beta=1.76,95 \% C I[-9.20,12.64]$, respectively, and also not for kcal intake of non-exposed foods, $\beta=-4.17,95 \% C I[-40.61,11.42]$ and $\beta=1.08$, 95\% CI [-17.28, 9.05], respectively. Post-intervention eating desires were also no significant mediator for exposed foods, $\beta=.33,95 \% C I[-14.86,13.53]$, or non-exposed foods, $\beta=$ $-2.80,95 \%$ CI [-29.49, 9.35].

\section{EXPECTANCY VIOLATION}

Expectancy violation, measured by the average reduction of general CS-US expectancies during both exposure sessions, was significantly stronger in the ExpEV condition $(M=$ $39.39, S D=24.50)$ than in the ExpHAB condition $(M=21.35, S D=19.99), t(33)=2.38, p$ 
$=.023, d=0.81$ (see Figure 2). The average reduction of food-specific CS-US expectancies from pre to post-measurement did however not differ significantly between the ExpEV and ExpHAB conditions, $F(1,33)=0.12, p=.731, \eta_{p}{ }^{2}<.01$. From pre to post-intervention, the average reduction of expectancies was stronger for the exposed foods compared to the non-exposed foods, $F(1,33)=8.66, p=.006, \eta_{p}^{2}=.21$. The condition ${ }^{\star}$ food item interaction was however not significant, $F(1,33)=0.09, p=.766, \eta_{p}^{2}<.01$, indicating that both exposure conditions had similar CS-US expectancy reductions for exposed and non-exposed foods. When comparing absolute post-intervention CS-US expectancies between all conditions (including the control condition), differences were significant for exposed foods, $F(2,49)=$ $36.19, p<.001, \eta_{p}^{2}=.60$, with post-hoc tests indicating significantly higher expectancies in the control condition vs. both exposure conditions ( $p$ 's $<.001$ ), while no differences between both exposure conditions emerged $(p=.916)$. The same finding held for the expectancies of non-exposed foods: the three conditions differed significantly, $F(2,49)=25.89, p<.001$, $\eta_{p}^{2}=.51$. Expectancies were significantly higher in the control condition vs. both exposure conditions $(p$ 's $<.001)$, while no differences between both exposure conditions were found $(p=.985)$.

Expectancy violation (i.e., the reduction of general CS-US expectancies in the exposure conditions), as well as abolsute post-intervention CS-US expectancies of all condions (including the control condition) were investigated as mediators between condition and kcal intake of exposed and non-exposed foods. Expectancy violation was no significant mediator between the exposure conditions and kcal intake of exposed foods, $\beta=-6.72,95 \%$ $C I[-42.15,17.51]$, or kcal intake of non-exposed foods, $\beta=4.54,95 \%$ CI [-28.50, 54.63]. Post-intervention CS-US expectancies regarding non-exposed foods were found to be a significant mediator between condition and kcal intake of non-exposed foods, $\beta=70.91$, $95 \%$ CI $[9.34,148,46]$. In this model, condition was significantly positively associated with CS-US expectancies, $\beta=50.85, t(50)=7.27, p<.001$ (a-path), indicating that the control condition had higher expectancies than the ExpHAB and ExpEV conditons, while CS-US expectancies were also significantly positively associated with kcal intake, $\beta=1.41, t(50)$ $=2.20, p=.032$ (b-path), indicating that higher CS-US expectancies were associated with eating more kcal. Interestingly, the significant indirect effect was found in the absence of a significant direct effect of condition on kcal intake (of non-exposed foods), $\beta=3.73, t(50)$ $=0.11, p=.91$ (c-path), indicating an indirect-only (full) mediation effect (Zhao et al., 2010). In contrast to the mediating role of post-intervention CS-US expectancies of nonexposed foods, post-intervention CS-US expectancies of exposed foods were no significant mediator between condition and kcal intake of exposed foods, $\beta=35.49,95 \%$ CI [-29.94, 101.18]. 


\section{ASSOCIATIONS BETWEEN HABITUATION OF EATING DESIRES AND EXPECTANCY VIOLATION}

WSH was highly correlated to expectancy violation, $r(35)=.61, p<.001$, while BSH did not significantly correlate with expectancy violation, $r(35)=-.24, p=.171$. Clearly, the reduction of eating desires during exposure sessions coincides with the violation of expectancies.

Summarizing, it was found that both exposure conditions consumed significantly less $\mathrm{kcal}$ of exposed foods compared to the control condition, while no differences were found between both exposure conditions (ExpEV and ExpHAB) on kcal of exposed foods, and no differences were found between conditions were between conditions on kcal non-exposed foods (hypothesis 1). Further, the number of minutes of exposure were not significantly different between exposure aimed at violation of expectancies (ExpEV) and exposure aimed at habituation of eating desires (ExpHAB; hypothesis 2). Lastly, neither the habituation of eating desires nor the absolute eating desires at post-intervention significantly mediated the relationship between condition and kcal intake. While expectancy violation was also no mediatior between condition and kcal intake, absolute post-intervention CS-US expectancies were a significant mediator of non-exposed foods, but not of exposed foods.

\section{DISCUSSION}

The aim of the present study was to investigate whether aiming food cue exposure at expectancy violation is more effective than aiming sessions at habituation of eating desires. It was found that both exposure aimed at habituation of eating desires (ExpHAB) and exposure aimed at expectancy violation (ExpEV) led to less intake of exposed foods compared to intake in the control condition. However, the hypothesised difference between the ExpEV and ExpHAB condition was not found. Both exposure conditions, whether habituation of eating desires or violation of expectancies was aimed at, had a decreased intake compared to a non-exposure control condition. No significant differences were found between the conditions on the intake of non-exposed foods, indicating that exposure did not lead to generalisation of the exposure effect (in neither exposure condition). In addition, the duration of exposure was not different between the ExpEV and ExpHAB conditions. Also contrasting the a-priori hypothesis, neither expectancy violation nor habituation of eating desires were significant mediators between the condition - kcal intake relationship. However, post-intervention CS-US expectancies fully mediated the relationship between condition and kcal intake of non-exposed foods - higher expectancies leading to higher intake - though this relationship was not found for kcal intake of exposed foods. Postintervention eating desires also did not mediate the condition - kcal intake relationship. 
The finding that the intake of exposed foods was significantly less for the exposure conditions compared to a no-treatment control condition, with a large effect size, whereas no generalisation to non-exposed foods was found, replicated findings of a previous study with an active control condition (Schyns et al., 2016). These findings confirm that cue exposure therapy is indeed effective to reduce the overeating of the specific snack foods the person was exposed to, but the effect does not generalise to other favourite snack foods.

Considering the working mechanism of food cue exposure, habituation occurred in both conditions. Thus, without focusing attention to the reduction of desires in the ExpEV condition, desires reduced in this 'cognitive' condition as well. So focusing on the violation of loss of control expectancies while being exposed to one's favourite foods, will also lead to a decrease of eating desires. The other way around, it was demonstrated that expectancies were significantly stronger violated in the condition that explicitly challenged the expectations during the exposure compared to the pure habituation condition, while the change of expectancies from pre- to post-exposure was as strong in both conditions. So the conclusion is that even though more inhibitory learning occurs during exposure sessions when explicitly targeting eating expectancies, the reduction of CS-US expectancies after finishing exposure sessions is as strong as compared to exposure sessions aiming at habituation. Clearly, confirmed by the high correlations between habituation within exposure sessions and expectancy violation, these mechanisms cannot be disentangled: habituation of desires violates expectancies and violated expectancies decrease eating desires. This process was not related to intake: WSH, BSH or expectancy violation during exposure sessions were not found to be significant mediators between conditions and intake of exposed foods or non-exposed foods, so neither the habituation of eating desires nor the violation of expectancies during exposure were nessecary for better outcomes. This finding also held for absolute eating desires, suggesting that eating desires (neither absolute nor reduction) are no important target for exposure sessions. In contrast, the absolute CS-US expectancies were important for the kcal intake of non-exposed foods, but not for exposed foods. It seems that exposure effects with specific foods do translate directly to overeating of these foods, or might go via yet unexplored mechanisms, while for the generalisation of cue exposure, the cognitive translation of inhibitory learning to non-exposed foods is important.

Altogether, these results demonstrate that food cue exposure is a successful intervention to reduce the intake of favourite foods. This study contributes that the focus of cue exposure sessions does not matter; whether the exposure is directed at habituation or expectancy violation, both are successful in decreasing desires, expectancies and food intake. However, achieving lower CS-US expectancies might be important for generalisation of exposure effects. Post-intervention CS-US expectancies have also been associated with less intake after food cue exposure in previous research, though this was found for exposed foods 
while this association was not investigated for non-exposed foods (Schyns et al., 2016). Our findings contrast the recommendations made by Craske et al. (2014), who propose that exposure sessions aimed at violation of expectancies yield better treatment outcomes than exposure sessions aimed at habituation. However, the current findings do fit with an exposure study in spider phobics in which exposure explicitly aimed at the challenging of dysfunctional cognitions led to similar treatment effects and cognitive alterations compared to an exposure condition that was purely behavioral (Raes, Koster, Loeys, \& De Raedt, 2011). Still, in the spider phobics study, cognitive change was a mediator for treatment outcome, whereas a change in expectancies was not associated with intake in the present study, though the absolute belief in the expectancies was (less belief associated with less intake).

To conclude, while two sessions of food cue exposure in obese women led to less intake of exposed foods, specifically aiming exposure at violation of expectancies did not to less intake than aiming exposure at habituation of eating desires. The strong positive correlation between the reduction of eating desires and the reduction of CS-US expectancies during sessions shows that expectancy reduction and desire reduction go hand in hand. Although it remains important to pinpoint the working mechanism of cue exposure interventions, the current data do have an optimistic message for clinical practice: doing cue exposure, regardless of its 'special' focus, is effective in the decrease of food desires and food intake.

\section{CONFLICT OF INTEREST}

The authors declare no conflict of interest. 




\section{CHAPTER}

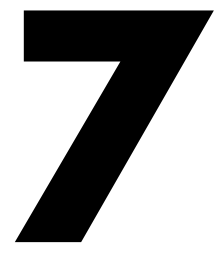

\section{THE POWER CHAPTER}



The results of the four studies in this dissertation show relatively consistent findings regarding the effects of food cue exposure on overeating during a bogus taste test: all studies found that participants who received cue exposure consumed less kcal of exposed foods than participants in a control condition, while generalisation of cue exposure effects to nonexposed foods was not found in three of four studies. When investigating cue exposure's working mechanisms, attention was paid to habituation of cue reactivity during and between exposure sessions in each of the studies, as well as to the violation of overeating (CS-US) expectancies. Regarding habituation of cue reactivity, the overall finding was that habituation was not related to intake of foods during the taste test. Findings on expectancy violation were more mixed, violation of expectancies during exposure (i.e., change in expectancies) was found to be significantly associated with intake in chapter 5 , while this association was not significant in chapters 3 and 6 , and not investigated in chapter 2. Postintervention overeating expectancies were significantly correlated to intake in chapters 2 and 6, but not investigated in chapters 3 and 5. Lack of power might be a problem in these studies with modest samples sizes. Since the studies were comparable in design, the data of all studies were aggregated into one dataset to overcome power issues. Using a larger dataset of the participants of all four studies combined, it is investigated whether cue exposure effects on intake of exposed foods do generalise to non-exposed foods. In addition, associations between habituation of cue reactivity, expectancy violation and kcal intake were investigated.

In total, 176 participants were included in the present investigation (cue exposure $n$ $=96$, control $n=80)$ with an average age of $37.42(S D=15.42)$ years, including 16 males and 160 females. 148 participants (84.1\%) sufficed the (for children adjusted) criterion of obesity (BMI > 30), the remainder $28(15.9 \%)$ participants were overweight (BMI $>25)$. Of the 96 participants receiving food cue exposure, 21 participants received one cue exposure session (chapter 2), 56 participants received two sessions (chapters 3 and $6^{1}$ ) and 19 participants received eight sessions of cue exposure (chapter 5). Although all participants received a bogus taste test after cue exposure vs. control, the design of the taste tests varied slightly between studies. ${ }^{2}$ To compare the intake during the taste test in all studies, $z$-scores were calculated for exposed and non-exposed foods in each study. Regarding habituation

1 In chapter 6, participants were randomly assigned to either a cue exposure condition targeting habituation of eating desires or a cue exposure condition targeting expectancy violation. For the present investigation, all participants in both cue exposure conditions were included in the food cue exposure sample.

2 The number of different foods (range $2-6$ different food items) presented to the participant was different between studies. In addition, while general exposed and non-exposed foods were compared between conditions in the taste tests described in chapter 2 and 3, foods in chapters 5 and 6 were personally selected. In chapter 5, one general exposed food item was additionally included during the taste test, but no general non-exposed food item was included as comparison and therefore not included in the present investigation. Another important difference is that pure kcals were compared in chapters 2, 5 and 6, while the intake in chapter 3 was converted into a percentage of daily required energy intake for children. 
of cue reactivity, eating desires were identically measured in all studies by using $100 \mathrm{~mm}$ Visual Analogue Scales (VAS), from 0 (no desire to eat) to 100 (an extreme desire to eat). Within session habituation was calculated by subtracting the end eating desire from the session's peak eating desire, and, in case of more than one exposure session, calculating the average over sessions. As chapters 3, 5 and 6 included more than one exposure session, between session habituation was also calculated by subtracting peak desires of the final session from peak desires in the first session. Salivation as a measure of cue reactivity was only measured in two of four studies (chapters 2 and 3) and therefore not included in the present investigation. CS-US expectancies were measured in all studies before and after cue exposure, using 'If CS, then US' statements. Variations of the statements 'If I have tasty food in front of me, then I cannot resist to eat it' and 'If I eat a small amount of tasty food, then I cannot stop eating' were used in all studies, while also personalized 'If CS then US' statements were used in chapter 5. Expectancies were measured on a $100 \mathrm{~mm}$ VAS, with higher scores reflecting higher overeating expectancies, in chapters 2, 5 and 6 . The average of all expectancy items on pre and post-intervention was used for the present investigation, as well as change scores as a measure of expectancy violation (pre minus postmeasurement). In the cue exposure study with children in chapter 3, a 5-point Likert scale was used to facilitate the rating of expectancies. In order to compare these expectancies with the 100-point scale expectancies of other studies, data were transformed. ${ }^{3}$ Results below are presented with and without the data of chapter 3.

Results showed that in the total sample of 176 participants, the cue exposure condition consumed significantly less kcal of exposed foods $(M=-0.33, S D=0.74)$ than the control condition $(M=0.40, S D=1.11), t(132.44)=5.01, p<.001, d=0.77$. However, the exposure effect did not generalise to consumption of non-exposed foods, as intake in the cue exposure condition $(M=-0.10, S D=1.03)$ was not significantly different from intake in the control condition $(M=0.12, S D=0.93), t(174)=1.43, p=.155, d=0.22$. Correlations between $\mathrm{kcal}$ intake and habituation of eating desires and (violation of) overeating expectancies are presented in Table 1 (including the data of chapter 3 ) and Table 2 (excluding the data of chapter 3).

As can be seen in Tables 1 and 2, neither WSH nor BSH of eating desires correlated significantly with intake during the taste test. In contrast, post-intervention overeating expectancies correlated significantly positively with intake of exposed foods (Tables 1 and 2 ), indicating that higher overeating expectancies are related to more intake. In addition, expectancy violation was found to be significantly negatively associated with intake of exposed (Tables 1 and 2) and non-exposed foods (Table 2 only). The negative correlations indicate that stronger violation of expectancies are significantly associated with less intake, indicating that stronger reductions of expectancies during cue exposure indeed were

3 CS-US expectancy data in chapter 3 were transformed as follows: $1=0 ; 2=25 ; 3=50 ; 4=75 ; 5=100$. 
beneficial. Of note, post-intervention expectancies were significantly negatively correlated with WSH, both when including data of all studies, $r(132)=-.31, p<.001$, and when excluding data from chapter $3, r(100)=-.45, p<.001$. Expectancy violation was also found to be significantly positively correlated with WSH, both when including, $r(132)=.20, p$ $=.019$, and excluding data of chapter $3, r(100)=.41, p<.001$, indicating that lower postintervention expectancies and a stronger reduction of expectancies during cue exposure are associated with a stronger habituation of eating desires during exposure sessions. The associations $\mathrm{BSH}$ and post-intervention overeating expectancies were not significant, $r(86)=-.14, p=.186$ (including chapter 3 ), or, $r(54)=-.23, \mathrm{p}=.089$ (excluding chapter 3 ), neither were the associations between BSH and expectancy violation, $\mathrm{r}(86)=.11, \mathrm{p}=.317$ (including chapter 3 ), or, $\mathrm{r}(54)=.25, \mathrm{p}=.074$ (excluding chapter 3 ).

Overall, it can be concluded that across the four studies in this dissertation, cue exposure significantly reduces overeating of foods included in exposure sessions, but it does not reduce the intake of non-exposed foods. Stronger habituation of eating desires either within or between exposure sessions is not associated with less overeating. In contrast, lower overeating expectancies after cue exposure and stronger reductions of such expectancies during cue exposure are associated with less overeating after cue exposure, although effects are small to medium. The weak correlations between within-session habituation and overeating expectancies suggest that these concepts only partly overlap.

Table 1. Correlations between $z$ scores of kcal intake and habituation of eating desires, post-intervention overeating expectancies and expectancy violation (pre minus post expectancies) among participants (children and adults) of all chapters (2, 3, 5 and 6$)$

\begin{tabular}{|c|c|c|c|c|}
\hline & $\begin{array}{c}\text { WSH of eating } \\
\text { desires } \\
n=140\end{array}$ & $\begin{array}{c}\text { BSH of eating } \\
\text { desires } \\
n=94\end{array}$ & $\begin{array}{c}\text { Post-intervention } \\
\text { expectancies } \\
n=168\end{array}$ & $\begin{array}{c}\text { Expectancy } \\
\text { violation } \\
n=150\end{array}$ \\
\hline$z$ intake exposed food & -.05 & -.08 & $.32^{* *}$ & $-.18^{*}$ \\
\hline$z$ intake non-exposed food & .02 & -.06 & .07 & -.12 \\
\hline
\end{tabular}

${ }^{*} p<.05 ;{ }^{*} p<.01 ; \mathrm{WSH}=$ within-session habituation; $\mathrm{BSH}=$ between-session habituation; Please note that sample sizes of each correlation vary due to measurement differences between studies.

Table 2. Correlations between $z$ scores of kcal intake and habituation of eating desires, post-intervention overeating expectancies and expectancy violation (pre minus post expectancies) among adult participants of chapters 2,5 and 6

\begin{tabular}{|lcccc|}
$\begin{array}{c}\text { WSH of eating } \\
\text { desires } \\
n=100\end{array}$ & $\begin{array}{c}\text { BSH of eating } \\
\text { desires } \\
n=54\end{array}$ & $\begin{array}{c}\text { Post-intervention } \\
\text { expectancies } \\
n=136\end{array}$ & $\begin{array}{c}\text { Expectancy } \\
\text { violation } \\
n=118\end{array}$ \\
\hline$z$ intake exposed food & -.09 & -.02 & $.41^{*}$ & $-.23^{*}$ \\
$z$ intake non-exposed food & -.05 & .03 & .12 & $-.19^{*}$ \\
\hline
\end{tabular}

${ }^{*} p<.05 ;{ }^{*} \mathrm{p}<.01$; WSH = within-session habituation; BSH = between-session habituation; Please note that sample sizes of each correlation vary due to measurement differences between studies. 

CHAPTER

GENERAL DISCUSSION 

Three main issues are central in this dissertation: (1a) To investigate whether food cue exposure reduces the overeating of foods that were included during exposure therapy (i.e., exposed foods), and (1b) whether these intake effects generalise to non-exposed foods, as described in chapters 2, 3, 5, 6 and 7, (2) to examine whether effects of food cue exposure on treatment outcomes can be explained by habituation of cue reactivity, and/or by expectancy violation, as described in chapters 2, 3, 5, 6 and 7, and lastly (3), to investigate whether a food cue exposure therapy decreases overeating, binge eating and overweight, as described in chapters $4-6$. Below, the main findings regarding each question will be summarized and discussed, and the methodological shortcomings and considerations for future research will be discussed. Finally, some recommendations will be provided for food cue exposure in clinical practice, as well as the conclusions regarding the three central issues.

\section{SUMMARY OF THE FINDINGS}

\section{(1) DOES FOOD CUE EXPOSURE REDUCE THE OVEREATING OF FOODS USED IN CUE EXPOSURE EXERCISES (I.E., EXPOSED FOODS; 1A), AND DO THESE INTAKE EFFECTS GENERALISE TO NON-EXPOSED FOODS (1B)?}

The studies in chapters 2, 3, 5 and 6 were all designed to answer this first central question. In all studies, participants in a food cue exposure condition received one or several cue exposure sessions with individually tailored foods. Prior to the intervention, participants indicated which foods were their most favourites, and most difficult to refrain from. In studies 2, 3 and 6, four foods were selected for food cue exposure, while all relevant foods were selected for the food cue exposure treatment in chapter 5. During the food cue exposure sessions, participants were instructed to hold, smell, touch and lick the foods that were presented on a tray, without actually eating the foods. To answer the central question, in each experiment, kcal intake of both exposed and non-exposed foods during a bogus taste test after the intervention was compared between a food cue exposure condition and a control condition. For the distinction of exposed and non-exposed foods in the studies in chapters 2 and 3, one standard food item was additionally added to the cue exposure tray for every participant in the cue exposure condition: chocolate mousse. During the bogus taste test in these chapters, six desserts were presented to the participant, of which only chocolate mousse had been included during food cue exposure. In both studies, it was found that participants in the cue exposure condition consumed significantly less kcal from chocolate mousse, the exposed food item, compared to participants in the control condition. Generalisation of the exposure effect to non-exposed foods was not found in chapter 2, but was found in chapter 3 . In the chapter 3 study, participants also ate less kcal 
from the non-exposed items in the cue exposure versus control condition.

While standard foods were included in the taste test in chapters 2 and 3, individually tailored foods were used for the taste test in chapters 5 and 6 . In chapter 5 , participants received 8 sessions of food cue exposure and an average of 28 homework exposures, in which all personal overeating foods were included, except for one food item that was carefully excluded from the cue exposure exercises (during the sessions and homework) for the purpose of the taste test. In this study, one of the personal exposed foods, as well as the personal non-exposed food were included in the bogus taste test. In addition, participants also selected one general food item from a list of 10 foods, which was also included in the cue exposure sessions, and included in the taste test. Results showed that directly after treatment, food cue exposure therapy, compared to a control treatment aimed at improving lifestyle, led to significantly less intake of the personal exposed food, but not to less intake of the personal non-exposed food or the general exposed food. At three months follow-up, intake differences between conditions of the personal exposed food were still marginally significant, whereas the differences between conditions for the personal nonexposed food and general exposed food remained non-significant. Finally, in chapter 6, four personally tailored foods were selected by the participants, of which two foods were randomly assigned as exposed foods and included in two food cue exposure sessions. All four foods were included in the bogus test and, again, results showed that food cue exposure led to significantly less kcal intake of exposed foods compared to a no-treatment control condition, but no significant differences were found between the food cue exposure and control condition for non-exposed foods.

When aggregating the intake data from all studies in chapters 2, 3, 5 and 6, it was found that food cue exposure leads to significantly less intake of exposed foods (1a), but cue exposure effects did not generalise to less intake of non-exposed foods (1b).

\section{(2) CAN THE EFFECTS OF FOOD CUE EXPOSURE ON TREATMENT OUTCOMES BE EXPLAINED BY HABITUATION OF CUE REACTIVITY, AND/OR BY EXPECTANCY VIOLATION?}

Given the findings in the anxiety exposure research that stronger habituation of fear responses during exposure sessions is not associated with better treatment outcome (Craske et al., 2008), the necessity of habituation of cue reactivity for outcomes of food cue exposure was investigated. In order to assess habituation of cue reactivity, eating desires were assessed at regular time-points during food cue exposure (chapters 2, 3, 5 and 6). In addition to eating desires, measurements of salivation were also included as a measure of cue reactivity (chapters 2 and 3). Within-session habituation (WSH) was measured by subtracting the end cue reactivity per session from the session's peak, and averaged over 
sessions when a participant received more than one exposure session. In addition, in case of several cue exposure sessions, the cue reactivity peak from the final session was subtracted from the peak of the first session as a measure of between-session habituation (BSH). When aggregating the data of WSH and BSH of eating desires and intake during the bogus taste test of all studies (chapter 7), no significant correlations were found between habituation of desires and intake. Associations between habituation of salivary responses and intake were also not found (chapters 2 and 3). Further, in the study described in chapter 5, binge eating reduction and weight loss were included as treatment outcomes. Results showed that habituation of eating desires was not related to binge eating reduction, but it was significantly related to weight loss at post-treatment. The latter association was found in an unexpected direction, as stronger WSH of eating desires was related to less weight loss. At three months follow-up, no significant associations were found for WSH or BSH of eating desires and weight loss or binge eating reduction.

Inhibitory learning has been suggested to be central to extinction (Bouton, 1993; Bouton \& King, 1983). In anxiety disorders, expectancy violation has been suggested as a strategy to enhance inhibitory learning of the CS-noUS association, which can be achieved by introducing CSs (e.g., high heart-rate, sweating) that maximize the mismatch between the expectancy of the US to take place (e.g., heart attack) and the actual outcome (e.g., the absence of the heart attack; noUS) (Craske, Treanor, Conway, Zbozinek, \& Vervliet, 2014). Since CS-US expectancies may also play a significant role in overeating, such as 'If I feel exhausted and chocolate is available (CS), then I will lose control and eat the entire chocolate bar (US)', it was investigated whether expectancy violation is a working mechanism of food cue exposure. To answer this question, CS-US expectancies were measured before and after food cue exposure, and the control interventions. Across the four studies in this dissertation (chapter 7), a significant positive correlation was found between the strength of post-intervention CS-US expectancies and kcal intake of exposed foods, indicating that ending food cue exposure with higher expectancies is related to more overeating. In addition, stronger violation (i.e., reduction) of CS-US expectancies was significantly related to less intake of exposed and non-exposed foods. In contrast to the significant relation between expectancy violation and intake, no associations were found between expectancy violation and weight loss or binge eating reduction (chapter 5).

In chapter 6 , it was investigated whether explicitly targeting expectancy violation during food cue exposure was more effective than targeting habituation of eating desires in a sample of obese females. Both exposure conditions received two individual sessions of food cue exposure, after which intake of exposed and non-exposed foods was measured. It was found that both exposure conditions consumed significantly less kcal from the exposed foods compared to the no-treatment control condition, while no differences in intake were found between both exposure conditions. No differences were found between all three 
conditions regarding the consumption of non-exposed foods. In addition, the length of exposure was not significantly different between conditions, suggesting that focussing food cue exposure on expectancy violation was not more efficient than focussing on habituation. Habituation and expectancy violation were measured during the food cue exposure sessions in both conditions: while habituation was not significantly different between both exposure conditions, expectancy violation during sessions was stronger in the expectancy violation condition than in the habituation condition. However, while expectancies during exposure sessions diminished stronger in the expectancy violation condition, the reduction of CSUS expectancies after finishing exposure sessions was comparably strong in both exposure conditions.

Summarizing, results of the studies in the present dissertation suggest that reduced posttherapy CS-US expectancies and stronger expectancy violation during food cue exposure are associated with reduced intake, while habituation of cue reactivity is generally found not to be associated with intake. However, results also suggest that explicitly targeting expectancy violation during food cue exposure does not lead to less overeating after cue exposure compared to targeting habituation of eating desires.

\section{(3) CAN FOOD CUE EXPOSURE THERAPY BE HELPFUL TO DECREASE OVEREATING, BINGE EATING, AND CAN IT BE USED TO ACHIEVE WEIGHT LOSS?}

In chapter 4, the design of a food cue exposure therapy study is described in which several techniques to strengthen inhibitory learning in food cue exposure therapy are used (Craske et al., 2014; Jansen, 1998; Jansen, Broekmate, \& Heymans, 1992). Although many of these techniques are used in clinical practice, the effects of using these techniques have not yet been investigated. The main approach for food cue exposure sessions was to target expectancy violation instead of habituation of eating desires. Before therapy, participants engaged in an extensive interview during which individual favourite foods that are generally involved in overeating and/or binge eating (USs) and food cues (CSs) were unravelled. Also, specific CS-US expectancies were analysed. Both were documented in a report that was delivered to the therapist, and used to design exposure exercises during sessions and for homework. Deepened extinction during exposure therapy was done by including several food cues after conducting cue exposure with at least one of the cues (such as adding specific television series, time and mood (CSs) after cue exposure to only the sight and smell (CSs)). The cue exposure sessions were done in multiple contexts, ensuring sufficient variability of lengths of exposure sessions, as well as hunger levels, and included occasional reinforcements during exposure exercises (i.e., taking small bites of food during exposure exercises). Further, safety signals were removed as much as possible (i.e., therapists leaving the room during exposures, and including homework exercises), and mental retrieval cues 
were included in the protocol (i.e., mental rehearsal of learning experiences during therapy). In chapter 5, the results of the cue exposure study are presented. Compared to participants in the control condition who received an extensive lifestyle intervention, it was found that participants in the cue exposure condition overate significantly less after the intervention, as previously described under the first central question. In addition, participants in the cue exposure condition lost significantly more weight at post-treatment and at follow-up, and binge eating frequency was marginally more reduced in the cue exposure versus control condition at post-measurement, and significantly more reduced at follow-up.

\section{GENERAL DISCUSSION}

\section{FOOD CUE EXPOSURE EFFECTS ON OVEREATING}

In all four studies described in this dissertation, it was consistently found that food cue exposure led to significantly less kcal intake of the foods that were included in exposure therapy, also in the absence of hunger. This effect was found shortly after the exposure in all studies, and a marginally significant intake reduction was still present at the three months follow-up after eight sessions of food cue exposure therapy. We designed one of the studies in a way that allowed us to directly compare the consumption of foods that were tailored to the individual (i.e., foods rated as highly palatable and difficult to refrain from), with consumption of the most palatable food item of a general list of 10 snack foods. While both the individual and general food items were included in exposure therapy, with a similar number of exposures per participant ( 7 to 8 exposures), significant differences between the exposure and control condition on kcal consumption were found for the personally selected food item only, while no differences between conditions were found for the general palatable food item (chapter 5). Thus, food cue exposure successfully leads to less overeating of foods that were included in cue exposure exercises. The data additionally show that the intake reducing effect of food cue exposure mainly involves foods that the participants considers as 'problematic': the effect of food cue exposure was found for the personally tailored food involved in overeating and not for the general palatable food. While two of our studies did show reduced intake after exposure with general foods, the study that compared personal versus general foods suggest that the use of personal foods will lead to better results. This finding is of special interest for clinical practice: it seems to be the most effective to include personalised food items that are associated with a loss of control in food cue exposure.

Though we found a strong exposure effect on intake, we failed to find generalisation of the intake effect. Less consumption of non-exposed foods was not found after one or two sessions of food cue exposure, and also not after eight sessions of food cue exposure therapy 
with an average of 28 homework exercises. There was however one exception; generalisation was found after two cue exposure sessions in the study with children and adolescents (chapter 3). Finding generalisation of cue exposure in youth might be a consequence of children being less burdened with an ingrained learning history, so they might be more malleable than adults and might be better able to change existing behavioural patterns. Alternatively, finding generalisation of exposure in this study could have been a coincidental finding, and therefore needs to be replicated in future studies. When looking at the data of all studies combined (chapter 7), it can be concluded that food cue exposure does not lead to less overeating of non-exposed foods. Finding no generalisation in the majority of studies fits well with findings in other research areas. For example, Van den Hout (2011) and colleagues found that after two exposure sessions for contamination fears, participants reported less feelings of contamination, danger, fear, and disgust of the feared object that was included during exposure therapy, but these effects did not generalise to non-exposed objects. Likewise, exposure to a certain feared movement in patients with chronic pain, led to less overprediction of pain concerning that particular movement, but not to less overprediction of pain in non-exposed movements (Crombez et al., 2002; Goubert, Francken, Crombez, Vansteenwegen, \& Lysens, 2002).

Finding no generalisation to non-exposed food items, feared objects or movements might be less surprising when it is considered that extinction is stimulus-specific (Vervliet, Vansteenwegen, Baeyens, Hermans, \& Eelen, 2005; Vervliet, Vansteenwegen, \& Eelen, 2004). Vervliet and colleagues $(2005,2004)$ found that extinction with a stimulus that was somewhat different from the originally conditioned stimulus (i.e., the generalisation stimulus) led to increased fear responding at test - when the original conditioned stimulus was presented again, compared to a control condition in which participants received extinction with the originally conditioned stimulus. The authors concluded that return of fear after exposure therapy can be elicited by phobic stimuli that are perceptually different from the stimuli used during exposure; extinction is stimulus-specific. Translating these findings to food cue exposure: it might be better to use personally tailored food items that have been involved in an individual's learning history of overeating and/or binge eating (analogue to the conditioned stimulus) instead of general food items (analogue to the generalisation stimulus) during food cue exposure to achieve reductions of overeating or binge eating of personally relevant foods.

However, results by Vervliet and colleagues do indicate that generalisation of extinction effects is possible (Vervliet et al., 2005, 2004). In their conditioning experiments, results showed that extinction with a conditioned stimulus leads to less responding to stimuli that share features with the extinguished stimulus (i.e., generalisation stimuli). The authors predicted that it would be helpful to include originally conditioned stimuli during exposure therapy, as this would decrease fear when being confronted with variations of the phobic stimulus in the future (Vervliet et al., 2004). This finding of less responding to generalisation 
stimuli was found for stimuli with perceptual similarities with the conditioned stimulus (i.e., the shape of the generalisation stimulus looked a bit different from the shape of the conditioned stimulus; Vervliet et al., 2005, 2004), but also for stimuli that share conceptual similarities (i.e., the generalisation stimulus belonging to the same conceptual category as the conditioned stimulus; Vervoort, Vervliet, Bennett, \& Baeyens, 2014). These findings might explain why generalisation to other tasty foods was not found in chapters 4 and 6: in both these studies, non-exposed personalised food items were included during the taste test to measure generalisation, while, as mentioned before, personalised foods were selected based on their learning history with overeating, and might therefore be considered conditioned stimuli. The true test of generalisation of the food cue exposure effect would therefore be to not include personalised non-exposed food items in the taste test, but food items that are variations of the personalised exposed food that have not been (extensively) involved in overeating or binge eating.

When translating the findings of Vervliet and colleagues $(2005,2004)$ to food cue exposure, it might be worthwhile to include perceptual variants of exposed foods in taste tests. For example, if Tony Chocolonely milk chocolate is involved in overeating or binge eating occasions for a specific person, other shapes of chocolate (e.g., thicker/ thinner bars, larger/ smaller pieces, white/ dark chocolate) and other brands of chocolate (e.g., Milka/ Cote D'Or/ Verkade) could be included in the taste test as generalisation foods. Also other food products belonging to the 'chocolate class' might be included, such as chocolate cake, chocolate spread, chocolate ice cream or chocolate mousse. In addition to perceptual variations of exposed foods, conceptual variations could also be considered. For example, chocolate could be considered belonging to the category sweet high-caloric snacks, and (non-chocolate-containing) cookies, candy or cupcakes could therefore also be included in the taste test as generalisation foods. Based on the work in the anxiety field on generalisation gradients (e.g., Armony, Servan-schreiber, Lizabeth, Cohen, \& Ledoux, 1997), it would be predicted that the larger the perceived similarities between the tested generalising food and exposed food, the less kcal would be consumed of the generalisation food. This idea provides an alternative explanation for the (only) significant generalisation finding in chapter 3, as only in chapter 2 and 3, non-personal foods (and therefore not conditioned stimuli) were included in the taste tests, whereas personally tailored foods (and therefore conditioned stimuli) were used in the taste tests in chapters 4 and 6. In the taste test in chapter 2 and 3, six desserts were included of which chocolate mousse was the standard exposed food item. When taking a closer look at the other non-exposed food items in the taste test, there is one food item that might be regarded as a close variant of the exposed chocolate mousse: chocolate cake. To test this post hoc hypothesis that food cue exposure does lead to reduced intake of variations of exposed foods, additional analyses were conducted on the data in chapter 3 . These analyses suggest that the generalisation 
effect of intake of non-exposed foods was partly driven by less intake of the chocolate cake. The effect size of the difference between conditions was smaller for chocolate cake intake, $t(27.32)=2.02, p=.053, d=0.65$, than for chocolate mousse intake, $t(25.32)=2.52, p=$ $.018, d=0.80$. In addition, the generalisation effect of all non-exposed food items became marginally significant when chocolate cake was excluded from this analysis, $t(26.69)=1.86$, $p=.074, d=0.60$, compared to a significant difference when chocolate cake was included, $t(28.58)=2.32, p=.028, d=0.74$. Speculatively, the fact that intake of non-exposed foods was still (marginally) less in the cue exposure condition without chocolate cake might be explained by the idea that the other non-exposed food items (i.e., muffin, whipped cream, strawberry mousse and custard) in the taste test could arguably also be seen as (conceptual) variations of chocolate mousse, as all items were sweet high-caloric desserts, though all more distinctive that chocolate cake.

The finding that food cue exposure therapy led to significant weight loss in the cue exposure condition in chapter 5 might also support the hypothesis that food cue exposure effects do generalise to non-exposed foods. It might be assumed that weight loss was not merely the result of eating less from the few specific problematic foods that were included in therapy, but also eating less from a broader range of high-caloric foods that are variations of the personally exposed foods. Thus, food cue exposure to personally selected food items might have led to extinction of other related food items in daily life as well and accumulatively led to weight loss. Future research could systematically study this issue of generalisation of food cue exposure effects to perceptual and conceptual variations of exposed foods.

\section{HABITUATION VERSUS EXPECTANCY VIOLATION AS WORKING MECHANISMS OF FOOD CUE EXPOSURE}

Anxiety research shows that stronger habituation during exposure sessions is not consistently related to better treatment outcomes (Craske et al., 2008), while all published food cue exposure studies use habituation as a criterion to determine the end of sessions and therapy. Across the four food cue exposure studies described in this dissertation, habituation of cue reactivity within (WSH) and between (BSH) cue exposure sessions was generally found not to be associated with the studies' outcome measures. Habituation of salivary responses was not found to be associated with food intake (chapters 2 and 3), and also habituation of eating desires was generally not found to be associated with intake, weight loss and binge eating reduction (chapters 2, 3, 5, 6 and 7).

As an alternative for habituation as a working mechanism for exposure therapy, anxiety exposure research suggests that exposure therapy works through violation of US expectancies (Craske et al., 2008, 2014). While exposure sessions for anxiety disorders aim at maximizing the expectancy of the catastrophic event to occur by including all 
important conditioned stimuli during exposure, food cue exposure sessions might aim at maximizing the expectancy of losing control over eating behaviour. The exposure session should result in a significant reduction of CS-US expectancies, with the prediction that stronger reduction of expectancies are associated with better treatment outcome (Craske et al., 2014). Across the four studies in this dissertation (chapter 7), it was indeed found that lower post-therapy CS-US (i.e., overeating) expectancies were related to less intake of the exposed foods. Moreover, stronger violation (i.e., reduction) of CS-US expectancies during exposure therapy was correlated with less intake of exposed and non-exposed foods. While the violation of CS-US expectancies during the food cue exposure therapy was found to be a significant and full mediator for kcal intake of exposed foods in chapter 5, this was not found for weight loss and binge eating reduction.

As the abovementioned findings on the relationship between expectancy violation and intake were based on correlations, it was investigated in chapter 6 whether explicitly targeting expectancy violation during food cue exposure was more effective than targeting habituation of eating desires. The results of this study showed that targeting expectancy violation during food cue exposure led to a significantly stronger violation of CS-US expectancies during exposure sessions compared to food cue exposure targeting habituation of eating desires, while habituation of eating desires was observed in both conditions. While the no-treatment control condition ate significantly more kcal of exposed foods compared to both food cue exposure conditions, the expectancy violation condition was not superior to the habituation condition. Moreover, the length of exposure was not significantly different between conditions, suggesting that focussing food cue exposure on expectancy violation was not more efficient than focussing on habituation. A remarkable finding was that, even though expectancies during exposure sessions diminished stronger in the expectancy violation condition, the reduction of CS-US expectancies after finishing exposure sessions was comparably strong in both exposure conditions. This suggests that even without explicitly targeting CS-US expectancies during exposure sessions, doing cue exposure exercises eventually leads to a violation of expectancies. After all, all food cue exposure participants were successful in keeping control of their food desires during the exposure exercises, as none of the participants actually lost control during cue exposure. It is possible that similar CS-US expectancy reductions are caused by the assessment of expectancies, which might have primed participants and might have influenced learning.

Alternatively, it could be reasoned that an explicit focus on the violation of expectancies during exposure is not necessary for expectancies to change. Only a few anxiety exposure studies found that explicitly aiming exposure sessions at challenging expectancies or beliefs leads to superior outcomes than purely behavioural exposure sessions. Salkovskis and colleagues (2006) found a clear benefit of focusing on violation of expectancies during exposure therapy compared to exposure therapy aimed at habituation of fear in a pilot study 
of 16 panic disorder patients with agoraphobia. In this study, participants in the expectancy violation condition reported less panic attacks, anxiety and avoidance of fearful situations after therapy, and also dared to encounter more fearful situations in a behavioural test, compared to the habituation condition in which exposure exercises to feared situations were followed in a hierarchical order. Evidence for the importance of targeting expectancies during exposure was also found in another study in students suffering from anxiety specific for respiratory sensations (Deacon et al., 2013). In this study, an intensive exposure condition, in which participants received as much 60-second exposure trials as needed (with a minimum of eight trials) to reduce the feared outcome, was compared to 'standard' exposure conditions, in which a standard number of three 60-second exposure trials were used, and progress to the next exposure trial was based on sufficient habituation of body sensations. Participants in the intensive exposure condition experienced less fear of bodily sensations after the intervention, and also reported stronger reduction of expectancies of the feared outcome to occur during the intervention, compared to the standard exposure conditions. This stronger reduction of expectancies also turned out to be a significant mediator for better treatment outcomes. Important to point out in this study is the fact that the superior intensive exposure condition received much more exposure compared to the standard exposure conditions, while the content of the exposure sessions was the same for all conditions. Thus, while the results by Deacon and colleagues (2013) indeed indicate that expectancies are important for treatment outcome, it does not provide strong evidence for the hypothesis that explicitly directing exposure sessions at expectancy violation is absolutely necessary for optimal treatment effects.

In fact, Koch, Spates and Himle (2004) found evidence that challenges the need of explicitly tackling expectancies in exposure therapy for small animal phobias. In that study, patients who completed a purely behavioural treatment profited as much from the treatment as patients who received a cognitive-behavioural treatment, in which dysfunctional cognitions were challenged by behavioural tests. In addition to the similar effects in both conditions on behavioural and phobic symptoms, both conditions experienced an equal change in cognitions and needed an equal duration of exposure (i.e., the number of minutes required to achieve the behavioural target of contact with the animal) to achieve these effects (Koch et al., 2004). The same was found in an experiment in spider phobics, in which explicitly targeting maladaptive cognition did not have added benefit as compared to a purely behavioural treatment (Raes, Koster, Loeys, \& De Raedt, 2011). Interestingly, the change in maladaptive cognitions in both conditions was again found to mediate the improvement in self-related and spider-related fears (Raes et al., 2011). These latter findings are generally in line with the findings in this dissertation: the food cue exposure condition aimed at expectancy violation in chapter 6 did not lead to less intake than the food cue exposure condition aimed at habituation of eating desires, while reduction of expectancies 
after therapy was equally strong in both exposure conditions. In addition, findings of chapter 7 show that the reduction of overeating expectancies was related to reduced kcal intake.

In addition to relatively few exposure studies on the importance of explicitly tackling expectancy violation, no fear conditioning studies have been conducted to experimentally disentangle the two extinction approaches. One appetitive conditioning study has recently been conducted to test the importance of expectancy violation for extinction of conditioned chocolate desires (van den Akker, van den Broek, Havermans, \& Jansen, 2016). After healthy-weight participants acquired chocolate expectancies in an acquisition phase, a regular extinction procedure was compared to an extinction procedure during which eating expectancies were explicitly disconfirmed (i.e., instructed extinction). The results indicated that explicit belief disconfirmation did not lead to less eating desires compared to the regular extinction condition, neither during the actual extinction procedure nor during a second extinction procedure one day later (i.e., re-extinction), showing that targeting expectancy violation does not lead to better extinction (van den Akker et al., 2016).

A further notable finding in chapter 6 was the correlation between the reduction of CS-US expectancies and the reduction of eating desires during the cue exposure sessions. This association was also observed in chapter 7: stronger habituation of eating desires was associated with lower post-intervention expectancies and stronger reduction of expectancies during therapy. This finding seems especially puzzling since overeating expectancies were found to be significantly correlated with intake, but habituation of eating desires was not. However, the weak correlations between habituation and expectancy violation of the accumulated data of all four cue exposure studies in chapter 7 suggests that both concepts only partly overlap, and thus reflect, at least partly, different cognitive processes.

Summarizing, lower CS-US expectancies after therapy as well as stronger violation of expectancies during therapy are significantly associated with less overeating, while stronger habituation of cue reactivity during food cue exposure sessions is not associated with better treatment outcome. In addition, results suggest that explicitly targeting expectancy violation during food cue exposure is not necessary for better treatment outcome, as expectancies also change during exposure sessions without explicitly tackling them.

\section{FOOD CUE EXPOSURE THERAPY FOR OVEREATING, BINGE EATING REDUCTION AND WEIGHT LOSS}

In contrast to the short cue exposure interventions in chapters 2, 3 and 6, a more extensive food cue exposure intervention was tested in chapter 5 to address the question: can food cue exposure therapy successfully lead to less overeating, binge eating and, ultimately, weight loss? As described in chapter 4, the eight-session cue exposure intervention included many techniques to maximize inhibitory learning and thus treatment effects. Therapy comprised of two-weekly individual sessions in personal overeating contexts for 
one month, during which the therapist attempted to tackle all CS-US associations that were revealed during a pre-therapy interview, complemented by an average of 28 homework exercises. As mentioned previously, compared to an active Lifestyle + control intervention, participants in the cue exposure condition overate significantly less directly after therapy, and marginally less at follow-up. In addition, participants receiving food cue exposure therapy lost more weight directly after therapy, as well as at three months follow-up. While previous studies have reported weight loss in a more comprehensive program containing some cue exposure elements, and prevention of weight regain after weight loss (Boutelle, Knatz, Carlson, Bergmann, \& Peterson, 2016; Mount, Neziroglu, \& Taylor, 1990), the study in chapter 5 presents weight loss results of a pure cue exposure intervention. Although effect sizes were medium to large (2.1\% weight loss in food cue exposure condition vs. $0.4 \%$ weight loss in control condition), and weight loss was maintained at three months followup, the weight loss was still limited in terms of clinical relevance, and the long-term effects of food cue exposure therapy are unknown. After all, the greatest challenge is not to initially lose weight, but to keep the weight off (Wing \& Phelan, 2005).

An important risk factor for weight cycling and weight regain is the presence of binge eating (Mcguire, Wing, Klem, Lang, \& Hill, 1999; Venditti, Wing, Jakicic, Butler, \& Marcus, 1996). As much as $30 \%$ of the (adolescent) participants in chapter 3 and $41 \%$ of the (adult) participants in chapter 5 reported binge eating, which is in line with percentages found in obese adolescent and adult samples of previous studies (e.g., Berkowitz, Stunkard, \& Stallings, 1993; Decaluwé, Braet, \& Fairburn, 2003; Marcus, Wing, \& Lamparski, 1985; Spitzer et al., 1993). While the presence of binge eating did not change after the 2 -session protocol in chapter 3, binge eating frequency was successfully reduced in the extensive cue exposure condition in chapter 5. Directly after therapy, the number of binges was reduced to zero in the cue exposure condition. At follow-up, the reduction of binge eating relative to pre-measurement was still significantly larger in the cue exposure condition compared to the control condition, although it should be noted that this could be due to the marginally higher frequency at pre-measurement. Pure cue exposure therapy has previously been found to be very beneficial for binge eating in bulimia nervosa patients (Jansen et al., 1992; Jansen, Hout, van den, Loof, de, Zandbergen, \& Griez, 1989; Martinez-mallen et al., 2007; Toro et al., 2003). 


\section{METHODOLOGICAL CONSIDERATIONS}

The described studies in this dissertation have advanced the field of food cue exposure therapy for overeaters by testing cue exposure in well-controlled research designs on the specificity of cue exposure effects, and investigating the presumed working mechanisms of food cue exposure. A notable strength in this dissertation is the fact that the effect of food cue exposure on reduced intake of exposed foods was replicated repeatedly in our own studies, suggesting a robust effect that is not the result of type I error. In addition, control conditions were included in every experiment, in contrast to most food cue exposure studies published so far (Boutelle et al., 2016; Jansen, Van den Hout, De Loof, Zandbergen, \& Griez, 1989; Martinez-mallen et al., 2007; Rosen \& Leitenberg, 1982; Schmidt \& Marks, 1988; Toro et al., 2003). A further point worth mentioning is that food cue exposure was investigated in participants with weight and eating problems, not in analogue (student) samples. However, some limitations should also be noted. In all studies, the sample sizes were limited, which poses a general problem for the reliability of study findings and the risk of type II errors, though an effort was made to overcome this power problem by aggregating all data in chapter 7 . In light the of general need for replication of research findings, it would be highly valuable to replicate the study described in chapter 6 with a larger sample size, to state any definite conclusions about the necessity of explicitly targeting expectancy violation during food cue exposure. Further, for the adult samples in chapters 2, 5 and 6, only females were included, limiting the generalisability of the findings in this dissertation to adult females. Finally, effects of food cue exposure on weight loss and binge eating were not studied longer than three months after therapy; studying long term exposure effects on weight cycling after one to several years is warranted for the utility for clinical practice.

\section{FUTURE RESEARCH}

As research on cue exposure's effects and mechanisms is a relatively understudied research domain, while it appears to be a very effective intervention to reduce overeating, many exciting research questions await further investigation, of which a few are worth mentioning.

1) It is possible that generally not finding generalisation in this dissertation is due to the sub-optimal generalisation test. An important and exciting direction for future research would therefore be to systematically test generalisation to perceptual and conceptual variations of the exposed foods after food cue exposure.

2) Further, it would be interesting to separately study the effects of the techniques to enhance inhibitory learning that have all been combined in the design of chapter 4 , and altogether were responsible for the beneficial effects on weight loss and binge eating 
in chapter 5. For example, to study the effects of food cue exposure with and without occasional reinforcements.

3) In addition, it would be highly interesting to investigate whether certain subgroups within the obese population might profit more from food cue exposure than others. A few subgroups might deserve extra attention in the future:

a. Children. It might be highly valuable to investigate whether food cue exposure therapy works better for children and adolescents than for adults. Given the results of chapter 3 , it would be of special interest to compare generalisation of exposure effects to non-exposed foods in children versus adults.

b. Binge eaters. Another subgroup of interest are the participants who suffer from binge eating episodes, who might have especially distinctive CS-US associations that, once tackled during cue exposure, might result in better treatment outcomes compared to overweight or obese participants who 'graze' all day therefore might have more diffuse CS-US associations.

c. Individuals with weak inhibition skills. Recently, it has been suggested by Jansen and colleagues (2016) that inhibitory learning might be related to the ability to inhibit one's responses to cues, suggesting that exposure might be less effective in individuals with weak inhibition skills. Indeed, it has been shown that participants with weak inhibition skills showed slower extinction of appetitive responses in a conditioning study, compared to participants with stronger inhibition skills (van den Akker, Havermans, Bouton, \& Jansen, 2014), although this was not found in other studies (e.g., Papachristou, Nederkoorn, Beunen, \& Jansen, 2013; van den Akker, Havermans, \& Jansen, 2015). The importance of inhibition skills seems especially worthwhile to study in overweight and obese individuals, as several studies found associations between overeating/ obesity and weak inhibitory skills (e.g., Guerrieri, Nederkoorn, \& Jansen, 2012; Nederkoorn, Houben, Hofmann, Roefs, \& Jansen, 2010; Nederkoorn, Smulders, Havermans, Roefs, \& Jansen, 2006), also in children and adolescents (e.g., Batterink, Yokum, \& Stice, 2010; Nederkoorn, Braet, Van Eijs, Tanghe, \& Jansen, 2006). It would be worthwhile examining whether participants with especially bad inhibition skills would show slower inhibitory learning during food cue exposure compared to participants with strong inhibition skills. And, if so, how these skills can be optimally improved. 


\section{RECOMMENDATIONS FOR CLINICAL PRACTICE}

Based on the studies in this dissertation, it can be concluded that food cue exposure can be a valuable intervention to incorporate in weight loss interventions for obesity. A few recommendations can be formulated for the application of cue exposure in clinical practice, which are partly based on the studies in the present dissertation, but also based on the translation of findings of a broader range of fundamental extinction and exposure studies.

First, therapists should pay careful attention to choosing food items that are experienced as highly palatable and have been associated with problems in inhibitory control by the individual patient; i.e., food items with a learning history of overeating and/or binge eating. These food items need to be brand and type-specific, and not random high-caloric snack foods. Doing exposure exercises with a specific problematic food item is helpful to reduce overeating of that specific food item. However, while future research is necessary to study generalisation of cue exposure effects to variations of exposed problematic food items, it seems that exposure effects do not easily generalise to non-exposed specific problematic food items. Therefore, as a second recommendation, therapists should carefully make an inventory of all specific problematic food items, and they need to make sure that all these foods are sufficiently addressed during exposure sessions and homework exercises. Although this certainly requires some planning for the therapists and the patients to bring relevant foods to the sessions, most gains can likely be made through homework exposure that need to be well-prepared during therapy sessions. Third, in addition to exposing patients to all 'proximal' food cues, such as seeing, holding, smelling and tasting the food, therapist should also carefully investigate all important CS-US associations, such as external cues (e.g., time of day, the absence of family members), internal cues (e.g., feelings of exhaustion, feeling hungry), and contexts (e.g., own living room, gas station), and include these cues in the exposures accordingly. For example, when the feeling of extreme hunger is an important CS for an eating binge, hunger should be arranged during the exposure, for example by including a period of food deprivation before the cue exposure session. Other relevant internal cues can be mood states, which may be more difficult to incorporate during therapy sessions, but can excellently be prepared through homework exercises. Finally, although habituation of eating desires is still a widely used target for cue exposure sessions, no convincing evidence was found for the necessity to focus on eating desires during cue exposure sessions, as stronger habituation was generally found to be unrelated to better treatment outcomes. Instead, results of the present dissertation suggest that it is important that overeating expectancies reduce during food cue exposure. More research is necessary to investigate whether explicitly targeting CS-US expectancies during food cue exposure is essential to reduce these expectancies, as research so far suggests that expectancies can reduce even when focussing food cue exposure on eating desires. For now, therapists could aim at overeating expectancies, eating desires, or both. 


\section{CONCLUSION}

Answering to the three central questions in this dissertation, it is concluded that:

(1a) Food cue exposure reduces the eating of food items used during the exposure, and (1b) food cue exposure effects do not generalise to non-exposed foods. It is unclear whether and to what extent cue exposure effects do generalise to variations of the exposed foods, and to which variations precisely, due to a lack of proper generalisation tests.

(2) Stronger violation of expectancies is associated with reduced intake, while habituation of eating desires is generally not found to be associated with less intake. Future replication research is warranted to investigate whether explicitly tackling expectancy violation during food cue exposure is necessary.

(3) Food cue exposure therapy successfully leads to less overeating, binge eating, and leads to weight loss. Studying the long-term effects of food cue exposure therapy remains an important goal for the future. 



American Psychiatric Association (2013). Diagnostic and statistical manual of mental disorders (5th edition). Arlington, VA: American Psychiatric Publishing.

Armony, J. L., Servan-schreiber, D., Lizabeth, M., Cohen, J. D., \& Ledoux, J. E. (1997). Stimulus Generalization of Fear Responses : Effects of Auditory Cortex Lesions in a Computational Model and in Rats. Cerebral Cortex, 7(2), 157-165.

Baker, A., Mystkowski, J., Culver, N., Yi, R., Mortazavi, A., \& Craske, M. G. (2010). Does habituation matter? Emotional processing theory and exposure therapy for acrophobia. Behaviour Research and Therapy, 48(11), 1139-1143. https://doi.org/10.1016/j. brat.2010.07.009

Baron, K. G., Reid, K. J., Kern, A. S., \& Zee, P. C. (2011). Role of sleep timing in caloric intake and BMI. Obesity (Silver Spring, Md.), 19(7), 1374-1381. https://doi.org/10.1038/ oby.2011.100

Batterink, L., Yokum, S., \& Stice, E. (2010). Body mass correlates inversely with inhibitory control in response to food among adolescent girls: An fMRI study. NeuroImage, 52(4), 1696-1703. https://doi.org/10.1016/j.neuroimage.2010.05.059

Berkowitz, R., Stunkard, A. J., \& Stallings, V. A. (1993). Binge-eating disorder in obese adolescent girls. Annals of the New York Academy of Sciences, 699, 200-206. https://doi. org/10.1111/j.1749-6632.1993.tb18850.x

Birch, L. L., \& Fisher, J. O. (2000). Mothers' child-feeding practices influence daughters' eating and weight. The American Journal of Clinical Nutrition, 71(5), 1054-1061.

Bish, C. L., Blanck, H. M., Serdula, M. K., Marcus, M., Kohl, H. W., \& Khan, L. K. (2005). Diet and physical activity behaviors among Americans trying to lose weight: 2000 Behavioral Risk Factor Surveillance System. Obesity Research, 13(3), 596-607. https:// doi.org/10.1038/oby.2005.64

Boggiano, M., Dorsey, J., Thomas, J., \& Murdaugh, D. (2009). The Pavlovian power of palatable food: lessons for weight-loss adherence from a new rodent model of cueinduced overeating. International Journal of Obesity, 33(6), 693-701.

Bongers, P., \& Jansen, A. (2017). Emotional eating and Pavlovian learning: evidence for conditioned appetitive responding to negative emotional states. Cognition and Emotion, 31(2), 284-297. https://doi.org/10.1080/02699931.2015.1108903

Bongers, P., van den Akker, K., Havermans, R., \& Jansen, A. (2015). Emotional eating and Pavlovian learning: Does negative mood facilitate appetitive conditioning? Appetite, 89, 226-236. https://doi.org/10.1016/j.appet.2015.02.018

Bordens, K. S., \& Abbott, B. B. (2011). Research designs and methods. A process approach (8th ed.). New York, NY: McGraw-Hill. 
Boswell, R. G., \& Kober, H. (2016). Food cue reactivity and craving predict eating and weight gain: A meta-analytic review. Obesity Reviews, 17, 159-177. https://doi.org/10.1111/ obr.12354

Boutelle, K. N., Knatz, S., Carlson, J., Bergmann, K., \& Peterson, C. B. (2017). An Open Trial Targeting Food Cue Reactivity and Satiety Sensitivity in Overweight and Obese Binge Eaters. Cognitive and Behavioral Practice, 24, 363-373. https://doi.org/http:// dx.doi.org/10.1016/j.cbpra.2016.08.003

Boutelle, K. N., Zucker, N. L., Peterson, C. B., Rydell, S. A., Cafri, G., \& Harnack, L. (2011). Two novel treatments to reduce overeating in overweight children: a randomized controlled trial. Journal of Consulting and Clinical Psychology, 79(6), 759-771. https:// doi.org/10.1037/a0025713

Boutelle, K. N., Zucker, N., Peterson, C. B., Rydell, S., Carlson, J., \& Harnack, L. J. (2014). An intervention based on Schachter's externality theory for overweight children: the regulation of cues pilot. Journal of Pediatric Psychology, 39(4), 405-417. https://doi. org/10.1093/jpepsy/jst142

Bouton, M. E. (1988). Context and ambiguity in the extinction of emotional learning: implications for exposure therapy. Behaviour Research and Therapy, 26(2), 137-149. Retrieved from http://www.ncbi.nlm.nih.gov/pubmed/3365204

Bouton, M. E. (1993). Context, Time, and Memory Retrieval in the Interference Paradigms of Pavlovian Learning. Psychological Bulletin, 114(1), 80-99. https://doi.org/10.1037/00332909.114.1.80

Bouton, M. E. (2011). Learning and the persistence of appetite: extinction and the motivation to eat and overeat. Physiology \& Behavior, 103(1), 51-58. https://doi.org/10.1016/j. physbeh.2010.11.025

Bouton, M. E., \& King, D. A. (1983). Contextual control of the extinction of conditioned fear: tests for the associative value of the context. Journal of Experimental Psychology: Animal Behavior Processes, 9(3), 248-265. https://doi.org/10.1037/0097-7403.9.3.248

Bouton, M. E., Woods, A. M., \& Pineño, O. (2004). Occasional reinforced trials during extinction can slow the rate of rapid reacquisition. Learning and Motivation, 35(4), 371390. https://doi.org/10.1016/j.lmot.2004.05.001

Bulik, C. M., Sullivan, P. F., Carter, F. C., McIntosh, V., \& Joyce, P. (1998). The role of exposure with response prevention in the cognitive-behavioral therapy for bulimia nervosa. Psychological Medicine, 28, 611-623. 
Butte, N. F., Cai, G., Cole, S. A., Wilson, T. A., Fisher, J. O., Zakeri, I. F., ... Comuzzie, A. G. (2007). Metabolic and behavioral predictors of weight gain in Hispanic children: The Viva la Familia Study. American Journal of Clinical Nutrition, 85(6), 1478-1485. https:// doi.org/85/6/1478 [pii]

Buysse, D. J., Reynolds, C. F. 3rd, Monk, T. H., Berman, S. R., \& Kupfer, D. J. (1989). The Pittsburgh Sleep Quality Index: A New Instrument for Psychiatric Practice and Research. Psychiatry Research, 28, 193-213. https://doi.org/10.1016/0165-1781(89)90047-4

Cole, T. J., Bellizzi, M. C., Flegal, K. M., \& Dietz, W. H. (2000). Establishing a standard definition for child overweight and obesity worldwide: international survey. BMJ, 320, 1-6.

Craske, M. G., Kircanski, K., Zelikowsky, M., Mystkowski, J., Chowdhury, N., \& Baker, A. (2008). Optimizing inhibitory learning during exposure therapy. Behaviour Research and Therapy, 46(1), 5-27. https://doi.org/10.1016/j.brat.2007.10.003

Craske, M. G., Liao, B., Brown, L., \& Vervliet, B. (2012). The role of inhibition in exposure therapy. Journal of Experimental Psychopathology, 3(3), 322-345. doi: 10.5127/jep.026511

Craske, M. G., Treanor, M., Conway, C. C., Zbozinek, T., \& Vervliet, B. (2014). Maximizing exposure therapy: An inhibitory learning approach. Behaviour Research and Therapy, 58, 10-23. https://doi.org/10.1016/j.brat.2014.04.006

Crombez, G., Eccleston, C., Vlaeyen, J. W. S., Vansteenwegen, D., Lysens, R., \& Eelen, P. (2002). Exposure to physical movements in low back pain patients: restricted effects of generalization. Health Psychology, 21(6), 573-578. https://doi.org/10.1037//02786133.21.6.573

Cuddy, A. J. C., Wilmuth, C. A., Yap, A. J., \& Carney, D. R. (2015). Preparatory Power Posing Affects Nonverbal Presence and Job Interview Performance. Journal of Applied Psychology, 100(4), 1286-1295. http://dx.doi.org/10.1037/a0038543

Culver, N. C., Stoyanova, M., \& Craske, M. G. (2011). Clinical relevance of retrieval cues for attenuating context renewal of fear. Journal of Anxiety Disorders, 25(2), 284-292. https://doi.org/10.1016/j.janxdis.2010.10.002

Curioni, C. C., \& Lourenco, P. M. (2005). Long-term weight loss after diet and exercise: a systematic review. International Journal of Obesity, 29(29), 1168-1174. https://doi.org/ doi:10.1038/sj.ijo.0803015

Deacon, B. J., \& Abramowitz, J. S. (2004). Cognitive and behavioral treatments for anxiety disorders: a review of meta-analytic findings. Journal of Clinical Psychology, 60(4), 429441. https://doi.org/10.1002/jclp.10255 
Deacon, B. J., Kemp, J. J., Dixon, L. J., Sy, J. T., Farrell, N. R., \& Zhang, A. R. (2013). Maximizing the efficacy of interoceptive exposure by optimizing inhibitory learning: a randomized controlled trial. Behaviour Research and Therapy, 51(9), 588-596. https:// doi.org/10.1016/j.brat.2013.06.006

Decaluwé, V., Braet, C., \& Fairburn, C. G. (2003). Binge eating in obese children and adolescents. International Journal of Eating Disorders, 33(1), 78-84. https://doi. org/10.1002/eat.10110

Dibbets, P., Havermans, R., \& Arntz, A. (2008). All we need is a cue to remember: the effect of an extinction cue on renewal. Behaviour Research and Therapy, 46(9), 1070-1077. https://doi.org/10.1016/j.brat.2008.05.007

Dibbets, P., Moor, C., \& Voncken, M. J. (2013). The effect of a retrieval cue on the return of spider fear. Journal of Behavior Therapy and Experimental Psychiatry, 44(4), 361-367. https://doi.org/10.1016/j.jbtep.2013.03.005

Duckworth, A. L., \& Kern, M. L. (2011). A meta-analysis of the convergent validity of self-control measures. Journal of Research in Personality, 45(3), 259-268. https://doi. org/10.1016/j.jrp.2011.02.004

Elsesser, K., Wannemüller, A., Lohrmann, T., Jöhren, P., \& Sartory, G. (2013). Mental retrieval of treatment context in dental phobia. Behavioural and Cognitive Psychotherapy, 41(2), 173-187. https://doi.org/10.1017/S1352465812000318

Fairburn, C. G., \& Beglin, S. J. (1994). Assessment of eating disorders: interview or selfreport questionnaire? The International Journal of Eating Disorders, 16(4), 363-370. https://doi.org/10.1002/1098-108X(199412)

FAO/WHO/UNU. (2001). Human energy requirements: Report of a Joint FAO/WHO/ UNU Expert Consultation. FAO Food and Nutrition Technical Report Series. https://doi. org/9251052123

Ferriday, D., \& Brunstrom, J. M. (2011). “I just can't help myself': effects of food-cue exposure in overweight and lean individuals. International Journal of Obesity, 35(1), 142-149. https://doi.org/10.1038/ijo.2010.117

Finer, N. (2015). Medical consequences of obesity. Medicine (United Kingdom), 43(2), 8893. https://doi.org/10.1016/j.mpmed.2014.11.003

Flegal, K. M., Kruszon-Moran, D., Carroll, M. D., Fryar, C. D., \& Ogden, C. L. (2016). Trends in obesity among adults in the united states, 2005 to 2014. JAMA, 315(21), 22842291. https://doi.org/10.1001/jama.2016.6458

Foa, E. B., \& Kozak, M. J. (1986). Emotional processing of fear: exposure to corrective information. Psychological Bulletin, 99(1), 20-35. Retrieved from http://www.ncbi.nlm. nih.gov/pubmed/2871574 
Foa, E. B., \& McNally, R. J. (1996). Mechanisms of change in exposure therapy. In M. Rapee (Ed.), Current controversies in the anxiety disorders (pp. 329-343). New York: The Guilford Press.

Franz, M. J., VanWormer, J. J., Crain, A. L., Boucher, J. L., Histon, T., Caplan, W., ... Pronk, N. P. (2007). Weight-Loss Outcomes: A Systematic Review and Meta-Analysis of Weight-Loss Clinical Trials with a Minimum 1-Year Follow-Up. Journal of the American Dietetic Association, 107(10), 1755-1767. https://doi.org/10.1016/j.jada.2007.07.017

Goubert, L., Francken, G., Crombez, G., Vansteenwegen, D., \& Lysens, R. (2002). Exposure to physical movement in chronic back pain patients: No evidence for generalization across different movements. Behaviour Research and Therapy, 40(4), 415-429. https:// doi.org/10.1016/S0005-7967(01)00020-1

Gruijters, S. L. K. (2016). Baseline comparisons and covariate fishing: Bad statistical habits we should have broken yesterday. European Health Psychologist, 18(5), 205-209.

Guerrieri, R., Nederkoorn, C., \& Jansen, A. (2012). Disinhibition is easier learned than inhibition. The effects of (dis)inhibition training on food intake. Appetite, 59(1), 96-99. https://doi.org/10.1016/j.appet.2012.04.006

Holm, S. (1979). A Simple Sequentially Rejective Multiple Test Procedure. Scandinavian Journal of Statistics, 6(2), 65-70.

Hou, R., Mogg, K., Bradley, B. P., Moss-Morris, R., Peveler, R., \& Roefs, A. (2011). External eating, impulsivity and attentional bias to food cues. Appetite, 56(2), 424-427. https:// doi.org/10.1016/j.appet.2011.01.019

Houben, K., Nederkoorn, C., \& Jansen, A. (2014). Eating on impulse: The relation between overweight and food-specific inhibitory control. Obesity, 22(5), 2013-2015. https://doi. org/10.1002/oby.20670

Jansen, A. (1998). A learning model of binge eating: cue reactivity and cue exposure. Behaviour Research and Therapy, 36(3), 257-272. Retrieved from http://www.ncbi.nlm. nih.gov/pubmed/9642846

Jansen, A., Broekmate, J., \& Heymans, M. (1992). Cue-exposure vs self-control in the treatment of binge eating: a pilot study. Behaviour Research and Therapy, 30(3), 235241. Retrieved from http://www.ncbi.nlm.nih.gov/pubmed/1586360

Jansen, A., Havermans, R., \& Nederkoorn, C. (2011(a)). Cued Overeating. In V. R. Preedy, R. R. Watson, \& C. R. Martin (Eds.), The International Handbook of Behavior, Diet and Nutrition (pp. 1431-1443). New York: Springer.

Jansen, A., Houben, K., \& Roefs, A. (2015). A cognitive profile of obesity and its translation into new interventions. Frontiers in Psychology, 6, 1-9. https://doi.org/10.3389/ fpsyg.2015.01807 
Jansen, A., Nederkoorn, C., Roefs, A., Bongers, P., Teugels, T., \& Havermans, R. (2011(b)). The proof of the pudding is in the eating: is the DEBQ-external eating scale a valid measure of external eating? The International Journal of Eating Disorders, 44(2), 164-8. https://doi.org/10.1002/eat.20799

Jansen, A., Schyns, G., Bongers, P., \& van den Akker, K. (2016). From lab to clinic: Extinction of cued cravings to reduce overeating. Physiology and Behavior, 162, 174-180. https:// doi.org/10.1016/j.physbeh.2016.03.018

Jansen, A., Stegerman, S., Roefs, A., Nederkoorn, C., \& Havermans, R. (2010). Decreased salivation to food cues in formerly obese successful dieters. Psychotherapy and Psychosomatics, 79(4), 257-258. https://doi.org/10.1159/000315131

Jansen, A., Theunissen, N., Slechten, K., Nederkoorn, C., Boon, B., Mulkens, S., \& Roefs, A. (2003). Overweight children overeat after exposure to food cues. Eating Behaviors, 4(2), 197-209. https://doi.org/10.1016/S1471-0153(03)00011-4

Jansen, A., Van den Hout, M. A., De Loof, C., Zandbergen, J., \& Griez, E. (1989). A case of bulimia Successfully treated by cue exposure. Journal of Behavior Therapy and Experimental Psychiatry, 20(4), 327-332.

Jansen, A., Voorwinde, V., Hoebink, Y., Rekkers, M., Martijn, C., \& Mulkens, S. (2016). Mirror exposure to increase body satisfaction: Should we guide the focus of attention towards positively or negatively evaluated body parts? Journal of Behavior Therapy and Experimental Psychiatry, 50, 90-96.

Jansen, E., Mulkens, S., Hamers, H., \& Jansen, A. (2007). Assessing eating disordered behaviour in overweight children and adolescents: bridging the gap between a selfreport questionnaire and a gold standard interview. Netherlands Journal of Psychology, 63(3), 93-97. https://doi.org/10.1007/BF03061070

Kendall, P. C., Robin, J. A., Hedtke, K. A., Suveg, C., Flannery-Schroeder, E., \& Gosch, E. (2005).

Considering CBT with anxious youth? Think exposures. Cognitive and Behavioral Practice, 12(1), 136-148. https://doi.org/10.1016/S1077-7229(05)80048-3

Kleim, B., Wilhelm, F., Temp, L., Margraf, J., Wiederhold, B., \& Rasch, B. (2014). Sleep enhances exposure therapy. Psychological Medicine, 44(7), 1511-1519. doi:10.1017/ S0033291713001748

Koch, E. I., Spates, C. R., \& Himle, J. A. (2004). Comparison of behavioral and cognitivebehavioral one-session exposure treatments for small animal phobias. Behaviour Research and Therapy, 42(12), 1483-1504. https://doi.org/10.1016/j.brat.2003.10.005 
Kral, T. V., Allison, D. B., Birch, L. L., Stallings, V. A., Moore, R. H., \& Faith, M. S. (2013). Caloric compensation and eating in the absence of hunger in 5- to 12 -y-old weightdiscordant siblings. The Amercian Journal of Clinical Nutrition, 96, 574-583. https://doi. org/10.3945/ajcn.112.037952

Logan, G. D., Schachar, R. J., \& Tannock, R. (1997). Impulsivity and Inhibitory Control. Psychological Science, 8(1), 60-64.

Lowe, M. R., \& Butryn, M. L. (2007). Hedonic hunger: a new dimension of appetite? Physiology \& Behavior, 91(4), 432-439. https://doi.org/10.1016/j.physbeh.2007.04.006

Maloney, P. W., Grawitch, M. J., \& Barber, L. K. (2012). The multi-factor structure of the Brief Self-Control Scale: Discriminant validity of restraint and impulsivity. Journal of Research in Personality, 46(1), 111-115. https://doi.org/10.1016/j.jrp.2011.10.001

Marcus, M. D., Wing, R. R., \& Lamparski, D. M. (1985). Binge eating and dietary restraint in obese patients. Addictive Behaviors, 10(2), 163-168. https://doi.org/10.1016/03064603(85)90022-X

Martinez-Mallén, E., Castro-Fornieles, J., La, L., Moreno, E., Morer, A., Font, E., \& Julien, J. (2007). Cue Exposure in the Treatment of Resistant Adolescent Bulimia Nervosa. International Journal of Eating Disorders, 40, 596-601. https://doi.org/10.1002/eat

Mcguire, M. T., Wing, R. R., Klem, M. L., Lang, W., \& Hill, J. O. (1999). What predicts weight regain in a group of successful weight losers? Journal of Consulting and Clinical Psychology, 67(2), 177-185. https://doi.org/10.1037/0022-006X.67.3.282

McIntosh, V. V. W., Carter, F. A., Bulik, C. M., Frampton, C. M. A., \& Joyce, P. R. (2011). Fiveyear outcome of cognitive behavioral therapy and exposure with response prevention for bulimia nervosa. Psychological Medicine, 41(5), 1061-1071. https://doi.org/10.1017/ S0033291710001583

Mount, R., Neziroglu, F., \& Taylor, C. J. (1990). An obsessive-compulsive view of obesity and its treatment. Journal of Clinical Psychology, 46(1), 68-78.

Mustelin, L., Bulik, C. M., Kaprio, J., \& Keski-Rahkonen, A. (2017). Prevalence and correlates of binge eating disorder related features in the community. Appetite, 109, 165-171. https://doi.org/10.1016/j.appet.2016.11.032

Mystkowski, J. L., Craske, M. G., Echiverri, A. M., \& Labus, J. S. (2006). Mental reinstatement of context and return of fear in spider-fearful participants. Behavior Therapy, 37(1), 4960. https://doi.org/10.1016/j.beth.2005.04.001

Nederkoorn, C., \& Jansen, A. (2002). Cue reactivity and regulation of food intake. Eating Behaviors, 3, 61-72. 
Nederkoorn, C., Braet, C., Van Eijs, Y., Tanghe, A., \& Jansen, A. (2006). Why obese children cannot resist food: the role of impulsivity. Eating Behaviors, 7(4), 315-322. https://doi. org/10.1016/j.eatbeh.2005.11.005

Nederkoorn, C., Houben, K., Hofmann, W., Roefs, A., \& Jansen, A. (2010). Control yourself or just eat what you like? Weight gain over a year is predicted by an interactive effect of response inhibition and implicit preference for snack foods. Health Psychology, 29(4), 389-393. https://doi.org/10.1037/a0019921

Nederkoorn, C., Jansen, E., Mulkens, S., \& Jansen, A. (2007). Impulsivity predicts treatment outcome in obese children. Behaviour Research and Therapy, 45(5), 1071-1075. https:// doi.org/10.1016/j.brat.2006.05.009

Nederkoorn, C., Smulders, F. T. Y., Havermans, R. C., Roefs, A., \& Jansen, A. (2006). Impulsivity in obese women. Appetite, 47(2), 253-256. https://doi.org/10.1016/j. appet.2006.05.008

Nederkoorn, C., Smulders, F. T., \& Jansen, A. (2000). Cephalic phase responses, craving and food intake in normal subjects. Appetite, 35(1), 45-55. https://doi.org/10.1006/ appe. 2000.0328

Ng, M., Fleming, T., Robinson, M., Thomson, B., Graetz, N., Margono, C., ... Gakidou, E. (2014). Global, regional, and national prevalence of overweight and obesity in children and adults during 1980-2013: A systematic analysis for the Global Burden of Disease Study 2013. The Lancet, 384(9945), 766-781. https://doi.org/10.1016/S01406736(14)60460-8

Ogden, C. L., Carroll, M. D., Kit, B. K., \& Flegal, K. M. (2014). Prevalence of childhood and adult obesity in the United States, 2011-2012. JAMA, 311(8), 806-814. doi: 10.1001/ jama.2014.732

Owen, R., \& Spencer, R. M. C. (2013). Body ideals in women after viewing images of typical and healthy weight models. Body Image, 10(4), 489-494. https://doi.org/10.1016/j. bodyim.2013.04.005

Pace-Schott, E. F., Verga, P. W., Bennett, T. S., \& Spencer, R. M. C. (2012). Sleep promotes consolidation and generalization of extinction learning in simulated exposure therapy for spider fear. Journal of Psychiatric Research, 46(8), 1036-1044. https://doi. org/10.1016/j.jpsychires.2012.04.015

Papachristou, H., Nederkoorn, C., Beunen, S., \& Jansen, A. (2013). Dissection of appetitive conditioning. Does impulsivity play a role? Appetite, 69, 46-53. https://doi.org/10.1016/j. appet.2013.05.011

Pavlov, I. P. (1927). Conditioned reflexes. London: Oxford University Press. 
Peters, J., Kalivas, P. W., \& Quirk, G. J. (2009). Extinction circuits for fear and addiction overlap in prefrontal cortex. Learning \& Memory, 16(5), 279-288. https://doi. org/10.1101/lm.1041309

Preacher, K. J., \& Hayes, A. F. (2004). SPSS and SAS procedures for estimating indirect effects in simple mediation models. Behavior Research Methods, Instruments, \& Computers, 36(4), 717-731. https://doi.org/10.3758/BF03206553

Rachman, S., Craske, M., Tallman, K., \& Solyom, C. (1986). Does escape behavior strengthen agoraphobic avoidance? A replication. Behavior Therapy, 17(4), 366-384. https://doi. org/http://dx.doi.org/10.1016/S0005-7894(86)80069-7

Rachman, S., Robinson, S., \& Lopatka, C. (1987). Is incomplete fear-reduction followed by a return of fear? Behaviour Research and Therapy, 25(1), 67-69. doi: http://dx.doi. org/10.1016/0005-7967(87)90116-1

Raes, A. K., Koster, E. H. W., Loeys, T., \& De Raedt, R. (2011). Pathways to change in one-sessionexposure with and without cognitive intervention: an exploratory study in spider phobia. Journal of Anxiety Disorders, 25(7), 964-971. https://doi.org/10.1016/j. janxdis.2011.06.003

Rijkeboer, M. M., \& Van den Hout, M. A. (2014). Nieuwe inzichten over exposure. Gedragstherapie, 47(1), 2-19.

Roefs, A., Houben, K., \& Werthmann, J. (2015). Desire for Food and the Power of Mind. In W. Hofmann \& L. F. Nordgren (Eds.), The Psychology of Desire (pp. 323-346). New York: Guilford Press. https://doi.org/10.1017/CBO9781107415324.004

Rosen, J. C., \& Leitenberg, H. (1982). Bulimia Nervosa: Treatment with Exposure and Response Prevention. Behavior Therapy, 13, 117-124.

Salkovskis, P. M., Hackmann, A., Wells, A., Gelder, M. G., \& Clark, D. M. (2006). Belief disconfirmation versus habituation approaches to situational exposure in panic disorder with agoraphobia: a pilot study. Behaviour Research and Therapy, 45(5), 877-885. https:// doi.org/10.1016/j.brat.2006.02.008

Schmidt, U., \& Marks, I. (1988). Cue exposure to food plus response prevention of binges for bulimia: A pilot study. International Journal of Eating Disorders, 7(5), 663-672.

Schyns, G., Roefs, A., Mulkens, S., \& Jansen, A. (2016). Expectancy violation, reduction of food cue reactivity and less eating in the absence of hunger after one food cue exposure session for overweight and obese women. Behaviour Research and Therapy, 76, 57-64. https://doi.org/10.1016/j.brat.2015.11.007 
Schyns, G., Roefs, A., Smulders, F. T. Y., \& Jansen, A. (2018). Cue exposure therapy reduces overeating of exposed and non-exposed foods in obese adolescents. Journal of Behavior Therapy and Experimental Psychiatry, 58, 68-77. https://doi.org/10.1016/j. jbtep.2017.09.002

Serdula, M. K., Ivery, D., Coates, R. J., Freedman, D. S., Williamson, D. F., \& Byers, T. (1993). Do obese children become obese adults? A review of the literature. Preventive Medicine, 22, 167-177. https://doi.org/10.1006/pmed.1993.1014

Shah, P. B. (2011). Intention-to-treat and per-protocol analysis. CMAJ, 183(6), 695. https:// doi.org/10.1503/cmaj.111-2031

Shiban, Y., Pauli, P., \& Mühlberger, A. (2013). Effect of multiple context exposure on renewal in spider phobia. Behaviour Research and Therapy, 51(2), 68-74. https://doi. org/10.1016/j.brat.2012.10.007

Sonneville, K. R. (2013). Longitudinal Associations Between Binge Eating and Overeating and Adverse Outcomes Among Adolescents and Young Adults. Does Loss of Control Matter? Archives of Pediatrics \& Adolescent Medicine, 2, 149-155. https://doi. org/10.1001/2013.jamapediatrics.12

Spitzer, R. L., Yanovski, S., Wadden, T., Wing, R., Marcus, M. D., Stunkard, A., ... Horne, R. L. (1993). Binge eating disorder: its further validation in a multisite study. The International Journal of Eating Disorders, 13(2), 137-153. https://doi.org/10.1002/1098108X(199303)13:2<137::AID-EAT2260130202>3.0.CO;2-\#

Spoormaker, V. I., Sturm, A., Andrade, K., Schröter, M., Goya-Maldonado, R., Holsboer, F., ... Czisch, M. (2010). The neural correlates and temporal sequence of the relationship between shock exposure, disturbed sleep and impaired consolidation of fear extinction. Journal of psychiatric research, 44(16), 1121-1128. doi: 10.1016/j.jpsychires.2010.04.017

Spoormaker, V. I., Schröter, M. S., Andrade, K. C., Dresler, M., Kiem, S. a, Goya-Maldonado, R., ... Czisch, M. (2012). Effects of rapid eye movement sleep deprivation on fear extinction recall and prediction error signaling. Human Brain Mapping, 33(10), 23622376. https://doi.org/10.1002/hbm.21369

Tangney, J. P., Baumeister, R. F., \& Boone, A. L. (2004). High self-control predicts good adjustment, less pathology, better grades, and interpersonal success. Journal of Personality, 72(2), 271-324. https://doi.org/10.1111/j.0022-3506.2004.00263.x

Tanofsky-Kraff, M., Yanovski, S. Z., Schvey, N. A, Olsen, C. H., Gustafson, J., \& Yanovski, J. A. (2009). A prospective study of loss of control eating for body weight gain in children at high risk for adult obesity. The International Journal of Eating Disorders, 42(1), 26-30. https://doi.org/10.1002/eat.20580 
Telch, C., Agras, W. S., \& Rossiter, E. M. (1988). Binge eating increases with increasing adiposity. International Journal of Eating Disorders, 7(1), 115-119. https://doi. org/10.1002/1098-108X(198801)7:1<115::AID-EAT2260070112>3.0.CO;2-W

The GBD 2015 Obesity Collaborators. (2017). Health Effects of Overweight and Obesity in 195 Countries over 25 Years. The New England Journal of Medicine, 377(1), 13-27. https://doi.org/10.1056/NEJMoa1614362

Toro, J., Cervera, M., Feliu, M. H., Garriga, N., Jou, M., Martinez, E., \& Toro, E. (2003). Cue exposure in the treatment of resistant bulimia nervosa. The International Journal of Eating Disorders, 34(2), 227-234. https://doi.org/10.1002/eat.10186

Twain, M. (1897). XLIX. In Following the equator (p. 459). Retrieved from http://www. goodreads.com/quotes/show/271379

Uzogara, S. G. (2017). Obesity Epidemic, Medical and Quality of Life Consequences: A Review. International Journal of Public Health Research, 5(1), 1-12. https://doi. org/7180284

Van den Akker, K., Bongers, P., Hanssen, I., \& Jansen, A. (2017). Validation of prospective portion size and latency to eat as measures of reactivity to snack foods. Appetite, 116, 480-486. https://doi.org/10.1016/j.appet.2017.05.049

Van den Akker, K., Havermans, R. C., Bouton, M. E., \& Jansen, A. (2014). How partial reinforcement of food cues affects the extinction and reacquisition of appetitive responses. A new model for dieting success? Appetite, 81, 242-252. https://doi. org/10.1016/j.appet.2014.06.024

Van den Akker, K., Havermans, R. C., \& Jansen, A. (2015). Effects of occasional reinforced trials during extinction on the reacquisition of conditioned responses to food cues. Journal of Behavior Therapy and Experimental Psychiatry, 48, 50-58. https://doi. org/10.1016/j.jbtep.2015.02.001

Van den Akker, K., Jansen, A., Frentz, F., \& Havermans, R. C. (2013). Impulsivity makes more susceptible to overeating after contextual appetitive conditioning. Appetite, 70, 73-80. https://doi.org/10.1016/j.appet.2013.06.092

Van den Akker, K., Schyns, G., \& Jansen, A. (2016). Enhancing inhibitory learning to reduce overeating: Design and rationale of a cue exposure therapy trial in overweight and obese women. Contemporary Clinical Trials, 49, 85-91. https://doi.org/10.1016/j. cct.2016.06.008

Van den Akker, K., van den Broek, M., Havermans, R. C., \& Jansen, A. (2016). Violation of eating expectancies does not reduce conditioned desires for chocolate. Appetite, 100, 10-17. https://doi.org/10.1016/j.appet.2016.02.004 
Van den Hout, M. A., Engelhard, I. M., Toffolo, M. B. J., \& van Uijen, S. L. (2011). Exposure plus response prevention versus exposure plus safety behaviours in reducing feelings of contamination, fear, danger and disgust. An extended replication of Rachman, Shafran, Radomsky \& Zysk (2011). Journal of Behavior Therapy and Experimental Psychiatry, 42(3), 364-70. https://doi.org/10.1016/j.jbtep.2011.02.009

Van Gucht, D., Vansteenwegen, D., Beckers, T., Hermans, D., Baeyens, F., \& Van den Bergh, O. (2008). Repeated cue exposure effects on subjective and physiological indices of chocolate craving. Appetite, 50, 19-24. doi: 10.1016/j.appet.2007.05.003

Van Gucht, D., Vansteenwegen, D., Van den Bergh, O., \& Beckers, T. (2008). Conditioned craving cues elicit an automatic approach tendency. Behaviour Research and Therapy, 46(10), 1160-1169. https://doi.org/10.1016/j.brat.2008.05.010

Vansteenwegen, D., Vervliet, B., Hermans, D., Beckers, T., Baeyens, F., \& Eelen, P. (2006). Stronger renewal in human fear conditioning when tested with an acquisition retrieval cue than with an extinction retrieval cue. Behaviour Research and Therapy, 44(12), 1717-1725. https://doi.org/10.1016/j.brat.2005.10.014

Venditti, E. M., Wing, R. R., Jakicic, J. M., Butler, B. A., \& Marcus, M. D. (1996). Weight cycling, psychological health, and binge eating in obese women. Journal of Consulting and Clinical Psychology, 64(2), 400-405. https://doi.org/10.1037/0022-006X.64.2.400

Vervliet, B., Vansteenwegen, D., Baeyens, F., Hermans, D., \& Eelen, P. (2005). Return of fear in a human differential conditioning paradigm caused by a return to the original acquistion context. Behaviour Research and Therapy, 43(3), 323-336. https://doi. org/10.1016/j.brat.2004.01.001

Vervliet, B., Vansteenwegen, D., \& Eelen, P. (2004). Generalization of extinguished skin conductance responding in human fear conditioning. Learning \& Memory, 11(5), 555558. https://doi.org/10.1101/lm.77404

Vervoort, E., Vervliet, B., Bennett, M., \& Baeyens, F. (2014). Generalization of human fear acquisition and extinction within a novel arbitrary stimulus category. PLoS ONE, 9(5). https://doi.org/10.1371/journal.pone.0096569

Voedingscentrum. (2011). Guidelines Food Choices. De Gezondheidsraad. Retrieved from www.voedingscentrum.nl

Vögele, C., \& Florin, I. (1997). Psychophysiological responses to food exposure: An experimental study in binge eaters. International Journal of Eating Disorders, 21(2), 147-157. https://doi.org/10.1002/(SICI)1098-108X(199703)21:2<147::AIDEAT5>3.0.CO;2-L 
Werthmann, J., Jansen, A., \& Roefs, A. (2015). Worry or craving? A selective review of evidence for food-related attention biases in obese individuals, eating-disorder patients, restrained eaters and healthy samples. The Proceedings of the Nutrition Society, 74(2), 99-114. https://doi.org/10.1017/S0029665114001451

Werthmann, J., Jansen, A., \& Roefs, A. (2016). Make up your mind about food: A healthy mindset attenuates attention for high-calorie food in restrained eaters. Appetite, 105, 53-59. https://doi.org/10.1016/j.appet.2016.05.005

Werthmann, J., Jansen, A., Vreugdenhil, A. C. E., Nederkoorn, C., Schyns, G., \& Roefs, A. (2015). Food Through the Child's Eye: An Eye-Tracking Study on Attentional Bias for Food in Healthy-Weight Children and Children With Obesity. Health Psychology, 34(12), 1123-1132. https://doi.org/10.1037/hea0000225

Werthmann, J., Roefs, A., Nederkoorn, C., Mogg, K., Bradley, B. P., \& Jansen, A. (2011). Can(not) take my eyes off it: Attention bias for food in overweight participants. Health Psychology, 30(5), 561-569. https://doi.org/10.1037/a0024291

World Health Organization (WHO). (2014). The challenge of obesity - quick statistics. 2014, Retrieved from http://www.euro.who.int/en/health-topics/noncommunicablediseases/obesity/data-and-statistics.

Wilson, G. T. (1994). Behavioral treatment of childhood obesity: Theoretical and practical implications. Health Psychology, 13(5), 371-372.

Wing, R. R., \& Phelan, S. (2005). Long-term weight loss maintenance. The American Journal of Clinical Nutrition, 82(1), 222-225. https://doi.org/82/1/222S [pii]

Zalta, A. K., Dowd, S., Rosenfield, D., Smits, J. a J., Otto, M. W., Simon, N. M., ... Pollack, M. H. (2013). Sleep quality predicts treatment outcome in CBT for social anxiety disorder. Depression and Anxiety, 30(11), 1114-1120. https://doi.org/10.1002/da.22170

Zhao, X., Lynch, J. G., \& Chen, Q. (2010). Reconsidering Baron and Kenny: Myths and Truths about Mediation Analysis. Journal of Consumer Research, 37(2), 197-206. https:// doi.org/10.1086/651257 


Strong food cue reactivity, such as heightened eating desires, is thought to play an important role in overeating and weight gain. Food cue reactivity is at least partly learned through classical conditioning, as food cues such as the smell and sight of food (conditioned stimuli or CSs) can become associated with eating (unconditioned stimulus or US) through repeated pairings, and these CSs can subsequently elicit cue reactivity. Because many eating desires are acquired through classical conditioning, extinction of such associations might be the appropriate way to decrease the learned cue reactivity. Exactly this is the aim of food cue exposure: to expose participants to CSs, such as the smell and sight of food, while food intake (US) is prevented. The few studies that have investigated the effects of food cue exposure therapy found that exposure leads to substantial reductions in overeating and binges eating, though cue exposure has mainly been studied in bulimia nervosa patients. Many important questions about the effects of cue exposure await investigation, and working mechanisms of food cue exposure have never been studied. Investigating the effects and working mechanisms of food cue exposure therapy for overweight and obese individuals is the goal of this dissertation.

Chapter 1 introduces food cue exposure therapy as a treatment for overeating and obesity. The role of classical conditioning in overeating is described, as well as the use of extinction principles in food cue exposure therapy. Prior research on the effects of food cue exposure therapy, and research on the potential working mechanisms of exposure therapy in the anxiety domain is outlined. Finally, the three main questions in this dissertation are raised: First, does food cue exposure reduce the overeating of foods used in cue exposure exercises (i.e., exposed foods), and do these intake effects generalise to non-exposed foods? Second, can the effects of food cue exposure on treatment outcomes be explained by habituation of cue reactivity, and/or by expectancy violation? And third, can food cue exposure therapy be helpful to decrease overeating, binge eating, and can it be used to achieve weight loss?

The studies described in chapters 2 and 3 are almost identical in experimental design: in both studies, overweight and obese participants (adult females in chapter 2, and 12-18-year-old boys and girls in chapter 3) were randomly assigned to a cue exposure intervention (one session in chapter 2 and two sessions in chapter 3), or a similar-length control intervention focusing on a healthy lifestyle and body image. Participants received the intervention in group-format, between 2 and 7 participants per group, though the behavioural dependent measure, eating in the absence of hunger, was measured individually after finishing the intervention. The selected foods were individually tailored: before the intervention, participants in the cue exposure conditions indicated online which food items were their most favourites, and most difficult to refrain from. Four favourite food items were selected for food cue exposure. One standard food item was added for every participant in the cue exposure condition: chocolate mousse. During the exposure 
exercises, participants were instructed to hold, smell, touch and lick the food, in order to get their eating desires as strong as possible. During both interventions, eating desires and salivation were regularly measured, in order to determine within and between-session habituation of cue reactivity, while CS-US expectancies were measured before and after the intervention, in order to determine the change of expectancies during therapy (i.e., expectancy violation). After the intervention, and in the absence of hunger, participants engaged in a bogus taste test. The bogus taste test included the exposed food item chocolate mousse, and five food items that were not used during food cue exposure. In both studies, it was found that participants in the cue exposure condition consumed significant less kcal from exposed food items compared to participants in the control condition. Generalization of the exposure effect to non-exposed foods was not found in chapter 2, but was found in chapter 3: in this study, participants also ate less kcal from the non-exposed items in the cue exposure versus control condition. In both studies, habituation of eating desires was not associated with kcal intake, while lower CS-US expectancies were related to less kcal intake in chapter 2, but not in chapter 3 .

Chapter 4 describes techniques to improve inhibitory learning in food cue exposure therapy, and the design of a study to investigate the effects of an eight-session food cue exposure therapy on eating desires, CS-US expectancies, eating behaviour and weight loss for obesity. In this study, the cue exposure intervention was compared to an extensive lifestyle intervention at post-treatment and at three months follow-up; the results of the study are described in chapter 5 . The main approach for exposure sessions was to target on expectancy violation instead of habituation of eating desires. Before therapy, participants engaged in an extensive interview during which individual favourite foods that are generally involved in overeating and/or binge eating (USs) and food cues (CSs) were unravelled. Also, specific CS-US expectancies were analysed. Both were documented in a report that was delivered to the therapist, and used to design exposure exercises during sessions and for homework. Deepened extinction during exposure therapy was done by including several food cues after conducting cue exposure with at least one of the cues (such as adding specific television series, time and mood (CSs) after cue exposure to only the sight and smell (CSs)). The cue exposure sessions were done in multiple overeating contexts, ensuring sufficient variability of lengths of exposure sessions, as well as hunger levels, including ocassional reinforcements during exposure exercises (i.e., taking small bites of food during exposure exercises), removal of safety signals as much as possible (i.e., therapists leaving the room during exposures, and including homework exercises), and including mental retrieval cues (i.e., mental rehearsal of learning experiences during therapy). Habituation of eating desires (in the cue exposure condition only) and expectancy violation (in both conditions) were determined in order to relate them to treatment effects. 
In chapter 5, the results of the study described in chapter 4 are presented. It was found that participants in the cue exposure condition lost significantly more weight at posttreatment and at follow-up than participants in the control condition. In addition, binge eating frequency was marginally more reduced in the cue exposure versus control condition at post-measurement, and significantly more reduced at follow-up. Regarding other overeating behaviour, participants in the cue exposure condition consumed significantly less from the exposed foods during the bogus taste test at post-measurement, and a trend was found for less consumption at follow-up. However, no differences were found for nonexposed and general snack foods. With regard to working mechanisms, the expectancy violation was significantly larger in the cue exposure condition compared to the control condition, and appeared to be a significant (full) mediator for kcal consumption of exposed foods. It did, however, not mediate weight loss and/or binge eating reduction. Habituation of eating desires within the exposure condition was not associated with better treatment outcome.

Lastly, in the experiment described in chapter 6, it was investigated whether explicitly targeting expectancy violation during food cue exposure was more effective than targeting habituation of eating desires. Obese females were randomized in a cue exposure condition aimed at expectancy violation, a cue exposure condition aimed at habituation of eating desires, or a no-treatment control condition. Both exposure conditions received two individual sessions of food cue exposure, after which consumption of exposed and nonexposed foods in the absence of hunger was measured. It was found that both exposure conditions consumed less kcal from the exposed foods compared to the control condition, while no differences in intake were found between both exposure conditions. No differences were found between all three conditions regarding the consumption of non-exposed foods. Moreover, the length of exposure was not significantly different between conditions, suggesting that focussing food cue exposure on expectancy violation was not more efficient than focussing on habituation. Habituation and expectancy violation were measured during exposure sessions, and expectancy violation was also measured from pre to posttherapy. Expectancy violation during sessions was stronger in the expectancy violation condition than in the habituation condition, while habituation was not significantly different between both exposure conditions. However, while expectancies during exposure sessions diminished stronger in the expectancy violation condition, the reduction of CSUS expectancies after finishing exposure sessions was comparably strong in both exposure conditions. Neither expectancy violation nor habituation of eating desires were found to be significantly associated with the intake of exposed foods, though lower expectancies at the end of the exposure significantly mediated the relationship between condition and less intake of the non-exposed, but not exposed, foods. 
In chapter 7, the data of all four studies (chapters 2, 3, 5 and 6) were aggregated into one dataset to increase power. Using this larger dataset, it was investigated whether cue exposure effects on intake of exposed foods do generalize to non-exposed foods. And in addition, the associations between habituation of cue reactivity, expectancy violation and $\mathrm{kcal}$ intake were investigated. It was found that, across the four studies in this dissertation, cue exposure significantly reduces overeating of foods included in exposure sessions, but it does not reduce the intake of non-exposed foods. Stronger habituation of eating desires, either within or between exposure sessions, was not found to be associated with less overeating. In contrast, lower overeating expectancies after cue exposure and stronger reductions of such expectancies during cue exposure were found to be associated with less overeating after cue exposure.

Finally, in chapter 8, the findings regarding main questions are summarized and discussed, and the methodological shortcomings and considerations for future research are discussed. Some recommendations are provided for food cue exposure in clinical practice, and conclusions are made regarding the three central issues in this dissertation. In short, it is concluded that food cue exposure reduces the overeating of food items used during the exposure, and that food cue exposure effects do not generalise to non-exposed foods (1). In addition, stronger violation of expectancies is associated with reduced intake, while habituation of eating desires is generally not found to be associated with less intake (2). And lastly, food cue exposure therapy leads to reductions of overeating, binge eating, and leads to weight loss (3). 


VALORISATION

ADDENDUM 

The present dissertation describes several studies investigating the effects and working mechanisms of cue exposure therapy for overweight and obese individuals. In this Valorisation Addendum, the societal and economic relevance of the findings of this dissertation are highlighted. In addition, an overview is provided of the target groups that might profit from the findings in this dissertation. Further, a number of recommendations have been formulated to improve cue exposure treatments in clinical practice. Also, activities in which these recommendations have been disseminated are mentioned, and finally, the innovation of the studies in this dissertation is highlighted.

\section{SOCIETAL RELEVANCE}

Worldwide, overweight and obesity prevalences increase disturbingly. Obesity has severe medical consequences, such as cardiovascular disease, type 2 diabetes, asthma and obstructive sleep apnoea. In addition, obesity has serious consequences for the quality of life, including weight discrimination, depression and mobility problems, altogether making obesity is one of the largest health issues of our time. In essence, obesity is the result of an imbalance between energy expenditure and energy input. However, the fact that weight loss interventions that aim purely at this negative energy balance (i.e., less energy intake than energy expenditure) rarely lead to successful long-term weight loss fuels the investigation to improve our understanding of the problem of obesity, and effective interventions.

Building upon previous research on the fundamental learning mechanisms that have found to play an important role in overeating and obesity, the aim of the present dissertation was to translate these basic findings to an intervention: food cue exposure therapy. Specifically, previous research has shown that overeating often occurs in response to exposure to food-associated cues, such as the sight, taste and smell of food. Based on the principles of classical conditioning, it is assumed that exposure to food-associated cues can elicit a range of learned responses (i.e., cue reactivity), such as eating desires. Based on the assumption that overeating behaviour is learned, the aim of food cue exposure therapy is to achieve extinction of such associations by exposure to food-related cues without actual food consumption. In practice, food cue exposure therapy entails that participants are instructed to hold, smell and lick the food (i.e., cues), while food intake is not allowed. In the present dissertation, it is investigated whether food cue exposure therapy can be used as a weight loss intervention, by investigating the effects and potential working mechanisms of cue exposure therapy.

The results of this dissertation show that cue exposure therapy is indeed a valuable intervention to incorporate in weight loss interventions for obesity. Regarding the effects of cue exposure, it was found that food cue exposure leads to reduced overeating. Specifically, it was found that food cue exposure leads to less overeating of foods that were included 
during cue exposure, but that these effects do not generalize to foods not included during cue exposure therapy. In addition, food cue exposure therapy leads to reductions of binge eating, and also to weight loss, compared to an active weight-loss intervention. Although further research is necessary on the long-term effects of food cue exposure therapy, this means that cue exposure indeed has high potential to help to improve current weight-loss interventions. In addition, findings of the present dissertation on the potential working mechanisms have yielded important recommendations for the use of food cue exposure therapy in clinical practice (see Products \& Activities).

\section{TARGET GROUPS}

This dissertation has yielded insights into the effects of cue exposure therapy, as well as insights about the working mechanisms of cue exposure therapy that are translated into recommendations for clinical practice. The most important target group that can profit from these insights are clinical psychologists working in mental health care that treat obese clients and patients with Binge Eating Disorder. In addition, other professionals working with obese clients, such as medical doctors, nurses and dieticians also profit from these research findings by having more knowledge about important psychological mechanisms in obesity. Importantly, obese people are also a target group that (indirectly) profit from these findings: creating awareness about psychological mechanisms in obesity and the possibility of tackling these mechanisms in mental health care is of great importance. From a broader point of view, the results of the present dissertation can on the long-term also have great societal relevance when considering the improvement of effective treatments of obesity, and - as a result - the reduction of obesity-associated health costs.

\section{PRODUCTS \& ACTIVITIES}

Based on the results of this dissertation, a number of recommendations have been formulated to improve cue exposure treatments in clinical practice. These recommendations are outlined in Chapter 8, General Discussion: 'Recommendations for clinical practice'.

In order to transfer these insights and clinical recommendations to the main target group, we gave several presentations and workshops for nurses, doctors and therapists of hospitals and other health-care facilities: the paediatric obesity outpatient clinic of Zuyderland hospital, Dutch Obesity Clinic (Nederlandse Obesitas Kliniek; NOK), Center for Obesity Europe (Co-Eur), Area Health Authority (Gemeenschappelijke Gezondheidsdienst; GGD), and U-center. In addition, workshops and presentations were given at Dutch and International conferences for therapists: NIP (Dutch association for student psychologists), VGCt (Dutch association of Behavioural and Cognitive Therapy), NAE (Ducth Academy 
of Eating Disorders) and the EABCT (European Association for Behavioural and Cognitive Therapies). Also, a paper on the effects of cue exposure therapy and recommendations for clinical practice was recently accepted for publication in a Dutch journal for mental health care professionals (De Psycholoog). Further dissemination of knowledge took place via personal consultations of therapists in general mental health care facilities and specialized eating disorder clinics.

Regarding the target group of obese people, the findings of this dissertation have also been communicated via media in women's magazines (Libelle in week 12, 2013 and in week 11, 2016), a popular science magazine (Experiment NL, 2015), national TV (Tros RADAR, 11-11-2013), and the newspaper of the university (Observant, 07-11-2013). Further, several lectures about general principles about the psychology of eating were given to for primary and secondary school children (KidzCollege), and intermediate vocational education students.

We plan to continue knowledge dissemination in the future using the channels described above.

\section{INNOVATION}

The research line of cue exposure therapy as a treatment for overeating and obesity has been introduced quite some time ago. However, the number of studies supporting the beneficial effects of cue exposure therapy for obese individuals was very scarce. In addition, no research was done on the working mechanisms of food cue exposure therapy. The studies in this dissertation are innovative, as these were one of the first to systematically investigate the effects of cue exposure therapy, and the first to investigate working mechanisms of food cue exposure. In addition, the research field of working mechanisms of exposure therapy in anxiety disorders has recently progressed substantially. In this dissertation, we translated these recent insights of exposure therapy in anxiety disorders to for food cue exposure.

In addition to further investigating the (long-term) effects and working mechanisms of cue exposure therapy for obesity in the future, we will also continue this research line of cue exposure therapy with the translation to an important and severely understudied target group: Anorexia Nervosa. Anorexia Nervosa is severe mental disorder, characterized by (extreme) self-starvation and high mortality rates, and appears to be very challenging to treat as many patients do not recover from treatments. Translating the current insights in working mechanisms of cue exposure therapy to exposure therapy for anorexia nervosa is therefore innovative and highly relevant to improve the current treatments of Anorexia Nervosa. 

SAMENVATTING 

De sterke toename in overgewicht en obesitas in de afgelopen decennia hangt samen met de grote overvloed van eten dat overal te koop is. Onze 'obesogene' omgeving zit vol met triggers om te gaan eten, en zet ons zelfs aan tot eten wanneer we geen fysiek hongergevoel hebben. In deze obesogene omgeving zijn het de voedsel-geassocieerde cues die ons aanzetten tot eten, zoals het zien van een snackbar op het station, of de geur van versgebakken wafels in de winkelstraat. Blootstelling aan dergelijke cues zorgt ervoor dat ons lichaam zich alvast gaat voorbereiden op voedselinname, bijvoorbeeld door extra speeksel te produceren en trek te ervaren. Deze verzameling van reacties wordt cue reactiviteit genoemd, en verondersteld wordt dat cue reactiviteit een belangrijke rol speelt bij overeten en gewichtstoename. Leertheoretische modellen suggereren dat cue reactiviteit, in ieder geval voor een belangrijk deel, kan worden aangeleerd door middel van klassieke conditionering. Wanneer bepaalde cues zoals het zien en ruiken van eten (geconditioneerde stimuli; CS) een aantal keren gekoppeld worden met eten (ongeconditioneerde stimulus; US), kan er een cue - voedsel associatie ontstaan. Bij een sterke associatie is enkel de blootstelling aan de cue voldoende om cue reactiviteit aan te wakkeren. Wanneer we ervan uitgaan dat cue reactiviteit een aangeleerde respons is, kunnen deze geconditioneerde reacties ook worden uitgedoofd volgens het principe van extinctie. Tijdens extinctie worden de voedsel-geassocieerde cues (CSen) aangeboden, zonder dat deze worden gevolgd door het consumeren van voedsel (geen US). $\mathrm{Na}$ het verloop van trials neemt de geconditioneerde reactie af. Cue exposure therapie is de klinische vertaling van extinctieleren: de patiënt wordt herhaaldelijk blootgesteld aan de voedsel-geassocieerde cues, zoals het zien, voelen en de ruiken van voedsel, terwijl de consumptie van eten niet is toegestaan. Aan het einde van de sessie wordt het voedsel weggegooid. De enkele bestaande onderzoeken hebben uitgewezen dat cue exposure therapie inderdaad werkt om overeten en eetbuien (i.e., het eten van een ongewone hoeveelheid voedsel binnen een relatief korte tijd, waarbij een gevoel controleverlies wordt ervaren) te verminderen, hoewel deze onderzoeken voornamelijk zijn uitgevoerd bij bulimia nervosa patiënten. Er zijn veel belangrijke openstaande vragen over de effecten van cue exposure, en de werkingsmechanismen van cue exposure zijn dusver nog niet onderzocht. Het doel van dit proefschrift is om de effecten en werkingsmechanismen van food cue exposure therapie te onderzoeken in mensen met overgewicht en obesitas.

Hoofdstuk 1 introduceert food cue exposure therapie als behandeling van overeten en obesitas. De rol van klassieke conditionering bij overeten is beschreven, alsook de vertaling van de principes van extinctie naar cue exposure therapie. Voorgaand onderzoek naar de effecten van cue exposure therapie is beschreven en de potentiele werkingsmechanismen van exposure therapie in het angst domein zijn belicht. Ten slotte zijn de drie hoofdvragen van dit proefschrift beschreven: ten eerste, zorgt cue exposure voor het verminderen van overeten van voedsel dat gebruikt is tijdens cue exposure oefeningen (i.e., blootgestelde voedingsmiddelen), en generaliseren deze effecten van minder overeten ook naar 
voedingsmiddelen die niet tijdens cue exposure aan bod zijn gekomen (niet-blootgestelde voedingsmiddelen)? Ten tweede, kunnen de effecten van cue exposure op het verminderen van overeten verklaard worden door habituatie van cue reactiviteit, en/of door het schenden van verwachtingen over controleverlies? Ten derde, helpt cue exposure therapie om overeten en eetbuien te verminderen, en kan het ook gebruikt worden om gewichtsverlies te bereiken?

De studies in hoofdstukken 2 en $\mathbf{3}$ zijn bijna identiek in experimentele opzet: in beide studies zijn deelnemers met overgewicht en obesitas geïncludeerd (volwassen vrouwen in hoofdstuk 2, jongens en meisjes tussen 12 en 18 jaar in hoofdstuk 3) en volgens het lot toegewezen in een cue exposure interventie (één sessie in hoofdstuk 2 en twee sessies in hoofdstuk 3) of een controle interventie van gelijke lengte gericht op het verbeteren van een gezonde levensstijl en lichaamsbeeld. Proefpersonen ontvingen de interventie in groepen ( 2 tot 7 proefpersonen per groep), terwijl de uitkomst van het onderzoek, namelijk eetgedrag zonder hongergevoel, individueel werd afgenomen. Voedingsmiddelen voor cue exposure sessies werden individueel geselecteerd door de proefpersoon. Voorafgaande aan de interventie gaf de proefpersoon online aan welke voedingsmiddelen het meest lekker én het meest moeilijk waren om vanaf te blijven. De vier meest favoriete voedingsmiddelen werden geselecteerd voor cue exposure. Daarnaast werd één standaard voedingsmiddel toegevoegd aan de cue exposure oefeningen, namelijk chocolade mousse. Tijdens de cue exposure oefeningen werden proefpersonen geïnstrueerd om de voedingsmiddelen vast te pakken, te ruiken, te likken om de trek zo hoog mogelijk op te voeren. Gedurende de cue exposure en de controle interventie werden regelmatig metingen van trek en speekselproductie afgenomen om habituatie van cue reactiviteit binnen de sessies en tussen de sessies vast te stellen. Vóór en na afloop van de interventies werden ook verwachtingen over controle verlies gemeten, om de schending van verwachtingen gedurende therapie vast te stellen. $\mathrm{Na}$ afloop van de interventie, in een staat van verzadiging, nam iedere proefpersoon deel aan een 'smaaktest'. In deze smaaktest werden zes smakelijke toetjes aangeboden aan de proefpersoon, waarbij de proefpersoon werd geïnstrueerd om de smaak van deze toetjes te beoordelen, terwijl in feite enkel de geconsumeerde kilocalorieën werden gemeten. Eén van deze toetjes was ook geïncludeerd tijdens de cue exposure oefeningen (chocolade mousse, het blootgestelde voedingsmiddel) en de overige 5 toetjes waren niet tijdens cue exposure aan bod waren gekomen (niet-blootgestelde voedingsmiddelen). De resultaten van beide studies toonden aan dat proefpersonen in de cue exposure conditie significant minder kilocalorieën hadden gegeten van het blootgestelde voedingsmiddel dan proefpersonen in de controle conditie. Generalisatie van dit gereduceerde inname effect naar niet-blootgesteld voedsel werd niet gevonden in hoofdstuk 2, maar wel in hoofdstuk 3: in dit onderzoek aten proefpersonen ook minder kilocalorieën van niet de niet-blootgestelde voedingsmiddelen in de cue exposure versus controle conditie. In beide studies was habituatie van trek niet geassocieerd met 
inname van kilocalorieën, terwijl lagere verwachtingen over controleverlies na afloop van therapie wel gerelateerd waren aan minder kilocalorie inname in hoofdstuk 2, maar niet in hoofdstuk 3.

In hoofdstuk 4 worden technieken beschreven om inhibitorisch leren te versterken in food cue exposure therapie. Daarnaast wordt in dit hoofdstuk de opzet van een studie beschreven om de effecten van acht sessie cue exposure therapie op trek, verwachtingen over controleverlies, eetgedrag en gewichtsverlies te onderzoeken bij een obese populatie. In deze studie werd de cue exposure interventie vergeleken met een uitgebreide interventie gericht op een gezonde levensstijl. Deze vergelijking vond direct na afloop van de interventies plaats, alsook na drie maanden follow-up. De resultaten van dit onderzoek zijn beschreven in hoofdstuk 5. De cue exposure sessies werden expliciet gericht op het schenden van verwachtingen over controleverlies in plaats van het richten op het habitueren van trek. Voorafgaande aan therapie ondergingen alle deelnemers een uitgebreid interview waarin de individuele favoriete voedingsmiddelen geïdentificeerd werden die betrokken waren bij overeten en/of eetbuien (USen), waarbij ook de voedingscues geïdentificeerd werden (CSen). Ook werden specifieke CS-US verwachtingen uitgevraagd. De bevindingen van dit interview werden via een verslag gerapporteerd aan de therapeut, en werden vervolgens meegenomen om de cue exposure oefeningen tijdens de sessies en huiswerkopdrachten vorm te geven. Om 'deepened extinction' te bereiken werden verscheidene voedsel cues gecombineerd tijdens cue exposure nadat cue exposure werd uitgevoerd met minimaal één van deze cues, zoals het toevoegen van een specifieke televisie serie, tijdstip en gemoedstoestand (CSen) aan de exposure oefening van het bekijken en ruiken van voedsel (CSen). Cue exposure sessies werden uitgevoerd in meerdere relevante contexten, waarbij zorgvuldig rekening werd gehouden met voldoende variabiliteit van de lengte van exposure sessies en honger niveaus. Ook werden occasionele bekrachtigingen tijdens exposure ingevoerd door kleine hapjes te nemen, werden veiligheidssignalen zo veel mogelijk buitengesloten door therapeuten de sessie tijdelijk te laten verlaten en huiswerkopdrachten te gebruiken, en werden geheugensteuntjes ('mental retrieval cues') in het protocol betrokken waarbij de deelnemers actief moesten herhalen wat de belangrijkste leerervaringen tijdens therapie waren. De mate van habituatie van trek gedurende cue exposure (in de cue exposure conditie) en de schending van verwachtingen na afloop van therapie (in beide condities) werden vastgesteld om deze beide maten te kunnen relateren aan behandeleffecten.

In hoofdstuk 5 zijn de resultaten weergegeven van de studie beschreven in hoofdstuk 4. De resultaten van het onderzoek toonden aan dat proefpersonen in de cue exposure conditie significant meer gewicht waren verloren op de nameting en de follow-up meting dan proefpersonen in de controle conditie. Daarnaast was eetbui frequentie marginaal sterker gereduceerd in de cue exposure conditie dan in de controle conditie op de nameting, en significant sterker gereduceerd op 3 maanden follow-up. Wat betreft eetgedrag lieten 
de resultaten zien dat proefpersonen in de cue exposure conditie significant minder kilocalorieën hadden gegeten van het blootgestelde voedingsmiddel vergeleken met de controle conditie, en een trend was gevonden voor minder kilocalorieën inname op followup. Echter, geen verschillen waren gevonden tussen condities op kilocalorie inname van een niet-blootgesteld voedingsmiddel of een algemene (niet-gepersonaliseerde) snack die ook tijdens cue exposure betrokken was. Wat betreft de werkingsmechanismen werd gevonden dat de cue exposure conditie een sterkere schending van verwachtingen had doorgemaakt dan de controle conditie, en dat deze schending van verwachtingen een significante (volledige) mediator was voor verminderde kilocalorie inname van blootgestelde voedingsmiddelen. Echter, schending van verwachtingen speelde geen mediërende rol bij gewichtsverlies of de reductie van eetbuien. Een sterkere habituatie van trek was niet geassocieerd met een betere therapie uitkomst.

In het experiment beschreven in hoofdstuk $\mathbf{6}$ werd onderzocht of het expliciet richten van cue exposure sessies op het schenden van verwachtingen over controleverlies effectiever was dan het richten van cue exposure sessies op het habitueren van trek. Obese vrouwen werden gerandomiseerd in ofwel de cue exposure conditie gericht op het schenden van verwachtingen, ofwel de cue exposure conditie gericht op habituatie, ofwel een controle conditie zonder therapie. Proefpersonen in beide exposure condities ontvingen twee sessies cue exposure therapie. $\mathrm{Na}$ afloop van de therapie werden de geconsumeerde kilocalorieën gemeten - in een staat van verzadiging - van blootgestelde en niet-blootgestelde voedingsmiddelen. Uit de resultaten bleek dat beide exposure condities minder kilocalorieën hadden geconsumeerd van blootgestelde voedingsmiddelen dan de controle groep, terwijl geen verschillen werden gevonden in kilocalorieën tussen beide exposure condities. Wat betreft niet-blootgestelde voedingsmiddelen werden geen verschillen gevonden tussen de drie condities. Bovendien was de lengte van de cue exposure sessies niet significant verschillend tussen beide condities, wat suggereert dat het richten van cue exposure op het schenden van verwachtingen niet efficiënter is dan het richten van cue exposure op habituatie. De mate van habituatie van trek en schending van verwachtingen werden daarnaast ook gemeten gedurende de exposure sessies, en werden ook vooraf en na afloop van therapie gemeten. Schending van verwachtingen bleek gedurende de therapie sessies sterker te zijn in de exposure conditie die expliciet gericht was op het schenden van verwachtingen, terwijl habituatie van trek even sterk plaatsvond in beide exposure condities. Echter, ondanks dat de schending van verwachtingen sterker was in de conditie gericht op het schenden van verwachtingen gedurende de exposure sessies, was de reductie van deze verwachtingen na afloop van therapie even sterk in beide exposure condities. Noch de schending van verwachtingen, noch de habituatie van trek waren significant geassocieerd met de gegeten kilocalorieën van blootgestelde voedingsmiddelen, hoewel lagere verwachtingen na afloop van therapie wel een significante mediator waren tussen 
conditie en de gegeten kilocalorieën van niet-blootgestelde, maar niet van blootgestelde, voedingsmiddelen.

In hoofdstuk 7 werden de data van alle vier studies (hoofdstukken 2, 3, 5 en 6) samengevoegd in één grote dataset om power te vergroten. Met deze grotere dataset werd onderzocht of de effecten van cue exposure op minder inname van blootgestelde voedingsmiddelen wél generaliseerde naar niet-blootgestelde voedingsmiddelen. Daarnaast werden de associaties tussen habituatie van cue reactiviteit, schending van verwachtingen en kilocalorie inname onderzocht. Uit de resultaten van alle studies samen bleek dat cue exposure significant overeten reduceert van voedingsmiddelen die aan bod zijn gekomen tijdens de cue exposure oefeningen, maar dat cue exposure niet leidt tot verminderd overeten van niet-blootgestelde voedingsmiddelen. Sterkere habituatie van trek, gedurende en tussen exposure sessies, was niet gerelateerd aan minder overeten. In tegenstelling tot habituatie waren lagere verwachtingen over controleverlies na afloop van cue exposure en een sterkere reductie gedurende cue exposure wél geassocieerd met minder overeten na cue exposure.

Ten slotte, in hoofdstuk 8, worden de bevindingen betreffende de hoofdvragen samengevat en bediscussieerd, en worden methodologische beperkingen en overwegingen voor toekomstig onderzoek besproken. Enkele aanbevelingen worden genoemd voor cue exposure therapie in de klinische praktijk, en conclusies worden gemaakt betreffende de drie centrale hoofdvragen in dit proefschrift. In het kort, geconcludeerd kan worden dat cue exposure overeten kan verminderen van voedingsmiddelen die tijdens cue exposure oefeningen aan bod zijn gekomen, en dat cue exposure effecten niet generaliseren naar niet-blootgestelde voedingsmiddelen (1). Daarnaast is een sterkere schending van verwachtingen geassocieerd met minder overeten, terwijl een sterkere habituatie van trek niet geassocieerd is met minder overeten (2). En, ten slotte, cue exposure therapie helpt om overeten en eetbuien te reduceren, en om gewichtsverlies te bereiken (3). 


Anita. Er zijn zoveel dingen waarvoor ik je wil bedanken. Allereerst dat je mij hebt uitgekozen om dit mooie project te verwezenlijken, een onderzoekslijn die jij lang geleden bent gestart en waarbij ik de eer heb gekregen om deze voort te zetten. Bedankt voor alles wat je me hebt geleerd, voor de wekelijkse afspraken en de gedetailleerde feedback op mijn stukken, ondanks jouw vaak onmogelijk drukke agenda. Bedankt ook dat je me mee nam naar het lab van Kerri Boutelle in San Diego, en het lab van Michelle Craske in Los Angeles. Maar nog de meeste dank voor jouw vertrouwen in mij, en de geruststelling dat het altijd wel goed zou komen met de projecten, ondanks alle omstandigheden. Je bent zeer betrokken en hebt ervoor gezorgd dat ik de afgelopen jaren het meeste uit mezelf heb gehaald. Veel dank, voor alles.

Anne. Ook jou wil ik bijzonder bedanken voor al je hulp in de afgelopen jaren. Als copromotor heb jij ook een zeer belangrijke rol gespeeld in mijn promotie. Ik kon altijd bij jou terecht met de meest uiteenlopende vragen en je had altijd tijd voor me, no matter what. Ik heb veel van je geleerd, zowel op professioneel gebied als de balans tussen werk en privé. Anita en Anne, ik heb bewondering voor jullie werkattitude. In deze moeilijke omgeving om als vrouw naar de top te komen is dit voor jullie beiden gelukt. Ik ben trots dat ik mijn promotie onder jullie begeleiding heb kunnen vervullen.

Eigenlijk hoort Karolien ook een beetje bij mijn begeleiders. Sinds het opzetten van de RCT en het begeleiden van 8 master studenten in dit project zijn wij min of meer onafscheidelijk geworden op het werk. We hebben samen talloze presentaties gegeven. Daarnaast ben jij ook een vriendin geworden met wie ik heel hard kan lachen, zowel op het werk (/op onze kamer) als daarbuiten tijdens zoals congressen, etentjes, EPP en de memorabele Summer School in Leuven. Ik vond het top dat ik jouw paranimf mocht zijn tijdens jouw verdediging, en vind het zeer fijn dat je ook mij in de laatste fase heb ondersteund, dankjewel.

Graag wil ik ook de leden van mijn beoordelingscommissie bedanken voor hun belangrijke bijdrage aan mijn promotie: Prof. Dr. Johan W. S. Vlaeyen, Dr. Chantal Nederkoorn, Prof. Dr. Marleen M. Rijkeboer, Prof. Dr. Marcel A. Van den Hout, en Dr. Bram Vervliet.

Jantine. Ik prijs me gelukkig dat we sinds de Research Master zo'n hecht bevriend zijn geraakt en sindsdien een exact parallelle carrière hebben kunnen doorlopen. We hebben elkaars hoogte- en dieptepunten meegemaakt, en je kent mij door en door. Als echte optimist weet jij de tegenslagen in een juist perspectief te plaatsen, waardoor ik er net wat beter mee kan dealen. Ik vond het super dat ik jouw paranimf mocht zijn tijdens jouw verdediging, en ik ben dankbaar dat jij ook mijn paranimf wilt zijn en me ondersteund hebt op weg naar mijn $\mathrm{PhD}$.

Lotte. Jouw aanstekelijke positieve levensinstelling helpt me om vaak nog even extra gas te geven voor een naderende deadline. 32 spierballen. Helemaal bijzonder was de verassing 
dat je voor mijn deur stond op de dag dat dit proefschrift naar de drukker moest, en dat je me hielp met al die referenties! Ik kan je hier niet genoeg voor bedanken. Ook een speciale dank aan Josefien en Tiny, die me zo goed hebben geholpen om af te schakelen van werk. Je hebt een geweldige humor en ik hoop dat we samen nog heel wat bitterbal-workshops mogen geven.

Lorraine. Mijn ren- en pumpmaatje waarbij we samen alle frustraties eruit hebben gesport. Eerst de laatste loodjes van mijn proefschrift en daarna die van jou. Dankjewel voor je luisterend oor en ik hoop dat we nog lang samen onze spierballen zullen trainen.

Jessica. Dank aan mijn kamergenoot voor het grootste gedeelte van mijn promotie. Je was vaak betrokken bij mijn afwegingen en dilemma's, en had altijd goed advies. We hebben ook veel gelachen en spelletjes gespeeld, samen met je echtgenoot Kai. Verder ook een dankwoord aan alle andere (oud) kamergenoten: Conny, Linda, Alfons, Sarah, Bart en Hanne.

De eetgroep: Anita, Anne, Sjaan, Sandra, Katrijn, Carolien, Remco, Karolien, Fania, Iris, Sieske, Lotte, Jessica, Eric, Valerie, Bart, Sarah, Leonardo, Yu, en oud-leden Peggy, Bastiaan, Nele, Jessica W., Astrid, Janneke en Harris. Ik heb altijd genoten van de eat meets en brain days, waarin we als groep nadachten over nieuwe onderzoekideeën en designs. Jullie zorgden ervoor dat ik mij thuis voelde in deze warme en stimulerende groep, en hebben me veel geholpen in het optimaliseren van designs en interpreteren van mijn data. Een speciale dank ook aan de leden van de Writing Club: Fania, Iris, Sieske, Thomas, Kai, Lotte en Jessica. Bedankt voor de steun vlak voor mijn deadlines, de no-nonsense 25 -minuten timer die me een perfecte focus bracht, en de gezellige pauzes. Dankjulliewel.

Lindy, Caroline, Marionne, Truus, en Jessie in het bijzonder: ik heb veel beroep op jullie gedaan, en ben dankbaar voor de altijd supersnelle praktische hulp. Ook een speciale dank aan Marionne voor de praktische voorbereidingen van de eindeloze VAS schalen en speekselzakjes voor mijn onderzoeken.

Jill en Marisol. Jullie hebben mij als student geïnspireerd om onderzoeker te worden. Dank dat jullie mij hebben geholpen bij de belangrijke keuzes tijdens mijn studie.

Uiteraard een speciale dank aan alle deelnemers aan mijn onderzoeken. Ook veel dank aan kinderarts Barbara Vernooy van het Zuyderland ziekenhuis in Kerkrade en de verpleegkundigen van de GGD Zuid Limburg die mij hielpen om deelnemers te mogen benaderen. Dank aan Jacques van Lankveld om ruimtes ter beschikking te stellen bij de Open Universiteit Heerlen om mijn onderzoek uit te kunnen voeren. Daarnaast ook dank aan de stagiaires die hebben bijgedragen aan de dataverzameling: Jette, Anastacia, Marcella, Caroline, Rosanne, Dominique, Jeannette, Evalien, Denise, Sarah en Rianne. 
Daarnaast wil ik nog enkele vrienden in het bijzonder bedanken voor de nodige afleiding en interesse in mijn promotie. Maud/Frans, Imke en Farah, voor jullie lieve berichtjes en lekkere etentjes om af te schakelen van het werk, en de mooie gebeurtenissen die we samen hebben mogen delen. Koen \& Metha, voor de gezellige biertjes en wijntjes die we samen gedronken hebben, de speciale band die is ontstaat nu we praktisch buren zijn geworden, en natuurlijk het hosten van mijn promotiefeest. Werner \& Lara, voor de weekendjes samen met Bruno \& Puk met alle wijntjes, dance-offs en ongelimiteerde gezelligheid; WMD. Kars, schoonbroer, voor je positieve feedback na iédere hele alinea die ik in mijn proefschrift heb geschreven, de vele cava’s en één enkele prosecco.

Mijn lieve familie, die door dik en dun voor me hebben klaargestaan. Alfons en Marjo, jullie zijn mij zo dierbaar, niet in het minste voor de kleine dingen zoals hulp bij de was of het meegegeven van een bord eten in tijden dat ik niet achter mijn laptop was weg te slaan. Heel erg bedankt voor jullie onvoorwaardelijke steun en warmte. Piet(ie), de stille kracht die altijd voor mij klaarstaat. Het is fijn om bij jou te zijn met jouw altijd positieve insteek in het leven, en de lekkere wafels. Sandra en Armand, bedankt voor jullie eindeloze energie en enthousiasme om met de familie gezellig aan tafel te zitten. Dank aan mijn broertjes Alex, René en Harald, en natuurlijk Ellen, Arlette en Constance, voor de gezellige gesprekken en goede adviezen. En natuurlijk een dikke knuffel aan mijn petekindjes Kim en Lincy. Veel dank ook aan Raoul en Josien voor het feit dat jullie er altijd zijn. Xandra, voor de leuke gesprekken over onderzoek en ontwikkeling, maar ook daarnaast een voorbeeld te zijn voor een perfecte work-life balance. Betty en Ernst, voor de ongelimiteerde interesse in mijn onderzoek en keuzes en de zeer goede adviezen. Josje, voor de warme gesprekken over het werk en de hectiek van het leven. En oma, bedankt voor je liefdevolle betrokkenheid in mijn (werk) leven, en bedankt voor de goede gesprekken, al dan niet tijdens de witte port met (veel) ijs.

Vanuit hoog in de lucht kijken twee belangrijke mensen mee. Mam, Alice, je hebt mij van baby af aan meegemaakt in alle fases van mijn leven, tot bijna het einde van mijn promotie-periode. Ik koester de wijze levenslessen die je me mee hebt gegeven; ik mis je zo. Mama, jij bent mijn grote voorbeeld geweest om mijn huidige (werk)leven in te richten. Jij hebt mij geïnspireerd om te gaan schrijven (al ben ik niet half zo goed) en we hebben tijdens onze wandelingen zo veel belangrijke keuzes gemaakt waar ik nog iedere dag dankbaar naar terugkijk. Het doet nog zo'n zeer dat je er niet meer bent, en dat je het einde van mijn promotie niet hebt kunnen meemaken, maar ik ben dankbaar voor alles dat je me hebt meegegeven. Dankzij jou haal ik alles uit mezelf, en ben ik gevormd tot de vrouw die ik nu ben. Lites finiri oportet.

Papa. Hard werken en nooit opgeven heb ik ook ongetwijfeld van jou geërfd. Je leert me dat een doel altijd te behalen is. Je wees me als 12-jarige met teleurstellend studieadvies op Einstein's quote over genialiteit: $1 \%$ inspiratie, $99 \%$ transpiratie. Dit is een van mijn 
levensmotto's geworden. Naast hard werken heb je me ook geleerd om te genieten van het leven: tijdens vakanties, maar ook met een Belgisch speciaal biertje en de hondjes in de tuin. Ik bewonder jouw enorme veerkracht. Jij hebt altijd goede zin en relativeert mijn zorgen. Je leert me het beste te zien in anderen (bijzonder aardig), en in mijzelf. Dankjewel.

Florence. Mijn liefste susje. Jij weet als geen ander hoe ik functioneer, en geeft me een zet in de juiste richting als ik dat nodig heb. Je bent zeer waardevol geweest in mijn ontwikkeling in de afgelopen jaren, en mijn grote steun om met alle verdrietige gebeurtenissen om te gaan. Daarnaast helpt jouw ongelofelijke humor om iedere dag weer leuk te maken. And if I fly, or if I fall, least I can say I gave it all. We zetten tradities voort, en vieren de mooie momenten: groot en klein. Now sissy that walk!

Rémon. Tijdens mijn promotie zijn we op stel en sprong getrouwd en hebben we ons huis gekocht dat we bijna volledig hebben gesloopt en weer helemaal terug hebben opgebouwd. Veel mensen hebben ons geholpen bij dit enorme project, maar alles verliep onder jouw strikte regie. Bijna iedere schroef, balk, kabel en dakpan heb jij in jouw handen gehad, en dit alles zonder stress. Ik heb grote bewondering voor je eindeloze energie en de manier hoe jij de meest onmogelijke projecten tot een goed einde kunt brengen. Je hielp me om mijn zorgen om mijn proefschrift te relativeren. Samen met jou heb ik de verdrietige gebeurtenissen een plek kunnen geven. Je brengt rust in mijn leven. Met jou kan ik alles aan. Alles. Ik hou zielsveel van je. 




\section{CURRICULUM VITAE}



Ghislaine Léonie Thérèse Schyns was born on the $19^{\text {th }}$ of February 1988 in Maastricht, the Netherlands. In 2005, she graduated from secondary school (HAVO, Sophianum, Gulpen) after which she studied Food and Dietetics for one year in 2006 (HBO propaedeutic year; Hogeschool of Arnhem and Nijmegen, Nijmegen), in order the get accepted for her bachelor studies at Maastricht University. In 2009, Ghislaine received her bachelor degree at the faculty of Health, Medicine and Life Sciences, specialization in Mental Health, at Maastricht University. She subsequently followed the Master Mental Health at the faculty of Health, Medicine and Life Sciences, during which she did a clinical and research internship at the Memory Clinic, Maastricht University Medical Center (MUMC+). In 2010, she received her Master's degree, and received a Top 3\% award. She ten completed a second master: a two-year Research Master in Cognitive and Clinical Neuroscience at the Faculty of Psychology and Neuroscience (specialization Psychopathology), and graduated from this Master in 2012 (Cum Laude). During the research master, Ghislaine did two research internships at the Eating Disorders and Obesity group at the Department of Clinical Psychological Science, Maastricht University. During her studies, she also worked as a research assistant in various research projects at this department.

Ghislaine started working as a PhD candidate in 2012 in the Eating Disorders and Obesity group at the Department of Clinical Psychological Science, Maastricht University, under the supervision of Prof. Dr. Anita Jansen and Dr. Anne Roefs. During her PhD, she obtained her University Teaching Qualification. Since November 2017, Ghislaine works as an Assistant Professor Tenure Track, also in the Eating Disorders and Obesity group at the Department of Clinical Psychological Science, Maastricht University. 


\section{PUBLICATIONS}

Schyns, G. (in press). Ruiken tot je een ons (minder) weegt. De Psycholoog.

Schyns, G., van den Akker, K., Roefs, A., Hilberath, R., \& Jansen, A. (2018). What works better? Food cue exposure aiming at the habituation of eating desires or food cue exposure aiming at the violation of overeating expectancies? Behaviour Research and Therapy, 102, 1-7. https://doi.org/10.1016/j.brat.2017.12.001

Schyns, G., van den Akker, K., Roefs, A., Houben, K., \& Jansen, A. Weight loss and binge eating reduction after eight sessions food cue exposure therapy. In revision at Journal of Consulting and Clinical Psychology.

Schyns, G., Roefs, A., Smulders, F. T. Y., \& Jansen, A. (2018). Cue exposure therapy reduces overeating of exposed and non-exposed foods in obese adolescents. Journal of Behavior Therapy and Experimental Psychiatry, 58, 68-77. https://doi.org/10.1016/j. jbtep.2017.09.002

van den Akker, K., Schyns, G., \& Jansen, A. (2017). Altered appetitive conditioning in overweight and obese women. Behaviour Research and Therapy, 99, 78-88. https://doi. org/10.1016/j.brat.2017.09.006

Jansen, A., Schyns, G., Bongers, P., \& van den Akker, K. (2016). From lab to clinic: Extinction of cued cravings to reduce overeating. Physiology and Behavior, 162, 174180. https://doi.org/10.1016/j.physbeh.2016.03.018

Schyns, G., Roefs, A., Mulkens, S., \& Jansen, A. (2016). Expectancy violation, reduction of food cue reactivity and less eating in the absence of hunger after one food cue exposure session for overweight and obese women. Behaviour Research and Therapy, 76, 57-64. https://doi.org/10.1016/j.brat.2015.11.007

Schyns, G.,van den Akker, K., \& Jansen, A. (2016). Enhancing inhibitory learning to reduce overeating: Design and rationale of a cue exposure therapy trial in overweight and obese women. Contemporary Clinical Trials, 49, 85-91. https://doi.org/10.1016/j. cct.2016.06.008

Vervoort, L., van den Akker, K., Schyns, G., Kakoschke, N., Kemps, E., \& Braet, C. (2016). Automatic processes in eating behavior: understanding and overcoming food cuereactivity. In R. G. Menzies, M. Kyrios, \& N. Kazantzis (Eds.), Innovations and future directions in the behavioural and cognitive therapies (pp. 1-2).

Werthmann, J., Jansen, A., Vreugdenhil, A. C. E., Nederkoorn, C., Schyns, G., \& Roefs, A. (2015). Food Through the Child's Eye: An Eye-Tracking Study on Attentional Bias for Food in Healthy-Weight Children and Children With Obesity. Health Psychology, 34(12), 1123-1132. https://doi.org/10.1037/hea0000225

Alleva, J., Schyns, G., \& Martijn, C. (2014). Positief lichaamsbeeld: Wat betekent het werken aan een positief lichaamsbeeld voor de therapeut en de cliënt? Directieve Therapie, 34(4), 250-256. 


\section{PRESENTATIONS}

Schyns, G., Lemmens, L. (2017). Can't stop the feeling? Workshop cue exposure therapie voor het verminderen van overeten bij eetstoornissen en obesitas. Workshop at the annual conference of the Association of Behavioural and Cognitive Therapy (VGCt), Veldhoven, the Netherlands.

Schyns, G., van den Akker, K., Roefs, A., Hilberath, R., \& Jansen, A. (2017). Schenden van verwachtingen in cue exposure therapie voor obese vrouwen. Oral presentation at the annual conference of the Association of Behavioural and Cognitive Therapy (VGCt), Veldhoven, the Netherlands.

van den Akker, K., Schyns, G. (2017). Invited psychology \& eating workshop for honours program intermediate vocational education students, Gilde Opleidingen, Venlo, The Netherlands.

Schyns, G., Roefs, A., Jansen, A. Cue exposure therapy to reduce overeating. Learning not to eat. Oral presentation at the Research Day of the Faculty of Psychology and Neuroscience, Maastricht University, Rijckholt, The Netherlands.

Schyns, G., Roefs, A., Jansen, A. Cue exposure therapie voor het verminderen van overeten: de rol van habituatie (2016). Oral presentation at the annual conference of the Association of Behavioural and Cognitive Therapy (VGCt), Veldhoven, the Netherlands.

Schyns, G., Roefs, A., Jansen, A. Workshop cue exposure therapy om overeten te reduceren. Leren om niet te eten (2016). Annual day of NIP student psychologists, Eindhoven, the Netherlands.

Schyns, G., Roefs, A., Jansen, A. (2016). Oral presentation 'experience your future' for bachelor students of Wageningen University, Maastricht, the Netherlands.

Schyns, G., van den Akker, K. Lecture for 'Kidzcollege' (2016): Oral presentation and demonstration on general principles of psychology in primary school children, Maastricht, The Netherlands.

Schyns, G., Roefs, A., Jansen, A. (2016). Food cue exposure therapy to reduce overeating: the role of habituation. Poster presentation at the Research Day of the Faculty of Psychology and Neuroscience, Maastricht University, Maastricht, The Netherlands.

Schyns, G., Roefs, A., Jansen, A. (2016) Cue exposure therapy to reduce overeating: learning not to eat. Oral presentation at the symposium 'conditioning and psychopathology, theoretical advances and clinical applications' of the Dutch-Flemish postgraduate school of Experimental Psychopathology (EPP). Heeze, The Netherlands.

Schyns, G., Roefs, A., Jansen, A. (2016). Cue exposure therapy for overeating. Oral presentation during Battle of the Brains at the EPP research day, Utrecht, The Netherlands. Winner of EPP Battle of the Brains.

Schyns, G., Roefs, A., Jansen, A. (2015). Exposure-nieuwe-stijl voor mensen met obesitas: hoe werkt het? Oral presentation at U-Center, Epen, The Netherlands. 
Schyns, G., van den Akker, K., Jansen, A. Exposure-nieuwe-stijl voor mensen met obesitas: werkt het? (2015). Oral presentation at the annual conference of the Association of Behavioural and Cognitive Therapy (VGCt), Veldhoven, the Netherlands.

Schyns, G., Roefs, A., Jansen, A. (2015). Conquer your craving: Why cue exposure helps not to eat. Oral presentation at the Research Day of the Faculty of Psychology and Neuroscience, Maastricht University, Rijckholt, The Netherlands.

Schyns, G., Roefs, A., Mulkens, S. Jansen, A. Expectancy violation, reduction of food cue reactivity and less eating in the absence of hunger after one food cue exposure session for overweight women (2015). Poster presentation at the summer school on Emotional Learning and Memory in Health and Psychopathology at KU Leuven, Leuven, Belgium.

Schyns, G., van den Akker, K., Dassen. F. Lecture for 'Kidzcollege' (2015): Oral presentation and demonstration on general principles of psychology in primary school children, Maastricht, The Netherlands.

Schyns, G., Roefs, A., Mulkens, S. Jansen, A. Expectancy violation, reduction of food cue reactivity and less eating in the absence of hunger after one food cue exposure session for overweight women (2014). Poster presentation at the conference day of the Dutch Academy of Eating Disorders (NAE), Zwolle, The Netherlands. Winner of conference's poster price.

Schyns, G., Roefs, A., Mulkens, S. Jansen, A. Expectancy violation, reduction of food cue reactivity and less eating in the absence of hunger after one food cue exposure session for overweight women (2014). Oral presentation at the European Association for Behavioural and Cognitive Therapies (EABCT), Den Haag, The Netherlands.

Schyns, G., Roefs, A., Mulkens, S. Jansen, A. Expectancy violation, reduction of food cue reactivity and less eating in the absence of hunger after one food cue exposure session for overweight women (2014). Oral presentation at the University California San Diego (UCSD), San Diego, United States of America.

Schyns, G. Workshop on cue exposure (2014). Centre for Obesity Europe (CO-EUR), Vught and Maastricht, The Netherlands.

van den Akker, K. \& Schyns, G. Invited lecture 'experience your future' (2014) for bachelor students of Radboud Universiteit Nijmegen, Nijmegen, The Nederlands.

Schyns, G., Albers, R. Workshop on cue exposure (2014). Workshop at the conference day of the Dutch Academy of Eating Disorders (NAE), Maastricht, The Netherlands

Schyns, G., Jansen, A., Roefs, A. (2014). Conquer your craving. Cue exposure training for morbidly obese adults in pre-operative phase for bariatric surgery. Poster presentation at the Research Day of the Faculty of Psychology and Neuroscience, Maastricht University, Rijckholt, The Netherlands. 
Schyns, G., Jansen, A., Nederkoorn, C., Roefs, A. (2013). Psychological predictors of BMI change in dieters using a web-based CBT coaching program. Oral presentation at the British Feeding and Drinking Group Annual Meeting, Loughborough, United Kingdom.

Schyns, G., Jansen, A., Roefs, A. (2013). Conquer your craving. Cue exposure training for overweight children. Poster presentation at the Research Day of the Faculty of Psychology and Neuroscience, Maastricht University, Rijckholt, The Netherlands.

Schyns, G., van den Akker, K. Lecture for 'Kidzcollege' (2013). Oral presentation and demonstration on general principles of psychology in high school children, Stella Maris College, Meerssen, The Netherlands.

Giesen, J. C. A. H., Schyns, G., Roefs, A., (2012). Tailoring dieting strategies to the 'toxic' environment. The influence of restriction versus daily consumption of chocolate on its reinforcing value. Poster presentation at Endo-Neuro-Psycho Meeting, Lunteren, the Netherlands. 
